Portland State University

PDXScholar

$1-1-2010$

\title{
Identifying Best Quality Management Practices for Achieving Quality and Innovation Performance in the Forest Products Industry
}

Scott A. Leavengood

Portland State University

Follow this and additional works at: https://pdxscholar.library.pdx.edu/open_access_etds Let us know how access to this document benefits you.

Recommended Citation

Leavengood, Scott A., "Identifying Best Quality Management Practices for Achieving Quality and Innovation Performance in the Forest Products Industry" (2010). Dissertations and Theses. Paper 136. https://doi.org/10.15760/etd.136

This Dissertation is brought to you for free and open access. It has been accepted for inclusion in Dissertations and Theses by an authorized administrator of PDXScholar. Please contact us if we can make this document more accessible: pdxscholar@pdx.edu. 
Identifying Best Quality Management Practices for Achieving Quality and Innovation Performance in the Forest Products Industry

by

Scott Allen Leavengood

A dissertation submitted in partial fulfillment of the requirements for the degree of

\author{
Doctor of Philosophy \\ in \\ Systems Science: Engineering Management
}

Dissertation Committee:

Timothy R. Anderson, Chair

Wayne Wakeland

Tugrul Daim

Tom Gillpatrick

Robert D. Dryden

Portland State University

(C)2011 


\begin{abstract}
In many business sectors today, the focus on quality as a competitive tool is being replaced by a focus on innovation. Research exploring connections between quality management, innovation, and company performance suggests that quality is 'necessary but insufficient' in today's business environment. In short, the question facing managers, particularly those in small firms, is how to adapt their quality management practices to achieve innovation performance in addition to quality performance.
\end{abstract}

To answer this question, West Coast U.S. forest products manufacturers were surveyed about quality management practices and performance with respect to both quality and innovation. Quality management practices were assessed following the systems perspective articulated by the Malcolm Baldrige National Quality Award. Data envelopment analysis was used to identify companies efficiently using quality management practices to lead to quality and/or innovation performance. Survey responses from the efficient firms were then analyzed via cluster analysis to identify two categories of firms: those achieving primarily quality outcomes and those achieving both quality and innovation outcomes. Executives from two firms in each category were interviewed to provide detail on the management practices used by the companies. Interview transcripts were examined to identify similarities and differences in practices between the two categories of firms. 
Results suggest several specific areas of focus for firms wanting to adapt their quality management practices to achieve both quality and innovation performance. For example, firms focused on innovation proactively seek to identify and meet customers' needs whereas quality-focused firms primarily emphasize reacting to customer complaints. More specifically with respect to 'customer focus', firms focused on innovation emphasize convenience for their customers through practices such as standardizing product lines and providing product specifications on their websites. In contrast, neither quality-focused firm had a website. These firms were at their production capacity (at least prior to the recession) and viewed websites strictly as a means to attract new business rather than as a service to existing customers. Also with regards to customer focus, firms focused on innovation sought to generate new business - not just for their company, but for their customers as well. Beyond customer focus, firms focused on innovation provide employees with opportunities to help the organization implement changes. With respect to benchmarking, firms focused on innovation actively sought to measure their performance against the "best practice' in the industry; firms focused primarily on quality performance demonstrated little if any emphasis on benchmarking. Finally, there were apparent overarching and hence cultural differences between the two categories of firms - firms focused on innovation were more proactive, strategic, and willing to take risk; in addition, these firms discussed innovation as the means to improve product quality, reduce costs, or attract new customers. By contrast, the quality-focused firms were reactive, conservative, and risk-averse; these firms discussed innovation primarily as 'technology' without reference to potential linkages to company performance. 


\section{Acknowledgements}

To my family - thanks for enduring 9+ years of me being a 'bit preoccupied.' To my beautiful wife of nearly 20 years, thank you so much for hanging in there with me through thick and thin with this! Without your love and support, I would have given up long ago. To my daughters, now 'dad's home' means he's really home (and not sitting in the office on the computer...).

To my mom - thanks for demonstrating for me the value of education.

To my colleagues Eric Hansen and Chris Knowles - thanks for your insights and encouragement.

To members of the Oregon Wood Innovation Center Advisory Council (Roy Anderson, Blake Holton, Lee Jimerson, David Schmidt, and David Stallcop) - thanks for reviewing the interview questions. And to the survey respondents and interviewees - thanks for taking the time to respond to 'yet another survey!'

To Mark Edwards, my friend and colleague - thank you for all your advice and support. And thanks for taking the time to read several drafts of my methods - only a true friend would do that! 
And last but not least, many thanks to my committee, Drs. Anderson, Wakeland, Daim, Dryden, and Gillpatrick. I greatly appreciate your wisdom and guidance - and in particular, your patience enduring my submission of a couple very long drafts right at the deadline! 
Table of Contents

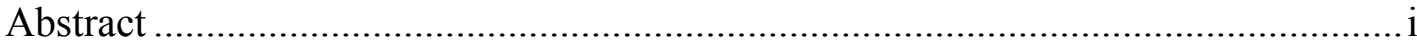

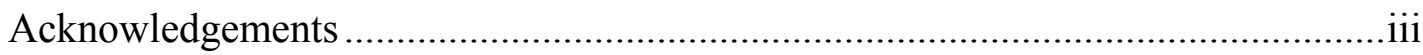

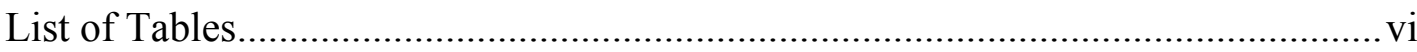

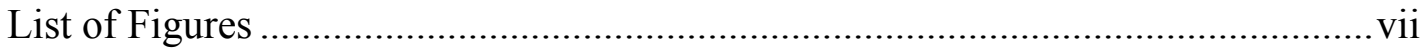

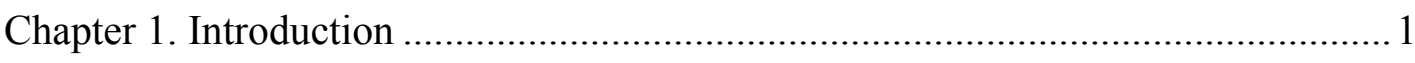

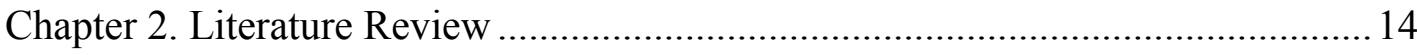

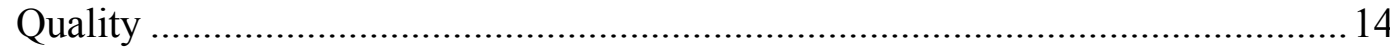

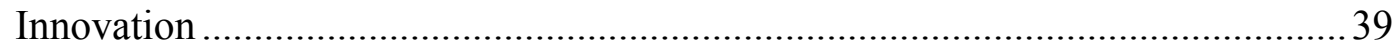

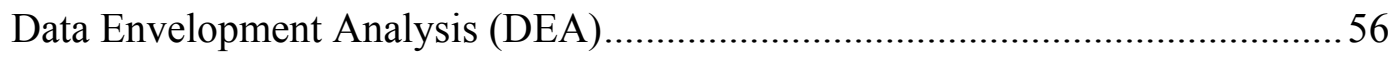

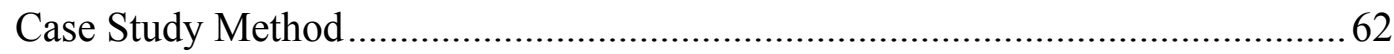

Relationships between Quality Management and Organizational Performance ... 69

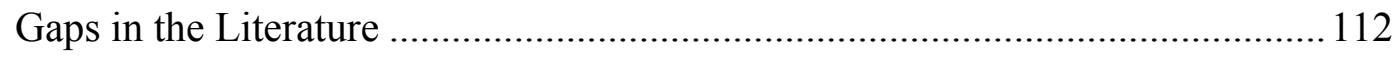

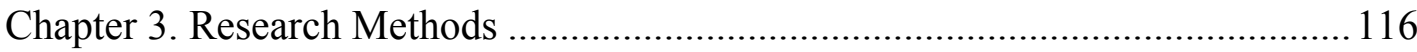

Chapter 4. Results \& Discussion......................................................................... 147

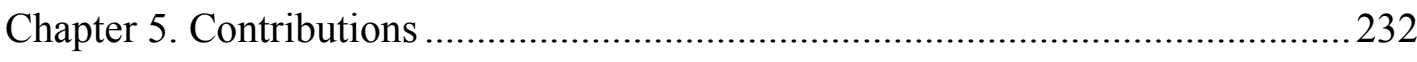

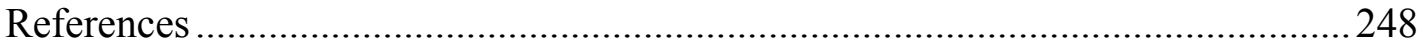

Appendix A - Quality Constructs and Measurement Instruments.......................263

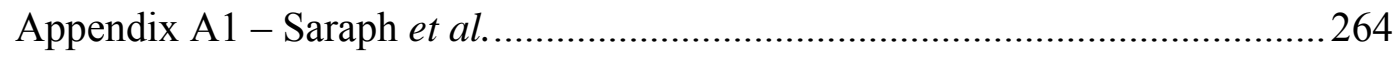

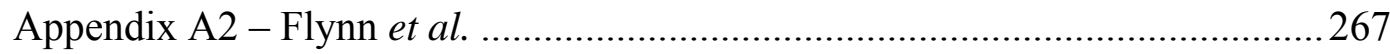

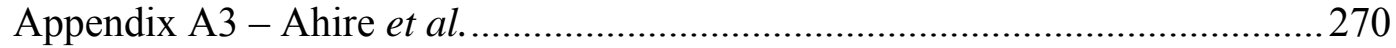

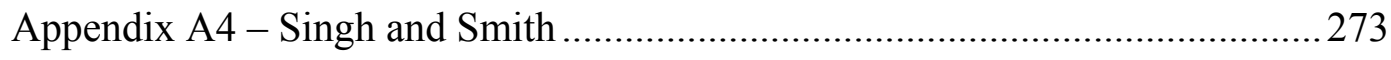

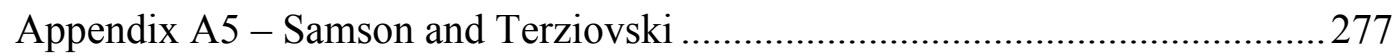

Appendix A6 - Final Measurement Instrument Used ..................................2 280

Appendix A7 - Cover Letter for Questionnaire ...........................................228

Appendix A8 - Case Study Interview Questions ............................................285

Appendix B - Innovation Constructs and Measurement Instruments ...................228

Appendix C - Graphical Representation of Efficiency ...................................... 290

Appendix D - Results of Test for Non-response Bias ......................................2291

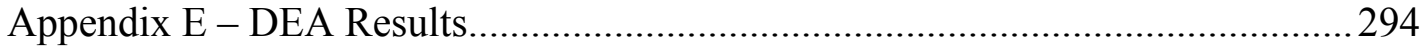




\section{List of Tables}

Table 1. Performance Measures for Each Critical Factor of TQM [71] .......................37

Table 2. Scales to Measure Product and Process Innovation Performance [25] ...........54

Table 3. Distinctive Principles Associated with TQC and TQL Compared to Common

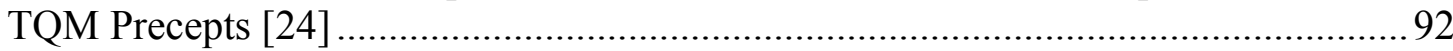

Table 4. Summary of Conflicting Arguments on the Relationship Between TQM and

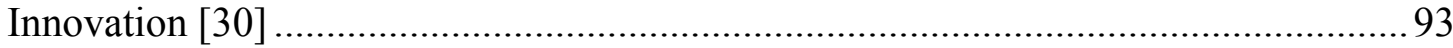

Table 5. Constructs for Measuring Technology and R\&D Management [35] ........... 102

Table 6. Summary of Literature Exploring Relationships between Quality and

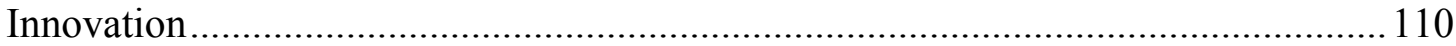

Table 7. Respondents by Industry Sector ................................................................... 150

Table 8. Number of Employees in Population vs. Survey Respondents ...................... 151

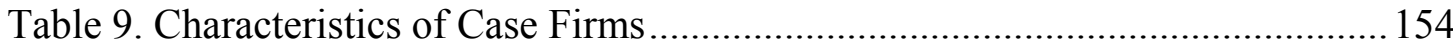

Table 10. Cross-Case Comparison of Questionnaire Responses (Inputs)................... 166

Table 11. Cross-Case Comparison of Questionnaire Responses (Outputs) ................ 166

Table 12. Quality Management Practices that Differ between Balanced and Quality-

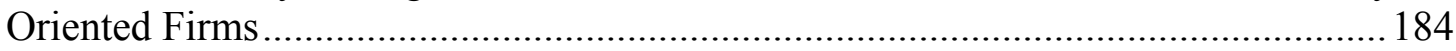

Table 13. Number of Comments Related to Hard and Soft TQM Factors.................. 188

Table 14. Recommended Practices for Proactive Customer Interaction.....................231 


\section{List of Figures}

Figure 1. Baldrige Criteria Framework: A Systems Perspective [61] .......................21

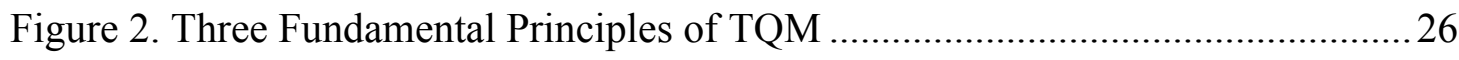

Figure 3. Dualities within TQM Implementation ..............................................2 27

Figure 4. A Multidimensional Model of Innovation [72] ...................................... 42

Figure 5. Innovation and Stage of Development [78] ........................................ 46

Figure 6. TQM and Innovation: Research Framework [30] ................................. 98

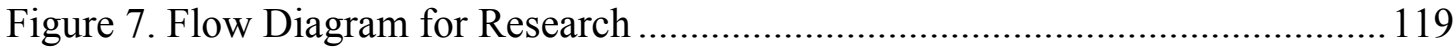

Figure 8. DEA Input-Output Model Used in this Study ....................................... 126

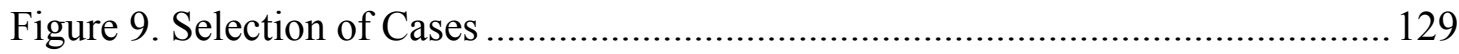

Figure 10. Cluster Analyis Dendrogram and Average Performance Responses for

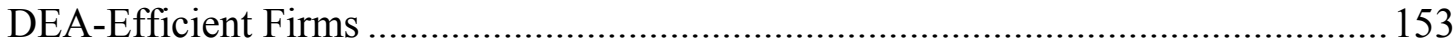




\section{Chapter 1. Introduction}

Organizations have long recognized the importance of quality. Prior to the industrial revolution, quality was ensured through apprenticeship programs, skilled craftsman, and the training and standards of professional guilds. Mass production systems led to a transition to relying on product inspection for quality assurance. Beginning in World War II, the quality sciences and profession grew rapidly. The 'quality movement' began with a focus on specific tools such as acceptance sampling procedures and Statistical Process Control (SPC); efforts were centered primarily in production [1]. Quality management programs eventually grew to encompass a company-wide, i.e., systems-based approach which is now known as Total Quality Management (TQM) [2].

Widely publicized failures of TQM to deliver bottom-line results led many management experts to declare TQM a failure and/ or pose the question, is TQM dead [3-8]? Numerous researchers have studied the impacts of TQM on performance and the results are mixed. Regardless, early approaches to implementing TQM were said to over-emphasize quality-related metrics to the exclusion of financial metrics, i.e., bottom-line results. Further, TQM has been criticized for failing to emphasize 'breakthrough' improvements. That is, TQM, and continuous improvement by extension, are said to focus on evolutionary rather than revolutionary improvement. 
Business Process Reengineering (BPR) and later, Six Sigma, were established to address the perceived weaknesses of TQM. And of course, TQM itself continues to evolve.

BPR emphasizes radical improvement by completely revamping processes, or eliminating unnecessary processes altogether, rather than simply making incremental improvements [9]. Said another way, where the continuous improvement philosophy might lead practitioners to pose the question "how can we do this (process) better?", in BPR one would ask, "do we need this process at all?" BPR faded in popularity in the 1990s due to perceived linkages with downsizing, i.e., employees began to associate reengineering with 'improving the bottom line via layoffs' [10]. Drawing distinctions between BPR and TQM has been a subject of much debate. Some see BPR as a standalone philosophy that is a replacement for TQM. Others have argued that BPR is simply a subset of TQM albeit with a focus on breakthrough vs. incremental improvement where feasible [11, 12].

Six Sigma was also developed in part to address perceived weaknesses of TQM. Six Sigma integrates well-established quality tools and techniques into a structured approach (DMAIC - define, measure, analyze, improve, control) to quality improvement projects and by emphasizing project selection. Projects are selected based on the potential for significant positive impact on profitability. As with BPR, some see Six Sigma as an alternative or replacement for TQM while others argue that 
there is nothing new in Six Sigma; it is simply 'TQM repackaged' [13-15]. The principle of reducing variation to improve quality remains.

In discussing the evolution of TQM and debate over whether competing philosophies are truly new, it should become clear that there is no single universally accepted definition of TQM. A significant amount of research has been devoted purely to defining TQM and developing constructs to differentiate 'TQM firms' from 'non-TQM firms' [16-20].

Further, part of the evolution of TQM has been to recognize the importance of adapting the philosophy to suit the strategy, context, and culture of the firm and thus the need for a 'contingency approach' to implementing TQM. Early TQM programs emphasized the technical or 'hard' tools such as statistical process control, Pareto charts, and design of experiments. Over time, practitioners recognized the inadequate attention being paid to the systems approach fundamental to TQM, primarily the need to address the human side of quality. Such recognition resulted in greater emphasis on the 'soft' factors such as teams and employee empowerment [21].

In fact, researchers have revealed other potential dualities within TQM beyond the 'hard vs. soft' factors [22-24]. One such duality may be described as having an internal vs. external focus - focusing primarily on improving efficiency of internal operations vs. focusing externally on customer relations. This internal/ external focus could also manifest itself as being reactive to customer needs as opposed to proactive. 
With respect to the scope of implementation of TQM in a firm, there are firms that focus primarily on downstream plant-floor operations and those that strive to truly implement TQM company-wide.

Today, arguments as to whether TQM is a failure or success, dead or alive seem to have shifted to a more productive question: "What is the role of quality management in business today?" At least one author has stated that quality is now simply a 'qualifying criterion' (Prajogo and Sohal [25] citing Hill [26]). The apparent result of the quality movement, and TQM by extension, has been that dramatic improvements in quality resulted in dramatic increases in customer expectations. For most companies, quality is thus necessary but not sufficient as an element of competitive strategy. Globalization of markets and rapid economic growth in developing nations are forcing firms in developed nations to look to new sources for competitive advantage. Innovation is now widely recognized as a key factor to longterm competitiveness [27].

For managers, the question then becomes where and how to focus - quality or innovation? Of course, it is not an either/ or decision. If quality is a qualifying criterion for staying in business, managers must learn how to manage for quality and innovation simultaneously. However, are the two compatible? Are there trade-offs involved, i.e., if a firm focuses on quality will innovation necessarily suffer or viceversa? Quality programs have emphasized stability and efficiency, that is, 'doing it right the first time.' Further, quality programs typically focus on satisfying existing 
customers. Conversely, innovation requires flexibility and effectiveness, in other words, 'doing right things.' Innovation is often focused on attracting new customers; innovative firms acknowledge the risk of paying too much attention to satisfying existing customers at the expense of neglecting to stay attuned to the changing business environment. For these reasons, some say that a quality-oriented culture in a firm may be counter-productive to fostering a culture focused on innovation $[24,28$ 32]. Further, how can a manager choose between the right balance of the hard and soft factors, internal and external focus, implementation in downstream activities or upstream, etc.

Hence, for managers today, the primary questions related to quality and innovation are:

Can a company manage for quality such that innovation is positively impacted as well?

Can existing quality programs be adapted to also address innovation performance? If so, how, that is, what are the 'best practices?'

\section{$\underline{\text { Problem Definition }}$}

Researchers have only recently begun to explore relationships between quality management and innovation performance. As with research exploring the impact of TQM on quality performance, the results of research exploring the impact of TQM on innovation performance have been mixed as well. Singh and Smith concluded that there was insufficient evidence to link TQM and innovation [33]. Kanji states that successful innovation depends on TQM although this premise is not tested [34]. 
Studies by Prajogo et al. have shown positive correlations between TQM and innovation performance $[25,30]$. However, these same researchers have conducted research showing no significant relationships between quality and innovation performance when other factors such as technology management and R\&D management were also considered [35].

Even for studies showing a positive correlation between TQM and innovation performance, it is not yet clear which of the many approaches to implementing TQM (e.g., selection of, and emphasis on, specific principles and practices) lead to enhanced innovation performance. To date, research addressing the importance of the contingency approach to implementing TQM has emphasized relationships to quality performance rather than innovation performance [19, 20,36-47]. Sitkin et al. touched on the topic of innovation performance in discussing a contingency approach to implementing TQM by proposing two separate approaches: Total Quality Control (TQC) and Total Quality Learning (TQL) [24]. Choice of approach is dependent on the level of uncertainty in the firm's environment. TQC firms emphasize satisfying existing customers and exploiting existing skills. TQL firms emphasize scanning for new customers and exploring new skills and resources.

'Control vs. learning' is only one of many possible dualities discussed in the literature related to TQM implementation. As mentioned previously, implementation of TQM also varies in the nature of the principles and practices used. Lewis et al. 
examined the use of 'hard' (analytical) factors as opposed to 'soft' (human-centered) factors; the hard and soft factors were defined as:

\section{Hard Factors}

- Continuous improvement and innovation

- Information and performance measurement

- Process management

- Strategic planning

- Process control

- Product and service design

- Benchmarking

- Flexibility

- Quality systems

- Quality assurance

- Just in time

- Zero defect

Soft Factors

- Customer focus and satisfaction

- People training

- Top management commitment

- Teamwork

- Employee involvement

- Supplier management

- Communication

- Rewards and recognition

- Human resource management

- Employee empowerment

- Quality culture

- Employee satisfaction

- Social responsibility

Their research concluded that a holistic approach was best to ensure proper TQM implementation [48, 49]. 
Other researchers have discussed an internal vs. external focus to TQM implementation $[23,25,30]$. Internally-focused efforts emphasize improving operational efficiency whereas externally-focused programs place greater emphasis on customer relations. This internal/ external focus could also manifest itself as being reactive rather than proactive with respect to meeting customer needs. Reactive firms, while they may seek to understand customer needs, focus primarily on meeting current needs. Proactive firms work to educate customers about, for example, how new products could address latent needs (i.e., assisting customers to 'imagine the possibilities') [25, 28-30]. Additionally, within the concept of external focus, there are firms that take what could be considered a 'closed system' view of the firm's external environment, namely entities within the firm's supply chain. Firms that take more of an 'open system' view allow for consideration of a much broader picture of the company context. Such a view might include end consumers (for firms that do not sell direct to consumers), the communities where their products will be used, environmental impacts, etc.

Scope of implementation of TQM in a firm also varies; quality tools and techniques were initially focused primarily on downstream plant-floor operations. Over time, some firms expanded their quality management programs to include upstream operations such as design, sales and marketing, and accounts payable.

In summary, in addition to a multitude of principles and practices within the 'TQM toolbox', researchers have also identified a myriad of approaches to 
implementing TQM. The fact that TQM has had mixed results is not surprising; TQM has often been presented as a 'turnkey' philosophy for quality management. Overzealous proponents have gone so far as to label as heretics anyone that would suggest TQM may not be applicable to all situations [24]. It is little wonder that managers see each new philosophy that comes along as the 'fad of the month.' Exhortations on the importance of innovation (as an 'add on' to quality) to firms' future competitiveness, if not even survival, likely serve only to exacerbate the confusion and frustration. Even for firms that see value in continuing to invest in TQM, how can managers decide which of the many permutations of TQM to implement?

From the standpoint of evaluating the extent of implementation of TQM in firms, key limitations in the existing research have been the inability to capture the key contextual differences for firms combined with the difficulty in addressing the multitude of performance outputs desired from a quality management system. To address the first issue, there is growing recognition that there is no single approach to TQM that can accommodate the vast differences in context and strategy that exist from firm to firm $[50,51]$. How companies approach implementing TQM varies, depending on the industry, the level of maturity of their product, their strategy and objectives, organizational culture, and a host of other factors. However the measurement instruments currently used to evaluate the extent of TQM implementation in a company do not take into account how 'efficiently' or how well the tools are used. For example, a firm may report that they use statistical process control (SPC) to a limited extent. Regardless, they may make very effective use of 
SPC by using it only where it gains them the most for their effort. Conversely, another firm may report they make extensive use of SPC, which may simply be a symptom of taking a 'shotgun approach' to using quality tools. As a result, compared with the other firm, they may feel that they get very little payoff given their investment. There has been little if any research examining the relationship between quality management and performance that adequately addresses this challenge.

Further, as will be discussed below, there are multiple definitions and perspectives of quality. Early research in the field primarily examined quality from the perspective of "conformance to specifications." Researchers and practitioners increasingly recognized the fallacies of this view in that it fails to address the multidimensional nature of quality. Conformance to specification is one key dimension of quality; others include reliability, durability, and customer satisfaction. Further, for researchers interested in exploring the impact of quality on organizational performance, there are numerous potential dimensions that could be explored with respect to organizational performance. In today's business environment, many have argued that innovation must be added to the growing list of essential organizational performance metrics [52].

However, evaluating and comparing performance within and between organizations can be quite difficult when there are multiple, inter-related dimensions to performance. Data Envelopment Analysis (DEA) is a non-parametric approach to productivity and efficiency analysis that allows for simultaneous comparison of 
multiple inputs and multiple outputs [53]. It is uniquely suited to the situation at-hand here as it would enable the examination of multiple quality inputs and multiple performance outputs. In the context of efficiency, its use here would be on the 'efficiency' of use of principles and practices of quality management; that is, what benefit (outputs) are firms getting given their investments? Further, DEA would enable the analysis to take into account the contextual variation described above for firms' quality management systems.

Further, the results of DEA analysis can be combined with the case study method to provide rich descriptions of the specific practices that benchmark firms use to achieve superior performance. Specifically, the DEA results will point to firms that are efficiently converting quality management inputs into quality and innovation performance outputs, i.e., firms that have focused on specific practices as opposed to firms taking a 'shotgun approach' to quality management. Case studies with both groups of firms can then be conducted to provide deeper insights into the best practices.

In summary, by identifying best quality management practices to achieve improved performance (specifically quality and innovation performance), managers will be better able to design quality management systems that enable them to simultaneously address two of the primary competitive factors in business today. 
A point must be made here regarding the decision to focus principally on TQM; to this point, only passing mention has been made of other approaches to quality management. When examining the impact of quality management systems on performance, shouldn't other approaches like Business Process Reengineering (BPR), Six Sigma, and ISO 9000 also be given due consideration? As discussed previously, there is disagreement in the research community as to whether BPR and Six Sigma are truly separate and distinct from TQM. Furthermore, also as discussed previously, there is no universally-accepted definition of TQM. Many authors have made the point that the label itself is not what is important but the practices and the underlying principles guiding their use. For example, Prajogo and Brown examined the performance impacts of formal vs. informal TQM programs and concluded that it is more important to implement TQM as a set of practices than to be concerned with the label [20]. What is proposed here is just that $-\mathrm{a}$ focus on the adaptation of a set of principles and practices to quality management. At the same time, the convention of the literature in quality management systems has been to label all company-wide or systems-based approaches to quality management as TQM regardless of the vast differences in implementation.

With respect to the ISO 9000 series of standards, the choice was made not to explicitly address these standards. The original standards and 1994 revision were slanted toward documentation and verification of processes rather than emphasizing performance. The European Union called for de-emphasis of ISO 9000 registration because it was felt firms were more concerned with "passing a test" than on quality 
improvement [2]. It may be that there has not been sufficient time such that changes made to the most recent version of the standards (ISO 9000:2000) to address these weaknesses have become evident. However, research by Prajogo and Brown revealed that "...organizations that had focused solely on ISO 9000 did not produce any noticeable performance benefits", whereas "broader approaches" to quality such as TQM did produce better quality outcomes [44].

For these reasons, the term TQM is used here synonymously with broad-based (company-wide) quality management systems in general. Again however, it is the specific principles and practices of quality management that will be examined rather than simply the impact on performance of 'applying the TQM label.' 


\section{Chapter 2. Literature Review}

For the research conducted here, the relevant literature includes topics related to definitions of quality, TQM, innovation, and measurement constructs. These topics are presented followed by an overview of the proposed research methods, Data Envelopment Analysis (DEA) and the case study method. The section concludes with a review of the literature exploring the relationships between quality and performance.

\section{Quality}

To understand the roles quality plays in an organization, one must first understand how the term is defined. This is much easier said than done; quality can be defined from numerous perspectives and in both objective and subjective terms. The American Society for Quality (ASQ) defines quality as:

"A subjective term for which each person has his or her own definition. In technical usage, quality can have two meanings: 1 . the characteristics of a product or service that bear on its ability to satisfy stated or implied needs. 2. a product or service free of deficiencies." [54]

This definition explicitly recognizes the subjective element as well as at least two dimensions - customer needs and conformance to specifications (i.e., absence of defects). Garvin's five approaches to defining quality reveal some additional dimensions of quality [55]: 
1. Transcendent is synonymous with "innate excellence"; quality traits are an absolute and universally recognized. Thus, quality cannot be defined precisely but "you just know it when you see it." [2] The primary criticism of this view is that it does not provide a means to measure quality and hence for managers to make decisions.

2. Product-based views quality of a product as represented by some measurable attribute. For example, the quality of a car could be reflected by its horsepower rating and/or mileage. Although this approach to defining quality is quantitative, it suffers from the fact that the value of the specific attributes to individual customers will be highly variable.

3. User-based is in essence the 'customer is always right' view of quality. This is a highly subjective view given that each customer determines how quality is defined. Another way of stating this view is 'fitness for intended use.' [2]

4. Manufacturing-based is the analytical, and most objective view of quality in that it can be summarized as conformance to specifications; hence it is often called 'conformance quality.' This view of quality was the predominant definition in industry for the early decades of the quality movement. This definition underlies the approach to quality that emphasizes data-based decision making and management by fact. However, like the product-based view, a weakness to this view of quality lies in the differing value individual customers ascribe to different product attributes. Taken to an extreme, a firm can have near perfect conformance to a specification that makes no difference to their customers.

5. Value-based relates usefulness or satisfaction to price. This view of quality recognizes the need for balance - excellence at a fair price (customer, i.e., external view) and conformance to specifications at a reasonable cost (firm, i.e., internal view).

Based on these views, Garvin proposed eight dimensions to quality:

conformance to specifications, product reliability, product durability, design quality, product improvement, brand image, company reputation, and customer service [55]. 
According to Evans and Lindsay, by the end of the 1980s, many companies began defining quality as "meeting or exceeding customer expectations" [2]. This definition reveals a shift in philosophy from product-focused to customer-focused.

How firms perceive and define quality naturally impacts their approach to quality management. In the early years of formal quality management programs, quality was primarily viewed through the lens of Garvin's manufacturing-based definition. That is, quality was equated with conformance to specifications. Thus, approaches to quality management centered on analytical methods to measure, monitor, and control specific process variables. Analytical tools like statistical process control (SPC) developed in the late 1920s in the U.S. telecommunications industry were the cornerstone of such approaches to quality [56]. The emphasis on statistical methods to improve quality in manufacturing operations were the primary focus of quality programs from the 1930s through WWII; emphasis in manufacturing was on producing consistent and reliable goods for the war effort.

Following the war, quality gurus like W. Edwards Deming and Joseph M. Juran rose to prominence in Japan as their quality tools and philosophies guided efforts to rebuild Japan's post-war economy. During this time, recognition of the need for a holistic, systems view of quality began to grow. In the 1950s, Armand Feigenbaum coined the term 'Total Quality Control'; the Japanese adopted this view and termed it 'companywide quality control' [2]. The roots of TQM were thus established; however the quality movement had yet to take hold in the U.S. 
The rapid development of quality methods in Japan following the war had a dramatic and positive impact on the quality of Japanese goods. As export of these goods increased, quality rose in importance as a source of competitive advantage. In 1983, Garvin conducted a groundbreaking study comparing U.S. and Japanese manufacturers of room air conditioners [57]. He reported that “...the poorest Japanese company (with respect to quality) typically had a failure rate less than half that of the best U.S. manufacturer.” By the 1980s, U.S. industry, automakers and electronics firms in particular, were feeling significant pressure to improve quality [58]. TQM as a management philosophy spread rapidly around the globe in the 1980s [59].

In summary, quality can be defined via both subjective as well as objective measures. In the early years of the quality profession, the predominant measures were objective, specifically conformance to specifications. As the profession has changed through time, however, practitioners and researchers alike have increasingly recognized the inherent multidimensionality of quality and hence the need to accommodate subjective measures as well. Further, the focus has shifted from an internal and product-focused view of quality (e.g., defects) to an external focus and customer-focused view. This is evident by the common definition of quality, "meeting or exceeding customer expectations."

With the preceding as background on quality in general, the focus is now shifted to more precisely defining TQM, the fundamental principles, key criteria, as 
background for discussing key constructs and tools to measure extent of implementation of TQM in a firm.

$\underline{\text { TQM }}$

As difficult as it is to define quality, it is equally if not more difficult to define TQM. Numerous authors have attempted to provide a simple definition. Forker et al. define TQM as “.... an integrated system of principles and procedures whose goal is to improve the quality of an organization's goods and services" [38]. Flynn et al. define TQM as, "an integrated approach to achieving and sustaining high quality output, focusing on the maintenance and continuous improvement of processes and defect prevention at all levels and in all functions of the organization, in order to meet or exceed customer expectations" [60]. These definitions include key principles of continuous improvement, the inclusion of all functions/ integration, and focus on the customer. In this vein, Evans and Lindsay state that Total Quality is based on three fundamental principles:

1. A focus on customers and stakeholders;

2. Participation and teamwork by everyone in the organization; and

3. A process focus supported by continuous improvement and learning [2].

By today's standards, these principles may seem quite commonplace.

However, when the philosophy of TQM was in the developmental stages, quality was the job of the quality department and consisted of ensuring products met specifications [2]. Teamwork was by and large unheard of as was the focus on understanding customer needs and requesting customer feedback. Thus, TQM was a radical 
departure from contemporary management philosophies and practices. One apparent success of TQM has been to make these fundamental principles the current modus operandi for many businesses around the globe. Even so, the point has been argued that many failures of TQM to positively impact quality performance have been traced to failures in execution, i.e., inadequate attention given to one or more of these fundamental principles and to adapt the principles to company strategy, culture and context $[22,46]$.

Sitkin et al. discuss a slightly different set of three fundamental principles underlying TQM [24]. While they also mention a focus on customer satisfaction and continuous improvement, they essentially blend the principles of process focus and teamwork into a third principle defined as "treating the organization as a total system." This systems view of quality management is important to state explicitly in that one of the evolutionary improvements to TQM has been to expand the view of the system from encompassing 'sales to shipping' (i.e., the internal view) to include the company, its suppliers, and its customers (the external view) and even beyond to communities and the environment [61].

While the three principles described above serve as a simple means to define the guiding principles of TQM, they lack sufficient detail to define key principles and practices used in firms to implement TQM. The U.S. Malcolm Baldrige National Quality Award (MBNQA) is often used as the conceptual framework for defining the key principles and practices $[62,63]$. 
The MBNQA was developed following a national study on productivity initiated by President Reagan in 1982. The Malcolm Baldrige National Quality Improvement Act was signed into law in 1987. The program focused on stimulating quality and productivity in American companies; recognizing achievements of exemplary companies; establishing guidelines for firms to evaluate their quality improvement efforts; and providing guidance to other companies via publishing information on the practices of award-winning firms. The award is named in honor of the U.S. Secretary of Commerce who was killed in an accident prior to the act being signed into law. The National Institute of Standards and Technology administers the MBNQA [2].

The seven criteria that comprise the MBNQA include [61]:

1. Leadership - how senior leaders guide and sustain an organization as well as governance of the firm and how ethical, legal, and community responsibilities are addressed.

2. Strategic planning - how strategic objectives and action plans are developed as well as how they are deployed and changed; how progress is measured.

3. Customer and Market Focus - how the requirements, needs, expectations, and preferences of customers and markets are determined; how relationships with customers are built and the key factors leading to customer acquisition, satisfaction, loyalty, retention and sustainability.

4. Measurement, Analysis, and Knowledge Management - how information and knowledge assets are selected, gathered, analyzed, managed, and improved as well as how performance is reviewed.

5. Workforce Focus - how work systems, employee learning, and motivation enable the development and utilization of employees' full potential in alignment with strategic objectives and action plans; how the organization builds and maintains a work environment and climate for employee support 
that leads to performance excellence and to personal and organizational growth.

6. Process Management - examination of all key product, service, and organizational processes for creating customer and organizational value and organizational support.

7. Results - examines performance and improvement in product and service outcomes, customer satisfaction, financial and marketplace performance, human resource outcomes, operational performance, leadership, and social responsibility; performance is examined relative to competitors and other organizations with similar products and services.

Figure 1 presents a framework for visualizing the interrelationships among the seven MBNQA criteria.

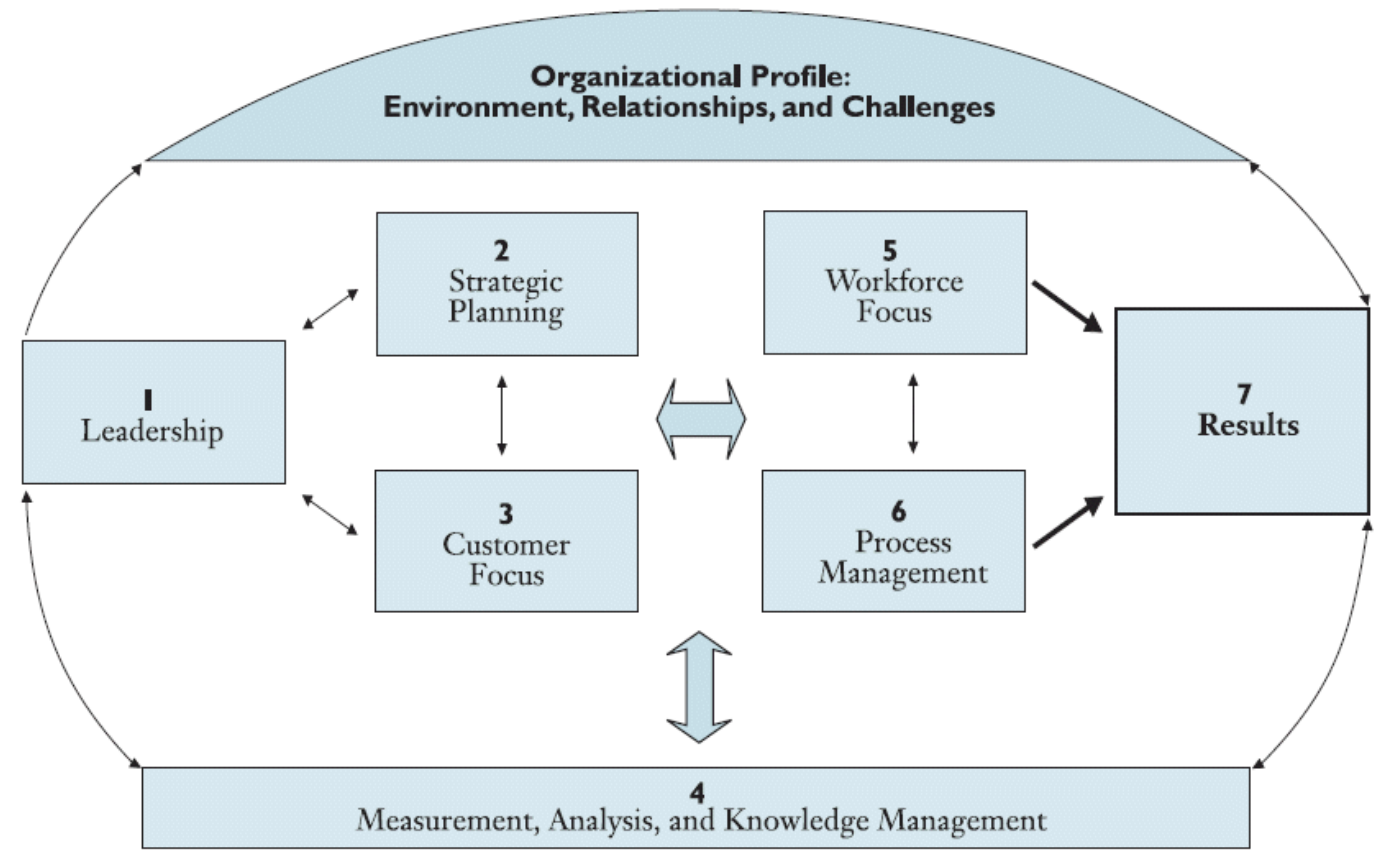

Figure 1. Baldrige Criteria Framework: A Systems Perspective [61]

As can be seen, the focus of this award extends far beyond the traditional narrow view of conformance quality (meeting specifications) to performance 
excellence and takes a systems view of the firm. As discussed previously, BPR developed in part due to a criticism of TQM to focus too heavily on incremental improvement. One of the changes to the MBNQA criteria was to expand the definition of improvement to include breakthrough improvement [31]. For example, the MBNQA section on Measurement, Analysis, and Knowledge Management poses the question to award applicants - "How do you translate organizational performance review findings into priorities for continuous and breakthrough improvement and into opportunities for innovation?"

In summary, like quality, TQM is difficult to define. However, the three fundamental principles of process focus/ continuous improvement, customer focus, and participation and teamwork capture the essence. Further, the seven criteria of the MBNQA are widely recognized as providing a framework for assessing the use of TQM in a firm and its impact on organizational performance. To understand how TQM has changed since its inception, it is important to understand some of the key dualities within TQM and how these affect implementation.

\section{Dualities within TQM}

Several TQM researchers have addressed the concept of dualities or dichotomies within TQM. The concepts may also be viewed as continuums, but for purposes of discussion here, the term duality, as originally used by Sitkin with respect to the 'control vs. learning duality' within TQM [24] will be used. 
Perhaps the most widely studied duality is 'hard vs. soft' factors ${ }^{1}$. Wilkinson was among the earliest authors to note that insufficient attention was being given to the 'soft', or human resource-related issues within TQM [64]. Lewis et al. explored the soft vs. hard factors. Soft factors were defined as including customer focus and satisfaction, people training, top management commitment, teamwork, employee involvement, and supplier management. Hard factors include continuous improvement and innovation, information and performance measurement, process management, strategic planning, process control, and product and service design [48]. These authors also advocate for a holistic approach to implementing TQM; firms using the ISO 9001 quality system standard as a means to implementing TQM were giving insufficient attention to the soft factors. Dow et al. explored the impact of specific TQM practices on quality performance and concluded that hard factors (defined as benchmarking, cellular work teams, advanced manufacturing technologies, and close supplier relations) did not contribute to superior quality performance whereas the soft factors did [65]. Citing Kekale and Kekale [22], Prajogo et al. captured this issue well in stating that “...perceiving TQM narrowly as a set of tools and techniques (i.e., hard aspects) has proven to be one of the primary failures of TQM implementation" [30].

A second type of duality within TQM is the scope of implementation of TQM in a firm. From the start, the 'Total' in Total Quality Management indicated the goal

\footnotetext{
${ }^{1}$ Note: The terms hard vs. soft, while imprecise, are the convention in the literature for distinguishing between the analytical/ technical/ quantitative tools and techniques and the more human-focused/ qualitative tools and techniques, respectively.
} 
of focusing quality improvement efforts company-wide [2]. Regardless, years of quality efforts being focused solely on the plant floor, combined with challenges in adapting quality methods to non-manufacturing applications, led to slow spread of TQM throughout organizations. As such, TQM programs that were narrower in scope were such that quality continued to be the responsibility of the quality department; involvement of other business functions and personnel was minimal. Firms with strong senior management leadership and commitment to TQM focus truly companywide and include upstream operations such as product design, sales and marketing, maintenance, shipping, and accounts payable in addition to downstream manufacturing operations [2]. Along these same lines, McAdam et al. state that the literature on TQM divides TQM into two categories - holistic TQM and continuous improvement TQM [23].

The third duality within TQM involves issues related to internal vs. external focus. Internally-focused efforts emphasize continuous process improvement whereas externally-focused programs place greater emphasis on customer relations. These are of course, two of the three fundamental principles of TQM presented above. Thus, a balanced approach to implementing TQM would give equal attention to both principles.

Internal/ external focus can also manifest itself as being reactive rather than proactive with respect to meeting customer needs. Reactive firms include not only firms that react in the sense of waiting for customers to complain before they take 
action, but also firms that seek to understand customer needs. The primary issue is that the firms focus primarily on meeting current needs. Proactive firms work to educate customers about, for example, how new products could address latent needs (i.e., assisting customers to 'imagine the possibilities') [24]. Additionally, within the concept of external focus, there are firms that take what could be considered a 'closed system' view of the firm's external environment, namely customers and suppliers with whom they have direct business relationships. Firms that take more of an 'open system' view allow for consideration of a much broader picture of the company context. Such a view might include end consumers (for firms that do not sell direct to consumers), the communities where their products will be used, and environmental impacts [24].

Figure 2 presents a graphical model of the three fundamental TQM principles discussed previously. As these principles are fundamental, successful implementation requires giving adequate attention to all three principles. 


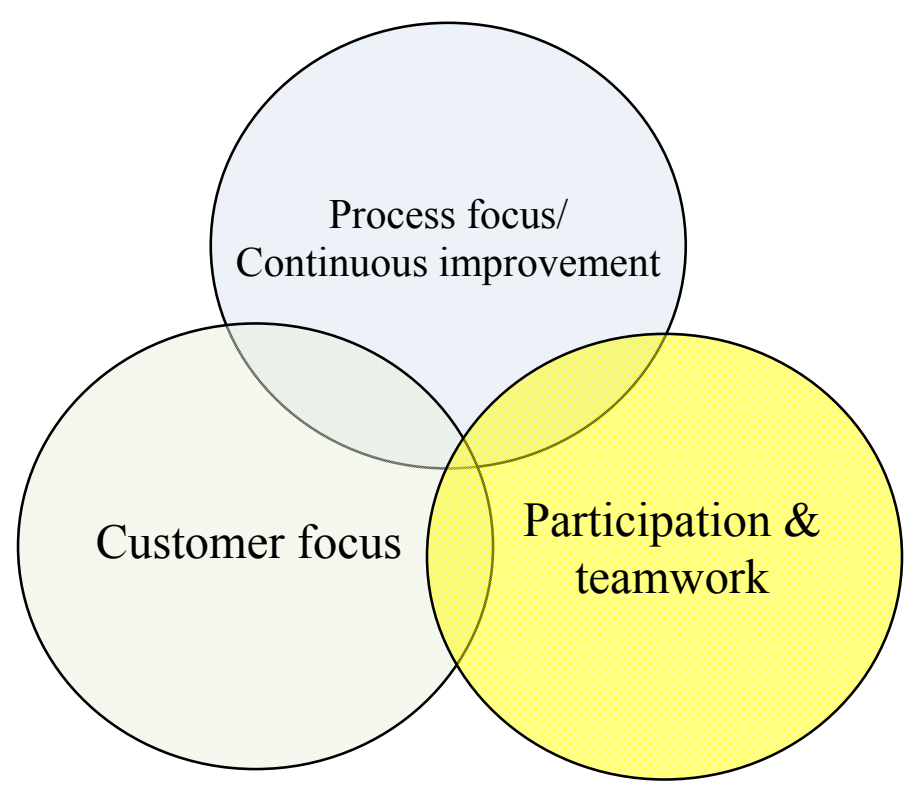

Figure 2. Three Fundamental Principles of TQM

In practice, the dualities discussed above can be thought of as an 'imbalance' in the emphasis on the three fundamental TQM principles. For example, the hard vs. soft factor duality can be considered a focus on continuous process improvement (which typically involves the use of the hard tools like SPC and design of experiments) vs. the other two factors that typically involve more of the soft factors.

Narrow scope of implementation results from focusing primarily on continuous process improvement and, to a lesser extent, customer focus. TQM in these firms involves personnel in the quality function focusing on process improvement and often reacting to customer complaints (a weak 'intensity' of customer focus). TQM efforts that are broader in scope add to the process improvement principle by taking a systems 
view of the firm's processes and hence include upstream as well as downstream operations. Broader scope can also lead to an expanded view of customer focus. As a result, broadening the scope requires company-wide participation and teamwork.

The internal vs. external duality results primarily from focusing on the internal issues of continuous process improvement and perhaps participation and teamwork vs. the external issues related to customer focus. This duality can also be considered in light of the intensity of customer focus. As with the implementation scope, the intensity of focus on the practices within the customer focus principle will be higher in firms with more of an external than internal focus.

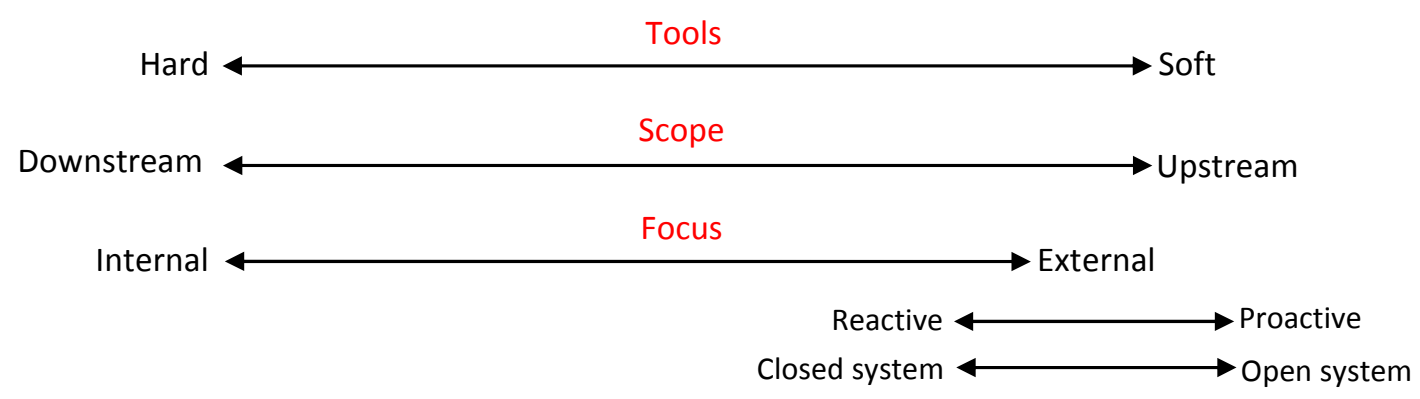

Figure 3. Dualities within TQM Implementation

Figure 3 presents these dualities as a series of continuums. Further, the endpoints are aligned in an attempt to demonstrate the evolution of TQM. That is, TQM initially focused on the use of hard tools for process improvement, primarily in downstream manufacturing operations. Over time, there has been increasing recognition of the need to focus on the 'human side' of quality (i.e., the soft tools); to more fully embrace a customer focus (and to be proactive rather than reactive and to 
take a more open view of the system to including needs of potential new customers); and to expand the scope of TQM to be truly 'Total' Quality Management - i.e., to use the principles and practices in upstream as well as downstream operations.

In summary, there are several well-established dualities within TQM including use of hard vs. soft tools and techniques, internal vs. external focus, and scope of implementation. The dualities may be conceived of as varying levels of emphasis on the three fundamental principles of TQM of process focus/ continuous improvement, customer focus, and participation and teamwork. Failures of TQM to positively impact performance have been traced to failures of execution; such failures can often be traced to inadequate attention being given to one of the three fundamental principles. Further, and a key point underlying these dualities, is that there is no single approach to implementing TQM; approaches can be expected to vary from firm to firm. Where a firm producing commodity products might place a great deal of emphasis on practices that lead to conformance quality (i.e., ensuring products meet specifications) another firm focused on growing market share by introducing new products might place more emphasis on customer focus. Ideally, decision makers would have the information available to them to know where and how to focus quality management efforts to achieve the objectives suited to their particular context. Additionally, decision makers need to know how to adapt quality management efforts to achieve new areas of emphasis such as innovation performance. However, prior to determining what to change, organizations must have some means of evaluating 
current extent of implementation of the various principles and practices of TQM, i.e., a quality management measurement instrument.

\section{Quality Management Constructs and Measurement Instruments}

Within a decade of the 'explosion' of TQM in the 1980s came an implosion of sorts. Reports of failures of TQM to produce results began to hit the popular press in the 1990s [3]. In his address to the 1993 annual conference of the American Society for Quality, Senge reported that less than a third of the 500 TQM firms surveyed were accomplishing anything; and two-thirds of the TQM programs had ceased to function [8]. In the $90 \mathrm{~s}$, researchers began in earnest to explore the relationships between TQM and organizational performance. However, it quickly became apparent that a measurement instrument was needed that would enable researchers to distinguish TQM from non-TQM firms as well as to quantify the level or intensity of implementation of the various principles and practices within TQM.

As discussed above, the MBNQA criteria are often used as the conceptual framework for quality management. Flynn et al. state, "Use of the Baldrige criteria is a way of judging the face validity of any framework which might be proposed for quality management" [60]. In this regard, it might seem that researchers and practitioners could simply use the award criteria as a measurement instrument to assess the extent of TQM implementation in a firm rather than 'recreating the wheel.' However, the award criteria are not intended to serve as a measurement instrument per se. Rather, they are intended to elicit narrative responses regarding how applicant 
firms address each of the seven primary MBNQA criteria described above and shown in Figure 1. For example, for the Customer and Market Focus criterion, applicants are asked, "How do you identify customers, customer groups, and market segments? How do you predetermine which customers, customer groups, and market segments to pursue for current and future products and services? How do you include customers of competitors and other potential customers and markets in this determination?" [66] Thus, while the MBNQA may not serve as an instrument to provide a score for extent of implementation of various quality management principles and practices, many researchers have used the criteria to guide the development of such measurement instruments.

Saraph et al. were among the first to develop a quality management measurement instrument [67]. These authors recognized that quality data such as defect rates, error rates, rework cost, etc. are not measures of organization-wide quality management. The authors reviewed a large body of quality literature including the philosophies of well-known quality experts such as Crosby, Deming, Garvin, Ishikawa, and Juran. The principles proposed by these experts were synthesized to formulate critical quality factors. Data were collected from industry professionals to develop operational measures for each critical factor; measures were tested for reliability and validity. For reliability, Cronbach's alpha was used as a measure of internal consistency [68]. Following deletion of one to three items from each factor, the factors were deemed reliable (i.e., all scores were greater than the 0.70 threshold 
suggested by Nunnally [69] and Hair et al. [68]). The results were the following eight

critical factors and selected examples of perceptual measures within each factor:

1. The role of management leadership and quality policy - includes measures of extent to which top division executives assume responsibility for quality performance, specificity of quality goals, and degree of participation by major department heads in the quality improvement process.

2. Role of the quality department - includes measures of the visibility of the quality department, autonomy of the department, and amount of coordination between the quality department and other departments.

3. Training - includes measures of specific work-skills and quality-related training given to hourly employees, training in the 'total quality concept' (i.e., philosophy of company-wide responsibility for quality) throughout the division, and availability of resources for employee training.

4. Product/ service design - includes coordination among affected departments in the product/ service development process, quality of new products/ services in relation to cost or schedule objectives, and extent to which manufacturability is considered in the product design process.

5. Supplier quality management - includes measures of extent to which suppliers are selected based on quality rather than price or schedule, reliance on reasonably few dependable suppliers, and involvement of the supplier in the product development process.

6. Process management - includes use of acceptance sampling to accept or reject lots or batches of work, extent to which process design is 'fool-proof' and minimizes chances of employee errors, and degree of automation of the process.

7. Quality data and reporting - includes measures of availability of cost of quality data in the division, timeliness of the quality data, and extent to which quality data are available to hourly employees.

8. Employee relations - extent to which employee involvement programs are implemented in the division, extent to which employees are held responsible for error-free output, and amount of feedback provided to employees on their quality performance. 
The full measurement instrument is shown in Appendix A1 (Note: items deleted to improve reliability are not shown in the instrument).

Flynn et al. developed a framework for quality management research as well as a measurement instrument [60]. They built on the work of Saraph et al., but strove to overcome what they perceived as limitations of the previous work. Specifically, Flynn et al. felt that emphasizing plant-level implementation of quality was preferred to division-level implementation given their experience with occasional examples of exemplary plants within less than outstanding organizations. Further, Flynn et al. built their foundation on quality practices in actual use as opposed to building upon the theoretical work of quality gurus as was done by Saraph et al. Further, separate instruments were developed for direct laborers, plant managers, quality managers, etc. Measures were tested for reliability and validity. For reliability, Cronbach's alpha was used as a measure of internal consistency. Following deletion of one to four items from each of the factors, the measures were deemed reliable (i.e., all scores were greater than the threshold of 0.60 suggested by Nunnally [69] for new scales). The results were seven dimensions of quality management and eleven perceptual scales. Each scale included both perceptual as well as objective measures. The dimensions and scales include:

1. Top management support

a. Quality leadership

b. Quality improvement rewards

2. Quality information

a. Feedback

b. Process control 
3. Process management

a. Cleanliness and organization

4. Product design

a. New product quality

b. Interfunctional design process

5. Workforce management

a. Selection for teamwork potential

b. Teamwork

6. Supplier involvement

a. Supplier relationship

7. Customer involvement

a. Customer interaction

The complete measurement instrument is shown in Appendix A2.

Ahire et al. conducted a number of studies to compare quality management in TQM versus non-TQM firms and to develop implementation constructs [17, 50, 63]. Their research built on that by Saraph et al. and Flynn et al. and worked to overcome perceived limitations of the previous work. Specifically, the authors felt that Saraph et al. excluded two important constructs: customer focus and SPC. Interestingly, Saraph et al. initially included a variable on the use of SPC, however the item was dropped to improve instrument reliability [67]. This is a curious result given that SPC played such a critical role in the founding of the quality sciences and was synonymous with quality for many years. Further, Ahire et al. felt there were several tautologies in the Saraph et al. instrument. For example, including "commitment of the divisional top management to employee training" in the Training construct likely resulted in 
artificially high correlations among constructs due to overlap with the Role of Divisional Top Management and Quality Policy construct.

With respect to the Flynn et al. measurement instrument, Ahire et al. stated the two instruments were quite complementary. The principal differences are that Ahire et al. include constructs for Employee Empowerment and Benchmarking and omit the team-oriented scales and cleanliness and organization scale found in Flynn et al.'s instrument. Also, Ahire et al. opted for a construct on Customer Focus as opposed to customer interaction stating that higher customer interaction may in fact reflect poor quality management. This distinction could be drawn based on the impetus for the interaction, i.e., whether it was reactive (responding to complaints) or proactive (requesting feedback). Measures were tested for reliability and validity. For reliability, Cronbach's alpha was used in conjunction with the Werts-Linn-Jorsekog coefficient $\left(\rho_{c}\right)$. Following deletion of five (of eight) items from the Employee Involvement construct, the factors were deemed reliable, i.e., all alpha scores were greater than the minimum value of 0.70 suggested by Nunnally [69] and greater than 0.50 for $\rho_{\mathrm{c}}$. The authors acknowledge that deletion of this many items from a single construct may have resulted in problems with content validity.

The 10 constructs to measure integrated quality management presented, as well as two dimensions to measure performance (product quality and supplier performance) by Ahire et al. include: 
1. Top management commitment

2. Customer focus

3. Supplier quality management

4. Design quality management

5. Benchmarking

6. Statistical process control (SPC) usage

7. Internal quality information usage

8. Employee empowerment

9. Employee involvement

10. Employee training

11. Product Quality

12. Supplier performance

The complete measurement instrument is shown in Appendix A3.

Singh and Smith also contributed to the literature on quality management measurement instruments [70]. These authors propose a measurement instrument that addresses the perceived limitations of the instruments developed by Saraph, Flynn, and Ahire discussed above. The primary emphasis is on developing a tool that reflects the current state of quality practice, namely the three-pronged approach to quality management: standards-based (e.g., ISO 9000), prize-criteria (e.g., awards-based such as MBNQA), and the elemental approach (i.e., principles proposed by academicians and practitioners). Based on their review of the literature, Singh and Smith propose eight constructs within quality management and one construct to describe the business environment:

1. Top management leadership

2. Customers

3. Employees

4. Suppliers

5. Information and communication systems 
6. Processes

7. Wider community

8. Competitors

9. Business conditions

Reliability was assessed using maximal composite reliability coefficients. Coefficients exceeded the 0.60 threshold for all but two constructs - customers and suppliers - and these were only marginally outside the limit and were hence retained. Therefore, the authors deemed the measures to be generally reliable. The complete measurement instrument is shown in Appendix A4.

Motwani identified the critical factors and performance measures of TQM [71]. Critical factors were identified via blending the approaches used by Saraph, Flynn, and Ahire along with three other studies directed to develop TQM critical factors. Motwani's unique contribution to the research in TQM constructs is the development of quantitative, in addition to qualitative performance measures for each of the constructs as shown in Table 1.

While there is certainly value in having quantitative, objective measures for the critical factors in TQM in order to develop a score for "extent of implementation", many of the measures listed by Motwani are not readily obtainable from objective data. Further, these measures are merely proposed in the research and not validated or tested in empirical research. 
Table 1. Performance Measures for Each Critical Factor of TQM [71]

\begin{tabular}{|c|c|}
\hline Critical factor & Performance measures \\
\hline $\begin{array}{l}\text { Top management } \\
\text { commitment }\end{array}$ & $\begin{array}{l}\text { Allocate budget and resources ( } \$ \text { or hours per employee) } \\
\text { Control through visibility } \\
\text { Monitor progress } \\
\text { Planning for change }\end{array}$ \\
\hline $\begin{array}{l}\text { Quality measurement and } \\
\text { benchmarking }\end{array}$ & $\begin{array}{l}\text { Zero-defects conformance } \\
\text { Use SPC for process control } \\
\text { Cost of quality } \\
\text { Proportion of defects } \\
\text { Percentage of products needing rework } \\
\text { Defective rate relative to competitors }\end{array}$ \\
\hline Process management & $\begin{array}{l}\text { Unit cost } \\
\text { Production goals } \\
\text { Reduce material handling } \\
\text { Design for manufacturability } \\
\text { Reduce cycle time } \\
\text { Reduce setup time } \\
\text { Productivity = finished good/ no. of people or production } \\
\text { hours } \\
\text { Productivity = total process time/ total delivery time }\end{array}$ \\
\hline Product design & $\begin{array}{l}\text { Number of new products introduced } \\
\text { Time taken from design to first sale } \\
\text { Fitness of use } \\
\text { Design quality }\end{array}$ \\
\hline $\begin{array}{l}\text { Employee training and } \\
\text { empowerment }\end{array}$ & $\begin{array}{l}\text { Training employees ( } \$ \text { or hours) } \\
\text { Training management ( } \$ \text { or hours) } \\
\text { Cross-training employees } \\
\text { Training/ retraining budget }\end{array}$ \\
\hline Vendor quality management & $\begin{array}{l}\text { Reduce inventory } \\
\text { Supplier relations } \\
\text { Number of suppliers } \\
\text { Inventory turnover } \\
\text { Inventory accuracy } \\
\text { Implement kanban } \\
\text { Material cost } \\
\text { Material availability }\end{array}$ \\
\hline $\begin{array}{l}\text { Customer involvement and } \\
\text { satisfaction }\end{array}$ & $\begin{array}{l}\text { Delivery dependability } \\
\text { Operators involved/ value-added labor } \\
\text { Customer service training budget } \\
\text { Prompt handling of complaints } \\
\text { Number or percent of complaints } \\
\text { Number or percent of complaints that are delivered late } \\
\text { Broad distribution channels } \\
\text { Number of contacts with customers } \\
\text { Consumer surveys } \\
\text { Time to respond to questions/ complaints } \\
\text { Responsive repairs } \\
\text { Percentage of repeat business }\end{array}$ \\
\hline
\end{tabular}


Samson and Terziovski also developed a TQM implementation measurement instrument [46]. These authors used the MBNQA criteria to develop TQM implementation constructs and used the measurement instrument developed to explore the relationships between TQM practices and operational performance. Reliability was assessed using Cronbach's alpha and, following deletion of a total of eight variables, five of the seven constructs exceeded the 0.70 threshold. Two constructs, Process Management and Organizational Performance, fell slightly short of the 0.70 threshold (0.697 and 0.674 , respectively) and were retained rather than reduce the number of indicators. Given that the authors also used the instrument to explore relationships between TQM and organizational performance, a more detailed summary of the paper will be presented below.

In summary, the measurement instruments to assess extent of implementation of various quality management principles and practices have evolved to address limitations of prior generations of instruments and to stay current with quality management practices. The instruments available to-date are not without limitations. For example, certain principles such as measurement of customer satisfaction and a proactive approach to customer focus are addressed to a limited extent, if at all. Key tools (e.g., SPC) are omitted from a few of the instruments or have been deleted to improve instrument reliability. Again, this is curious given that tools like SPC played a prominent role in the early stages of TQM. These limitations aside, the fact that the instruments have, with the exception of Motwani's [71], all been tested and found to 
be reliable and valid provides assurance that they are adequate for purposes of measuring extent of implementation of quality management in an organization.

Having discussed the varying definitions of quality and TQM, underlying principles of TQM and measurement instruments for assessing extent of implementation of TQM, the discussion is now shifted to a similar analysis of innovation - how it is defined and measured, as well as key areas of commonality with quality management.

\section{$\underline{\text { Innovation }}$}

In common usage, the term innovation often connotes a new high-tech device or 'gadget'; the terms technology, invention, and innovation are often used synonymously [72]. In this regard, innovation in a firm would be primarily the purview of the R\&D department. However, the literature on innovation reveals that the research community conceives of innovation much more broadly.

Betz contrasts innovation with invention by defining invention as “...the creation of a functional way to do something, an idea for a new technology" [73]. By contrast, innovation is “...introducing a new or improved product, process, or service into the marketplace." In short, the output of invention is knowledge and the output of innovation is the economic benefit derived from the commercialization of the invention [73]. In Betz's view, both terms center on the idea of creation of something new. 
Damanpour defines innovation as "the adoption of an idea or behavior new to the adopting organization" [74]. Similarly, in the book Diffusion of Innovations, Rogers defines innovation as, “.... an idea, practice, or object that is perceived as new by an individual or other unit of adoption" [75]. Thus, these authors focus on adoption rather than creation of innovation.

The key similarities in these definitions are the emphasis on 'newness' or novelty. In fact, in addressing the question, "What is innovation and how should it be operationalized?", Johannessen et al. proposed that 'newness' was the common denominator for the various definitions of innovation [76].

While newness is a common denominator for innovation, one must ask specifically what is new? As stated previously, innovation is commonly thought of as new products, however numerous authors agree that innovation is not limited to products. For example, Betz's definition of innovation includes process and service dimensions of innovation in addition to product. And taking the typology a step further, Utterback adds administrative and technological innovations [77]. Administrative innovations are changes that affect policies, allocation of resources and other factors associated with social structures; they are indirectly related to the basic work activity of an organization and directly related to its management. Technological innovations pertain to products, services, and the technology used to produce products or render services and are directly related to the basic work activity of an organization. From this latter definition, there seems to be significant overlap 
between technological innovations and process innovations; specifically, technological innovations facilitate process innovations (regardless of whether it is an administrative process or a manufacturing process). Such overlap would likely lead to challenges in measuring process innovations as distinct from technological innovations.

Hovgaard and Hansen define three basic types of innovations - product, process, and business systems [52]. In their view, business systems innovations include any innovation that does not fall under product or process innovation. Examples include innovative management and marketing techniques. This definition appears to combine the administrative and the service-focused aspects of the technological innovations in Utterback's definition. The Hovgaard and Hansen typology has the advantage in allowing for measurement of process innovation without being confounded with the technology that facilitates the process innovation.

An additional dimension of innovation has to do with the impact of the innovation on the industry. As described by Betz, discontinuous innovations result in creating or altering industrial structures; continuous innovations reinforce the existing structure [73]. The essence is the degree of 'radicalness' of the innovation. Thus, discontinuous innovations are also referred to as radical, next-generation, revolutionary, breakthrough, or disruptive. Continuous innovations are also referred to as sustaining, evolutionary, or incremental. Johannessen et al. viewed this dimension of innovation from the viewpoint of newness but specifically 'new to 
whom?'; in their view, incremental innovations are new to the adopting firm whereas radical innovations are new to the industry [76].

Cooper (citing Utterback [77]) defines a multidimensional model of innovation that presents a graphical depiction of the preceding discussion on innovation typology. The model is shown in Figure 4.

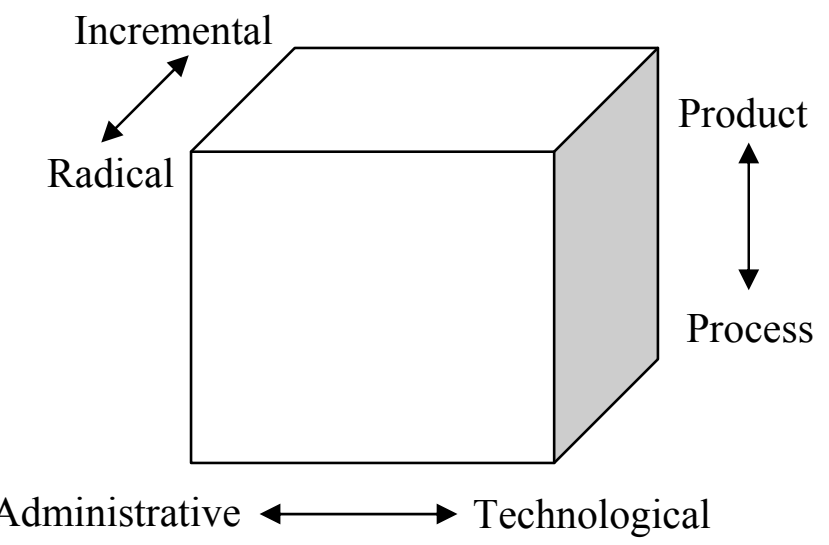

Figure 4. A Multidimensional Model of Innovation [72]

In this '3-D' view of innovation, any individual innovation can involve varying degrees of each of the three dimensions. Hence, overlap of different forms of innovation, specifically process and technological innovation as discussed previously, is readily acknowledged in this model. The author cites the use of the Internet to advertise and distribute products/ services as a type of administrative-product innovation because it involves altering the product/ service offered as well as organizational procedures and structures. However, for future research, the author poses questions regarding the relative importance of each type of innovation and 
implications for organizational structure and strategy. For example, if the degree of radicalness of an innovation overshadows the other dimensions and centralization is shown to foster adoption of radical innovations, does that outweigh the importance of decentralization to foster adoption of process innovations? Although this question is merely posed as a suggestion for future research, there are implications for measuring organizational innovation performance. Specifically, measurement instruments that define innovation performance narrowly may either fail to account for the contingency approaches firms take in pursuing innovation and/ or implicitly predetermine the relative importance of the various forms of innovation.

In addition to the multi-dimensional nature of innovation, Damanpour's definition alludes to the possibility of varying definitions based on one's perspective. Rogers makes this explicit in stating that innovations are something “...perceived as new by an individual or other unit..." Therefore, by this definition, innovation may be measured via subjective as well as objective measures.

As discussed previously, innovation research has also emphasized creation as well as adoption of innovations. Authors such as Betz have focused primarily on innovation from the standpoint of creating something new [73]. Viewed this way, innovation performance would be measured via metrics such as number of patents and new products introduced. Other authors have focused on the process by which firms adopt or implement something new [74, 75]. Damanpour further decomposes innovation adoption into the stages of initiation (problem perception, information 
gathering, attitude formation and evaluation, and resource attainment leading to a decision to adopt) and implementation (events and actions pertaining to modification of both the innovation and the organization, the initial utilization, and the continued use of the innovation until it becomes a routine feature of the organization) [74]. From the standpoint of adoption, innovation performance would be measured via metrics such as new processes implemented, new sources of supply, and new ways of organizing.

Given the preceding discussion of the varied dimensions and definitions of innovation, one can see there are close parallels with quality. Both quality and innovation are inherently multi-dimensional and can be measured objectively as well as subjectively. However, prior to turning attention to the literature on measurement of innovation performance, further development of innovation theory, particularly with respect to relationships to quality management will be discussed.

Innovation Theory Development

Utterback and Abernathy explored relationships between innovation and competitive strategy and between innovation and production process characteristics [78]. The authors propose models for both process and product development and integrate these models into three stages:

Stage I - processes are uncoordinated, rates of change are high and processes are not yet standardized; processes are organic and respond easily to environmental change. Performance-maximizing is emphasized early in the 
product life cycle when product change is rapid; firms rely on external sources of information as innovations in this phase are product-focused and stimulated by the market; the primary insight for innovation is in product requirements; "the locus of innovation is in the individual or organization that is intimately familiar with needs"; priority given to innovation as a competitive strategy is greatest in this stage; innovations in this stage are 'original' (or created vs. adopted to use the terminology presented above).

Stage II - processes are segmental, price competition becomes more intense; production systems become more mechanistic and are designed for efficiency; there is greater use of automation and process control, however some processes are still manual (hence the term 'segmental'); process innovation tends to be highest in this stage. Sales-maximizing is emphasized as market uncertainty lessens and some product designs begin to dominate; firms define needs based on their visibility to the customer; greater use of advanced technology is possible for product innovation; process innovation (which may be radical) is stimulated by the need for increased output.

Stage III - processes are systemic and so well integrated that change is more costly; process redesign is slower and may be spurred by new technology or shift in market requirements; resistance to change can lead to either economic decay or revolutionary change; firms that pursue high productivity to the extreme may find they have achieved this gain at the cost of lost flexibility and innovative 
capacity. Cost-minimizing is emphasized as the product life cycle matures, products become standardized, and competition shifts to price; production focuses on efficiency and economies of scale; significant change is costly (benefits are often marginal), and requires a systems focus for both product and process; innovations in this phase are typically incremental and developed by someone that brings new technological insights to the problem such as the R\&D group or an equipment supplier.

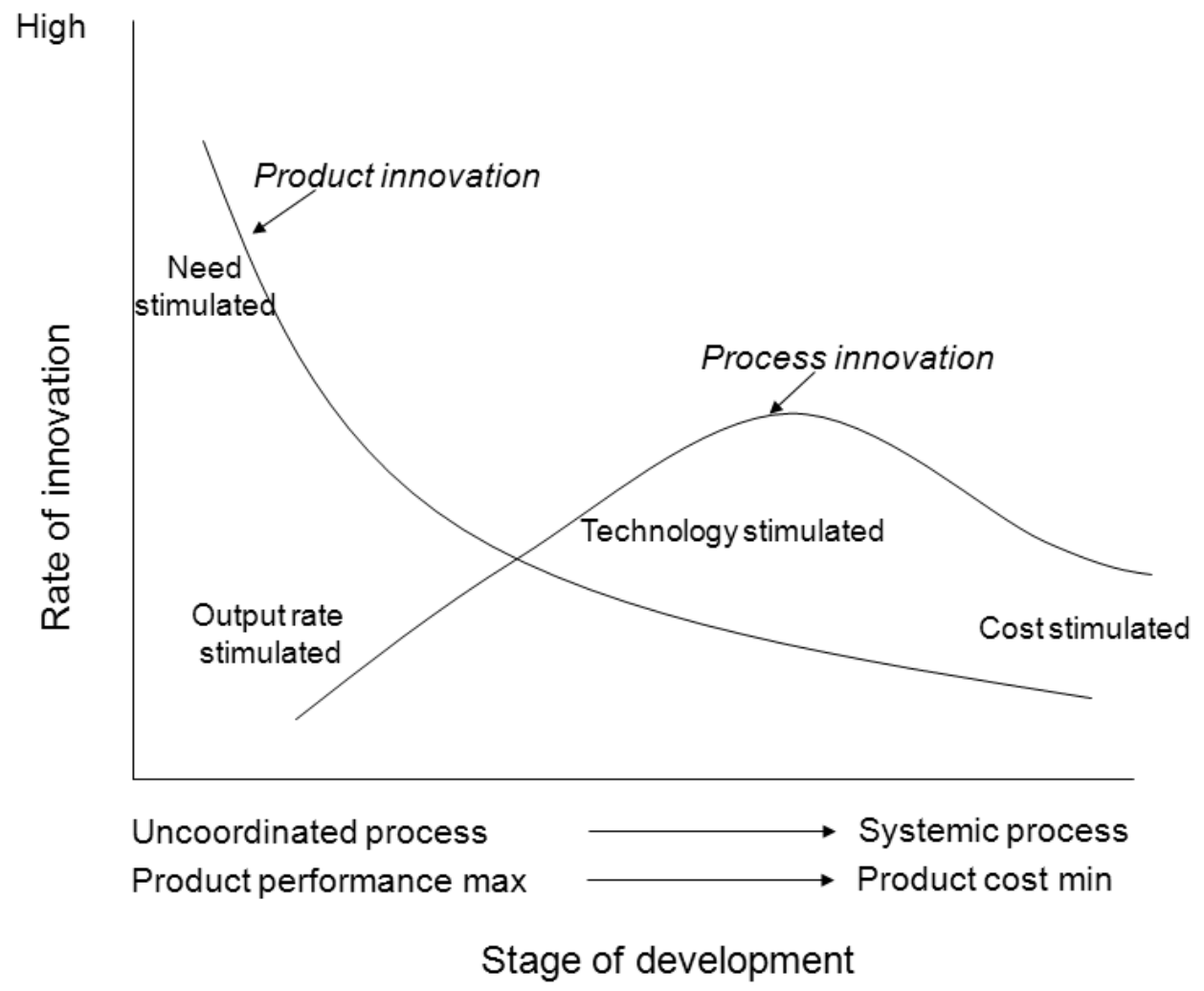

Figure 5. Innovation and Stage of Development [78]

Figure 5 summarizes the product and process development models, how the models vary with stage of product and process development, the differing emphases placed on product and process innovation, and the changing locus of innovation. 
Key points from this paper from the standpoint of measurement of innovation include reinforcement of the multidimensional nature of innovation. For example, few if any Stage III firms would be deemed innovative if innovation was defined as radical, original (created), and product-focused. Conversely, if innovation was more broadly defined and included incremental, adopted, and process-focused innovations, the results would likely be quite different.

From the standpoint of quality management, Utterback and Abernathy draw linkages to the importance of customer relationship management and external communication. Specifically, given that the locus of innovation in Stage I is in firms intimately familiar with the needs of the market, it is crucial for such firms to take a proactive approach to customer focus. Further, the authors note the importance of understanding the locus of innovation and the difference between creation and adoption of innovation. In this regard, assessing the innovation performance of an industry sector in Stage III, for example, lumber manufacturing, would best be measured from the viewpoint of adoption of process innovations. At the same time, assessing the innovation performance of equipment suppliers to lumber manufacturers could involve a broader view of innovation.

Damanpour conducted a meta-analysis of innovation to identify relationships between innovation and organizational determinants such as specialization, functional differentiation, centralization, and internal/ external communication [79]. The author 
synthesized results of published empirical research to provide information for theory development and to address existing questions on innovation.

From the standpoint of quality management, key findings from this research include the finding of positive associations between innovation and internal and external communication. Again, external communication lends support to the idea that customer relationship management leads to innovation. Further, given that 'information and communication systems' are a key factor in TQM, there is a potential link to the importance of internal communication as well.

In addition, Damanpour states that standardization of work processes, which is often emphasized in the TQM construct for process management, can establish the managerial control required for successful adoption of innovation. This finding may beg the question as to whether TQM practices are more closely connected to adoption of innovation rather than creation.

Type of innovation (which Damanpour defines broadly as product/ process; administrative/ technical; and radical/ incremental) did not influence the relationships between organizational determinants and innovation. This finding seems to contradict the suggestion by Cooper of the importance of a multidimensional view of innovation [72]. However the finding is supported by Johannessen et al. (described below) in that their research suggests that measuring innovation via a single construct is possible [76]. 
Damanpour also found that direct supervision inhibits innovation, lending support to the employee empowerment aspect of TQM. With respect to organizational culture, the author found that an organic culture enhances initiation of technical innovations whereas a mechanistic culture supports implementation of administrative innovations.

Damanpour also examined the relationships between organizational complexity and innovation [74]. Key findings from this research from the standpoint of quality management include a positive relation between innovation and organizations facing high environmental uncertainty. This finding agrees with the 'control vs. learning' dichotomy within quality management expressed by Sitkin and described below [24]. Also, in defining product and process innovations, the author presents a possible distinction between two types of innovation and internal vs. external focus. Specifically, product innovations “... refer to the introduction of new products or services to meet an external market or user need", while process innovations refer to the “....introduction into the organization's production process or service operations of new elements (e.g., input materials, task specifications, work and information flow, and equipment) that are used to produce a product or render a service." Hence, there may be a tendency towards product innovations in firms that are externally focused and process innovation in firms that are more internally focused. 
In summary, the dimensions (product/ process, adopt/ create, and radicalness) and locus of innovation change in an organization depending on stage of the product life cycle and degree of development of the firm's processes. In the early stage of a firm, the emphasis is on the creation of innovative products with customer needs serving as a crucial source of information. Later in a firm's development, the rate of innovation is much lower and the emphasis is typically on adoption of process innovation provided via a vendor. These concepts reinforce the notion of the multidimensional nature of innovation and the imperative to take this into account when measuring organizational innovation performance. Further, there are a number of areas that point to connections between innovation and key aspects of quality management. For example, internal (e.g., process focus/ continuous improvement) focus may lead to an emphasis on process innovation whereas external (customer) focus may lead to product innovation. Prior to exploring additional conceptual linkages between innovation and quality, and between quality management and innovation performance, the means by which innovation performance is measured must first be addressed.

\section{Measuring Innovation Performance}

While there has been extensive research in the quality field related to the development of measurement constructs for assessing the extent of implementation of TQM, constructs for innovation are not as well developed [25]. For purposes of discussion here, the primary constructs of interest are innovation outputs, that is, metrics that effectively reflect innovation performance. Common measures of 
innovation performance include patents and/ or new product releases [80]. However, these metrics represent a fairly narrow perception of innovation.

As stated above, Johannessen et al. proposed that 'newness' was the common denominator for the various definitions of innovation [76]. The concept of 'new to whom?' is presented in the context of radical vs. incremental innovations; the authors state that the relevant unit of adoption must be addressed to fully understand if an innovation is new to the firm or new to the market. In other words, an incremental innovation is one that is new to the firm, but not new to other firms. Conversely, radical innovations are new to the market. In essence, the more broadly the unit of adoption is defined, the more radical the innovation.

The authors state that earlier studies focused on a narrow view of innovation and often used proxies such as total R\&D expenditures, proportion of $R \& D$ scientists and engineers and number of patents rather than explicitly measuring what is new. Based on a review of the innovation literature, six forms of innovation are proposed: new products, new services, new methods of production, opening new markets, new sources of supply, and new ways of organizing. A measurement instrument was developed and tested (see Appendix B1) based on these six forms of innovation. Factor analysis showed that innovation could be measured as a single construct and distinguished only by the degree of radicalness. Reliability was deemed to be adequate given that Cronbach's alpha was greater than the 0.70 threshold suggested by Nunnally [69]. Hence, this result calls into question the need to measure innovation 
via multiple dimensions - at least from the standpoint of product vs. process and administrative vs. technical.

However, while it may be possible to measure innovation via a single construct, researchers must determine if it is appropriate or desirable given the specific objectives of the research at-hand. For example, if studying relationships between management practices and innovation in general terms, it may be sufficient to simply conceive of innovation as a single construct. However, if the objective is to examine the correlations between specific practices and different types of innovation, a single construct for innovation would make this impossible.

The authors also discuss important external factors influencing innovation that include customer-supplier relations and internal factors such as information and communication. Further research is suggested to consider how innovation is related to internal and external factors.

Strengths of the measurement instrument developed by Johannessen et al. include that it is both simple and comprehensive. With regards to simplicity, response rate might be better in that respondents would not be overwhelmed by the number of questions. Comprehensiveness is evident in that the dimensions of product, process and business system are all addressed to some extent. At the same time, one might question as to whether the instrument is too simple, i.e., is a single question on product innovation sufficient to capture the many nuances of this form of innovation? In that 
same regard, is the instrument truly comprehensive with only six questions to cover the many facets of innovation?

Diaz-Balteiro et al. explored productive efficiency and innovation activity in the Spanish wood products industry [81]. Innovation output was measured as number of patents, number of product innovations and number of process innovations. Product innovation included “...introduction of new and significantly improved goods and/or services with respect to their fundamental characteristics, technical specifications, incorporated software or other immaterial components, intended uses, or user friendliness." Process innovation was a variable equal to two, one, or zero two if the firm adopted both types of process innovation (introduction of new and significantly improved production technologies or new and significantly improved organizational and managerial changes); one if the firm adopted one of the two types of process innovation; and zero if the firm did not adopt any process innovation. The inclusion of 'organizational and managerial changes' indicates these authors view administrative/ business system innovations as a type of process innovation. The article does not mention testing the constructs for reliability. As the authors used DEA as their research method, this article will be addressed in greater detail in the appropriate section below.

Prajogo and Sohal $[25,30,35,82]$ developed innovation performance metrics to explore the correlation between quality management and innovation performance. Constructs for measuring product and process innovation were developed based on a 
review of the literature discussing empirical studies of innovation. Reliability of the constructs was found to be adequate in that Cronbach's alpha was greater than the 0.70 threshold suggested by Nunnally [69]. The criteria developed are shown in Table 2 below.

Table 2. Scales to Measure Product and Process Innovation Performance [25]

\begin{tabular}{|c|c|}
\hline Product innovation & Process innovation \\
\hline $\begin{array}{l}\text { The level of newness (novelty) of } \\
\text { new products } \\
\text { - The use of latest technological } \\
\text { innovations in new product } \\
\text { development } \\
\text { - The speed of new product } \\
\text { development } \\
\text { - The number of new products } \\
\text { introduced to the market } \\
\text { - The number of new products that are } \\
\text { first-to-market (early market } \\
\text { entrants) }\end{array}$ & $\begin{array}{l}\text { - The technological competitiveness } \\
\text { - The updated-ness or novelty of } \\
\text { technology used in processes } \\
\text { - The speed of adoption of the latest } \\
\text { technological innovations in } \\
\text { processes } \\
\text { The rate of change in processes, } \\
\text { techniques and technology }\end{array}$ \\
\hline
\end{tabular}

These scales were used to acquire perceptual data from survey respondents.

Personnel in manufacturing and non-manufacturing firms were asked to evaluate their company's performance with respect to each of the items in Table 2 against the major competitor in the industry. Several of these questions (e.g., level of newness and firstto-market) were included to specifically address radical forms of innovation. A strength of the instrument is measuring both product and process innovation via multiple perspectives, for example number of products, speed of new product development, and level of newness. This is a strength in comparison to the Johannessen et al. instrument described above. At the same time, a limitation is that the instrument does not address administrative or business systems innovations. As the authors used the instrument to assess the impact of TQM on quality and innovation 
performance, the article will be discussed in greater detail in the appropriate section below.

Singh and Smith developed four measures for innovation [33]:

- innovative processes/products/services have been commercialized;

- R\&D leads to development of world-class techniques/technologies;

- the rate of innovation of new operational processes; and

- the rate of introduction of new products and services.

As these authors used these measures in a study exploring the relationships between TQM and innovation, the paper will be explored in more detail in the appropriate section below.

Thus, in comparison to quality performance, there are fewer instruments to choose from to evaluate innovation performance. However, two of the instruments described above (Johannessen et al. and Prajogo and Sohal) have been found to be reliable instruments for measuring innovation performance. To measure innovation performance from multiple dimensions (e.g., product/ process/ business system and radical/ incremental) and multiple perspectives (e.g., number of products developed and speed of product development), it seems that some combination of the two instruments is needed.

The review of the literature to this point has emphasized the varying definitions, dimensions, and measurement instruments for determining the extent of implementation of TQM (i.e., the inputs an organization invests into their quality 
management system) and quality and innovation performance (i.e., outputs). To determine the 'benchmark' organizations - those that are most efficient at converting inputs into outputs - an analytical tool is needed that can account for multiple inputs and outputs simultaneously, and further, for systems for which no pre-defined production function exists. Data Envelopment Analysis (DEA) is an approach that fulfills these requirements. And further, to enable detailed descriptions of best practices, the results of DEA analysis can be used for identification of target organizations for case study research. In this regard overviews of DEA and the case study method will be presented prior to a discussion of the literature describing relationships between quality management and organizational performance.

\section{Data Envelopment Analysis (DEA)}

Presented here is an introduction to Data Envelopment Analysis (DEA). The focus is on describing DEA, how it works, and to describe its applicability and advantages for exploring the impact of quality management on performance. Applications of DEA to efficiency measurement in quality management and innovation will be discussed in a separate section.

Charnes, Cooper, and Rhodes [83] authored the seminal paper on Data Envelopment Analysis (DEA) in 1978. DEA provides a measure of efficiency via simultaneous comparison of multiple inputs and multiple outputs. This issue of multiple inputs and outputs is a key advantage of DEA. Efficiency is a wellestablished concept of the ratio of one output measure (such as profit) to one input 
measure (such as total labor). However, in situations where there are multiple inputs and multiple outputs with no production function to describe the relationships between values, there can be an overwhelming number of efficiency ratios. In short, it becomes exceedingly difficult to determine efficiency of a system as a whole. Such is the case for the topics of discussion here - quality and innovation - that are represented by multiple inputs and outputs with no clear production function describing the relationship.

For purposes of discussion here, the various principles and practices of TQM can be seen as the inputs. It would seem obvious that the primary desired output of a quality management system is quality performance. However, as will be discussed below, research has shown the fundamental principles of TQM (process focus/ continuous improvement, customer focus, and participation and teamwork) also impact innovation performance. Thus, both quality and innovation performance are reasonable outputs for TQM. At the same time, such efficiency analyses could benefit from also taking into account the practices organizations invest in with the specific intent of achieving innovation performance, i.e., practices related to R\&D and technology management.

DEA uses linear programming (LP) to determine the relative efficiency of decision making units (DMUs, e.g., companies, departments, etc.). Unlike regression analysis, DEA is an extreme point method. The goal is to identify those DMUs that 'set the benchmark' by demonstrating, with actual production and performance data, 
the maximum production of outputs given inputs (what is termed output-oriented in DEA), or conversely, the minimum usage of inputs, given outputs (input-oriented).

Analysis of productive efficiency via DEA provides both an efficiency score as well as data to enable inefficient DMUs (those that could obtain more output with the same input or vice-versa, the same output could be obtained with less input) to determine which aspects of the business require attention to improve efficiency. Thus, DEA serves a dual role as an analytical tool for benchmarking as well as a tool to enable decision makers to determine leverage points for improvement.

There are numerous models by which DEA analyses are implemented as well as extensions to the traditional approaches. For example, models may be either inputor output-oriented as described above, may emphasize constant or varying returns to scale, and may consider the slack values in the LP such that 'weakly efficient' DMUs ${ }^{2}$ may be identified (see the figure in Appendix C). One such model is CCR (Charnes, Cooper, and Rhodes model) [83] shown in ratio form in Equation 1:

\footnotetext{
${ }^{2}$ Weakly efficient DMUs have non-zero slack whereas the slack values are zero for 'strongly efficient' DMUs. In other words, a weakly efficient DMU is one that is on the efficiency frontier, however from an output perspective, other DMUs exist with the same level of inputs, but more outputs. See the figure in Appendix $\mathrm{C}$ for a graphical view of the concept.
} 
$\max z=\frac{\sum_{r=1}^{s} \mu_{r} y_{r o}}{\sum_{i=1}^{m} v_{i} x_{i o}}$

$$
\begin{gathered}
\text { s.t. } \frac{\sum_{r=1}^{s} \mu_{r} y_{r j}}{\sum_{i=1}^{m} v_{i} x_{i j}} \leq 1 \quad j=1, \ldots, n \\
\mu_{r}, v_{i} \geq 0 ; r=1, \ldots, s ; i=1, \ldots, m
\end{gathered}
$$

Where:

$z$ is the efficiency score;

$x$ values are inputs;

$y$ values are outputs;

$i$ is an index for inputs and $m$ is the number of inputs;

$r$ is an index for outputs and $s$ is the number of outputs;

$j$ is an index for DMUs and $n$ is the number of DMUs;

$\mu$ is the weight applied to inputs

$v$ is the weight applied to outputs

$o$ refers to which of the n DMUs is being analyzed in this particular iteration

Hence, each DMU's efficiency score $(z)$ is the ratio of weighted outputs to weighted inputs; as with 'traditional' measures of efficiency, the objective is to maximize this ratio. Each DMU is able to select its own set of weights for inputs and outputs that provides the maximum ratio (efficiency score) possible. However the equation constrains each DMU such that this ratio must be less than or equal to 1 . Thus, the weights chosen by an individual DMU cannot result in a score greater than one when applied to the inputs and outputs of any of its peers. In short, the approach is intended to allow the 'truly exceptional' (i.e., benchmark) DMUs to shine. 
Charnes et al. adapted the ratio model to allow for solution as a set of linear programs. The equation, adapted to reflect an output-orientation is as shown in Equation 2:

$$
\begin{aligned}
& \min q=\sum_{i=1}^{m} v_{i} x_{i o} \\
& \text { s.t. } \sum_{i=1}^{m} v_{i} x_{i j}-\sum_{r=1}^{s} \mu_{r} y_{r j} \geq 0 \quad j=1, \ldots, n ; j \neq o \\
& \sum_{r=1}^{s} \mu_{r} y_{r j}=1 \\
& \mu_{r}, v_{i} \geq 0 ; \forall r, i
\end{aligned}
$$

Where:

$\mathrm{q}$ is the output-oriented efficiency score; Other variables are as in Equation 1.

The adaptation to output-orientation results in the objective of minimizing, rather than maximizing, the efficiency score. Efficient firms are those achieving the maximum outputs given inputs; conversely, inefficient firms are those for which outputs could be increased while holding inputs constant.

The complex, multidimensional nature of both quality and innovation lead to challenges in using traditional approaches to simultaneously examine relationships between multiple inputs and multiple outputs. Further, as has been discussed at length, there is no one 'standard' approach to implementing TQM. The key principles underlying TQM must be implemented using a contingency approach dependent on 
the firm's strategy, objectives, and context. DEA allows for individual DMUs to select the best 'mix' of inputs to achieve a given level of outputs. This is quite appropriate for TQM given the mix of hard and soft factors, internal and external focus, etc. DEA will enable the exploration of how TQM is currently being implemented in actual firms in light of the firm's performance.

Lastly, the principle of benchmarking within TQM is in direct agreement with DEA given that DEA is a key tool for identifying benchmark firms and benchmark targets. In fact, there is precedence in the literature for such alignment. The two studies the author could locate on application of DEA to examine TQM practices include a study by Forker et al. and Yoo. Forker et al. used DEA to evaluate the TQM practices of supplier firms as a means of benchmarking best peer suppliers [84]; Yoo used DEA to examine the impact of specific TQM principles and practices on quality performance [85]. Each of these studies is examined below in the section related to 'Relationships between Quality and Performance - Studies using DEA.'

In summary, DEA is well suited to the identification of best quality management practices for achieving quality and innovation performance. The method allows for simultaneous consideration of the multiple quality management inputs and the multiple outputs inherent to both quality and innovation performance. With respect to examining TQM practices, DEA would enable identification of firms that have focused on specific practices rather than those taking more of a 'shotgun' approach (i.e., emphasizing all practices). The latter firms would be excluded from 
the efficient set to the extent that competitors are able to achieve similar or better results by emphasizing fewer practices (and presumably investing fewer resources). In short, as a benchmarking tool, DEA is ideal for identifying 'best practice' firms those that are focused and achieving results. However, more in-depth analysis is needed to provide detailed descriptions, and specific examples, of these practices. In this regard, the case study method is well-suited for use as a follow-up to DEA.

\section{Case Study Method}

Eisenhardt defines the case study method as a research strategy which “...focuses on understanding the dynamics present within single settings" [86]. Similarly, Yin states that case study research focuses on investigation of contemporary phenomena in their real-life context [87]. Hence, setting or context is crucial in case study research; the strength of the method lies in obtaining detailed data about a timely topic in the context in which the topic occurs.

There are multiple potential sources of data for case studies; information can be acquired from sources including archives, interviews, observations, or simulations [88]. Case study data can be both qualitative and quantitative, although, qualitative data are widely used. Given the importance of context discussed above, data are typically collected in the field, i.e., from visits to case organizations. 
Glaser and Strauss state that the case study method (or grounded theory - an outcome of case study research) is useful in areas that are not yet well-explored, i.e., where theory is not yet well developed [89]. Further, case studies are likely to provide well-grounded and detailed descriptions of the situation being studied [86]. Yin describes several situations where case studies are applied [88]. One such application is to explain causal links in situations that are too complex for other research methods such as surveys or designed experiments.

Many authors have proposed a step-by-step approach to case study research, although the process is typically iterative. Eisenhardt [86], for example, describes in some detail eight steps involved in case study research. These steps may be summarized as follows:

1. Getting started - describe the factors and guiding propositions (expected relationships) among them and propose research questions.

2. Selecting cases - 'theoretical sampling', rather than random sampling is preferred - cases should be selected to replicate previous cases, extend emergent theory, represent theoretical categories, or polar opposites; multiple cases are preferred to provide deeper understanding and the chance to test propositions.

3. Crafting instruments and protocols - data are typically gathered using a number of different methods including quantitative as well as qualitative and through using multiple investigators where possible.

4. Entering the field - researchers visit case firms to gather data; detailed recordkeeping is crucial in this step; analysis should occur simultaneously with data collection via addressing questions such as "what am I learning" or "how does this case differ from the last"; further, a key hallmark of the case method is in the flexibility - the researcher is encouraged to make adjustments to measurement instruments and methods during data collection to explore emerging themes or take advantage of special opportunities. 
5. Analyzing data - triangulation is conducted to compare data collected by various researchers and methods (e.g., interview, archive, etc.) to discover the extent to which there is convergence among different sources; data are analyzed both within-case as well as cross-case:

a. Within-case - develop detailed case study reports for each case; code and tabulate data.

b. Cross-case - select categories or dimensions, pairs of cases, and/or varying data sources and look for similarities and differences between them; the 'chain of evidence' is crucial in being able to document links between questions asked, data collected, analysis, and conclusions.

6. Shaping propositions - again, the process is iterative - initial propositions are compared with data and new propositions often emerge; the process of proposition formation and data collection and analysis continues until there is a close fit with the data; each case serves as replication in that each case may confirm or disconfirm the proposition; these processes sharpen the definition, validity, and measurability of the constructs and build internal validity.

7. Enfolding literature - given the typically small number of cases, comparison of results with existing literature (both similar and conflicting) is done to ensure reliability and validity of findings; generalizability of results occurs when findings are confirmed by a broad range of literature on the topic.

8. Reaching closure - the researcher must determine when to stop adding cases (although number of cases is usually predetermined and four to ten cases usually work well) and when to stop iterating between data collection and theory development; the 'stop point' is usually when theoretical saturation occurs, i.e., when incremental improvement to the theory is minimal.

Case study research has several advantages including applicability to new areas where theory development is in the early stages and in tightly linking theory with real-world data. Further, the method allows for in-depth analyses and detailed descriptions of the underlying reasons behind the phenomena being examined. Also, the process is flexible and allows for 'real time adjustments' to the method to explore concepts revealed during the study. 
However the approach is not without disadvantages as well. There is often an enormous amount of data collected which can result in development of an overly complex theory in an effort to incorporate all the information. Field work has risks in the researcher being 'too close to the situation', that is, allowing the situation to guide data collection rather than the research objectives. There are also risks from respondent bias in that responses from personnel with higher status in an organization might receive higher weight or credibility than those of lower status (regardless of the accuracy of the comments). Similarly, there are risks of respondents 'guarding' or inaccurately relaying sensitive information. As has often been said, the most interesting information is often the most difficult to obtain. Finally, and significantly, methods for analyzing qualitative data are not yet well established and thus the findings often do not carry the weight of statistical evidence reported using more traditional, quantitative approaches.

Perhaps one of the greatest challenges with case research is related to case selection, that is, how the researcher goes about selecting the individual cases to be explored [90]. There has not been a great deal of attention given to this topic in the literature. For example, Yin devotes only one page (p. 78) to the topic in his text $\underline{\text { Case }}$

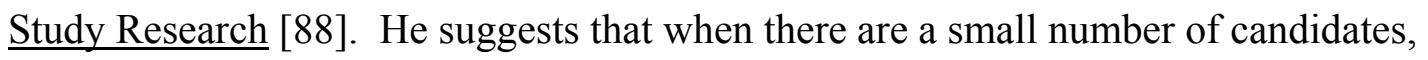
the researcher may screen candidates by querying people knowledgeable about each candidate and/or collecting limited documentation about each candidate. Researchers are encouraged to, “...have defined a set of operational criteria whereby candidates will be deemed qualified to serve as cases. Then you should select randomly from the 
qualified candidates..." If the set of candidates is larger, Yin suggests that a two-stage screening procedure may be needed. The first stage consists of collecting relevant quantitative data about the entire pool from some archival source to screen the group down to 20 to 30 candidates; stage two is the same as described previously for smaller groups.

Eisenhardt also addresses case selection [86]. As stated above in step 2, 'theoretical sampling', rather than random sampling is preferred. That is, cases are chosen for theoretical not statistical reasons. As she states, cases should be selected to replicate previous cases, extend emergent theory, represent theoretical categories, or polar opposites. She then provides several examples of how other researchers have selected case firms. For example, one researcher wanting to develop theories on success and failure selected four markets and then polar examples from each market, i.e., "...one case of clearly successful firm performance and one unsuccessful case." Such an approach may work in situations where 'successful' and 'unsuccessful' are in fact clear. The challenge is in situations where such distinctions are not clear; even as with Yin's suggestions, when a priori data on candidate firms are not readily available.

Seawright and Gerring propose seven quantitative methods for case selection [90]. As these authors state, “...choosing good cases for extremely small samples is a challenging endeavor... the question of case selection has received relatively little attention from scholars..." The proposed methods include several approaches for 
choosing cases based on a set of independent variables (X) and a dependent variable (Y):

- Typical - cases are typical examples (representative) of some relationship; identified via looking for the smallest possible residual for all cases in a multivariate analysis

- Diverse - cases exemplify diverse values of some measure of interest; identified via standard deviations of X or Y, cross tabulations, factor analysis, or discriminant analysis

- Extreme - extreme or unusual values of some measure of interest; identified via absolute value of $\mathrm{z}$ score, i.e., a value that is many standard deviation's away from the mean

- Deviant - cases deviate from some relationship; identified via maximum deviation from regression line

- Influential - cases with influential configurations of the independent variables; identified via regression analysis using Cook's distance (measure of the extent to which estimates of parameters would change if a given case were omitted from the analysis)

- Most similar - cases are similar on some specified variable; identified via 'matching strategy/technique' (statistical method) - identify set of variables on which cases are to be matched, search for cases with identical scores on covariates (from regression)

- Most different - cases are different on some specified variable; corollary to most similar method

Common to all of these methods is the need for quantitative data (e.g., archival data or from surveys) from the candidate firms. Also common to the methods is the requirement for a single dependent variable. Therefore, in situations where there are multiple outputs (dependent variables) of interest, none of the methods proposed by Seawright and Gerring are appropriate. Such is the case here with regards to quality and innovation given that both are measured via multiple dimensions. Therefore, the 
need remains for a method to select the case firms in situations with multiple inputs and outputs.

An analog to Seawright and Gerring's approach for 'diverse' case firm selection would be to use cluster analysis on either inputs or outputs to aggregate cases on some variable of interest and thereby facilitate case selection. Such an approach was used by Campbell and Ahrens to select case firms [91]. The authors used cluster analysis to organize communities into three groups with respect to the support the communities provided for victims of rape. Case firms were then selected from the high and low extremes of support. Such an approach appears to be the best available option for situations where the criteria for case selection are multidimensional.

In summary, case study research is applicable to exploring timely topics in the context of the case firm. The method is often used for areas where the underlying theory is not yet well-established. The results can be novel, and rich descriptions of underlying causes and relationships for situations too complex for the use of other research methods. The process of conducting case study research is iterative where data collection, analysis, and theory development occur simultaneously. Case firms are chosen by theoretical, rather than random sampling, and polar opposites with regards to a specific phenomenon can be selected to provide a diversity of examples.

The combination of DEA, cluster analysis, and case studies provides distinct advantages for identification of best practices. DEA can be used to identify firms that are efficient with respect to some multidimensional performance measure of interest 
(e.g., quality and innovation performance); cluster analysis can be used to group firms with respect to these measures of performance to thereby facilitate case firm selection. Case studies can be then used to explore in greater depth the key practices that differentiate firms, verify results, and add deeper insights and detail to the best practices.

To this point, the review of the literature has focused on the varying definitions, dimensions, and measurement instruments for determining the extent of implementation of TQM and quality and innovation performance. DEA followed by cluster analysis was presented as a method to determine the 'benchmark' organizations - those that are most efficient at converting quality management inputs into either quality or quality and innovation performance outputs. And finally, the case study method was presented as an approach to confirm and add depth to the best practices. The review of the literature continues with an analysis of studies that have examined relationships between quality management and organizational performance.

\section{$\underline{\text { Relationships between Quality Management and Organizational Performance }}$}

As discussed previously, the development of instruments to evaluate the extent of implementation of TQM principles and practices was inspired in part to respond to conflicting accounts of TQM to generate significant positive impacts on organizational performance. Following the development of such instruments, researchers were better able to assess correlations between firm performance and the extent to which TQM 
was implemented in the firm. Relationships between TQM and general organizational performance (including quality) using a variety of research methods have been explored via various approaches, including a limited number of studies using DEA. More recently, relationships between quality management and innovation performance have been explored. The literature in each of these areas will be explored in turn.

\section{General Organizational performance}

As stated in the introduction, results of studies exploring the impact of TQM on performance have been mixed. While the literature on this topic within the last 10 to 15 years is vast, some of the key studies are presented here.

Garvin conducted one of the earliest comprehensive studies to address the question of the impact of quality on corporate performance [57]. The study was limited to an examination of product quality as opposed to the much broader conception of TQM. Regardless, the results paved the way for other research exploring this critical connection. Garvin examined quality and productivity for air conditioner manufacturers in the U.S. and Japan. Quality was measured as the incidence of internal failures (defects observed during fabrication or in assembly) and external failures (i.e., field failures, measured as service calls recorded during the first year of warranty coverage). The results showed that the failure rate for the worst Japanese company was less than half that of the best U.S. manufacturer. Total costs of quality incurred by Japanese producers were less than half the failure costs incurred by the best U.S. companies. Highest quality producers also had the highest productivity 
(output per man-hour). The conclusion is thus that quality adds to the bottom line by significantly reducing failure costs and increasing productivity (quality professionals have often had to argue the case that productivity must be defined as good output divided by total input and not simply total output divided by input [92]).

Adam studied the correlation between quality improvement approaches and operating and financial performance [4]. The correlation between quality improvement and productivity improvement approaches and performance quality (defined as average percent items defective, cost of quality as percent of sales, and customer satisfaction) was strong whereas the relationship between these approaches and operating or financial performance was significant, but weaker. The authors state that given the low $\mathrm{R}^{2}$ values, “...TQM and other commonly promoted practices have little practical influence on the performance variables reported in this study... A case can be made from this study that TQM is a failure." The author suggests a contingency approach to quality and productivity based on the strategic objectives of performance quality, operating improvement, or financial performance. Other authors have suggested that the absence of a strong relationship in this research was due to an inappropriate model used for examining the quality management - quality performance relationship [38].

Flynn et al. used the quality management measurement instrument they developed (discussed above and shown in Appendix A2) to link specific practices for high- and low-quality firms [93]. Quality performance was defined as the percentage 
of products that pass final inspection without requiring rework. The authors acknowledge this narrow definition as a significant limitation for the research and suggest future research to examine a broader definition of quality performance measures including measures taken from the customer viewpoint. High-quality firms (i.e., those with a high percentage of products that pass final inspection) could be differentiated from low-quality firms based on employee involvement, process control, new-product quality practices, concurrent engineering, feedback, maintenance, supplier relationship, labor skill level and selection for teamwork potential. Customer interaction and design characteristics did a poor job of differentiation. One surprising result was that both high- and low-quality groups used more progressive quality management practices (i.e., those measured in the study) while the intermediate group used less progressive practices (or simply used the same practices to a much lesser degree). The authors hypothesized that this unexpected outcome may be due to lowquality firms just beginning to adopt a broader set of quality practices and not yet reaping the results. The intermediate firms may simply be content with the status quo of quality in their firm and thus continue to use outdated practices.

Forker et al. examined how each of the functional areas of a firm addresses the different dimensions of quality and which dimensions are important for organizational performance [39]. The authors address one of the key limitations of prior research examining the quality-performance relationship, namely the definition of quality as conformance to specifications. This definition of quality is but one of Garvin's five definitions of quality [55]. Forker et al. examined quality using Garvin's eight 
dimensions: conformance to specifications, product reliability, product durability, design quality, product improvement, brand image, company reputation, and customer service [55]. Interview respondents in the furniture industry were asked to rank the strategic importance of each of these eight dimensions. Respondents were also asked to indicate percent responsibility of manufacturing, marketing, and product design \& development for each dimension. Organizational performance was measured as return on assets (ROA), return on investment (ROI), growth in ROI, sales growth, market share, growth in market share, return on sales (ROS), and growth in return on sales. These values were measured both subjectively (the firm's perception of performance relative to major competitors on a seven-point scale) and objectively (actual data for companies willing to provide the information). Only about one third of respondents were willing to provide the financial measures. However, subjective and objective measures were sufficiently correlated such that subjective measures could be used for the analyses. Simple regression analysis followed by stepwise regression was used to examine the contribution of the different quality dimensions to performance. Results showed that quality viewed as conformance to specifications is significantly related to ROI growth, sales growth, and ROS growth. Reliability is related to ROA and durability is related to ROA, ROI growth, and ROS. Design quality and product improvement are the most consistent predictors of overall organizational performance. This is an intriguing result in that these activities would traditionally be viewed as more closely related to product innovation than quality management. Stepwise regression revealed that design quality, product improvement, company reputation, product reliability and conformance to specifications are all important to bottom-line 
organizational performance. An interesting result was the 'perception gap' between importance and performance. The results suggest that furniture industry managers should place greater emphasis on design quality and product improvement and less on product durability, company reputation, reliability, and conformance quality, and much less to brand image and customer service. These results agree with current research in the forest products industry suggesting this sector place much greater emphasis on innovation (specifically new product development) [94, 95]. Additionally, design quality and product improvement also involved the greatest number of functions lending further support to the need for a systems-focus to quality management programs.

Forker et al. also conducted research assessing the impact of TQM on supply chain performance [38]. This study was one of the first to use DEA for such research. This paper will be discussed below in the literature review section on research using DEA for measuring quality performance.

Anderson and Sohal studied the relationship between quality management practices and performance in small businesses [96]. Their quality management measurement instrument was based primarily on the principles of the Deming Prize in Japan and the MBNQA. Performance was measured via respondents' perceptual measures of firm performance with respect to sales, exports, cash flow, employment levels, overall competitiveness and market share. A number of significant relationships were identified between TQM practices and performance, for example, 
leadership practices are particularly important for companies focused on quality rather than flexibility in delivery. A key finding from their research was in the need to capture data on needs and expectations of customers. This result appears to highlight that 'customer focus' is still seen by many firms to mean quick response to customer complaints rather than proactively seeking input and feedback from customers.

Samson and Terziovski also explored the relationships between TQM practices and operational performance [46]. The authors stated that exploring the effectiveness of TQM implementation was a key gap in the literature. The seven criteria of the MBNQA served as the TQM model elements; specifically, the authors aimed to develop constructs based on the first six criteria related to TQM practices. The measurement instrument is shown in Appendix A5. The research sought to explore the relationship of the six practices (i.e., 'inputs') to the seventh MBNQA criteria related to performance outcomes (i.e., the 'outputs'). The outcomes measured were customer satisfaction, employee morale, productivity, quality of output, and delivery performance. Factor analysis was conducted on the six practices constructs; factor scores were used as independent variables in a multiple regression analysis with the performance construct as the dependent variable. Results showed that TQM practice intensity explains a significant proportion of variance in performance. Leadership, management of people and customer focus were the strongest predictors of operational performance. The authors noted that these so called 'soft' factors were the strongest predictors of performance. It is possible that as TQM programs have evolved, the hard factors are now simply qualifying criteria; those firms able to successfully 
implement the softer factors of TQM are thus able to achieve higher levels of performance.

Hendricks and Singhal examined stock price performance of firms with effective TQM programs [41]. Winning a quality award ( 140 different awards were chosen) served as a proxy for effective TQM programs. Stock price performance was examined for five years before winning the awards and compared with performance the first five years after winning the award. There was no difference in stock price performance for the firms (and a control group) in the period before receiving the award. However after winning the award, firms significantly outperform firms in the control groups; mean buy-and-hold abnormal returns (BHARs) range from 38 percent to 46 percent. Thus, the research showed a positive correlation between quality and profitability.

Prajogo and Brown examined the relationship between TQM practices and quality performance for firms that have adopted formal quality programs vs. firms without formal quality programs [20]. In essence, TQM firms were separated from non-TQM firms based on respondents reporting whether or not they had pursued a formal TQM program. The authors used the framework developed by Samson and Terziovski (discussed above) for TQM measures and added items to the section on Benchmarking to address the company's strategy in measuring performance, availability of data and information about performance, and the use of information in decision-making processes conducted by senior management. Quality performance measures selected for the study were those developed by Ahire et al. which included 
four self-reported perceptual measures of product quality (product performance, reliability, conformance to specifications, and durability). An interesting finding in this research is that process management (composed of primarily 'hard' factors of TQM) appears to have the strongest correlation with quality performance for TQM firms, however customer focus, people management, and leadership are shown to have the strongest correlation for non-TQM firms (i.e., the 'soft' factors). These results may suggest that firms inclined to implement formal TQM programs are also more inclined to the hard factors; this further suggests the effect of organizational culture on how TQM is implemented. Further, the authors found that the lack of a formal program (as declared by survey respondents) did not necessarily equate to the absence of TQM principles. This result was somewhat unexpected in that prior studies have shown that a formal TQM campaign leads to a more organized implementation of the key elements. While there were strong links between TQM practices and quality performance, there was no significant difference between firms with formal TQM programs and those that simply adopted TQM practices (without attaching the 'label'). In short, it is the practices not the program that matter. This is further justification to study the extent to which specific practices are implemented rather than an organization's declaration of using (or not using) TQM.

In summary, numerous researchers have explored the relationships between quality and performance and most have found positive correlations. Quality inputs have been both narrowly defined (e.g., the winning of a quality award as a proxy for an effective TQM program), as well as broadly defined (e.g., the elements of the 
MBNQA). Similarly, performance has included both narrow views (e.g., percentage of parts to pass inspection) as well as broad views of performance (e.g., quality and financial metrics). Several researchers have found varying correlations between the so-called hard and soft factors of TQM and organizational performance. For example, Samson and Terziovski found that the soft factors were stronger predictors of organizational performance than hard factors. Prajogo and Brown also found that soft factors predicted performance in firms that did not identify themselves as having a formal TQM program whereas hard factors were better predictors of performance in TQM firms. In addition, these authors emphasized that the principles and practices of TQM were more important as predictors of performance than the presence or absence of the 'TQM label.' Forker et al. found that quality management practices that might also be considered to be associated with innovation (design quality and product improvement) were the most consistent predictors of organizational performance; further, these functions involve multiple functions in an organization and hence require a holistic approach, or systems-focus, for quality management. Hence, the literature exploring the relationships between quality management and organizational performance lends support to positive correlations between quality and performance and the contingency approach to the application of quality management practices with respect to hard and soft tools and scope of implementation.

However, none of the literature discussed thus far has sought to identify best quality management practices via simultaneous consideration of multiple inputs and multiple outputs. Given the suitability of DEA for such an objective, a review follows 
of studies that have used DEA in some fashion to examine impacts of quality on various measures of performance. Although few of these studies have considered quality management practices as inputs, the literature reviewed is intended primarily to present how DEA has been used in the context of research in the field of quality management.

Relationships between Quality and Performance - Studies using DEA

There are few papers reporting on the use of DEA to explore the relationships between quality and performance. Following is a summary of the papers that have explored this approach to examining quality management systems.

Mathiyalakan and Chung used DEA to evaluate the efficiency and effectiveness of quality circles [97]. They used a three-input, two-output model. Output (or what they termed effectiveness) measures included the quality circles' contribution toward organizational goals and satisfaction with quality circles; these values were perceptual data from survey respondents. Inputs (what they termed efficiency) included operating cost, quantity of presentations, and productivity. DEA analysis provided measures of relatively efficient quality circles. The authors then defined (admittedly somewhat arbitrarily) 'effective' quality circles as those with 'contribution to organizational goals' scores greater than six (based on a seven-point Likert scale). The efficient (DEA score $=1.0$ ) and effective (high score on 'contribution to organizational goals') quality circles could then be identified and used 
as benchmarks. The study is primarily exploratory in nature and intended to serve as a proposed approach to simultaneously considering efficiency and effectiveness. While the inputs and outputs have no direct application to the research proposed here, the study is presented primarily as an example of one approach to exploring efficiency and effectiveness simultaneously. However, it is not clear why the additional step of defining effectiveness is needed if the outputs are properly chosen for the DEA model.

DEA has also been used by Triantis and McNelis to evaluate quality and productivity in the pulp and paper industry [98]. With respect to quality, the association between quality costs and quality (measured as conformance and performance) was examined. Quality costs were computed as total failure costs, which are a function of prevention and appraisal costs; prevention costs include quality planning costs, employee training, supplier certification, new product reviews, etc. Appraisal costs include inspection, test and equipment calibration, evaluation of inventories, etc. The authors provide the details of the specific inputs and outputs based on data from a case firm. For example, prevention costs $=$ scheduled down time * tons/hour (of production) * profit/ton. The two measures of quality - conformance and performance - were chosen as most relevant to the case firm (as opposed to broader conceptions of quality). Data were monthly costs and quality metrics from a single manufacturing facility over a two-year period (thus the DMUs were monthly data from the same firm). The article provides only sparse details, however it appears that several separate DEA analyses were conducted such as quality performance as the 
output; inputs were labor, raw material, energy, and capital. It is unclear why a DEA model was not constructed that simply compared quality costs with quality conformance and performance as this would seem to be the most useful comparison. That is, what do you get (in terms of quality output) based on costs incurred to assure quality?

Suk used DEA to measure the impact of TQM on hospital efficiency [99]. There were two outputs (inpatients and outpatients) and seven inputs (facilities and beds as capital resources, supply costs as material resources, and senior managers/ physicians/ registered nurses, and other FTEs as labor costs). The model used was input-oriented variable-returns-to-scale. Rather than considering TQM principles and practices as inputs, the approach taken was to first determine efficient hospitals in conventional terms (cost inputs and 'product' outputs) and then to use a factorial experimental design with DEA efficiency as the dependent variable and hospital characteristics (1 measure) and TQM implementation score as the two independent variables. The measurement instruments developed by Saraph et al., Flynn et al., and Ahire et al. (see Appendix A) were adapted for use in a questionnaire for calculating a 'TQM score' for each hospital (DMU). Results were analyzed via ANOVA. As with the Mathiyalakan and Chung article described above, this research is presented here primarily as an example of DEA studies related to TQM. The specific model tested while of interest due to the use of DEA for examining TQM, is not directly relevant to the research proposed here; the study examined the impact of 'degree of TQM implementation' (conceived as a single measure) on efficiency as opposed to 
examining the impact of extent of usage of specific TQM principles and practices on quality and innovation performance. Thus the model tested is fundamentally different than what is proposed here. Suk essentially asked what relation exists between efficient firms (by traditional economic measures) and TQM. What is proposed here asks, in essence, how efficiently organizations use quality inputs to generate quality and innovation outputs.

Yoo published one of the few papers to use DEA to examine the impact of specific TQM principles and practices on quality performance [85]. Nine TQM critical success factors (i.e., TQM constructs) were considered for inputs: leadership and organization for quality, strategic quality planning, information analysis, new product development, process management, human resource management, organizational involvement, environmental and safety management, and customer satisfaction management. The output was quality performance (quality improvement level compared to domestic companies and compared to foreign companies). The Analytic Hierarchy Process (AHP) was used to assign relative importance weights to the critical success factors (both input and outputs). Based on the results, strategic quality planning, information analysis, organizational involvement, and environmental and safety management were excluded due to having relatively small weights.

The AHP analysis was based on survey responses from the firms. The survey questions were not provided in the paper and thus it is difficult to determine if the weights reflect extent of use of TQM in each DMU or the respondent's perception of 
the importance of each principle. If it is the latter, this would be a significant weakness to the paper; there could be a significant gap in what a firm states is the importance of a TQM principle and the intensity or commitment to the use of that principle. From the standpoint of assessing impact of TQM principles/ practices on performance, it would be far more valuable to measure actual use of the principles and practices within a company as inputs as opposed to perceptions of importance.

Aside from the limitations already discussed, another significant limitation for this study was the author's choice of output. 'Improvement level' says very little about quality in the sense of conformance to specifications, durability, reliability, etc. In fact, a company with very poor quality might report a large marginal gain in improvement and yet still to have a long way to go. Conversely, for a company that had already made great strides with past efforts might have a low improvement level. Further, the choice to eliminate constructs that had small AHP weights seems questionable. One must consider if the fact that a firm either places little emphasis on an input (or perceives it as of minor importance) is cause for rejecting an input that other research has validated as a key principle of TQM.

These limitations aside, a strength of the article is in using DEA to determine efficient firms and then using the practices of these firms (i.e., their AHP weight on each practice) as benchmarks for firms that were deemed 'inefficient' in their use of TQM. Unfortunately, the authors do not discuss specific best practices; the primary 
focus of the paper is on the appropriateness of the methodology for evaluating efficiency of TQM implementation rather than the results of the analysis.

Forker et al. also used DEA to evaluate the impact of TQM on performance [38]. Specifically, the research examined the impact of TQM on supply chain performance. The researchers used a unique approach to examine a supply chainone customer firm was examined along with all of its major suppliers. Inputs were the extent of implementation of TQM (using the instrument developed by Saraph et al. described above and shown in Appendix A1) based on self-reported data related to eight management practices (management leadership and quality policy, training, product/ service design, supplier quality management, process management, quality data and reporting, employee relations, and role of the quality department) for the supplier firms. Five-point Likert scale input data for each of the eight constructs is averaged to provide a single measure for each construct. The output for the model is defective parts per million; these data are the actual quality performance data for each supplier as reported by the customer firm. This use of objective data is a key strength of the study. These data are inverted and normalized to values from zero to one to avoid scaling issues.

Results of analysis via a simple linear model showed no significant relationship between the extent of TQM implementation and quality performance. The authors theorized that the small range of responses might have led to inability to distinguish among firms. The authors proposed that structural (i.e., contextual) 
differences between firms might lead to variation in efficiency in how TQM was implemented in each of the firms. This hypothesis led to the decision to use DEA to analyze the data. No details are provided on the DEA model chosen such as returnsto-scale or input/ output orientation.

Large variations in relative efficiency appeared to confirm the results of the regression analysis; there is a wide range in the efficiency values and thus perhaps gaps in how TQM practices are implemented among differing supplier firms. Stepwise regression was then used to examine the significance of TQM practices on performance (defects) as well as interaction terms between the practices and a DMU's efficiency score. Results of this phase of the analysis showed that the quality department had the largest role in assuring quality. The authors state that this finding contradicts the exhortations of such quality gurus as Ishikawa and Crosby and state that, “...these experts have claimed that quality is every employee's responsibility and that once that responsibility is adopted, the need for a quality department will disappear. Even in an industry as advanced as electronics, the quality department is of primary importance in assuring quality performance" [38]. Further, the significance of interaction terms reinforced the notion that TQM practices vary from firm to firm.

The strengths of this article are in the broad conception of TQM practices and consideration of the extent of use of these practices as inputs as well as on the use of objective data (defects) for outputs. By that same token, a key weakness lies in the narrow conception of quality performance. While the author recognizes that TQM 
practices will vary firm to firm, a key source of this variability is likely differing emphases on achieving specific quality outcomes (conformance, performance, durability, customer satisfaction, etc.). With a one-output model like this, it is not possible to identify benchmark firms and hence 'best practices' for anything beyond a single measure of quality performance (defects).

Forker et al. also published a paper describing the use of DEA as an analytical tool for benchmarking peer suppliers [84]. TQM practices, as quantified using the measurement instrument developed by Saraph et al. (see Appendix A1) served as the inputs. As with the previous study (discussed above), defective parts per million served as the output measure. This paper also does not explicitly mention details of the DEA model selected, though it appears the model was input-oriented. Results were then tallied such that DMUs referenced most often by other DMUs were determined to have the most broadly applicable TQM programs and thus could serve as good benchmarks. This is a valuable conclusion given the wide variety of approaches to implementing TQM. A firm looking for a good, 'well rounded' firm could thus benefit from this approach. The authors suggest using cluster analysis to examine the groupings to perhaps identify 'niche' approaches to implementing TQM. This suggestion has merit in that this approach could be used when studying the outputs of quality and innovation and thus testing the hypothesis that the approach to implementing TQM will vary for firms emphasizing quality performance as opposed to innovation performance and a 'best practice' cluster may well emerge for firms able to do well on both measures. One result of the research is stated as, “...many 
suppliers... achieved zero defects but with excessive expenditures on TQM inputs. 'Best peer' suppliers were able to achieve zero defects or very nearly zero defects...with minimal outlays on quality management, producing high DEA scores." Hence the method was successful in identifying those firms able to focus their TQM efforts vs. taking a 'shotgun' approach (i.e., those with 'excessive expenditures on TQM inputs).

Diaz-Balteiro et al. explored productive efficiency and innovation activity in the Spanish wood products industry using DEA [81]. While this research does not involve quality management, it is mentioned here given that the research involves DEA for examining innovation performance (i.e., as an output). The analysis was conducted by first using DEA to identify efficient firms (using relatively 'traditional' inputs and outputs) followed by logistic regression to explore correlations between efficient firms and measures of innovation.

Inputs included employees, shareholder's funds, and loans; outputs included sales and profit before taxes. Both constant returns to scale and variable returns to scale models were used to enable examination of scale efficiency. Results were tabulated to show the total number of efficient firms with and without the various measures of innovation: R\&D expenditures and partnerships, patents, and product \& process innovations. Thus, it appears the data allowed for segregation of firms with, for example, zero $R \& D$ expenditures to those with at least some nominal value expended on R\&D. 
For logistic regression, efficient firms were coded one and inefficient were coded zero. Inputs included R\&D expenditures and R\&D partnerships (a qualitative variable between zero and five depending on the number of partnerships). Outputs included patents, product innovations and process innovations. Product innovation included, “...introduction of new and significantly improved good and/or services with respect to their fundamental characteristics, technical specifications, incorporated software or other immaterial components, intended uses, or user friendliness." Process innovation was a qualitative variable equal to:

- 1 if the firm adopted one of the two types of process innovation (introduction of new and significantly improved production technologies or new and significantly improved organizational and managerial changes);

- 2 if the firm adopted both types of process innovation; and

- 0 if the firm did not adopt any process innovation.

Results (from both DEA and regression) showed a lack of links between efficiency and innovation. In fact, significant correlations were negative. For example, efficiency was inversely correlated with patents and R\&D expenditures. Again, this paper is presented here primarily because of the use of DEA for examining innovation. However, the model tested is interesting in that the premise was to first find firms efficient in an economic sense, and then determine correlations with innovation. It would be interesting to conduct the analysis again but use inputs specifically intended to lead to innovation as an output. This is not to suggest the model chosen was inappropriate as comparing 'innovation inputs' to outputs was not the authors' intent. 
In summary, few studies have been published exploring quality management using DEA. Of the articles reviewed here, only two authors (Forker et al. and Yoo) used quality management principles as the inputs; other authors have used more 'traditional' inputs such as costs. Outputs have included a variety of items such as participants' 'satisfaction with quality circles', number of hospital patients, or sales and profit data (rather than quality outputs) or narrowly defined quality outputs such as quality improvement or defect rates. The latter situation is the case even for those authors (i.e., Forker et al. and Yoo) that modeled quality management inputs in broad terms.

While there are clear advantages to using objective measures for inputs (e.g., labor and raw material costs) and outputs (e.g., product and sales revenue) when using DEA, there are challenges with respect to accurately modeling quality management with such measures. As has been discussed previously, quality management is multidimensional and involves both subjective and objective measures. Further, for the context of the research proposed here, one must consider what organizations are aiming to achieve via investing in a quality management program; 'quality-related' outputs are presumably a desired output. Thus, it is suggested here that the most appropriate input-output model for quality management considers quality management inputs and quality outputs; and if quality management inputs are broadly defined, quality outputs should be broadly defined as well. However, given widely varying approaches to implementing quality management systems and overlap with certain key principles and practices between quality management and innovation management 
(e.g., customer focus and the use of quality tools in product design), one might also pose the question, do organizations also achieve innovation performance, in addition to quality performance, as an output of a quality management system? While no studies have reported the use of DEA to address this question, there are several studies that have explored relationships between quality management and innovation performance.

\section{Relationships between Quality and Innovation}

The body of literature investigating the relationships between quality and innovation is much more recent and far less extensive than the literature exploring relationships between quality and other measures of organizational performance. As with research examining quality and organizational performance, results have been mixed regarding the 'quality - innovation connection.' Prior to discussing this research, however, literature that has explored similarities and differences between quality and innovation is examined.

Kanji put it most succinctly in proposing that TQM and innovation were aligned - both are focused on delighting customers and further, suggested that successful innovation depends on TQM [34]. Though the premises are not tested, the author proposes that the key link may be to integrate long and short-term strategies.

In contrast, Samaha proposes that TQM will diminish avenues for innovation given that it reinforces incremental vs. breakthrough innovation [31]. The author 
states "Quality is doing things better; innovation is doing things differently."

Benchmarking is seen as 'perilous' with respect to innovation in that benchmarking is a means to 'catch up' to the competition. TQM prevents thinking outside the box and provides negative reinforcement for risk-taking. These premises are not tested in the paper. It is suggested that innovation be embraced as part of the quality effort.

A contingency perspective for TQM is presented by Sitkin et al. [24]. It is proposed that differences in application of key TQM principles are based on degree of contextual uncertainty - low levels of uncertainty are amenable to a focus on control (i.e., TQC, Total Quality Control) in TQM whereas higher levels of uncertainty lead to the need to emphasize TQL - Total Quality Learning. While the focus of the study is not strictly on the relationship to innovation, the connection between innovation and learning is alluded to by stating that firms must balance stability and reliability (i.e., control) with exploration and innovation (i.e., learning). The principles of TQC and TQL are summarized in Table 3. 
Table 3. Distinctive Principles Associated with TQC and TQL Compared to Common TQM Precepts [24]

\begin{tabular}{|c|c|c|}
\hline $\begin{array}{l}\text { Shared TQM } \\
\text { Precepts }\end{array}$ & $\begin{array}{l}\text { Control-Oriented Principles } \\
\text { (TQC) }\end{array}$ & Learning-Oriented Principles (TQL) \\
\hline $\begin{array}{l}\text { Customer } \\
\text { Satisfaction }\end{array}$ & $\begin{array}{l}\text { Monitor and assess known } \\
\text { customer needs } \\
\text { Benchmark to better understand } \\
\text { existing customer needs } \\
\text { Respond to customer needs }\end{array}$ & $\begin{array}{l}\text { Scan for new customers, needs, or } \\
\text { issues } \\
\text { Test customer need definitions }\end{array}$ \\
\hline $\begin{array}{l}\text { Continuous } \\
\text { improvement }\end{array}$ & $\begin{array}{l}\text { Exploit existing skills and } \\
\text { resources } \\
\text { Increase control and reliability }\end{array}$ & $\begin{array}{l}\text { Explore new skills and resources } \\
\text { Increase learning and resilience }\end{array}$ \\
\hline $\begin{array}{l}\text { Treating the } \\
\text { Organization as a } \\
\text { Total System }\end{array}$ & $\begin{array}{l}\text { First-order learning (cybernetic } \\
\text { feedback) } \\
\text { Participation enhancement focus }\end{array}$ & $\begin{array}{l}\text { Second-order learning } \\
\text { Diversity enhancement focus }\end{array}$ \\
\hline
\end{tabular}

Prajogo and Sohal discuss arguments in support of a positive, as well as a negative, relationship between TQM and innovation [30]. These are premises based on the key principles underlying TQM and innovation rather than empirically verified results from research studies. They summarize this information as shown in Table 4. The many similarities between the negative arguments and Sitkin et al.'s suggestions for learning-oriented principles can lead to an understanding of possible factors limiting the impact of TQM on innovation performance. Some of these elements have been addressed previously with respect to the dualities within TQM, e.g., being reactive rather than proactive with respect to customer needs and narrowly defining the customers as existing customers vs. previously unserved needs and markets. 
Table 4. Summary of Conflicting Arguments on the Relationship Between TQM and Innovation [30]

\begin{tabular}{|c|c|c|}
\hline TQM Elements & Positive Arguments & Negative Arguments \\
\hline Customer focus & $\begin{array}{l}\text { Customer focus: } \\
\text { - will encourage } \\
\text { organizations to be } \\
\text { innovative because they } \\
\text { have to seek a better way } \\
\text { to meet and exceed } \\
\text { customers' requirements } \\
\text { - will provide a clear focus } \\
\text { for innovation by linking } \\
\text { innovation with } \\
\text { customers' needs }\end{array}$ & $\begin{array}{l}\text { Customer focus: } \\
\text { - } \quad \text { could lead organizations to be reactive in } \\
\text { responding to customers' needs } \\
\text { may prevent organizations from exploring } \\
\text { unserved needs and markets } \\
\text { may prevent organizations from developing } \\
\text { radical new products (first-mover) because } \\
\text { of its inherent risk-avoidance philosophy } \\
\text { - could not help organizations to cope with } \\
\text { turbulence and discontinuity of the market }\end{array}$ \\
\hline $\begin{array}{l}\text { Continuous } \\
\text { improvement }\end{array}$ & $\begin{array}{l}\text { Continuous improvement: } \\
\text { - will encourage change, } \\
\text { innovation, and creative } \\
\text { thinking in how work is } \\
\text { organized and conducted }\end{array}$ & $\begin{array}{l}\text { Continuous improvement: } \\
\text { - } \quad \text { emphasis of efficiency would minimize, if } \\
\text { not eliminate, the availability of slack } \\
\text { resources that are required for innovation } \\
\text { stress on incremental improvement could } \\
\text { lead teams to work on unambitious goals } \\
\text { and derive solutions which are not novel } \\
\text { is only workable when the underlying } \\
\text { system of production is stable and } \\
\text { repetitive, and not in a particular } \\
\text { environment where there is a high degree of } \\
\text { uncertainty } \\
\text { the establishment of a regulatory standard } \\
\text { could inhibit innovation because it reduces } \\
\text { the ambiguity of a task that is necessitated } \\
\text { to enforce innovation } \\
\text { could also result in routinization and rigidity } \\
\text { of activities that will cause an organization } \\
\text { to lose its flexibility } \\
\text { only supports single-loop learning and not } \\
\text { double-loop learning }\end{array}$ \\
\hline $\begin{array}{l}\text { Teamwork, } \\
\text { empowerment and } \\
\text { involvement }\end{array}$ & $\begin{array}{l}\text { Empowerment should } \\
\text { make people feel they } \\
\text { have a certain degree of } \\
\text { autonomy, are less } \\
\text { constrained by technical } \\
\text { or rule-bound aspects, } \\
\text { and self-efficacious in } \\
\text { doing their work, which } \\
\text { will make them } \\
\text { innovative } \\
\text { Cross-functional } \\
\text { teamwork is one of the } \\
\text { most effective channels } \\
\text { of communication, and } \\
\text { communication is } \\
\text { recognized as the primary } \\
\text { determinant in } \\
\text { organizational innovation }\end{array}$ & $\begin{array}{l}\text { - Whilst conceptually empowerment and } \\
\text { involvement are very much congruent with } \\
\text { innovation, in practice, workers are usually } \\
\text { "empowered and involved" to deal only } \\
\text { with execution and small scale of } \\
\text { improvement } \\
\text { The cultural tendency toward group } \\
\text { working which has contributed towards } \\
\text { stressing total quality control to a certain } \\
\text { degree will inhibit independent } \\
\text { entrepreneurship and individual creativity, } \\
\text { resulting in a detrimental effect upon radical } \\
\text { innovations and inventions }\end{array}$ \\
\hline
\end{tabular}


There are thus, good reasons to believe there may be positive, as well as negative correlations between quality management practices and innovation performance. Given the importance of both quality and innovation to organizational performance, the question of relationships between these two fields is highly relevant for managers. Further, given the investments most organizations have made in quality management, it would be valuable for managers to know which, if any, specific aspects of quality management lead to enhanced innovation performance. While the preceding studies have proposed relationships, the studies did not test these relations. Hence, attention is now directed to the literature that has empirically examined these relations.

Lin and Lu explored a causal relationship between quality and innovation in examining product innovation in the automotive industry [80]. Number of patents served as the metric for innovation and problems-per-100 vehicles served as the measure of quality. Regression analysis was used to model product quality as the independent variable and innovation as the dependent variable. Results showed that automakers actively engage in product innovation if they produce high-quality products. The authors thus proposed that product quality determines product innovation. The key limitation to this research was the unidimensional view of both quality and innovation.

Bossink conducted a case study to examine the use of specific quality tools for an innovative homebuilding project in the Netherlands [100]. Results suggest a 
supportive function of quality in the management of innovation. Further, it is suggested that quality tools are used implicitly and sometimes explicitly to manage innovation, for example, benchmarking, customer satisfaction measurement, and teams are used in both quality as well as innovation management. A key finding was that measurement of customer satisfaction contributed to the initiation of innovation; this might be seen as more of a proactive approach to customer focus. One limitation of the study was in accurately identifying the use of specific quality tools. That is, 29 'quality tools' were examined for how they were used in the innovation project. Some of these tools were in fact 'concepts', as the authors acknowledge in the caption of the table listing the items. Thus, while one may be able to state with some certainty whether or not a specific tool was used in a situation, it is less clear how to identify the use, or lack of, of a broad concept or philosophy. Further, the authors merely state whether or not a tool was used and in what stage of a project (creating innovation context, supervising innovation processes, etc.) without providing detail as to how the tools were used.

McAdam et al. examined organizational progression from quality to innovation [23]. The European Foundation for Quality Management's (EFQM) European Quality Model served as the model for quality management and the Centrim innovation model (Centre for Research in Innovation Management, University of Brighton) served as the model for innovation. The authors developed a list of assumptions underlying TQM (e.g., continuous improvement, cause and effect/ enablers and results, limited empowerment, etc.) and innovation (continuous renewal, 
new ideas not extrapolation of past, proactive activity, etc.). Using the main categories of the EFQM and Centrim models, a matrix was developed to identify if each category was related to TQM, innovation, or both. Fifteen firms were then evaluated based on the EFQM (quality) and Centrim (innovation) criteria and given a score for innovation and continuous improvement. The authors report that there were significant similarities between firms that got high scores on both quality and innovation. The authors state that Total Quality “...lays the foundation for a cultural environment that encourages innovation." A key limitation of the study was in narrowly defining Total Quality as practices focused on continuous improvement.

Singh and Smith explored “... whether TQM can be used as a vehicle for inculcating innovation" [33]. The authors adapted their TQM measurement instrument (see Appendix A4, [70]) to serve as the key constructs for measuring TQM implementation. The four measures of innovation discussed previously included: innovative processes/ products/ services have been commercialized; R\&D leads to development of world-class techniques/ technologies; the rate of innovation of new operational processes; and the rate of introduction of new products and services.

Firms were surveyed and asked to evaluate their performance relative to the TQM and innovation criteria. Structural equations modeling (SEM) was used to analyze the structural model that at its essence simply hypothesized that TQM (conceived as a series of seven constructs) leads to innovation. The authors concluded there was insufficient evidence to link TQM and innovation and suggested "It could 
well be that the TQM constructs and innovation are related in a more complex way. Indeed, business excellence frameworks... postulate that the constructs are interrelated in complex ways." This lends further support to the use of DEA in that these interrelations would not impact a DEA model. Further, an unexpected result of the test for construct validity in the research was that the construct Product/ Process Management led to poor fit of the model; use of SPC in particular was eliminated from the model. Given that product/ process management are key factors in TQM, and SPC is a key tool used, the reason for this poor fit should be explored further. Lastly, the model the authors used appears to be inconsistent in one instance with respect to 'cause and effect.' Specifically, the independent variables proposed are related to quality management; however one of the dependent variables is attributed to $R \& D$ as the cause. Perhaps the intent was to understand if TQM practices applied in R\&D enhanced innovation performance. At the least, this inconsistency may have led to inconclusive results.

In addition to proposing a list of arguments for both positive and negative relationships between TQM and innovation (Table 4), Prajogo and Sohal proposed that the key differences in how TQM principles were applied in a firm were related to differences in external context (business environment), organizational strategy, and organizational culture [30]. For example, following arguments from Sitkin et al., a stable external environment might lead to a control and hence 'quality focus' for a firm, whereas a more volatile business environment might lead a firm to emphasize innovation [24]. Organizational culture (e.g., mechanistic vs. organic) could be 
manifested in the choice of hard vs. soft tools. And a differentiation strategy might lead to a focus on product innovation, whereas a cost leader strategy might lead to emphasizing process innovation. The authors propose the research framework shown in Figure 6 to study these relationships [30]. Additionally, for purposes of studying performance, the authors state that TQM constructs and accompanying measurement instruments developed by Saraph et al. [67], Flynn et al. [60], Ahire et al. [50], and Samson and Terziovski [46], all appear to primarily address conformance quality again, the view of quality from the manufacturer-based viewpoint that quality is equivalent to meeting specifications. Hence, they propose re-examining these constructs for their applicability to broader conceptions of quality.

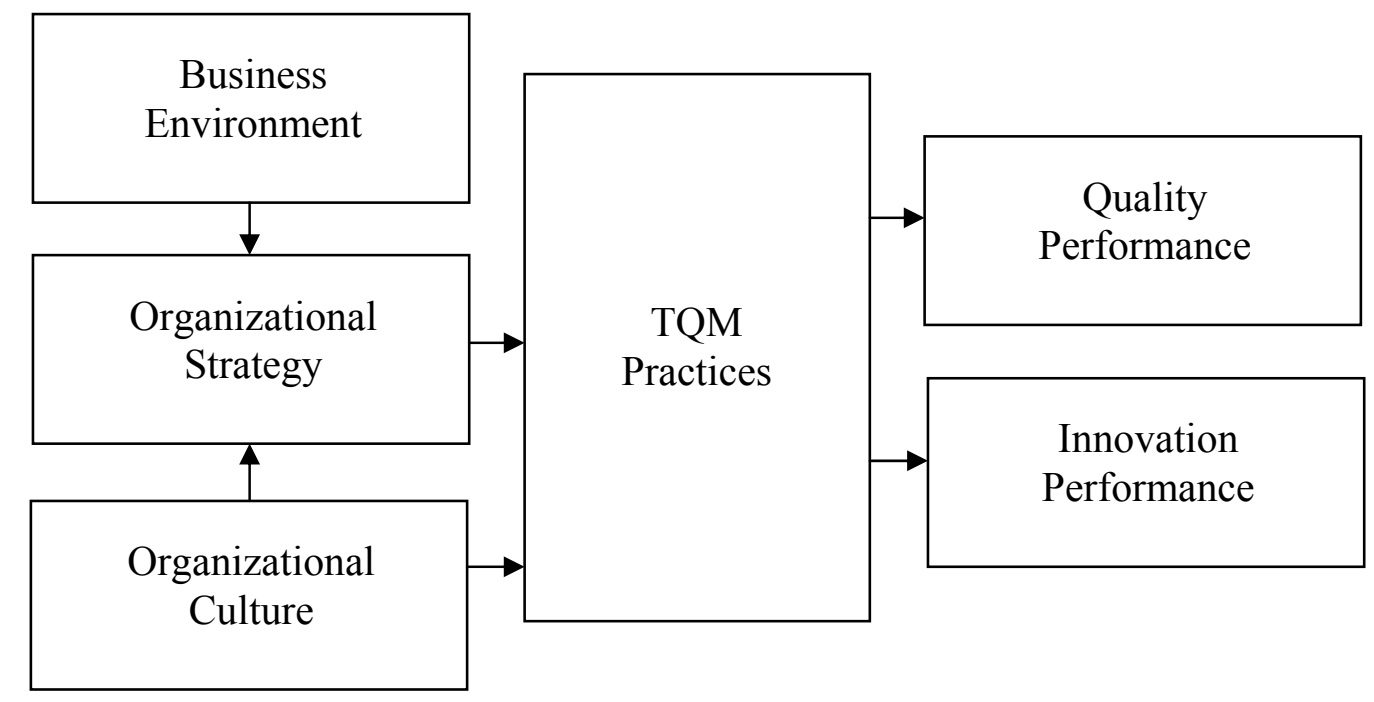

Figure 6. TQM and Innovation: Research Framework [30]

Prajogo and Sohal have published several papers exploring the relationships proposed in Figure $6[25,35,45,82]$. In their first published report on the topic, they surveyed firms to evaluate their quality management practices using the constructs 
developed by Samson and Terziovski shown in Appendix A5 [46]; however the Information and Analysis construct was adapted to better reflect the MBNQA ('Baldrige Award') criteria for this topic. Specifically, questions were added on company strategy in measuring performance, availability of data and information about performance, and use of information in decision-making processes conducted by senior management. Quality performance was measured using constructs developed by Ahire et al. [50] and thus included multiple dimensions of quality (reliability, performance, durability, and conformance to specifications). Innovation performance was measured using the constructs shown in Table 2 above.

Structural equations modeling (SEM) was used to examine the simultaneous relationship between TQM and product quality performance, product innovation performance, and process innovation performance. Results showed that TQM significantly and positively relates to quality performance as well as innovation performance. As would be expected, the relation between TQM and quality was stronger than the relation between TQM and innovation. Further, there are significant correlations between product quality, product innovation, and process innovation; though the relation between product quality and product innovation was weaker than the other two correlations. The authors suggested the possibility of 'crossfertilization' between quality and process innovation performance; process innovation may mediate the relationship between product quality and product innovation. This suggestion seems likely in that, forest industry researchers have shown that process automation technology such as scanning systems (i.e., one form of process innovation) 
is often pursued with the purpose of reducing variability, i.e., improving the 'product conformance' dimension of quality [52, 101, 102]. A final point is offered that, “...care needs to be taken before claiming that TQM in its own right is sufficient for achieving high innovation performance..." and further that “...quality management is a prerequisite for innovation management..." This caution proved valid in that results of this study were contradicted in a later paper by these authors [35].

Prajogo and Sohal also conducted a case study in an Australian firm to examine the transition from TQM to 'Total Innovation Management' [82]. One key finding in this study is that this transition resulted in shifting many of the tools of quality management from downstream operations (i.e., manufacturing processes) to upstream operations (i.e., design and development). This again addresses one of the key principles in TQM - the emphasis on total, that is, implementing the practices company-wide. Though this was the intent of TQM from the very beginning, the literature seems to indicate that many companies have been slow to realize (or at least implement) this principle. The authors further state that the transition in the case study firm resulted in customer relationship management shifting from reactive (responding to customer complaints) to being proactive as well as to shifting its focus from direct customers to end users. A final conclusion of interest was the potential 'chain reaction' between process innovation and product innovation; the firm implemented automation (process innovation) to improve product quality and was then 'forced' to innovate new products that were suited to the technology. The primary limitation of 
this study was in the use of a single firm for the case study and thus no ability to use cross-case comparisons for validation.

Prajogo and Sohal extended their research exploring relationships between quality management and innovation performance by studying the impact of integration of TQM and Total Innovation Management (TIM) on performance [35]. TIM is defined to include technology management and R\&D management. Citing Benner and Tushman [103], the authors highlight the importance of organizations developing capacities to simultaneously balance exploitation and exploration. Exploitation is defined as an organization's capability to maximize existing resources for efficiency and productivity and hence is at its essence the focus of quality management. Exploration involves maximization of capabilities to develop new skills and resources, and hence is the focus of innovation management.

As with previous research by these authors, firms were surveyed to determine their performance with respect to quality and innovation; the constructs used for these measures were the same as in prior papers (described above), however new constructs (shown in Table 5) were developed to evaluate technology and R\&D management. The new constructs were tested and shown to be reliable.

Structural equations modeling was used to examine the relationships. Findings for relations to quality performance agreed with those from previous research by these authors - TQM showed strong predictive power over quality performance. However, the results contrasted with their prior research on relationships with innovation - TQM 
showed no significant relationship with innovation performance. TIM was also shown to be related to quality performance, although the correlation was weaker than for TQM and quality performance; TIM showed a strong relationship to innovation performance. Further, there was a strong and positive correlation between TQM and TIM. The authors state that this latter finding confirms their hypothesis that TQM and TIM are compatible and further, results suggest that TQM and TIM operate synergistically.

Table 5. Constructs for Measuring Technology and R\&D Management [35]

\begin{tabular}{|c|c|}
\hline Technology Management & R\&D Management \\
\hline $\begin{array}{l}\text { - Our company always attempts to stay on } \\
\text { the leading edge of new technology in } \\
\text { our industry } \\
\text { - We make an effort to anticipate the full } \\
\text { potential of new practices and } \\
\text { technologies } \\
\text { We pursue long-range programmes in } \\
\text { order to acquire technological } \\
\text { capabilities in advance of our needs } \\
\text { We are constantly thinking of the next } \\
\text { generation of technology }\end{array}$ & $\begin{array}{l}\text { - We have excellent communication } \\
\text { processes between R\&D and other } \\
\text { departments. } \\
\text { - } \quad \text { Our R\&D pursues truly innovative and } \\
\text { leading-edge research. } \\
\text { - } \quad \begin{array}{l}\text { Our R\&D strategy is mainly characterized } \\
\text { by high risk projects with chance of high }\end{array} \\
\text { return. } \\
\text { R\&D plays a major part in our business } \\
\text { strategy. }\end{array}$ \\
\hline
\end{tabular}

Significant relations were also shown to exist between product quality and process innovation and between product innovation and process innovation. The authors repeat their findings from earlier research that this suggests 'crossfertilization' between these performance variables. For example, firms might pursue process innovation to reduce variability, and thus improve product conformance quality. And a new product innovation might require process innovation to be successfully manufactured. 
Prajogo and Sohal conclude that technology/ R\&D management should be used in harmony with TQM. The authors proposed two examples where principles of TQM might be integrated with TIM:

- Customer focus - organizations should strive to balance efforts to meet current customer needs with R\&D focused on meeting unserved needs, i.e., more of a proactive approach to customer focus. Similarly, organizations should serve customer needs through product innovation that is technology push rather than market pull.

- Process management - organizations should strive to balance control and continuous improvement with technology management that is more focused on radical innovation. No further details are given regarding this point.

The practical implications of this research are critical to identifying best practices for quality management that lead to both quality and innovation performance. The authors propose two approaches to integrating TQM and technology/ R\&D management. It is important to note that the proposed changes are seen from the perspective of the fundamental TQM principles of customer focus and process management. This, combined with the authors' statement that “...quality management is the 'pre-requisite' of innovation management", suggests that one may conceive of these changes as being primarily an adaptation of the existing quality management system. That is, assuming a firm has an existing technology management and R\&D program, these are presumed sufficient to generate innovation 
performance; however, shifting the focus of the TQM program will serve to better align and integrate quality management with innovation management.

Hoang et al. explored the relationships between TQM and innovation, and went further to address the impacts of specific TQM practices [104]. Based on a review of the literature, eleven TQM constructs were developed:

1. Top management commitment

2. Employee involvement

3. Employee empowerment

4. Education and training

5. Teamwork

6. Customer focus

7. Process management

8. Information and analysis system

9. Strategic planning

10. Open organization

11. Service culture

These constructs were selected as they, “...represent the hard and soft aspects of TQM...", cover quality award criteria, and are considered critical practices in TQM for both service and manufacturing organizations. Thus for the former, there is alignment with the idea of one of the primary dualities presented here for TQM - hard vs. soft aspects. The authors do not explicitly define how the constructs were operationalized, however the authors mention the use of questionnaires and references are made to prior authors such as Samson and Terziovski and Ahire et al. Thus it must be assumed that self-reported perceptual data, as has been done by these and 
other researchers, were used to quantify intensity of use of specific TQM practices in respondent firms.

Innovation was measured in terms of:

1. Number of new products/ services the firm had developed and commercialized over the last three years; share of current annual turnover that sales of these innovation products/ services had generated and

2. Level of newness using a modified version of the scale developed by Johannessen et al. ([76], see Appendix B1). The modifications included combining new product and new service into one dimension and rewording other dimensions. The resulting constructs for innovation included:

- Entirely new product or new service

- Use of new materials or intermediate products

- New functional solution for an existing product or additional service based on an existing service

- New method of production

- Entering a new market

- New source of supply

- New ways of organizing (re-arranging the company's human resource)

Respondents rated their firm on a scale from one to five to represent the degree to which their firm had made changes within the last three years to any of these items within their firm. By considering only changes within the firm, using the arguments proposed by Johannessen et al., the authors implicitly considered innovations that were more incremental in nature (i.e., by defining 'newness' as new to the firm) rather than more radical innovations new to the industry.

A pilot study was conducted to ensure the internal consistency and reliability of the constructs. Firms to be surveyed were selected based on their registration to 
ISO 9001. This is a potential limitation of the study in that such firms may not be representative of the broader population; thus, the results may not be generalizable. The results of the pilot study suggested retaining all constructs with the exception of Strategic Planning. Following the full survey, confirmatory factor analysis (CFA) was used to examine construct validity. As a result, five items (total) in the Teamwork, Process Management, and Information and Analysis measures were removed due to poor loading on their respective latent variables (constructs).

CFA was also used to study the relationship between TQM and innovation. Specifically, the relationship examined was the impact of TQM (as measured by 11 constructs) on level of newness (as measured by seven constructs), number of new products, and share of current year sales from new products. Results showed that TQM has a strong and positive relationship with level of newness and number of new products and services developed. However there was no relationship between TQM and the share of current year sales contributed by the new products.

Finally, in order to explore the relationship between specific TQM practices and innovation, Hoang et al. used CFA to examine all possible relationships between the 11 TQM constructs and level of newness as well as number of new products. Results showed significant problems with multicollinearity within the TQM constructs. To address this problem, the authors developed composite measures: Leadership and People Management (including the constructs for top management commitment, employee involvement, and employee empowerment) and Process and 
Strategic Management (including the constructs for process management, information and analysis system, and strategic planning). It is not clear if the latter construct was an error in that the authors had stated earlier that strategic planning had been removed following the pilot study to check for internal consistency and reliability. The authors report that Leadership \& People Management relates to the human aspects of TQM while Process \& Strategic Management reflects the mechanistic aspects of TQM.

Using the composite measures, the analysis revealed that Leadership \& People Management was significantly related to level of newness and Open Organization (item number ten in the list of constructs, i.e., apparently not included as a composite measure) was significantly related to number of new products and services. Only Education \& Training and Process \& Strategic Management had significant relationships with both measures of innovation. The authors concluded that not all TQM factors have an impact on innovation performance.

In summary, several authors have proposed positive as well as negative relations between quality and innovation based on the shared precepts of both fields $[24,30,31,34]$. For example, customer focus, continuous improvement, or teamwork can either negatively or positively impact innovation performance depending on how they are viewed by, and implemented in, an organization.

Several authors have conducted empirical research exploring relationships between quality and innovation $[23,25,33,35,45,80,82,100]$. Results have been 
mixed. There are positive correlations such as a supporting role for quality in the management of innovation, suggestions that TQM lays the foundation for innovation, and straightforward assessments that TQM significantly and positively impact quality and innovation performance. However, Singh and Smith reported insufficient evidence of a relationship between quality and innovation - perhaps due to a more complex model [33].

Prajogo and Sohal may have addressed this 'more complex model' by including constructs for TIM (total innovation management - defined as technology management and R\&D) as inputs to their model [35]. In prior research, Prajogo et al. reported that TQM significantly and positively impacted quality and innovation performance $[25,45,82,105]$. However, when constructs for R\&D and technology management were included in the model there were no significant correlations between TQM and innovation performance, although there were significant correlations between TQM and TIM. The authors describe the synergy between quality management and innovation management and hence the importance of integrating principles and practices within the two fields. As discussed above, the authors' proposed approaches to integration of TQM and TIM may be viewed from the standpoint of how to adapt TQM principles and practices for better alignment with existing approaches to innovation management.

Table 6 below summarizes the literature exploring relationships between quality and innovation. Those authors that proposed relationships (Kanji [34], Samaha 
[31], Sitkin [24], Prajogo and Sohal [30]) but did not empirically test the relationships, are not shown in the table. 
Table 6. Summary of Literature Exploring Relationships between Quality and Innovation

\begin{tabular}{|c|c|c|c|c|}
\hline Authors & Context & $\begin{array}{l}\text { Research } \\
\text { method }\end{array}$ & Findings & Limitations \\
\hline $\begin{array}{l}\text { Lin and } \mathrm{Lu} \\
{[80]}\end{array}$ & $\begin{array}{l}\text { Automotive } \\
\text { (global) }\end{array}$ & $\begin{array}{l}\text { Regression } \\
\text { (innovation } \\
\text { as dependent } \\
\text { variable; } \\
\text { quality as } \\
\text { independent } \\
\text { variable) }\end{array}$ & $\begin{array}{l}\text { Automakers engage in product } \\
\text { innovation if they produce } \\
\text { high-quality products (i.e., } \\
\text { product quality determines } \\
\text { product innovation) }\end{array}$ & $\begin{array}{l}\text { Uni-dimensional } \\
\text { measures of quality } \\
\text { and innovation }\end{array}$ \\
\hline $\begin{array}{l}\text { Bossink } \\
{[100]}\end{array}$ & $\begin{array}{l}\text { Innovative } \\
\text { homebuilding } \\
\text { project } \\
\text { (Netherlands) }\end{array}$ & $\begin{array}{l}\text { Case study } \\
\text { (examination } \\
\text { of use of } \\
\text { quality tools } \\
\text { in project) }\end{array}$ & $\begin{array}{l}\text { Supportive function of quality } \\
\text { in management of innovation; } \\
\text { customer satisfaction } \\
\text { measurement contributes to } \\
\text { innovation }\end{array}$ & $\begin{array}{l}\text { Unclear how tool } \\
\text { usage was } \\
\text { determined (some } \\
\text { 'tools' were } \\
\text { broader concepts); } \\
\text { no mention of how } \\
\text { tools were used }\end{array}$ \\
\hline $\begin{array}{l}\text { McAdam et } \\
\text { al. [23] }\end{array}$ & $\begin{array}{l}\text { Small } \\
\text { organizations } \\
\text { (Ireland) }\end{array}$ & $\begin{array}{l}\text { Score firms } \\
\text { using } \\
\text { European } \\
\text { Foundation } \\
\text { for Quality } \\
\text { Management } \\
\text { and Centrim } \\
\text { Innovation } \\
\text { models } \\
\end{array}$ & $\begin{array}{l}\text { Total Quality lays foundation } \\
\text { for innovative culture } \\
\text { (similarities between firms } \\
\text { getting high scores on both } \\
\text { quality and innovation) }\end{array}$ & $\begin{array}{l}\text { Total Quality } \\
\text { narrowly defined as } \\
\text { continuous } \\
\text { improvement }\end{array}$ \\
\hline $\begin{array}{l}\text { Singh and } \\
\text { Smith [33] }\end{array}$ & $\begin{array}{l}\text { Manufacturing } \\
\text { (Australia) }\end{array}$ & $\begin{array}{l}\text { Survey, } \\
\text { Structural } \\
\text { Equations } \\
\text { Modeling }\end{array}$ & $\begin{array}{l}\text { Insufficient evidence to link } \\
\text { TQM and innovation; may be } \\
\text { more complex relations }\end{array}$ & $\begin{array}{l}\text { Product/ Process } \\
\text { Management } \\
\text { construct led to } \\
\text { poor fit - SPC } \\
\text { eliminated from } \\
\text { model (though this } \\
\text { is a key tool of } \\
\text { quality } \\
\text { management); } \\
\text { Independent } \\
\text { variables are for } \\
\text { quality mgmt. but } \\
\text { one dependent } \\
\text { variable related to } \\
\text { R\&D }\end{array}$ \\
\hline $\begin{array}{l}\text { Prajogo and } \\
\text { Sohal [25] }\end{array}$ & $\begin{array}{l}\text { Organizations } \\
- \text { mfg and } \\
\text { service } \\
\text { (Australia) }\end{array}$ & $\begin{array}{l}\text { Survey, } \\
\text { Structural } \\
\text { Equations } \\
\text { Modeling }\end{array}$ & $\begin{array}{l}\text { TQM significantly and } \\
\text { positively related to quality and } \\
\text { innovation performance; } \\
\text { significant correlations } \\
\text { between product quality, } \\
\text { product innovation, and } \\
\text { process innovation; possible } \\
\text { 'cross-fertilization' between } \\
\text { quality and process innovation } \\
\text { performance }\end{array}$ & $\begin{array}{l}\text { Focus on } \\
\text { relationships vs. } \\
\text { specific practices }\end{array}$ \\
\hline
\end{tabular}




\begin{tabular}{|c|c|c|c|c|}
\hline Authors & Context & $\begin{array}{l}\text { Research } \\
\text { method }\end{array}$ & Findings & Limitations \\
\hline $\begin{array}{l}\text { Prajogo and } \\
\text { Sohal [82] }\end{array}$ & $\begin{array}{l}\text { Large } \\
\text { manufacturing } \\
\text { firm } \\
\text { (Australia) }\end{array}$ & $\begin{array}{l}\text { Case study; } \\
\text { transition } \\
\text { from TQM to } \\
\text { TIM (total } \\
\text { innovation } \\
\text { management) }\end{array}$ & $\begin{array}{l}\text { TQM } \rightarrow \text { TIM transition resulted } \\
\text { in shifting quality management } \\
\text { tools from mfg upstream } \\
\text { operations (i.e., more holistic } \\
\text { implementation); customer } \\
\text { relationship management shift } \\
\text { from reactive to proactive; } \\
\text { customer view shift from direct } \\
\text { customers to end users }\end{array}$ & $\begin{array}{l}\text { Single firm (i.e., } n \\
=1 \text { ) }\end{array}$ \\
\hline $\begin{array}{l}\text { Prajogo and } \\
\text { Sohal [35] }\end{array}$ & $\begin{array}{l}\text { Organizations } \\
\text { - mfg and } \\
\text { service } \\
\text { (Australia) }\end{array}$ & $\begin{array}{l}\text { Survey, } \\
\text { Structural } \\
\text { Equations } \\
\text { Modeling }\end{array}$ & $\begin{array}{l}\text { TQM related to quality } \\
\text { performance but not innovation } \\
\text { performance; innovation } \\
\text { management related to quality } \\
\text { (weakly) and innovation } \\
\text { performance; strong correlation } \\
\text { between TQM and innovation } \\
\text { management }\end{array}$ & $\begin{array}{l}\text { Focus on general } \\
\text { relationships vs. } \\
\text { specific practices }\end{array}$ \\
\hline $\begin{array}{l}\text { Hoang et al. } \\
{[104]}\end{array}$ & $\begin{array}{l}\text { Organizations } \\
- \text { mfg and } \\
\text { service } \\
\text { (Vietnam) }\end{array}$ & $\begin{array}{l}\text { Survey, } \\
\text { Structural } \\
\text { Equations } \\
\text { Modeling }\end{array}$ & $\begin{array}{l}\text { TQM has strong \& positive } \\
\text { relationship with level of } \\
\text { newness and number of new } \\
\text { products and services } \\
\text { developed; no relation between } \\
\text { TQM and share of current year } \\
\text { sales contributed by new } \\
\text { products; Leadership \& People } \\
\text { Management construct related } \\
\text { to level of newness; Open } \\
\text { Organization related to number } \\
\text { of new products and services; } \\
\text { Education \& Training as well } \\
\text { as Strategic Management } \\
\text { related to both measures of } \\
\text { innovation }\end{array}$ & $\begin{array}{l}\text { Problems with } \\
\text { multicollinearity } \\
\text { when attempting to } \\
\text { explore relations } \\
\text { between specific } \\
\text { practices (led to use } \\
\text { of composite } \\
\text { measures); target } \\
\text { firms ISO } 9000 \\
\text { registered (i.e., } \\
\text { possibly not } \\
\text { representative of all } \\
\text { organizations) }\end{array}$ \\
\hline
\end{tabular}

A key limitation to the research conducted to date related to exploring the impacts of TQM on performance is lack of detail in how specific principles and practices (i.e., the constructs of leadership, strategic planning, customer focus, etc.) are related to performance. The study by Hoang et al. described above is one exception. However, as described by Hoang et al., significant multicollinearity among the constructs led to the need to use highly aggregated (i.e., composite) measures in order to analyze the data using multivariate methods such as confirmatory factor analysis. 
Hence the ability to examine impact of specific TQM principles on performance is limited. Given the multitude of demands placed on managers, it would be most useful to know which areas of emphasis are most likely to lead to improved quality and innovation performance as opposed to simply knowing whether or not TQM (as an integrated set of practices), or in a best-case scenario, highly-aggregated sets of TQM principles, positively impact performance.

\section{Gaps in the Literature}

The preceding review of the literature leads to identification of several significant gaps, including:

1. The research exploring the impact of quality management on both quality and innovation performance is sparse;

2. Much of the published studies do not adequately address the multidimensional nature of both quality and innovation; and

3. Research has focused on exploring relationships between TQM (often as a single construct) and organizational performance rather than the individual quality management principles and practices; further the focus has been on relationships rather than best practices.

The first point regarding the paucity of research discussing the "qualityinnovation connection' is not surprising if one accepts the premise that organizations have only recently begun to enter a transitional phase from a focus on quality to a focus on both quality and innovation. If, as some experts have suggested, quality is a necessary but insufficient condition for organizational competitiveness, and innovation is the 'next competitive frontier', then successful organizations must focus on 
managing for both outputs. Based on the literature reviewed here, the importance of the topic was presented as early as 1996; however the majority of the literature exploring the relationships between quality and innovation has been published in the last ten years. This suggests the topic is timely and that there are opportunities for further exploration.

Regarding the second point, many researchers have discussed the multidimensional nature of quality and innovation. However, a significant portion of the literature examining quality performance has conceived of quality primarily from the perspective of conformance to specifications, e.g., product defect rates. Innovation performance has often been measured via metrics such as number of patents or new products released. Measuring quality and innovation too narrowly fails to account for the contingency approach by which firms with differing strategies, cultures, and contexts pursue the various dimensions of quality and innovation.

Further, in discussing the dualities within TQM above (Figure 3), the research suggests that many firms have focused on a narrow conception of TQM, e.g., the use of 'hard' tools for process improvement in manufacturing, rather than true Total Quality Management. Measurement instruments developed to assess the extent of implementation of TQM in organizations have implicitly addressed the use of both hard and soft tools; however it is less clear if elements of internal vs. external focus or scope of implementation are effectively addressed. In particular, customer focus has been vaguely defined such that it is not clear if it is being addressed reactively or 
proactively. This may be a critical distinction from the standpoint of innovation performance.

Also with respect to exploration of relationships between quality management and performance, there is a significant gap in connecting specific elements of quality management to performance. TQM has often been measured as a broad construct composed of factors related to leadership, people management, process management, etc. Thus, researchers have been able to determine the presence and significance of relationships between TQM (as a broad construct) and innovation, however little attention has been given to relationships between specific TQM practices and performance. Part of the challenge in doing so has been the limitations of the research methods to accommodate multicollinearity among factors. A common approach to address multicollinearity is to combine correlated factors into composite factors; however highly-aggregated factors make it difficult to determine where to focus efforts for improvement.

A final gap in the literature is identification of best practices. Given the importance of benchmarking as a practice within TQM, it seems logical to benchmark TQM practices themselves. In this case, benchmarking would be used to identify firms that are most efficiently using quality management practices to achieve superior quality and innovation performance. The author is not aware of any research to-date that has sought to identify such firms and their practices. As with multicollinearity, the research methods that have been used in existing studies have been suited to 
testing theory and establishing relationships rather than identifying best practices. Exceptions have been two studies discussed previously that used case studies ([82, 100]); however again, the emphasis on these studies was not explicitly in the identification of best practices.

In summary, organizations today must manage for quality and innovation simultaneously; quality remains a key driver of competitiveness and innovation is widely seen as the next competitive frontier. Much of the existing literature does not fully account for the multidimensional nature of both quality and innovation and does not address approaches to implementing TQM (e.g., implementation company-wide and proactive customer focus) that appears to have potential to lead to enhanced performance, particularly with respect to innovation. Further, there is a need to identify benchmark organizations from which managers can learn best practices for simultaneous achievement of quality and innovation. 


\section{Chapter 3. Research Methods}

The primary research question is: What are the best practices in quality management that lead to quality and innovation performance? More specifically, three propositions are presented based on the review of literature on quality and innovation. These propositions address the TQM dualities described previously, i.e., how varying emphases and extent of implementation of TQM impact performance. Specifically:

- Proposition 1: Firms that emphasize a combination of hard and soft factors of TQM outperform (with respect to quality and innovation performance) firms that emphasize primarily the hard factors.

The definitions of 'hard' and 'soft' developed and tested by Lewis et al. [48] were used to determine varying emphases in firms studied here. A firm may provide evidence of emphasis by being able to describe in some detail their approach to a specific factor such as how and where they use analytical tools related to process management (i.e., hard tools) or their approach to obtaining customer feedback (a soft factor). Details of how these factors were assessed are described below.

- Proposition 2: Firms that implement TQM companywide outperform (with respect to quality and innovation performance) firms that have a more narrow focus.

Evans and Lindsay [2] and McAdam [23] provide detail on how this distinction may be evaluated. In particular, a companywide implementation of TQM includes emphasis on all three of the fundamental principles of TQM as presented by Evans \& Lindsay: process focus/continuous improvement, customer focus, and participation \& teamwork (Figure 2). In short, absence of 
evidence of emphasis on any of the three principles, suggests a narrower focus and conversely, evidence of emphasis on all three principles suggests a wider focus. As with proposition 1, a firm may provide evidence of emphasis on any of the principles by being able to describe their approach to specific practices within the principle. Said another way, a firm might discuss at length their approach to process management via statistical monitoring tools. At the same time, if the firm were unable to provide examples of practices related to customer feedback and/or satisfaction as well as how employees participate in decision-making, this would serve as one piece of evidence that the firm had a narrow focus (process focus, in this case) for their TQM efforts.

- Proposition 3: Firms that have both an internal and an external focus outperform (with respect to quality and innovation performance) firms with primarily an internal focus.

As stated previously, internal vs. external focus manifests itself in how broadly a firm envisions its context and its approach to customers. In short, a company with an internal focus sees the firm as a closed system. The primary concerns are related to efficiency, continuous improvement, and participation and teamwork; customers are seen as solely those entities that purchase the firm's products. As described by Sitkin et al., an internally-focused firm seeks to satisfy existing customers and is reactive with respect to customer needs [24]. By contrast, a firm with an external focus views the firm as an open system; customers are more broadly defined as 'stakeholders' and include not merely those entities that purchase the firm's products, but also end consumers, the local community, and the environment. Firm that are more externally 
focused proactively seek to educate customers about latent needs and strive to reach new customers. As with the two previous propositions, evidence to support whether a firm has an internal focus or both internal and external, can be found in how the firm talks about its practices. For example, in response to a question such as "how do you obtain feedback from customers?", an internally-focused firm may either be unable to answer the question or might state something suggesting that they primarily respond to complaints. By contrast, an externally-focused firm will be able to describe a proactive process for maintaining contact with customers.

Figure 7 is a flow diagram for steps taken to address the research questions above. Details of each step are described below. 


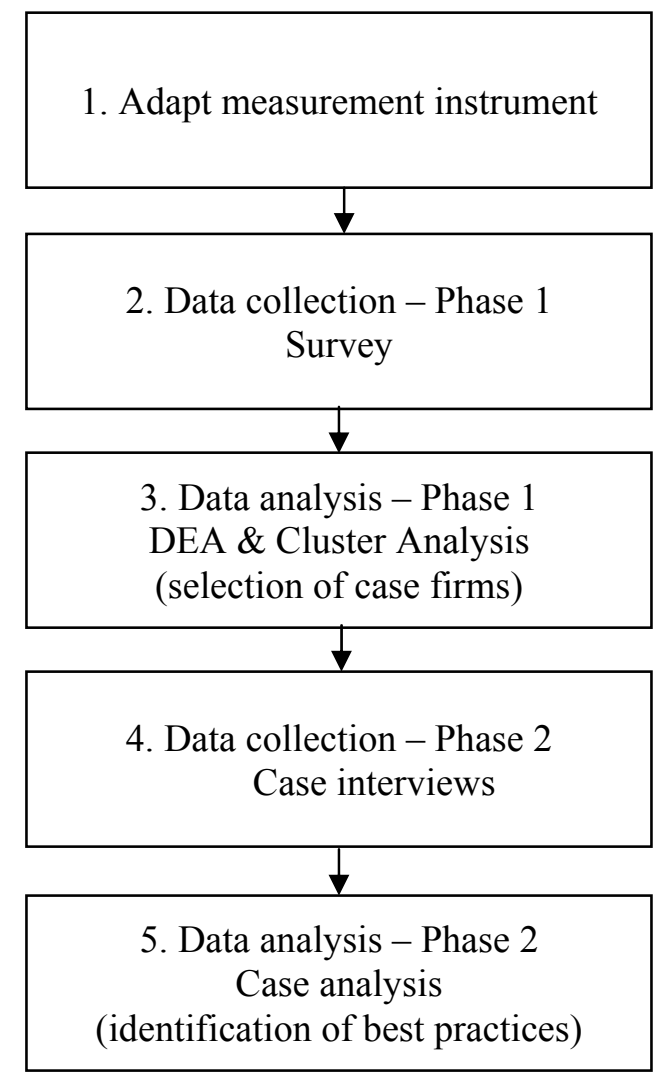

Figure 7. Flow Diagram for Research

\section{$\underline{1 . \text { Adapt measurement instrument }}$}

An existing measurement instrument that has been tested and validated was adapted to measure both 'inputs' (extent of implementation of TQM) and 'outputs' (quality and innovation performance). Details of the measurement instrument are described below.

Extent of implementation of TQM in a firm and performance (quality and innovation) were measured via the instrument developed and used by Prajogo and Sohal [35]; these authors in turn adapted the instrument originally developed and 
tested by Samson and Terziovski [46], discussed previously and shown in Appendix A5.

The Prajogo and Sohal instrument was selected based on the fact that it has been tested and found to be reliable and valid (construct, content, and criterion); in addition, the instrument has recently been used by other researchers exploring the relationships between quality and innovation [20, 106].

Samson and Terziovski developed the scale for measuring extent of TQM implementation based on the Malcolm Baldrige National Quality Award (MBNQA) criteria. However, Prajogo and Sohal adapted the 'Information \& Analysis' section of the Samson and Terziovski instrument because their scale only addressed benchmarking and the MBNQA criteria cover wider issues than benchmarking. Therefore, in addition to benchmarking, questions on 'the availability of data and information about performance', and 'the use of information in decision-making processes conducted by senior management' were included as well.

The performance section of the measurement instrument includes metrics for both quality and innovation. For quality performance, Prajogo et al. [25] reported using the instrument developed by Ahire et al. [50] (see section 11 of Appendix A3). This instrument measures quality as a single construct, however multiple dimensions of quality are included within the construct. 
By contrast, and in keeping with prior research on the topic, innovation is measured by three separate constructs - product, process, and business systems innovation. The Prajogo and Sohal instrument addresses product and process innovation performance, but not business systems/ administrative innovation. Therefore, for the research described here, three questions developed by Johannessen et al. [76] (see Appendix B1) were added to the survey instrument to measure business systems innovation.

In summary, the survey instrument developed and used by Prajogo and Sohal was used nearly verbatim. The only changes made were the addition of three questions on business systems innovation and a question on the respondent's job title and number of years with the organization. The final instrument used is shown in Appendix A6.

\section{Data collection - Phase 1: Survey}

Given the need for a high-level perspective of the firm, target respondents for the survey were senior managers. The target industry sector was the forest industry on the West Coast of the U.S. (California, Washington, and Oregon). This industry and region are desirable given the author's experience, familiarity, and current work responsibilities for research and outreach directed to innovation in the forest industry. The region was limited to the three West Coast states in order to minimize travel costs during case study interviews. Firms with 10 or more employees were included in the survey. Such small firms were included given the author's over 15 years of 
experience in working with this industry sector; while one may not assume that firms with only 10-50 employees would have the resources (financial and personnel) to pursue a formal quality program and/or innovation, the author's experience suggests otherwise, hence, the inclusion of these small firms. In addition, other researchers exploring innovation in the forest industry have explored firms this size and even smaller; for example, Hovgaard and Hansen studied firms with one to 60 employees $[52]$.

The forest industry, broadly defined, includes:

- Forest Products Manufacturing firms, including:

o Structures (and prefabricated structural components) - wood truss manufacturers and prefabricated building manufacturers (e.g., mobile homes and $\log$ homes);

o Primary - sawmills and veneer/ plywood mills (i.e., firms that buy $\operatorname{logs})$;

o Composite and engineered products - (1) firms that use residues from other firms to produce products such as particleboard, (2) firms that use veneer to produce laminated veneer lumber (LVL), or (3) that convert low-value logs to chips or flakes to produce products such as oriented strand board (OSB);

o Secondary - firms that buy lumber, plywood, and composite products to produce furniture, cabinets, moulding/ millwork, doors, windows, etc. and

- Equipment manufacturers (i.e., vendors to the forest industry) that manufacture logging machinery, sawmill machinery, computer-controlled routers, machine vision systems, etc.

The convention in the forest industry is to consider pulp and paper manufacturing as separate and distinct from other sectors of the forest industry. Thus pulp and paper firms were not included in the target sector. 
Research has revealed little innovation, particularly product innovation, in forest products manufacturing. For example, Hansen et al. state, "Very little is understood about how forest sector firms approach new product development or, on the other hand, why many apparently do not proactively develop new products" [101]. Similarly, in a study of the Spanish wood products industry, Diaz-Balteiro et al. state that there is a "...low firm priority towards $\mathrm{R} \& \mathrm{D}$ as a means to achieve competitiveness and an innovation strategy followed by many Spanish firms based on the acquisition of embodied technology available in international markets" [81]. Thus, there was some concern that survey results for forest products manufacturing firms alone might provide little useful information on product innovation. Even for process innovation, there is a greater likelihood that innovation would be accomplished via adoption of purchased technology rather than via development of in-house technologies. This is not surprising given that much of the forest products industry can be considered a mature industry, i.e., in Stage III using the categories developed by Utterback and Abernathy and discussed previously [78]. On the other hand, and in agreement with the literature on innovation in mature industries, equipment vendors to the industry may provide greater opportunity for witnessing depth and breadth in innovation performance. Hence, broadly defining the industry was intended to result in more variation in responses with respect to outputs (particularly innovation performance) as well as inputs, with the assumption that firms take a contingency approach to implementing TQM. 
Questionnaires were mailed to all identified forest industry firms in the three target states using several databases:

- Manufacturers - the author's on-line database of the wood products industry (www.orforestdirectory.com) was combined with a purchased database from USADATA; duplicate entries were removed;

- Equipment vendors - Wood Machinery Manufacturers of America (WMMA); Wood \& Wood Products magazine's 'Red Book' (directory)'; and the Woodworking Machinery Industry Association

For the purchased database, Standard Industrial Classification (SIC) codes included:

- 24 Lumber and Wood Products, Except Furniture

- 2511 Wood Household Furniture

- 2521 Wood Office Furniture

- 25310101 Blackboards, wood

- 25310202 Communion tables, wood

- 2541 Wood Partitions and Fixtures

- 35310103 Logging equipment

- 3553 Woodworking Machinery

The final sample frame included 3305 firms. A hard copy survey and cover letter (Appendix A7) were mailed to each firm and a second mailing was sent to all non-respondents three weeks after the first mailing. Respondents were given the option of responding by self-addressed stamped envelope, by fax, or via a web-based form. Questionnaire responses were collected and tallied in an Excel spreadsheet for subsequent analysis. Non-response bias was assessed using the 'last respondent' method described by Armstrong and Overton [107]. In a mail survey with more than one 'wave' (i.e., separate mailings), this method assumes non-respondents are more 
like the last respondents in the final mailing than the earlier respondents. In this regard, the first 30 respondents (first mailing) were compared to the last 30 respondents (second mailing). Mean responses for all questions were compared using a t-test for samples with independent means. Non-response bias is confirmed in the event there is no statistically significant difference between the 'early' respondents and 'late' respondents.

\section{Data analysis - Phase 1: DEA and cluster analysis}

The primary objective for this step was to select firms for case study interviews. DEA combined with cluster analysis served to identify the firms efficiently using TQM to achieve quality and/or innovation performance outcomes and cluster analysis was used to group the efficient firms based on survey responses to quality and innovation performance. Specifically, the combination of DEA and cluster analysis was intended to identify efficient (i.e., 'high-performing') firms in two diverse categories - those that were achieving primarily quality performance and those achieving both quality and innovation performance. This approach was intended to address the suggestion by Eisenhardt of selecting cases that fill "theoretical categories and provide examples of polar types" [86]. Such a method of selecting case examples is intended to generate wider variety in the responses. Further, results can be compared and contrasted to determine the extent that practices are truly different between the two groups. This distinction between firms is discussed in more detail below. 
$D E A$

Survey responses were analyzed using the input-output model shown in Figure 8. Inputs and outputs for the model were the average of the survey responses for each input and output from each respondent firm (or decision making unit - DMU, to use the terminology of DEA); this method has been used by one group of researchers using similar methodology [38].

\begin{tabular}{|l|}
\hline \multicolumn{1}{|c|}{ Inputs } \\
$\mathrm{X}_{1}$ : Leadership \\
$\mathrm{X}_{2}$ : People Management \\
$\mathrm{X}_{3}$ : Customer Focus \\
$\mathrm{X}_{4}$ : Strategic Planning \\
$\mathrm{X}_{5}$ : Process Management \\
$\mathrm{X}_{6}$ : Information \& Analysis \\
\hline
\end{tabular}

\begin{tabular}{|l|}
\multicolumn{1}{c|}{ Outputs } \\
Quality \\
$\mathrm{Y}_{1}:$ Product performance, reliability, \\
conformance to specifications, \\
durability \\
Innovation \\
$\mathrm{Y}_{2}:$ Product \\
$\mathrm{Y}_{3}:$ Process \\
$\mathrm{Y}_{4}:$ Business systems
\end{tabular}

\section{Figure 8. DEA Input-Output Model Used in this Study}

The specific model used was an output-oriented, constant returns-to-scale (CRS) model as shown previously in Equation 2 [108]. Efficiency Measurement System (EMS) software version 1.3.0 was used to conduct the DEA [109].

The choice of the output-oriented vs. input-oriented model was based primarily on consideration of which model was best aligned with the concept of 'best practices.' For example, were an input-oriented model used, recommendations to managers in inefficient firms would be to seek to use fewer inputs to achieve the existing level of performance (outputs). As a hypothetical example, an inefficient firm with low levels of performance with respect to innovation would in essence be told to maintain poor 
(or mediocre) performance, but to 'scale back' their efforts in, employee empowerment, for example. In short, the message is 'do less' (or invest less effort/resources in current practices).

By contrast, when using an output-oriented model, the recommendation to a manager in an inefficient firm would be to improve performance given existing inputs. As another hypothetical example, an inefficient firm with low levels of performance with respect to innovation would be told to 'scale up' their performance given their current investments in education and training, for example. In short, the message is 'achieve more' (or focus on higher performance).

In the end, neither approach is perfectly aligned with the concept of best practices where the message is 'do something different as a means to achieve higher performance.' However, it was deemed that the output-oriented model was better aligned with the concept than was the input-oriented model.

DEA allows for the identification of efficient and inefficient firms. Firms identified as efficient using the DEA model shown are those that maximize their outputs (quality and innovation performance) while at the same time minimizing their use of inputs (quality management practices). In short, the efficient firms demonstrate focus - emphasis on some practices, lack of emphasis on others. Given the primary research focus on identifying best practices, DEA is used here in order to maximize the potential for identifying differences in practices between case firms. That is, 
without some way of screening candidate firms, there is the potential for examining firms that simply took a 'shotgun approach' to quality management practices. In such a situation there is the potential for practices being more similar than different between firms being studied. In short, regardless of the technique used, the main objective is to somehow reduce the pool of candidate firms to those that demonstrate some diversity in their application of the practices while simultaneously achieving high performance with respect to the specified outputs. DEA is uniquely suited to such an objective.

Within the categories of efficient and inefficient firms, there are sub-categories as well, such as:

1. Inefficient firms:

a. the least efficient DMUs overall,

b. Firms somewhere 'in the middle' (i.e., neither on the efficiency frontier nor in the group above),

2. Efficient firms:

a. DMUs that are efficient due to innovation performance but not quality performance (i.e., "innovation-oriented")

b. DMUs that are efficient due to quality performance but not innovation performance (i.e., "quality-oriented")

c. DMUs that are efficient due to both quality and innovation performance (i.e., "balanced")

For purposes of the research proposed here, groups $2 \mathrm{~b}$ and $2 \mathrm{c}$ represent the groups of greatest interest as these groups allow for differentiation of practices of efficient firms (those effectively using TQM) based on a focus on quality and/or innovation performance. In particular, the "quality-oriented" firms are those that are reaping quality benefits from a quality management system but that presumably have not adapted their practices to achieve innovation performance as well. By contrast, the 
balanced firms are presumed to have made such an adaptation to their practices. Figure 9 shows conceptually the contrast between these firms using the results of DEA.

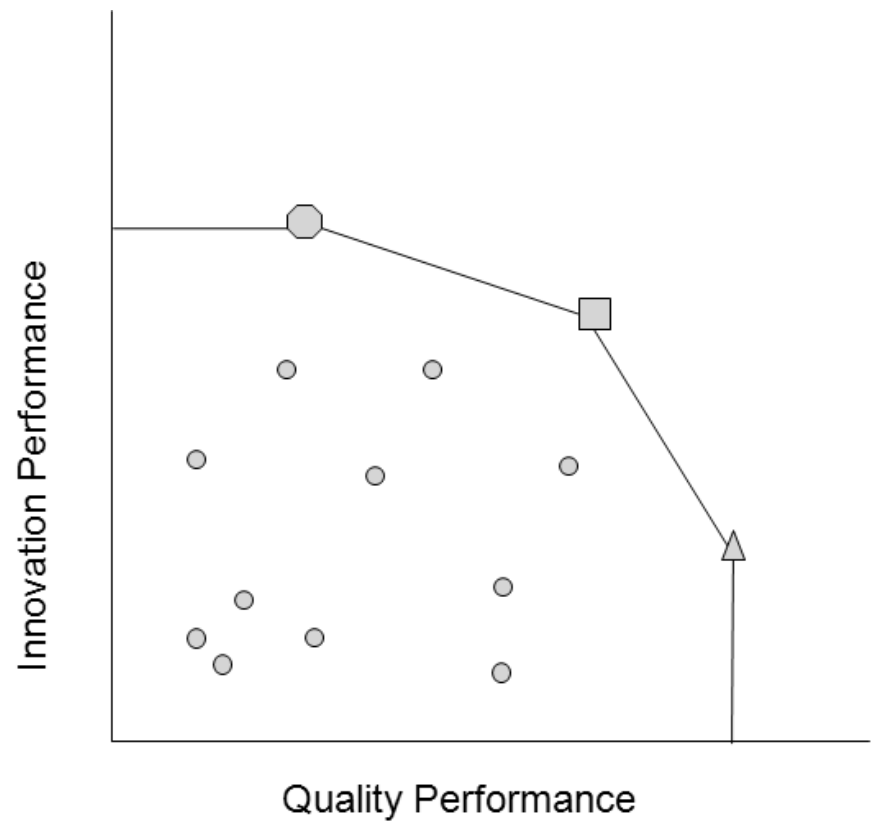

Figure 9. Selection of Cases

The square on the line ('efficiency frontier') in Figure 9 shows a 'balanced' firm; this firm is deemed efficient due to quality and innovation performance (group $2 \mathrm{c}$ described above). By comparison, the octagon represents a firm that is efficient, though primarily due to innovation performance but not quality performance (group 2a described above). For purposes of comparison to the balanced firm, the firm represented by the triangle, the 'quality-oriented' firm is of the highest interest (group $2 \mathrm{~b}$ described above). This firm is efficient due primarily to quality performance but not innovation performance. 
Unfortunately, DEA results do not lend themselves readily to such a categorization of the efficient firms. While the results provide an efficiency score for each firm as well as weights for each input and output, the efficiency scores are unique solutions whereas the weights are not (i.e., reproducible if the linear program were run on another computer). For this reason, cluster analysis was used to categorize the firms into balanced and quality-oriented firms; as stated in the literature, a similar approach to selecting case firms was used by Campbell and Ahrens [91].

\section{Cluster Analysis}

PASW Statistics 18 software (SPSS, 2010) was used to perform the cluster analysis. The specific form of cluster analysis used was hierarchical cluster analysis using the between groups linkage method to form clusters of relatively homogeneous groups; squared Euclidean distance was used as the interval measure. Variables selected for the cluster analysis were the individual output responses from the survey, i.e., the responses to survey questions on quality performance (questions 30-33 in Appendix A6), product innovation (questions 34-38), process innovation (questions 39-42) and business systems innovation (questions 43-45). The primary output of the cluster analysis was a dendrogram, a plot which groups firms with similar values of the selected variables. In this case, the dendrogram clustered efficient firms (as identified previously using DEA) based on how they responded to survey questions on quality and innovation performance. 
"Quality-oriented" and "balanced" clusters were identified (i.e., the clusters were 'named') by examining the average quality and innovation performance responses in the questionnaire for the firms shown in the dendrogram. 'Balanced' firms were determined to be those with an average of five on a five-point scale for quality performance and four or higher on innovation overall; these firms were grouped at the top of the dendrogram. 'Quality-oriented firms' were determined to be those firms with an average questionnaire response of five for quality performance and 2.1 or lower for innovation; these firms were at the bottom of the chart.

The original goal for the research was to select three firms in each category for a total of six firms for case studies. However, the cluster analysis revealed only two candidates in the quality-oriented category. And while there were three candidate 'balanced' firms, one of the firms stated that they were no longer a manufacturer but simply served as a wholesale bark mulch supplier as a result of the economic downturn. Hence, as with the quality-oriented firms, only two firms remained as viable candidates for case interviews. As a result, there were four rather than six firms selected for case interviews. Clearly, a larger number of cases is desirable. However, the use of four or fewer case firms for a research project has precedence in the literature. In fact, two of the articles discussed in the literature review were based on research at a single case firm; Prajogo and Sohal present the results of a study examining one firm in Australia [82] and Bossink examined a single home-building project in the Netherlands [100]. In particular, in one of the most cited papers related to case study research, Eisenhardt states, “... while there is no ideal number of cases, a 
number between four and 10 cases usually works well" [86]. Also, in his textbook on case study research, Yin merely states, “....if multiple candidates are qualified to serve as cases, the larger the number you can study, the better." [88].

\section{Data Collection - Phase 2: Case interviews}

While the DEA analysis of survey responses revealed firms 'efficiently converting TQM inputs into quality and innovation outputs', and cluster analysis allowed for identification of efficient firms that were balanced vs. quality-oriented, more detailed information beyond the survey responses was needed to be able to determine specific differences in practices between the two categories of firms. To get such detail, in-person interviews at each case study firm were conducted. Further, interview questions related to quality and innovation performance served to validate the categorization of the firms. For example, if properly classified, balanced firms should be able to provide evidence of their focus on quality as well as innovation outputs (new products developed, new process technologies developed or adopted, etc.). By contrast, the quality-oriented firms would be expected to provide evidence of their focus on quality but not innovation.

Interview questions were developed with the intent to explore each of the management practices (e.g., Leadership, People Management, Customer Focus) and performance in greater detail as well as to address the three propositions presented previously. To ensure questions also addressed potential areas of difference between the two categories of firms, survey responses of the candidate case firms were 
examined to identify these differences. For example, the firms had similar responses to the question related to Leadership, "Senior executives share similar beliefs about the future direction of this organization"; all four candidate firms ranked this question a four or a five on a five-point scale. However, there were apparent differences in questions related to People Management; for example, balanced firms gave a score of three and four, and both quality-oriented firms gave a score of one for the question "We have an organization-wide training and development process, including career path planning, for all our employees." As another example, balanced firms ranked question 15 on Customer Focus, "We systematically and regularly measure customer satisfaction" at three and four whereas quality-oriented firms ranked this question a two or one.

Draft interview questions were developed and reviewed by two of the author's colleagues (other faculty members at Oregon State University) with experience in case study research. The author's colleagues reviewed the questions for clarity and provided suggestions for improvement. One primary suggestion was to ask 'how' rather than 'yes/no' questions. For example, "how does your company train employees?" rather than "do you have a formal training program?" Interview questions were revised based on these suggestions. Revised questions were then reviewed by an expert panel of five forest industry professionals. The panel provided suggestions such as simplifying the wording of specific questions, rewording others that experts felt were somewhat 'leading', and ensuring respondents that results would 
be confidential. The interview questions were again revised based on suggestions from the expert panel. The final interview questions are shown in Appendix A8.

The four case interviews were conducted in-person at each company. In each case, the interviewee was the same person as responded to the questionnaire. Interviews lasted between approximately 60 and 140 minutes. Interviews were recorded via digital audio recorder (with the interviewee's permission) and then transcribed verbatim. The results were approximately 75 pages of single-spaced transcripts.

In addition, Internet searches were conducted to locate additional information on each case firm. This information was used to complement the case information from the questionnaire and interviews. Each page on the websites of the two balanced firms was copied and pasted into a text document for analysis (described below). In addition, there was another website that discussed the products of one of the balanced firms; data were cut-and-paste into a text document from this site as well. However, no additional information was located for the two quality-oriented firms.

\section{Data Analysis - Phase 2: Case Analysis}

Case study data (survey responses, interview transcripts, and additional information located on the Internet) were input into NVivo software (QSR International, version 8,2009 ) and analyzed following the guidelines suggested by Eisenhardt [86]. The first four of these steps are not involved with data analysis per 
se, but rather establishing propositions, selecting cases, developing measurement instruments, and collecting data. As such, the steps were described previously. However, actions taken within these steps are repeated here for the sake of completeness. The steps in case study research include:

1. Getting started - describe the factors and guiding propositions (expected relationships) among them and propose research questions. The literature review on quality and innovation and measurement instrument presented the factors. In addition, the literature led to the three propositions listed above related to the "TQM dualities", i.e., use of hard vs. soft tools, scope of implementation, and internal vs. external focus.

2. Selecting cases - significant effort has been made here to avoid researcher bias in case selection. Cases were selected that represented balanced firms - those efficiently using TQM to achieve quality and innovation performance - as well as quality-oriented firms - those efficiently using TQM to achieve primarily quality performance. In particular, DEA followed by cluster analysis was used to select the cases. This approach is in keeping with Eisenhardt's suggestion to use 'theoretical sampling', rather than random sampling. That is, cases should be selected to replicate previous cases, extend emergent theory, represent theoretical categories, or polar opposites; multiple cases are preferred to provide deeper understanding and the chance to test propositions. 
3. Crafting instruments and protocols - details are provided above on the development of the survey instrument to measure extent of TQM implementation and performance as well as the interview questions.

4. Entering the field - visits to case firms to conduct interviews were described above. In keeping with the recommendations, analysis occurred simultaneously with data collection via addressing questions such as "what am I learning?" or "how does this case differ from the last?" Eisenhardt states that the researcher is 'encouraged to make adjustments to measurement instruments and methods during data collection.' While no explicit changes were made to the interview questions (i.e., none of the interview questions were reworded), clarifying questions were asked and occasional follow-up questions based on emergent themes developing during the interviews.

5. Analyzing data - the primary approach here is to 'code' the data to compare and contrast within and between the different cases and sources (e.g., survey responses, interview transcripts, and web-based information). As an example of coding, one interviewee responded to the question on benchmarking, "Heck no! I don't care what my competitors do!" This was simply coded under 'benchmarking.' However, as all the cases were coded, cultural differences between firms became apparent which were termed 'proactive/strategic' and 'reactive/conservative.' In this case, the quote above was coded as 'reactive/conservative.' 
Triangulation among the different sources discovers the extent to which there is convergence; data were analyzed and coded within-case first, followed by cross-case. And as recognized by case researchers in the literature, the process is not linear, but iterative. This was the case in this research as well in that emergent themes during coding resulted in the development of new codes and thus several cycles back through the data. The analysis proceeded as follows:

Within-case - transcripts and other data from each case firm were read through line by line four times - the first time as they were being transcribed and then three additional times during coding. For the interview transcripts, responses to questions were coded by the theme of the question; for the management practices, the themes included People Management (communication, employee empowerment, employee satisfaction, training in general, and cross training), Customer Focus (coded as such), and Information \& Analysis (performance measures and benchmarking). Themes related to Process Management were primarily an indication of the extent of usage of the 'hard' quality tools and thus addressed Proposition 1 described above. Details on the coding to address the three research propositions are discussed below.

For the additional (web-based) information, transcribed information was reviewed for themes. For the most part, the web-based information was coded with regards to the output measures of quality and innovation 
performance as this is what companies tend to advertise in their communications to potential customers.

Interview questions on Leadership and Strategic Planning were difficult to code in the first round; the responses were more indicative of company culture rather than specific management practices. In fact, responses to questions on Leadership and Strategic Planning (as well as all the responses, for that matter) led to the development of emergent themes related to company culture, as will be discussed in more detail below. Of course, the themes (e.g., employee empowerment, customer focus, etc.) were not limited to the individual questions on those topics. That is, interviewees often discussed issues related to 'customer focus' in the specific questions on that subject as well as in several other questions.

In addition to coding for the input measures (management practices), within-case analysis also involved coding for the output measures - Quality, Product Innovation (including the approach to new product development), Process Innovation, and Business Systems Innovation. The coding of these output measures also served to validate the categorization of the case firms. For example, if identified correctly, balanced firms should emphasize the importance of quality as well as provide examples of innovations they have either adopted or developed. By contrast, the quality-oriented case firms 
would be similar with respect to their apparent emphases on quality, but should not have examples of innovations.

As described above, the process of coding each case led to the identification of 'emergent' themes. The general nature of emergent themes is that they are implicit in the responses rather than an explicit answer to a question on a topic. For example, although no interview questions directly addressed the importance of relationships, most companies discussed this topic in response to other questions. Further, as discussed above, many responses provided an indication of aspects of company culture. Specifically, some firms were risk-averse and reacted to changes in the market (e.g., "competitors were doing it, so we figured we better get into it too...”). By contrast, other firms discussed risks they had taken and were more proactive in general (e.g., "And I did a lot of research on my own just saying 'hey, can we apply this?'”). Therefore, after the first round of coding, each case was read through a second time and coded for the emergent themes - 'relationships', 'proactive/strategic' and 'reactive/conservative.'

With regards to the three propositions described above, coding was conducted with a specific intent to address these propositions. Comments related to propositions were coded as follows:

- Proposition 1 ('hard vs. soft' factors of TQM) - only in select instances were there essentially 'yes/no' responses that would enable coding on 
the hard vs. soft factors. For example, question 12 in the interview asked which of a list of tools (all of which were 'hard' factors) a company used. A 'yes' answer to this question, combined with details on where and how a tool or tools were used, served as one piece of evidence of a firm's emphasis on hard tools. By contrast, most other instances of hard vs. soft factor emphasis were implicit in a response to a question such, "How do you obtain input/feedback from customers?" A detailed response to this question would serve as evidence of a firm's emphasis on this specific soft factor. By contrast, inability to provide details served as evidence of lack of emphasis on this factor. In particular, interview questions related to People Management, Customer Focus, Process Management, and Information \& Analysis were identified as being the primary sources for evidence (or lack of) of soft or hard factor emphasis. At the same time, ALL questions had the potential to serve as evidence for this proposition depending on the comments of the interviewee.

- Proposition 2(TQM implementation companywide or more narrowly) - As stated previously, absence of evidence of emphasis on any of the three foundational principles of TQM (customer focus, process focus/continuous improvement, and participation \& teamwork), suggests a narrower focus and conversely, evidence of emphasis on all three principles suggests a wider focus. Comments related to this proposition were more difficult to code than for Proposition 1 above. It 
was assumed that directly asking 'is your TQM program implemented companywide?' might be considered a leading question. That is, respondents might answer 'yes' believing that was the 'correct' answer. Therefore, as with Proposition 1, a firm may provide evidence of emphasis on any of the principles by being able to describe their approach to specific practices within the principle. In particular, interview questions related to People Management, Customer Focus, and Process Management were identified as being the primary sources for evidence for this proposition; questions in these three areas represent the foundational TQM principles of participation \& teamwork, customer focus, and process focus/continuous improvement, respectively. As with Proposition 1, however, all interview questions had the potential to serve as evidence for this proposition.

- Proposition 3 (internal vs. external focus) - As stated previously, internal vs. external focus manifests itself in how broadly a firm envisions its context (i.e., a closed vs. open system) and its approach to customers (existing and new). As with the two previous propositions, evidence to support whether a firm has an internal focus or both internal and external, can be found in how the firm talks about its practices. Key interview questions for this proposition included questions related to Customer Focus, Process Management, and Information \& Analysis (the question on benchmarking in particular). In addition, many of the questions related to innovation are likely to 
evoke comments suggesting whether a company is focused on existing customers or new customers. In addition, company websites may provide indications of whether or not a firm is focused on stakeholders beyond direct customers. Lastly, other coding related to 'reactive/conservative' and 'proactive/strategic', to the extent comments applied to the firms' approach to customers, also served as evidence for this proposition.

It should be noted here that one modification to Eisenhardt's recommendations for this step of data analysis was related to development of detailed case study reports for each case. While coding was conducted in this step and summary observations noted for each case firm, the additional step of developing detailed case reports was deemed unnecessary in that the primary objective of determination of best practices sought to establish differences between firms; hence, cross-case analysis and enfolding the literature were emphasized in establishing the chain of evidence rather than within-case analysis.

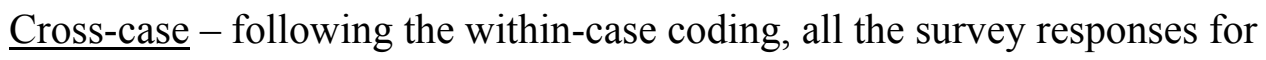
each case firm were examined again ${ }^{3}$ to identify key areas of similarities and differences between case firms. Interview responses were then examined for

\footnotetext{
${ }^{3}$ The questionnaire responses were examined the first time to develop the interview questions.
} 
each coding theme (e.g., 'benchmarking', 'employee empowerment', etc.) to explore similarities and differences between case firms. For example, the qualitative research software allows for combining all the quotes related to any individual theme such as 'customer focus.' These documents were reviewed and notes made related to similarities and differences. It was during this process that the main practices (and cultural) differences between balanced and quality-oriented firms began to emerge. As stated by Eisenhardt, the key feature for this stage of the analysis is the 'chain of evidence.' For this research, the first 'link in the chain' is the company's response to the survey. For example, a company might have rated their practices related to 'employee empowerment' very high. This is evidence of commitment to this practice; however additional evidence, and detail, are needed to validate this questionnaire response. The next, and most compelling, 'links in the chain' on this theme are direct quotes from interviewees related to the topic. Other links in the chain included data from other sources such as websites that are related to the theme. To the extent that multiple sources (questionnaire responses and interview responses) agree, there is a 'chain of evidence' supporting the use of the practice.

6. Shaping propositions - as with the previous steps, the process here was iterative. The initial propositions were compared with the results of the withinand cross-case analysis and new propositions emerged. The process of proposition formation and analysis continued until there was a close fit with the data; each case served as replication in that each case served to confirm or 
disconfirm the proposition. As stated by Eisenhardt, these processes serve to sharpen the definition, validity, and measurability of the constructs and build internal validity.

7. Enfolding literature - given the typically small number of cases, comparison of results with existing literature is done to ensure reliability and validity of findings; generalizability of results occurs when findings are confirmed by a broad range of literature on the topic. To complete this portion of the case research, the literature review was examined in detail and compared to the findings from the within- and cross-case analyses. Notes were made in the literature review when a case firm provided confirming or disconfirming evidence from the literature. For example, Prajogo and Sohal suggested the possibility of 'cross-fertilization' between quality and process innovation performance [25]. Practices at the balanced case firms confirm this statement; quotes from the coding on 'quality' and 'process innovation' are presented to support this point.

8. Reaching closure - this stage focuses on deciding when to stop iterating between data collection and theory development; the 'stop point' is usually when theoretical saturation occurs, i.e., when incremental improvement to the theory is minimal; reporting results as a series of answers to open-ended questions is a good approach. This stage of the research was modified in that the 'stop point' was predetermined given that the number of case firms and interviewees per firm (one) were determined a priori. Therefore, the concept of 'theoretical saturation' was determined on the basis of additional iterations 
through the existing data rather than on collecting additional data (e.g., by

adding case firms and/or additional interviewees at each case firm).

$\underline{\text { Summary }}$

In summary, the research model tested proposes a direct link between six TQM inputs and four outputs that address multidimensional measures of both quality and innovation. A five-step process (shown in Figure 7) was used to address the primary research questions related to best quality management practices for achieving quality and innovation performance; in addition, three propositions were proposed that address the impact of TQM implementation (hard/ soft tool usage; narrow/ broad scope of implementation; and internal/ external focus) on performance. The steps were:

1. Adapt measurement instrument - a quality management measurement instrument developed by Prajogo and Sohal [5] (see Appendix A5) was used to acquire the inputs (quality management practices) and most of the outputs (quality, product, and process innovation performance) for the research model. Three questions developed by Johannessen et al. were added to the instrument to measure business systems innovation [76].

2. Data collection Phase 1 - The measurement instrument was sent to West Coast US firms in the forest industry. Responses were self-reported, perceptual data on each firm's extent of use of quality management principles (inputs) and quality and innovation performance (outputs).

3. Data analysis Phase $1-$ DEA was used to analyze the data and to identify a set of efficient firms, i.e., firms that are efficiently using their investment in TQM principles and practices to generate quality and/or innovation performance outputs. Survey responses related to outputs for the efficient firms were analyzed using cluster analysis to enable identification of firms primarily focused on quality performance (quality-oriented) and firms focused on both quality and innovation performance (balanced). Two firms in each category were identified for case studies. 
4. Data collection Phase 2 - survey responses from each case firm were examined to facilitate development of interview questions. Questions were developed and reviewed by two academic experts in survey research and then pilot tested with five industry experts. Interview questions were revised based on their feedback. In-person interviews were conducted at each of the four case firms; interviews were audio recorded and transcribed verbatim. In addition, Internet searches were used to locate additional information on each firm.

5. Data analysis Phase 2 - all information (interview transcripts and web-based information) was stored in NVivo software and coded following standard qualitative research methods for within and cross-case analysis, and in particular, closely following the steps outlined by Eisenhardt for building theories from case study research [86]. Areas for which there was a chain of evidence (as established during cross-case analysis and via comparisons with existing literature) suggesting differences between the balanced and qualityoriented firms were reported with those practices of the balanced firms indicated as the best practices for quality management to achieve quality and innovation performance. 


\section{Chapter 4. Results \& Discussion}

Results from the research are presented below following the steps of the research - 1. Adapt measurement instrument; 2. Data collection Phase 1 -survey; 3. Data analysis Phase 1 - DEA followed by cluster analysis (selection of case firms); 4. Data collection Phase 2 - case interviews; 5. Data analysis Phase 2 - case analysis (identification of best practices). The chapter concludes with a summary of the key findings.

\section{$\underline{\text { Measurement Instrument }}$}

Results for this portion of the research were discussed in the previous section. However the information is briefly summarized here for sake of completion. An existing measurement instrument that had been tested and validated was adapted to measure both 'inputs' (extent of implementation of TQM) and 'outputs' (quality and innovation performance). Extent of implementation of TQM in a firm and performance (quality, product and process innovation) were measured via the instrument developed, tested, and used by Prajogo and Sohal [5] (see Appendix A5). Three questions developed by Johannessen et al. were added to measure business systems innovation performance [76]. The measurement instrument used in the research is shown in Appendix A6. 


\section{Data Collection Phase 1 - Survey}

The survey was mailed to 3305 firms and a second mailing was sent to nonrespondents after three weeks. Participants were given the option to respond by hard copy (via postage paid envelope), fax, or by a web-based version of the survey.

A total of 298 surveys were returned as undeliverable and 146 were identified as duplicates (surveys sent to the same company). Fifty-two firms returned the survey saying it was not applicable to their firm, or that they had either closed, moved (outside the three-state study region), or retired. Finally, post-survey examination of the firms revealed an additional 155 non-respondent firms that were either closed or not in the target industry sector (e.g., logging contractors, forestry consultants, or retail establishments). Hence, the adjusted sample frame included 2654 firms.

There were 215 total respondents; 137 firms responded to the first mailing and 78 to the second. The majority of the questionnaires were returned via US mail - only 10 firms responded using the web-based survey and four by fax. Lastly, 11 respondents were eliminated due to incomplete sections of the survey (e.g., none of the questions on leadership were answered, or quality performance, etc.). Hence the response rate was 7.7 percent $(204 / 2654)$.

Regarding sample size, for DEA, the heuristic established by Banker et al. suggests that the number of decision making units (DMUs) should be greater than 
three times the sum of the number of inputs and outputs [110]. Thus, in a model with six inputs and four outputs a minimum of 30 DMUs are needed. Hence, from the standpoint of DEA, the sample size is more than adequate. In addition, the number of respondents was similar to that of the research discussed in the literature review exploring the impact of TQM on performance - between 150 and 200; however, the response rate in the present research is considerably lower.

\section{Non-response Bias}

Results of the t-test for non-response bias are shown in Appendix D. The test supports the absence of a response bias $(p>0.05)$ for 44 of the 45 mail survey questions. The one exception was question 44 related to 'ability to obtain new sources of supply.' For this question, the t-test comparing responses of the first 30 respondents to the last 30 respondents suggests that non-respondents may be less able to obtain new sources of supply (95 percent confidence interval for difference in means $[0.12-1.10])$. Such bias is not thought to significantly impact the results of the research in that both quality-oriented and balanced firms reported success in this area, as is discussed below.

Respondents by Industry Sector and Number of Employees

The sample population was categorized into five industry sectors, as described previously:

- Structures

- Primary manufacturers 
- Composite and engineered products

- Secondary manufacturers

- Equipment manufacturers

A Chi-square goodness of fit test was conducted to assess how the industry sector represented by the respondents compared with that in the sample population.

Results are shown in Table 7.

Table 7. Respondents by Industry Sector

\begin{tabular}{|l|l|l|l|l|l|}
\hline $\begin{array}{l}\text { Industry } \\
\text { Sector }\end{array}$ & Population & Respondents & Expected & Chi-Square & $\begin{array}{l}\text { Significance } \\
(1 \text {-tailed })\end{array}$ \\
\hline Structures & 188 & 15 & 14.50 & 0.02 & 0.991 \\
\hline Primary & 703 & 84 & 54.22 & 16.36 & $<0.001$ \\
\hline Composites & 186 & 24 & 14.35 & 6.50 & 0.04 \\
\hline Secondary & 1502 & 73 & 115.84 & 15.85 & $<0.001$ \\
\hline Equipment & 66 & 8 & 5.09 & 1.66 & 0.435 \\
\hline Total & $2645^{*}$ & 204 & & & \\
\hline
\end{tabular}

*Industry sector is unknown for nine firms.

Results indicate that there is a significant difference $(p<0.05)$ between the population of interest and the respondents with regards to the primary, composites, and secondary manufacturing sectors. Specifically, there were significantly more respondents than expected for primary manufacturers and fewer than expected for both composites and secondary manufacturers. As a result, the research findings may not be generalizable within the forest industry in general, let alone to other industries. In particular, results are more likely to be representative of manufacturers of structures, primary wood products, and equipment, but perhaps not composites or secondary wood products manufacturers. 
A Chi-square goodness of fit test was also conducted with respect to firm size (number of employees). Results are shown in Table 8 . As can be seen, the target population is composed of predominantly small firms. Results indicate there were significantly more respondents than expected for firms with more than 100 employees. The findings may therefore be more representative of larger, rather than smaller firms.

Table 8. Number of Employees in Population vs. Survey Respondents

\begin{tabular}{|l|r|r|r|r|r|}
\hline $\begin{array}{l}\text { \# of } \\
\text { Employees }\end{array}$ & Population & Respondents & Expected & $\begin{array}{r}\text { Chi- } \\
\text { square }\end{array}$ & $\begin{array}{r}\text { Significance } \\
\text { (1-tailed) }\end{array}$ \\
\hline $10-100$ & 2201 & 143 & 167.37 & 3.55 & 0.17 \\
\hline $100-500$ & 313 & 42 & 23.80 & 13.91 & $<0.001$ \\
\hline $500+$ & 24 & 8 & 1.83 & 20.89 & $<0.001$ \\
\hline Total & $2538^{*}$ & 193 & & & \\
\hline
\end{tabular}

*Number of employees was not provided for 116 firms (and 11 respondents)

However, there are other factors that influence generalizability of the findings. According to Eisenhardt, generalizability for case research occurs when findings are confirmed by a broad range of literature on the topic [86]. Hence, while industry sectors and firm size represented by the survey respondents may not fully represent the population of interest, findings may still be generalizable within and beyond the industry studied to the extent that findings are confirmed by other research. Correspondence of the findings with prior research is discussed below in the 'enfolding the literature' phase of Data Analysis Phase 2.

\section{Data Analysis Phase 1 - DEA and Cluster Analysis}

DEA resulted in identification of 26 'efficient' firms. Efficiency scores and weights resulting from the analysis are shown in Appendix E. The dendrogram 
resulting from the cluster analysis is shown in Figure 10 below. As discussed above, the cluster analysis served to identify efficient firms who rated themselves high on both quality and innovation performance ('balanced' firms) and those who rated themselves high primarily on quality but not innovation ('quality-oriented' firms). As can be seen from the dendrogram, there are three firms (DMUs 2740, 1235, and 1833) in the cluster near the middle that have average quality scores of five (on a five-point scale) and the highest average innovation scores (all above four) of all the efficient firms. Therefore, these three firms were deemed the most appropriate candidates for balanced firms.

By contrast, there are two firms (DMUs 2967 and 385) that also have average quality scores of five; however these firms average innovation scores are below 2.2. And while there are four firms that have lower innovation scores (e.g., DMUs 143, 1485,1543 , and 1508) none of these firms combine this low innovation score with an average quality score of five. For this reason, only firms 2967 and 385 were selected as candidates for quality-oriented firms.

Each of these five firms (three balanced and two quality-oriented) firms were contacted and asked to participate in an interview. Two of the three balanced firms (DMUs 1235 and 1833) agreed to participate in an interview. As discussed previously, the third firm (DMU 2740) declined to participate in that the company was no longer a manufacturer. The two balanced firms are highlighted with a square in 
Figure 10. Both quality-oriented candidate firms (DMUs 385 and 2967) agreed to participate; these firms are highlighted with an oval in Figure 10.

\section{Dendrogram using Average Linkage (Between Groups)}

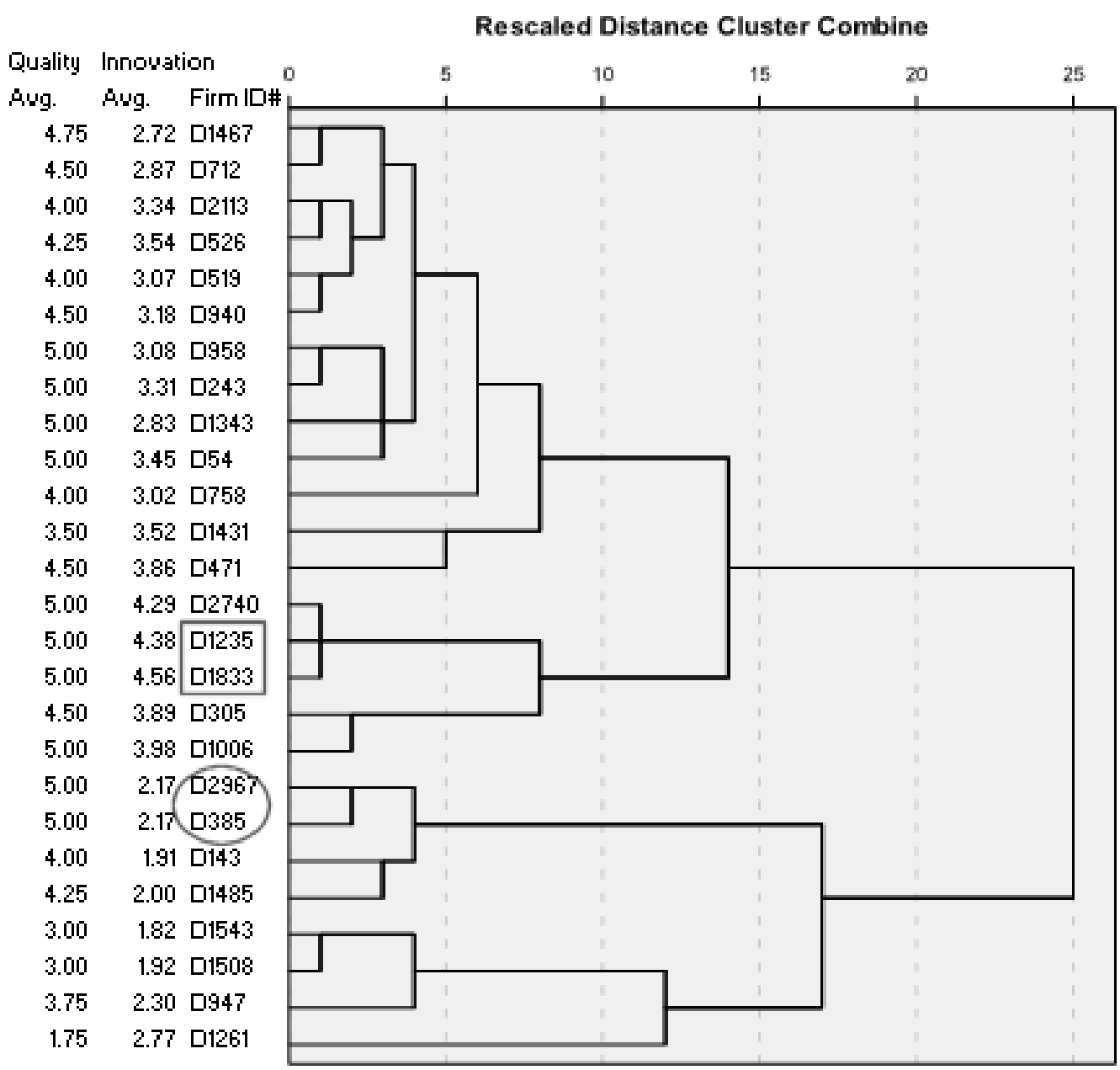

Figure 10. Cluster Analyis Dendrogram and Average Performance Responses for DEA-Efficient Firms

Table 9 shows the characteristics of the case firms and interviewees at each firm. 
Table 9. Characteristics of Case Firms

\begin{tabular}{|l|l|l|}
\hline & $\begin{array}{l}\text { Balanced } \\
\left(\text { Case firm } \mathrm{B}_{1}, \text { Case firm } \mathrm{B}_{2}\right)\end{array}$ & $\begin{array}{l}\text { Quality-Oriented } \\
\left(\text { Case firm } \mathrm{QO}_{1}, \text { Case firm } \mathrm{QO}_{2}\right)\end{array}$ \\
\hline Job title & Vice president, co-owner & President/owner, President \\
\hline Years w/ company & 5,4 & 21,25 \\
\hline Employees & $\sim 75,10-15$ & $\sim 50,10-15$ \\
\hline Industry sector & Lumber, cabinets & Remanufacturing, trusses \\
\hline State & Oregon, Washington & $\begin{array}{l}\text { Oregon \& Washington } \\
\text { (mill in OR, office in WA), } \\
\text { California }\end{array}$ \\
\hline
\end{tabular}

As can be seen from the table, the goal of interviewing senior managers was attained - all interviewees had job titles of owner, vice president, or president. It is also interesting to note the differences in years with the company for interviewees at balanced firms vs. quality-oriented firms. While interviewees were not asked their age, the author estimates that all were approximately 50 to 60 years of age. As such, the balanced firm representatives were not 'young and inexperienced' managers per se but, based on interview comments, an individual that was with a relatively new firm in one case and an individual that was new to a well-established firm in the other case.

The case firms were small companies - all four firms had 75 or fewer employees. Further, there was one relatively small company (10-15 employees) and one slightly larger company (50-75 employees) in each category. Thus, the potential for bias between balanced and quality-oriented firms based on company size is not as likely as it would be if there were, for example, large companies in one category and small companies in the other. Further, the fact that all four case firms had fewer than 75 employees appears to correspond with the target population well. That is, given that the vast majority of the firms in this industry sector are small companies, the fact 
that the case firms are also small companies is desirable from the standpoint of generalizability of research findings to this industry sector.

From the standpoint of industry sectors, three of the five industry sectors are represented by the case firms - primary (lumber and remanufacturing), secondary (cabinets), and structures (trusses). The fact that none of the case firms represent composite products and equipment may limit the generalizability of the findings.

Lastly, from the standpoint of representation of West Coast U.S. states, as can be seen from the table, two of the case firms' mills were located in Oregon (1 of which had its sales office in Washington), one of the case firms was located in Washington, and the other in California. Therefore, even with a small sample number of case firms, each of the West Coast states from the sample population is represented.

\section{Data Collection Phase 2 - Case Interviews}

As discussed above, interview questions were developed with the intent to explore each of the management practices (e.g., Leadership, People Management, Customer Focus) and performance in greater detail, to address the three propositions presented previously, and to validate the categorization of the firms. To ensure questions also addressed potential areas of difference between the two categories of firms, survey responses of the candidate case firms were examined to identify these differences. For example, there were apparent differences in questions related to 
People Management; balanced firms gave a score of three and four, and qualityoriented firms gave a score of one for the question - "We have an organization-wide training and development process, including career path planning, for all our employees."

Draft interview questions were developed and reviewed by two of the author's colleagues (other faculty members at Oregon State University) with experience in case study research. Interview questions were revised based on their suggestions. Revised questions were then reviewed by an expert panel of five forest industry experts. The interview questions were again revised based on suggestions from the expert panel.

The four case interviews were conducted in-person at each company with the person that responded to the questionnaire. Interviews were recorded via digital audio recorder and then transcribed verbatim. In addition, Internet searches were conducted to locate additional information on each case firm. Each page on the websites of the two balanced firms was copied and pasted into a text document. No additional information was located for the two quality-oriented firms.

With respect to categorization of the firms (balanced vs. quality-oriented) quotes from interviews with the firms and web-based information for the balanced firms provide evidence that the case firms were properly categorized. 
With respect to quality, all four firms were expected to be able to provide evidence of focus on quality. Examples of quotes from interviews related to quality include:

"So I think that the quality again, is in the eyes... the beauty is in the eyes of the beholder, it's in the eyes of the consumer."

Case firm $\mathrm{QO}_{1}$

"Quality control is just something that the business was built on..." Case firm $\mathrm{QO}_{2}$

"I mean we are very very quality conscious and when we're putting out the best product in the game, you've got to be the best."

Case firm $\mathrm{B}_{1}$

"If anybody is eyeing quality and not just low-bid, I think we are by far the best value."

Case firm $\mathrm{B}_{2}$

Web-based information for the balanced firms provides additional evidence of the firms' emphasis on quality:

"to further ensure the quality of the product, [product trade name] are checked for quality, grade and moisture content before and after they are dried"

Case firm $B_{1}$ (from $3^{\text {rd }}$ party website)

"[Company name] set themselves apart from the competition with quality, craftsmanship \& service."

Case firm $\mathrm{B}_{2}$ (from company's website)

As expected, the balanced firms also provided evidence of innovation performance. Examples of quotes from interviews include:

"We spent a lot of money so far developing a two-head CNC machine that will automatically put that look on." 
Case firm $\mathrm{B}_{1}$ - response to question about new product development; indicative of process innovation as a prerequisite for product innovation.

"We started out with a 1-1/8 [inch] exterior grade particleboard and then we bonded a backer sheet and a top laminate sheet; we used the type two water resistant PVA, polyvinyl acetate glue, and tried to make it as waterproof as possible."

Case firm $\mathrm{B}_{2}$ - discussing new product development to ensure product durability.

Websites for the balanced firms provide additional evidence of the firms'

emphasis on quality and/or innovation:

"Our dedication to using the latest technologies to improve our products and processes makes us a market leader in high quality [product line]. With innovations such as [product trade name 1] and [product trade name 2] we lead the high-quality market with products and service."

Case firm $\mathrm{B}_{1}$ (from company website)

"[company name] uses their [process trade name] to produce the finest quality dry timbers on the market. The [technology] is the only method..."

Case firm B1 (from forest industry trade magazine website). Note: most of the quote cannot be shown without violating confidentiality.

"Using exterior-grade board and water-resistant glue provides an added measure of long-term durability rarely found in plastic laminate counters."

Case firm $\mathrm{B}_{2}$ (from company website) - discussion of product innovations for the purpose of ensuring product quality (durability).

"...field measurements are taken, relevant appliance specs are received and the job is laid out in our [software]. Computer renderings are provided to the customer/ builder/designer/ architect for review and revision, as necessary. Once the layout has been finalized... machine codes are created and sent over the computer network to the shop floor for production."

Case firm $\mathrm{B}_{2}$ (from company website)

In addition, the balanced firms appeared to view innovation as a means to an end. For example, the quotes above indicate these firms pursued product innovation 
as a means to improving product quality or process innovation to improve efficiency and/or as a prerequisite for product innovation.

In contrast, the quality-oriented firms provided evidence of a lack of focus and/or interest in innovation. Examples of quotes from interviews include:

"I think our actual technology is probably behind our competitors. You know from the standpoint of degree of automation it probably is... So our kind of belief is why let yourself fall into a trap like that? When it's not a proven technology - in other words, technology is wonderful when it's proven."

Case firm $\mathrm{QO}_{1}$ - response to question about the processing technology the firm uses

“We really don't. Outside of, like I say if there's stuff other companies are providing that our customers are asking about... There's nothing that we have developed along those lines. It's the industry standard type stuff."

Case firm $\mathrm{QO}_{2}$ - response to question about how new products are developed

Given that the two quality-oriented firms did not have websites, no additional information is available for these firms beyond their responses to the questionnaire and interviews. In fact, the fact that the firms do not have websites may serve as additional evidence of their lack of emphasis on innovation (business systems innovation in particular). Lastly, and in contrast to the balanced firms, the qualityoriented firms appeared to view innovation simply as 'technology' rather than as a means to some other goal. The statement, "technology is wonderful - when it's proven" made by firm $\mathrm{QO}_{1}$ captures this sentiment well.

Data Analysis Phase 2-Case Analysis

As described in the chapter on Research Methods, case study data (survey responses, interview transcripts, and additional information located on the Internet) 
were analyzed following the guidelines suggested by Eisenhardt [86]. Specifically the steps taken included:

- Analyzing Data - examining and coding data within and between cases;

- Shaping Propositions - further analyzing the initial propositions and proposing and exploring new propositions that emerged during the analysis (for internal validity);

- Enfolding Literature - comparing findings with existing literature (for external validity/generalizability); and

- Reaching closure - determining when to stop collecting data and presenting results.

Results are presented below following each of these steps.

\section{Data Analysis: Within-Case}

Within-case analysis involved reading through the interview transcripts and web-based information several times and coding comments by theme. For example, specific quotes of interest within the answer to the question "Describe the modes of communication in your company..." were coded under the theme 'communication.' However, when the interviewee discussed aspects related to communication in responses to other questions, those quotes were coded under 'communication' as well. The primary intent of the within-case analysis was to code the data in preparation for the cross-case analysis that followed, including coding related to the three propositions. In addition, during the coding process, general observations (e.g., phrases that were repeated, indications of aspects of company culture, etc.) were made about each of the firms. 
Rather than present detailed information on the coding for each firm, general observations are presented here for each of the case firms. More in-depth analysis is given below in the section on cross-case data analysis; results of the analysis related to the propositions are discussed in the section on Shaping Propositions that follows. Quality-Oriented Firm $1\left(\mathrm{QO}_{1}\right)$ :

- Leadership - senior managers' role is "... accurately defining a business environment today and in five years and being able to integrate the two."

- 'Hands-on management' - the interviewee said this phrase nine times during the interview. This company president firmly believes in being directly involved in all operations of his business. For example, he worked on the production floor two days a week. As a corollary, the view on employees was “...I don't believe in employees doing their own thing."

- People vs. technology - the interviewee stated that companies should “...put your time and money and effort in your people rather than a computer." However, other comments such as the comment above related to employee empowerment demonstrated that the primary driver here was not the value placed on the individual but rather that during a down market you have to keep making payments on 'technology' whereas employees can be let go.

- Reactive/market-driven - the primary focus was on reacting to what the market was doing rather than trying to drive the market (i.e., market-driven vs. market-driving). For example, the preference was to use existing processing capacity to react to market opportunities rather than taking the risk to introduce new products. As stated, "... what that comes down to is matching the markets with your technological capacity."

- Risk-averse - With regards to new product development, the view was, "...we'd just as soon pass up that quote unquote opportunity ...let somebody else break trail."

- Quality is defined by the customer - "I think quality is perceived... it is perceived by the customer as to whether or not they buy your product and how much they buy." 
Quality-Oriented Firm $2\left(\mathrm{QO}_{2}\right)$ :

- 'Do it right the first time.' - The interviewee said this phrase four times during the interview (related to strategic planning, process management, and business systems innovation). This was one 'link in the chain of evidence' of the firm's emphasis on quality.

- Emphasis on long-term relationships - with regards to customer satisfaction, the interviewee simply stated, "We have real good relationships with our customers. Word gets back fast." And with respect to suppliers, he told a detailed story about how they maintained a relationship with an individual lumber broker; after the broker was unfairly terminated by the broker's employer, the case firm stayed with the individual in his new employer (i.e., they switched suppliers, in order to maintain the relationship with the same individual broker).

- People vs. technology - as with the other quality-oriented firm, there was an emphasis on employees using well-established (and perhaps outdated) technology and doing things by hand. And just as with the other qualityoriented firm, this was not an indication of the value placed on employees. For example, when asked about employee involvement in a recent change in the company the response was, "they were simply trained to use the technology."

- Reactive/market-driven - as the interviewee stated, "We had all the work we could handle come through the door... of course when things crashed now we're like OK, time to learn how to go out and sell." Similarly, the company avoided one particular product line but began to offer the product because 1) competitors were offering it and 2) the market 'crashed' so they needed the business.

- Quality as defined by the firm (vs. the customer) - aside from 'do it right the first time', the firm also emphasized using the highest quality raw materials, even when competitors were cutting costs by using lower quality material. In that regard, he stated, "I wouldn't put something like that out but they [competitors] do it routinely. And so, customer doesn't know, the average guy doesn't know."

- Customer service - the company had a strong focus on serving the end customer (in their case, the homeowner) rather than just the homebuilder. The 
interviewee told several stories related to how they would find mistakes on drawings brought in by a homeowner (vs. the contractor who was paying for the product).

Balanced Firm $1\left(\mathrm{~B}_{1}\right)$ :

- 'Balance' on several dimensions - there were several aspects where this firm demonstrated balance, beyond quality and innovation performance:

o People and technology - in the question about employee satisfaction, the interviewee told a story about their production manager being frustrated by being unable to keep one of their key process innovations operating at full capacity. As the interviewee stated, keeping the innovation running was, "the most important thing", he also stated, "...my job is balance... to communicate to them that I understand your job, I understand your issues, but here's why it is how it is."

o Quality and customer service - the interviewee relayed the story of a customer complaint - "...out of the 400,000 feet of wood you've bought so far this year you've got 10 pieces with skip [lumber surfacing defect]. We're going to try harder but...it's easier for me to talk to a customer than to go and ratchet down our QC a little bit more. It's that balance..."

o Structure and flexibility - the interviewee described their process improvements and innovations as being very structured and rigorous with the use of advanced statistical tools. However, NPD processes were described as being "loosey-goosey" in that he would simply try things in such a way as to not interfere with production.

- Proactive/market-driving - this company purchased another firm that developed and patented a lumber processing innovation and, then with inhouse efforts had "..been able to double the throughput and reduce the capital costs." Given that the finished product was new to the industry, the firm then worked to train architects (those that specified their products in a structure) about the quality benefits the innovation provided. As such, the firm essentially worked to drive change in the market and thereby generate demand for their product. 
- Risk-takers - the preceding point as well as several others related to process and product development provided evidence that the firm was amenable to taking risk in developing new technologies, entering new markets, etc.

Balanced Firm $2\left(\mathrm{~B}_{2}\right)$ :

- 'Balance' on several dimensions - as with the other balanced firm, this firm demonstrated balance, although the outcomes and focus were different:

o People then technology - like the other balanced firm, the interviewee talked about how they had tried to involve and empower employees and create a family atmosphere (e.g., by celebrating employee birthdays during the lunch hour) but struggled to get good employees. As a result, they invested in processing technology to circumvent the need for skilled, dedicated employees.

o Quality and customer service - the company recognized that many of their customers (contractors) were often too busy to sort through dozens of potential product combinations (wood species, color, finish type, etc.) even while still desiring custom cabinets. So they created four product lines and visited new home construction sites to proactively provide bids.

o Process efficiency and quality - the use of state-of-the art technology enabled both process efficiencies ("you press a button and it sends it out to the CNC and it's nested-based, flat table, it just cuts all the pieces") while also enabling the firm to maintain consistent product quality.

- Proactive/market-driving - the firm felt that durability of existing products (theirs and their competitors') was quite poor. So they developed more durable products, which required process innovations as well. They offered a 10-year warranty - something unheard of in the industry - and then tried to educate customers about the benefits of this product in an effort to drive demand. In short, this was not something customers were asking for but rather a product innovation they introduced to the market. 
- Risk-takers - several of the examples above are evidence of the firm being amenable to risk. One key example is in their proactively providing drawings and bids to contractors. As the interviewee acknowledged, they were assuming a risk here by providing non-copyrighted drawings that could be used by competitors to offer counter-bids.

As can be seen from even this brief summary of each of the case firms, some themes are beginning to emerge related to similarities and difference between the firms. These distinctions are explored in greater detail below in the cross-case analysis.

\section{Data Analysis: Cross-Case}

Results are presented here first by areas of similarity between the categories of firms. Similarities in questionnaire responses are presented first followed by quotes from interviews that either serve to confirm or disconfirm the questionnaire responses. This comparison of questionnaire responses with interview comments is one aspect of triangulation - where multiple sources of evidence converge to either confirm or disconfirm findings.

The cross-case analysis concludes with a presentation of areas that were either mixed or different, again beginning with questionnaire responses and concluding with quotes from interviews. Table 10 presents the cross-case summary of questionnaire responses with respect to management practices (inputs) and Table 11 presents a similar summary for performance (outputs). 
Table 10. Cross-Case Comparison of Questionnaire Responses (Inputs)

\begin{tabular}{|c|c|c|}
\hline $\begin{array}{l}\text { Theme/ } \\
\text { Principle } \\
\text { (Inputs) }\end{array}$ & $\begin{array}{l}\text { Questions w/Similar Responses } \\
\left(\mathrm{B}_{1}, \mathrm{~B}_{2} / \mathrm{QO}_{1}, \mathrm{QO}_{2} \text { responses }\right)\end{array}$ & $\begin{array}{l}\text { Questions w/Mixed or Different Responses } \\
\left(\mathrm{B}_{1}, \mathrm{~B}_{2} / \mathrm{QO}_{1}, \mathrm{QO}_{2} \text { responses }\right)\end{array}$ \\
\hline Leadership & $\begin{array}{l}\text { Execs. share similar views on future } \\
\text { direction }(4,5 / 5,4) \\
\text { Managers encourage change and culture of } \\
\text { improvement }(4,5 / 4,4)\end{array}$ & $\begin{array}{l}\text { Employees have opportunities to share in } \\
\text { change }(4,4 / 2,4) \\
\text { Unity of purpose, elimination of barriers } \\
(3,5 / 4,4)\end{array}$ \\
\hline $\begin{array}{l}\text { People } \\
\text { Management }\end{array}$ & $\begin{array}{l}\text { Measurement of employee satisfaction } \\
(2,1 / 1,1) \\
\text { Employee flexibility \& multi-skilling } \\
(3,3 / 3,4) \\
\text { Healthy \& safe work environment }(5,4 / 5,5)\end{array}$ & $\begin{array}{l}\text { Organization-wide training \& development } \\
(4,3 / 1,1) \\
\text { Top-down \& bottom-up communication } \\
(2,4 / 4,3)\end{array}$ \\
\hline $\begin{array}{l}\text { Customer } \\
\text { Focus }\end{array}$ & $\begin{array}{l}\text { Close relationships } w / \text { customers }(5,5 / 5,5) \\
\text { Effective process for resolving complaints } \\
(5,4 / 5,5)\end{array}$ & $\begin{array}{l}\text { Actively seek customer inputs }(5,2 / 4,3) \\
\text { Customer needs disseminated }(4,5 / 3,3) \\
\text { Involve customers in product design } \\
(4,5 / 1,4) \\
\text { Measurement of customer satisfaction } \\
(4,3 / 1,2)\end{array}$ \\
\hline $\begin{array}{l}\text { Strategic } \\
\text { Planning }\end{array}$ & $\begin{array}{l}\text { Structured planning process, long \& short- } \\
\text { term goals }(2,2 / 1,1) \\
\text { Incorporate needs of stakeholders }(2,1 / 1,1) \\
\text { Written statement of strategy }(2,1 / 1,1)\end{array}$ & Mission statement $(3,2 / 1,1)$ \\
\hline $\begin{array}{l}\text { Process } \\
\text { Management }\end{array}$ & $\begin{array}{l}\text { Standard operating procedures }(2,2 / 2,3) \\
\text { Use of statistical techniques }(1,1 / 1,2) \\
\text { Use of supplier rating system }(1,1 / 1,1)\end{array}$ & $\begin{array}{l}\text { Fool-proof processes }(4,4 / 5,3) \\
\text { Concept of internal customer }(3,5 / 1,1) \\
\text { Long-term relationships w/suppliers } \\
(3,5 / 5,5)\end{array}$ \\
\hline $\begin{array}{l}\text { Information } \\
\& \text { Analysis }\end{array}$ & $\begin{array}{l}\text { Performance measurement system } \\
(1,1 / 1,2) \\
\text { Use of benchmarking }(2,1 / 1,2)\end{array}$ & $\begin{array}{l}\text { Avail. up-to-date data on perf. }(2,2 / 4,1) \\
\text { Review of perf. for decision-making } \\
(2,4 / 1,4)\end{array}$ \\
\hline
\end{tabular}

Table 11. Cross-Case Comparison of Questionnaire Responses (Outputs)

\begin{tabular}{|l|l|l|}
\hline $\begin{array}{c}\text { Theme/ } \\
\text { Principle } \\
\text { (Outputs) }\end{array}$ & \multicolumn{1}{|c|}{$\begin{array}{c}\text { Questions w/Similar Responses } \\
\left(\mathrm{B}_{1}, \mathrm{~B}_{2} / \mathrm{QO}_{1}, \mathrm{QO}_{2} \text { responses }\right)\end{array}$} & $\begin{array}{l}\text { Questions w/Mixed or Different Responses } \\
\left(\mathrm{B}_{1}, \mathrm{~B}_{2} / \mathrm{QO}_{1}, \mathrm{QO}_{2} \text { responses }\right)\end{array}$ \\
\hline Quality & $\begin{array}{c}\text { Product performance }(5,5 / 5,5) \\
\text { “ } \quad \begin{array}{c}\text { conformance }(5,5 / 5,5) \\
\text { reliability }(5,5 / 5,5) \\
\text { durability }(5,5 / 5,5)\end{array}\end{array}$ & $\begin{array}{l}\text { Level of newness }(5,5 / 2,3) \\
\text { Use of tech. innovations in prod. }(5,5 / 1,3) \\
\text { NPD speed }(4,5 / 2,2) \\
\text { No. new products introduced }(4,5 / 2,1) \\
\text { No. new products } 1 \text { st to market }(4,5 / 1,1)\end{array}$ \\
\hline $\begin{array}{l}\text { Product } \\
\text { Innovation }\end{array}$ & & $\begin{array}{l}\text { Tech. competitiveness of co. }(5,5 / 1,2) \\
\text { Speed of adopting tech. innov. }(5,5 / 1,1) \\
\text { Level of newness of tech. }(5,5 / 1,1) \\
\text { Rate of change in process/techniques/tech. } \\
(4,5 / 2,2)\end{array}$ \\
\hline Innocess & & Ability to penetrate new markets $(4,3 / 2,2)$ \\
& & \\
\hline $\begin{array}{l}\text { Business } \\
\text { Systems } \\
\text { Innovation }\end{array}$ & $\begin{array}{l}\text { Ability to obtain new sources of supply } \\
(4,4 / 5,4)\end{array}$ & $\begin{array}{l}\text { Success in seeking new ways to org. } \\
\text { business }(4,4 / 4,3)\end{array}$ \\
\hline
\end{tabular}


Similarities between Firms - Management Practices

As shown in Table 10, there is no broad management practice for which both categories of firms responded similarly to all the questions. In general questions related to Leadership were rated relatively high (three to 5) and either similar or mixed. The interviews appear to disconfirm the quality-oriented firms' responses to the question about 'managers encouraging a culture of change and improvement...' This could be a case of a poorly-worded question in that multiple concepts were embedded in a single question. That is, it is possible that these managers rated themselves highly on a culture of improvement; and there was some evidence of that fact, e.g., "do it right the first time". However there was no evidence of encouraging change or a culture of improvement in these two firms.

For People Management, the case firms all rated themselves quite low on measuring employee satisfaction; interviews confirmed this fact. However, there were apparent differences in how firms viewed the importance of employee satisfaction (regardless of whether or not it was actually measured). For example:

"We don't assess employee satisfaction. They either like it or don't like it." Case firm $\mathrm{QO}_{1}$

"We communicate well and we're hoping in this market anyway that job satisfaction goes a long way. And job satisfaction is being open and heard and respected and all the things that anybody would want.... and knowing when someone's frustration is actually resulting in lost production, in lost opportunity, in loss of quality." Case firm $\mathrm{B}_{1}$

Interviews confirmed the fact that all the firms had some approach to employee flexibility and multi-skilling (i.e., cross-training). 
Lastly with regards to People Management, it is not surprising that all firms rated themselves highly for having a safe and healthy work environment given that this is a key area of concern in manufacturing, wood products manufacturing in particular. Further, one would not expect a senior manager to report on a survey that they did not focus on employee safety and health.

With regards to Customer Focus, the case firms rated themselves highly in having close relationships with customers and in having effective processes for resolving complaints. No questions were asked during the interviews on these topics, however all of the firms made comments about the importance of close relationships with customers and hence there is evidence to validate the respondents' high ratings for this practice.

There was very close agreement in the low scores given for Strategic Planning. Given that all four case firms were small companies (all had 75 or fewer employees), low emphasis in this area is not surprising. In general, the interviews for the two quality-oriented firms confirm these low ratings in that the firms responded to the question about long-term planning with statements such as:

"Which way is the wind blowing? I think what you've got to do is you've got to sit back, take a look at the economic environment that you're in, take a look at the markets you're in, which do you focus on, which do you not focus on." Case firm $\mathrm{QO}_{1}$ "To try and survive, we just cut costs everywhere we can." Case firm $\mathrm{QO}_{2}$ 
However, one of the balanced firms made statements that appear to disconfirm the low rating they gave themselves on the questionnaire for activities related to Strategic Planning:

"...where we want to be in three years, five years, and once we decide, our vision statement now is we want to be the number one place in North America to come to for any type of [product line]. When somebody thinks [product line], we want them to call us first... We focus on facility development, what services and products to provide, market development, developing strategic partnerships with clients,... financial planning, business control procedures.”

Case firm $B_{1}$

Case firm $B_{2}$ was not able to articulate how they approached strategic planning. As such, perhaps their questionnaire responses were accurate. However, the interviewee talked about proactively seeking new customers, developing new products, etc. that demonstrates the firm had an approach that was perhaps more implicit than explicit.

For Process Management, all the case firms rated themselves quite low on the use of standard operating procedures (SOPs), statistical techniques, and a supplier rating system. While no interview questions addressed SOPs or supplier rating systems, there were questions about quality tools, including statistical tools. For three of the firms, the low rating on the questionnaire was confirmed in that interviewees did not use the majority of the quality tools listed such as statistical process control, design of experiments, etc. However, case firm $\mathrm{B}_{1}$ 's response to this question disconfirms the low rating they gave this item:

"And that's why we can get to our operating results we do out of those [equipment] because it's statistics statistics statistics... Statistical Process Control, we do that in 
our QC. We keep track of all sorts of things. Checksheets - when I said that we had fairly strong procedures, once we decide to make a procedure change, and that gets into checksheets. Cause and effect diagrams - not so much for the production, but [manager name] and I use them a lot.... Histograms - pages and pages from all sorts of departments..."

Case firm $\mathrm{B}_{1}$

In general, case firms rated themselves relatively low on management practices related to Information \& Analysis. The use of an effective performance measurement system and benchmarking in particular were rated quite low. Interview responses appear to confirm the lack of emphasis on a performance measurement system. And the quality-oriented firms confirmed their lack of emphasis on benchmarking:

“Absolutely not. I don't care what my competitors do. I don't look at them. I don't think about them. Fifteen years ago I did. And that was a mistake. I think that you do what you do and you run hard."

Case firm $\mathrm{QO}_{1}$

"For the most part when you hear feedback it's after you've done the job and they say well you were \$2000 cheaper than this guy"

Case firm $\mathrm{QO}_{2}$ - interviewee stated that they benchmarked competitors; however quote suggests that at best, the efforts are passive

However, the balanced firms' responses to the interviews suggest that they do in fact benchmark competitors, firm $\mathrm{B}_{1}$ in particular:

"We try and benchmark our uptime for an industrial... for a complex industrial process. We compare very well. We're running around 98.5 percent of total available time in a day those machines are running. So we've got a really really good technology and we find ways to keep it running continually. And so we benchmark ourselves against other industries." Case firm $\mathrm{B}_{1}$ 
“... our main competitor was [company name]...they dial in things better, they have economies of scale, better purchasing, more efficient... if you go into our jobs and our competitor jobs, I think ours stand out. I think almost all the time very few competitors met our quality."

Case firm $\mathrm{B}_{2}$ - suggests knowledge of competitors only obtainable via active benchmarking

\section{Similarities between Firms - Performance}

Given that the two categories of firms were established via cluster analysis on the survey responses related to quality and innovation performance (outputs), most of the similarities and differences shown in Table 11 are to be expected. In particular, the similarity in responses with respect to quality and differences with respect to innovation are to be expected given the definitions of the 'balanced' vs. 'qualityoriented' categories. As stated previously, the interviews confirmed that all four firms were focused on quality.

However, the firms were far more similar than anticipated with respect to two questions related to business systems innovation. Specifically, firms responded similarly on questions related to 'ability to obtain new sources of supply' and their 'success in seeking new ways to organize their business.' Interviews confirmed these responses in that all interviewees were able to describe how they had sought and located new suppliers and how they had reorganized their businesses due to the ongoing recession at the time of the interviews.

In summary, many of the apparent similarities between case firms with respect to quality management practices identified in the examination of questionnaire 
responses were confirmed by the interviews. However, there are a few practices where the interviews served to disconfirm the questionnaire responses. These practices merit further exploration in that they may be added to the list of those in the following section - practices that serve to differentiate between the two categories of firms. These practices are included in the summary of apparent differences in practices in Table 12 at the end of this section.

Differences between Firms - Management Practices

The previous section emphasized areas where the case firms responded $\underline{\text { similarly }}$ to the questionnaire and to interview questions. However, of perhaps greater interest for addressing the research questions here is where the firms were either mixed or differ in their practices. Again, questionnaire responses are examined first, followed by interview data.

With regards to Leadership, questionnaire responses (Table 10) suggest that the results were 'mixed' with respect to the quality-oriented firms' responses to the question about 'employees having opportunities to share in change.' Specifically, $\mathrm{QO}_{1}$ rated themselves a two on this question whereas $\mathrm{QO}_{2}$ rated themselves a four. However, evidence was found to suggest that a much lower rating was warranted for case firm $\mathrm{QO}_{2}$. For example, in response to the interview question of what role employees played in the most recent change the company had experienced, the interviewee stated (without any further elaboration): 
"Employees were simply trained to use the new technology."

Case firm $\mathrm{QO}_{2}$

Similarly, case firm $\mathrm{QO}_{1}$ stated:

"So, the question is to lead change, I think the employees have got to follow my change. That's the role of employees - to get someone that can sit back and will do what you tell them to do and not get fancy."

Case firm $\mathrm{QO}_{1}$

Therefore, it appears that this is in fact a practice where the difference between balanced and quality-oriented firms is more pronounced than was indicated by the questionnaire responses. In contrast, balanced firms addressed this question with responses such as:

"And that's typically how, in our company, we innovate. We rely on our supervisors and our sub-managers and frankly our employees to tweak what's there and to figure out... here we've got this equipment, but if we use it this way we could do better. We encourage them to look at what we've got. How do we make what we've got a little bit better? ...And we really rely on them for that."

Case firm $\mathrm{B}_{1}$

With regards to the Leadership practice of 'unity of purpose, elimination of barriers', results were mixed in that all firms ranked themselves high (four and five) with the exception of $\mathrm{B}_{1}$ that rated their firm a three. Unfortunately, no interview questions were asked on this topic. Therefore differences or similarities in this practice can neither be confirmed nor disconfirmed.

With regards to People Management, balanced firms rated themselves a three or four on the question related to 'organization-wide training \& development' whereas both quality-oriented firms rated themselves one. However, interview comments do 
not appear to confirm this difference. In fact, it appeared that the results were mixed one firm in each category $\left(\mathrm{QO}_{1}\right.$ and $\left.\mathrm{B}_{1}\right)$ provided evidence of organization-wide training whereas the other firms in each category $\left(\mathrm{QO}_{2}\right.$ and $\left.\mathrm{B}_{2}\right)$ did not. For example, the firms that appeared to have a commitment to training stated:

"we do an enormous amount of in-house training. We do an enormous amount of [lumber] grading... So we start teaching everybody and we cross-train by having not just the graders but the graders and cutters will flip-flop constantly so that they're constantly being upgraded because that's such a critical method... for our equipment, we'll send them to different classes that they have on keeping them up-to-speed on that..." Case firm $\mathrm{QO}_{1}$

"There is a formal training program in all the key areas and just typically - they are started off at a fairly low-risk position... to make sure that we're training 2, 3, four other guys in a very deliberate way to keep bringing up their level of knowledge." Case firm $\mathrm{B}_{1}$

Whereas the firms lacking such a commitment stated:

"On-the-job"

Case firm $\mathrm{QO}_{2}$ - This was the answer (without elaboration) to the question, "How are new employees trained?"

"We didn't have a formal training process. Which I know, that's what I always felt we'd try and get somebody trained in certain areas - yeah, all on the job training. The training, I think if we had better structure..."

Case firm $\mathrm{B}_{2}$

Also with regards to People Management, questionnaire responses to the question related to 'top-down and bottom-up communication' were mixed in that case firm $B_{1}$ rated themselves relatively low (two) whereas the others rated themselves a three or four. The interviews did not appear to confirm this assessment by $\mathrm{B}_{1}$. For example, the interviewee stated: 
"They all know that they can come and talk to me and it's not going to go on deaf ears, it's not going to result in me lashing out or something like that. It's OK, let's hear it! And they know that my job is to listen...we flow fairly openly with ideas and communication and all that."

Case firm $\mathrm{B}_{1}$

For Customer Focus, responses were mixed with regards to 'actively seeking customer input.' Case firm $\mathrm{B}_{1}$ rated their firm a five whereas $\mathrm{B}_{2}$ rated themselves a two; $\mathrm{QO}_{1}$ and $\mathrm{QO}_{2}$ rated themselves a four and three respectively. However, the interviews did not appear to confirm this difference between balanced firms in that case firm $\mathrm{B}_{2}$ stated:

"we meet with customers and it's one to two months by the time you meet with them initially, give them a drawing, bids, go back-and-forth, and revisions by the time they get competitive bids."

Case firm $\mathrm{B}_{2}$

In hindsight, it appears that the nature of firm $\mathrm{B}_{2}$ 's business (custom cabinets) was such that 'actively' seeking customer was not needed; products could not be produced without active customer input. Therefore, evidence suggests the case firms are more similar than different with respect to 'actively seeking customer input.' In addition, there was little evidence that the quality-oriented firms actively sought customer input. On the contrary, most evidence suggested these firms relied on customers to provide them input, as confirmed by comments related to 'measuring customer satisfaction' discussed below.

With respect to the questionnaire responses related to 'customer needs disseminated', balanced firms rated their firms higher on this activity than did quality- 
oriented firms. Interview responses appear to confirm this difference in that quality-

oriented firms weren't able to address the practice. In contrast, firm $\mathrm{B}_{1}$ stated:

"It will go first through the account rep to the person that's handling that account, the specific account. And typically it comes through me. And this customer said this this this. If it's just anecdotal or if it's just a one-off frustration, I typically won't get involved. If it's something that I sense is substantial or worthy of communication I'll just talk to the customers directly, I know them all... And if there's things that we can change, communicate that.... [employees hear about these sorts of things] when it's an event or an issue that effects our production."

Case firm $\mathrm{B}_{1}$

However, $\mathrm{B}_{2}$ 's response did not seem to address the question:

"Since we're a custom shop - what kind of wood do you want, do you want alder, cherry, maple, hickory, whatever. So go through that, drawer guides, materials, hinges, interior species of the, and all that, color, go through and get all the stuff, the customers to the showroom, here's all the different alternatives, and it got, so they'd come in - oooh, it's like a kid in a candy shop."

Case firm $\mathrm{B}_{2}$

The implication of this response could be similar to the previous question - as a custom shop, disseminating customer input on the product is simply 'standard operating procedure' as it is required to be able to produce the product. Hence, where firm $B_{1}$ assumed 'customer input/feedback' referred to customer complaints, firm $B_{2}$ assumed it referred to product design details (wood species, color, etc.).

Results were mixed for the question related to 'involving customers in product design.' Firms $\mathrm{B}_{1}, \mathrm{~B}_{2}$, and $\mathrm{QO}_{2}$ rated themselves either a four or five on this question, however $\mathrm{QO}_{1}$ rated their firm a one. Comments from the three firms $\left(\mathrm{B}_{1}, \mathrm{~B}_{2}\right.$, and $\mathrm{QO}_{2}$ ) provide evidence that these firms involve customers in product design.

However, given that the interviewee at $\mathrm{QO}_{1}$ did not address the question directly, it is 
hard to say if his comments support the low rating. At the same time, the response to the question on $R \& D$ provides no evidence of involving customers in design:

"We don't do anything formal. We turn around and take a look and see what's there and take a look and see what's in the marketplace and we try to adapt."

Case firm $\mathrm{QO}_{1}$

Finally, with regards to Customer Focus, with respect to 'measuring customer satisfaction', the balanced firms rated themselves slightly higher (three and four) on this practice than quality-oriented firms (one and two). While none of the firms provided evidence of actually measuring customer satisfaction (e.g., with formal questionnaires), the interviewees did provide evidence of a difference between firms:

"I'll just talk to the customers directly, I know them all." Case firm $\mathrm{B}_{1}$

"A lot of times it is tracking them down trying to not talk to them on the phone so much but meet them face-to-face... If someone did a formal thing on our customers I think they would rate us very high on customer service. I think extremely high. We tried to use the best products and I think we were always very customer oriented." Case firm $\mathrm{B}_{2}$

"It's real simple - if they keep buying from you, they're satisfied."

Case firm $\mathrm{QO}_{1}$

"Word gets back quickly."

Case firm $\mathrm{QO}_{2}$

In addition, the balanced firms' websites (and perhaps the mere fact that they

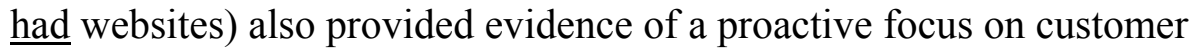
interaction/customer service. For example, $\mathrm{B}_{1}$ 's website includes links to CAD details and specifications for use by architects; the firm also provides an on-line 
continuing education course certified by the American Institute of Architects. Other examples include statements on their websites:

"Custom milling services... allow us to produce the product you need, when you need it. We will pull your order to the piece; we will mill and package to your specifications..."

Case firm $\mathrm{B}_{1}$ - company website

"[company name] has proudly taken customer service to the next level, providing customers with complimentary cabinet drawings WITHOUT copyright restrictions. Potential customers can have [company name] custom design their cabinet layout, and they are free to show our designs to our competitors to get comparison bids..." Case firm $\mathrm{B}_{2}$ - company website

The bottom line for this topic ('measuring customer satisfaction') combined with the findings above for 'actively seeking customer input' appears to be that while none of the firms actually described a process for measuring customer satisfaction, the balanced firms were proactive in their approach to interacting with customers whereas the quality-oriented firms appeared to be far more reactive or passive.

For Strategic Planning, while all firms rated themselves low here, the balanced firms rated having a mission statement at three and two whereas both quality-oriented firms rated themselves a one. Interview comments simply supported the notion that none of the firms placed much emphasis in this area. For example, comments included:

"Not formal ones no. But we know where we want to go."

Case firm $\mathrm{B}_{1}$ - response to question, "Do you have mission and vision statements?"

"I don't really believe in a mission statement. I think the function of a business is real simple - your job is to make money."

Case firm $\mathrm{QO}_{1}$ 
With regards to Process Management, questionnaire responses were mixed with respect to 'fool-proof processes' in that firm $\mathrm{QO}_{2}$ rated their company a three on this practice and the other firms rated themselves four and five. Unfortunately, none of the interview questions directly addressed this practice. Therefore, similarities or differences between firms cannot be confirmed or disconfirmed.

With respect to firms' understanding of the 'concept of the internal customer' in their firm, balanced firms rated their firms either neutral or high (three and five) with regards to the concept, whereas both quality-oriented firms rated their firms a one. Interviews with the quality-oriented firms confirmed their lack of emphasis on this concept. However, the interviews with the balanced firms provided mixed results. In fact, $B_{1}$ that rated their firm a three on the concept provided evidence of understanding of the concept whereas firm $\mathrm{B}_{2}$ that rated their firm a five, did not provide evidence indicating their understanding of the concept:

"the core of our business is our [trade name] technology. And in that aspect, that department very much looks at the rest of the company as their customer. For everything after [trade name], the planing, the detailing, the finishing, the texturing, that aspect is... the customer there are our traders, our salesmen, which are also by extension our customers. So I guess you can say from our production... when you step out of just the drying to our next level, we do have that to where they're serving our traders needs, our salesmen's needs as well as our customers.”

Case firm $\mathrm{B}_{1}$

"...we have better equipment than many companies 10 or 20 times our size. We have good equipment and a high production, high quality... to me a nested base flat table machine makes so much more sense than a beam saw and a point-to-point machine or something like that.”

Case firm $\mathrm{B}_{2}$ - response to question about 'emphasis of internal customer concept' (response suggests lack of understanding of the concept) 
Therefore, at best, it can be said that the differences between firms with regards to the 'concept of the internal customer' are mixed.

With respect to the Process Management practice of 'long-term relationships with suppliers', questionnaire results were mixed in that $\mathrm{B}_{1}$ rated their firm a three and all other case firms rated their firm a five. Again, interview evidence suggested that firm $B_{1}$ may have 'under-rated' their firm on this practice:

“...I can't see us really bringing on new suppliers for awhile. You really want to support the guy that you have to make sure everybody gets through this [recession]. So we've made a conscious decision just to make sure we can send them as much business as we can to help them out." Case firm $\mathrm{B}_{1}$

Therefore, it appears that any differences in practices related to 'long-term relationships with suppliers' that were based on the questionnaire responses are disconfirmed by the interviews.

For Information \& Analysis, results from the questionnaire were again mixed. For the question related to the 'availability of up-to-date information on performance', three of the firms rated themselves a one or two, however one quality-oriented firm rated themselves a four. Unfortunately, the interview questions simply focused on measures of performance rather than explicitly addressing whether or not the measures were 'up-to-date', as stated in the questionnaire. Therefore, additional analysis of this practice is not possible. 
Lastly with respect to Information \& Analysis, practices related to 'review of performance for decision-making', questionnaire results were also mixed. $\mathrm{B}_{1}$ and $\mathrm{QO}_{1}$ rated their firms low (two and one, respectively) while both $\mathrm{B}_{2}$ and $\mathrm{QO}_{2}$ rated their firms four. Unfortunately, this practice is among one of the most difficult to assess the accuracy of the firms' ratings on the questionnaire. Comments related to the practice include:

"Well, first and foremost is profit. And that's the driver. We keep track in the [process trade name], particularly, that's where our biggest capital investment is. We keep all sorts of stats on reliability figures, operating percentages - are we 99 percent uptime this month, 97... so we keep a lot of stats on that because that's where our money is invested."

Case firm $\mathrm{B}_{1}$

"What does this job cost? Did we make a profit?"

Case firm $\mathrm{B}_{2}$

"The bottom line. In the past we have looked at how much volume or board footage we go through. The only thing that counts is the bottom line. That's the only thing." Case firm $\mathrm{QO}_{1}$

"Do we have money in the bank? Are we paying the bills? Again, nothing formal... we've never really done a true assessment of what it actually costs us to do this and do that. They say there's a lot of tools in our software that we could be utilizing." Case firm $\mathrm{QO}_{2}$

The interviews suggest that all the firms rely primarily on the 'bottom line', however firm $B_{1}$ provided some additional detail as well. In short, it seems the firms are more similar than different for this practice.

Differences between Firms - Performance

As stated above, the differences in questionnaire responses between the balanced and quality-oriented firms were largely to be expected given how the 
categories were established (i.e., by cluster analysis) and defined (i.e., quality-focused vs. quality and innovation-focused). The balanced firms rated themselves a four or five for all of the questions related to Product Innovation and Process Innovation whereas the quality-oriented firms rated themselves a one or two for nearly all of the questions. The exceptions were that $\mathrm{QO}_{2}$ rated their firm a three for the 'level of newness of their new products' and their use of 'technological innovations in new products.' However, interview evidence seems to confirm these ratings may be a bit 'generous.' For the former ('level of newness') the interviewee was unable to answer the question 'List some examples of new products you've developed in the last three to five years." And for the latter, the interviewee stated:

"There's nothing that we have developed along those lines. It's the industry standard type stuff."

Case firm $\mathrm{QO}_{2}$

Lastly with respect to differences in innovation performance, with regards to Business Systems Innovation, balanced firms rated their 'ability to penetrate new markets' higher on the questionnaire than did quality-oriented firms. Unfortunately, none of the interview questions explicitly addressed this topic. Therefore, no additional information is available to confirm or disconfirm the apparent difference. Regardless, and as stated above, the questionnaire responses and interviews served to validate the categorization of the firms - balanced firms provided evidence of innovation performance whereas quality-oriented firms did not. 
The results of the cross-case analysis are summarized in Table 12. For each practice, balanced firms placed either greater or similar (shown as 'mixed') emphasis than quality-oriented firms on the quality management practice listed. The table summarizes practices that differ between balanced and quality-oriented firms in that these practices may serve to differentiate between the categories. As such, these practices are presented as an initial set of potential 'best practices' for firms wishing to adapt their quality management practices in order to achieve innovation performance in addition to quality performance. Validating these practices based on findings from existing research may serve to narrow the list.

Also, there were several practices for which the questionnaire responses suggested a difference between firms. However, these differences were not able to be confirmed or disconfirmed in that interview questions did not explicitly address the practices. Therefore, the most that can be said is that there are potential differences in these practices and further examination is suggested. The practices are:

- There is a high degree of unity of purpose in our company, and we have eliminated barriers between individuals and/or departments.

- We design processes in our plant to be 'fool-proof' (preventive-oriented)

- Up-to-date data and information of company performance are always readily available for those who need them.

The first and third points in particular are interesting in that they are the only practices for which quality-oriented firms may have placed higher emphasis than did the balanced firms. 
Table 12. Quality Management Practices that Differ between Balanced and Quality-Oriented Firms

\begin{tabular}{|l|l|}
\hline Theme & Quality Management Practice \\
\hline Leadership & $\begin{array}{l}\text { Senior managers actively encourage change and implement a culture of } \\
\text { improvement, learning, and innovation in pursuit of 'excellence' } \\
\text { Employees have the opportunity to share in and are encouraged to help } \\
\text { the organization implement changes }\end{array}$ \\
\hline People Management & $\begin{array}{l}\text { A We actively work to ensure employee satisfaction } \\
\text { We have an organization-wide training and development process, } \\
\text { including career path planning, for all our employees (mixed) }\end{array}$ \\
\hline Customer Focus & $\begin{array}{l}\text { Customer needs and expectations are effectively disseminated and } \\
\text { understood throughout the workforce }\end{array}$ \\
& $\begin{array}{l}\text { We involve customers in our product design processes (mixed) } \\
\text { B We proactively interact with our customers and seek their input }\end{array}$ \\
\hline Strategic Planning & $\begin{array}{l}\text { We have a comprehensive and structured planning process which } \\
\text { regularly sets and reviews short and long-term goals }\end{array}$ \\
\hline Process Management & $\begin{array}{l}\text { The concept of the 'internal customer' (i.e., the next process down the } \\
\text { line) is well understood in our company (mixed) } \\
\text { We make extensive use of statistical techniques (e.g., SPC) to improve } \\
\text { the processes and to reduce variation (mixed) }\end{array}$ \\
\hline $\begin{array}{l}\text { We are engaged in an active competitive benchmarking program to } \\
\text { measure our performance against the 'best practice' in the industry. }\end{array}$ \\
\hline
\end{tabular}

${ }^{\mathrm{A}}$ This practice was reworded - none of the firms provided evidence of measuring employee satisfaction; however, both balanced firms provided evidence of having some approach to (or simply concern for) ensuring employee satisfaction whereas quality-oriented firms expressed 'disinterest' in the concept. ${ }^{\mathrm{B}}$ This practice was reworded because none of the firms provided evidence of measuring customer satisfaction. However, both balanced firms provided evidence of proactive customer interaction whereas quality-oriented firms' approach appeared to be reactive or passive.

With respect to performance, the findings were for the most part as expected given how the categories of firms were determined and defined: all firms were focused on quality performance and balanced firms were focused on innovation performance as well. In particular, balanced firms were focused on product and process innovation broadly, and business systems innovation with respect to 'ability to penetrate new markets.' However, data were not collected that would allow this latter point to be confirmed. 
The fact that the balanced and quality-oriented firms may be similar with respect to two aspects related to business systems innovation ('ability to obtain new sources of supply' and 'success in seeking new ways to organize their business') was not expected. At the same time, given that raw material supply is one of the key challenges for the forest industry (and something at which all successful firms must become adept) and the fact that the nation was in a recession when this research was being conducted, perhaps it should not be surprising that there were similarities among case firms in these areas.

\section{Shaping Propositions}

Within- and cross-case analysis also included a focus on addressing the three propositions stated in the Research Methods section as well as 'shaping' and examining new propositions that emerged from the data. The initial propositions are repeated here followed by confirming or disconfirming evidence and the conclusions. Finally, a new emergent proposition is stated along with supporting evidence.

- Proposition 1: Firms that emphasize a combination of hard and soft factors of TQM outperform (with respect to quality and innovation performance) firms that emphasize primarily the hard factors.

- Proposition 2: Firms that implement TQM companywide outperform (with respect to quality and innovation performance) firms that have a more narrow focus.

- Proposition 3: Firms that have both an internal and an external focus outperform (with respect to quality and innovation performance) firms with primarily an internal focus. 
Proposition 1 - Hard vs. Soft Factors/Tools

Based on the distinctions between the categories of firms (balanced vs. qualityoriented), this proposition may be restated as:

- Proposition 1 (restated) - balanced firms emphasize both hard and soft factors of TQM whereas quality-oriented firms emphasize primarily hard factors.

The first approach taken to address this proposition was to examine Table 11 (revised with the summary results shown in Table 12) to look for indications that the firms are different with respect to the 'soft' factors (and in particular, that balanced firms place greater emphasis in these practices) and at the same time, that the firms were similar with respect to the 'hard' factors. The first three rows in the table (Leadership, People Management, and Customer Focus) represent the soft factors and the last three rows (Strategic Planning, Process Management, and Information \& Analysis) represent the hard factors. Therefore, strong evidence in support of this proposition would be: 1) the table cells for the soft factors were empty in the "Questions w/ Similar Responses" column and 2) the table cells for the hard factors were empty in the "Questions w/Mixed or Different Responses” column. Statement 1 would provide evidence that balanced firms place greater emphasis on the soft factors and Statement 2, that all firms place similar emphasis on the hard factors.

A limitation of the approach just described is that it assumes the practices are equally important. That is, from the standpoint of impact on quality and/or innovation performance, perhaps the Customer Focus practice of 'actively seeking customer input' should receive twice the 'weight' as having an 'effective process for resolving 
complaints.' Unfortunately, the literature does not provide such a weighting scheme. Therefore, the assumption is made that the practices are equal in importance, with the acknowledgment that this is likely not strictly accurate.

Examination of Table 11 shows that no such clear distinctions existed. In fact, on the contrary, the cross-case analysis shows that there are apparent differences in practices within each of the themes regardless of the 'hard/soft' factor distinction. Hence, this first 'link' in the chain-of-evidence suggests that the proposition is not supported.

A second approach to evaluation of the evidence was stated in the chapter on Research Methods - particular attention was given during coding to practices related to People Management (soft), Customer Focus (soft), Process Management (hard), and Information \& Analysis (hard). However the entire transcripts were coded for statements that reflected emphasis on either a hard or a soft factor. The number of comments that were related to a hard factor and those related to a soft factor were then tallied for each case firm. Many of the quotes in the previous section would serve as examples of such coding. For example, a statement related to the use of statistical process control would be coded as a hard factor and a statement related to efforts made to interact with customers would be coded as a soft factor. Table 13 below shows the results of this tally. 
Table 13. Number of Comments Related to Hard and Soft TQM Factors

\begin{tabular}{|l|l|l|}
\hline Case Firm & Soft & Hard \\
\hline $\mathrm{B}_{1}$ & 33 & 31 \\
\hline $\mathrm{B}_{2}$ & 17 & 22 \\
\hline $\mathrm{QO}_{1}$ & 18 & 15 \\
\hline $\mathrm{QO}_{2}$ & 15 & 10 \\
\hline
\end{tabular}

While no attempts are made to assign statistical significance to the results of such a tally, the primary intent here is to provide another link in the chain-of-evidence for the proposition. In short, if the proposition were confirmed, one would expect to see approximately equal proportions of comments related to hard and soft factors for the balanced firms and a bias towards comments related to hard factors for the qualityoriented firms. While the former is confirmed the latter is not. Quality-oriented firms discussed the mix of hard and soft factors in approximately the same proportions as did the balanced firms.

In conclusion, the evidence does not support Proposition 1. On the contrary, the evidence is that all of the case firms use a mixture of hard and soft factors of TQM. While there appear to be differences in the specific tools emphasized by balanced vs. quality-oriented firms (as shown in Table 12), these differences cannot be distinguished on the basis of the 'hard vs. soft' tool distinction often discussed in quality management literature $[21,48,49,64]$. Hence, the 'hard' vs. 'soft' factor distinction may be too simplistic for distinguishing between firms that emphasize primarily quality performance and those that emphasize both quality and innovation performance. 
Proposition 2 - Narrow vs. Wide Scope of TQM Implementation

Based on the definitions of the categories of firms, this proposition may be restated as:

- Proposition 2 (restated) - balanced firms emphasize practices in the three fundamental TQM principles of People Management, Process Management and Customer Focus while quality-oriented firms lack emphasis in at least one of these areas.

As with Proposition 1, the most straightforward approach to address this proposition is to examine Table 11 (revised with the summary results shown in Table 12) to look for indications of differences in the main categories of People Management, Process Management, and Customer Focus. In particular, an extreme example would be if balanced firms placed greater emphasis in all of the practices related to any of the three themes ${ }^{4}$. Conversely, the proposition would be disconfirmed in the event that 1) all of the practices were similar between the firms or 2) quality-oriented firms placed greater emphasis on some of the practices. In short, the more practices that receive greater emphasis in balanced firms, the more evidence there is to support the proposition.

The cross-case analysis provides evidence that balanced firms place greater emphasis in specific practices in all three of the areas. The evidence is strongest for Customer Focus - balanced firms emphasized two practices ('dissemination of customer needs' and 'proactively interacting with customers/ actively seeking customer input') to a greater extent than did quality-oriented firms. Also for Customer

\footnotetext{
${ }^{4}$ As with Proposition 1, the same limitation of assuming equal importance for the practices is acknowledged.
} 
Focus, one additional practice ('involving customers in product design') was identified as 'mixed', i.e., one of the balanced firms emphasized the practice more than the quality-oriented firms, whereas the other balanced firm did not. With regards to People Management, balanced firms emphasized one practice ('ensuring employee satisfaction') to a greater extent than quality-oriented firms and another practice ('organization-wide training \& development') was mixed. Lastly, for Process Management, the results are 'mixed' for two practices ('concept of the internal customer' and 'use of statistical techniques') Further, none of the firms placed much emphasis on two practices in this area ('standard operating procedures' and 'supplier rating system.'). Hence, the evidence is the weakest for differences related to Process Management. Taken together, there is evidence in support of the proposition.

Unlike with Proposition 1, simply tallying comments related to each of the themes (People Management, Process Management, and Customer Focus) may be misleading for this proposition. For example, knowing that a firm made ' 25 comments related to Process Management' whereas another made ' 16 comments' does not reveal the breadth or depth of the content of the comments. That is, the firm that made 16 comments may have been discussing a wide variety of Process Management practices in use by the firm whereas the other may have made all 25 comments about the same practice. Therefore, the cross-case analysis stands alone as the available evidence for this proposition. 
In conclusion, there is moderate evidence to support Proposition 2. In

particular, there is evidence that balanced firms have a wider scope of implementation of TQM, particularly with regards to practices related to Customer Focus, and to a lesser extent People Management. The evidence related to emphasis on Process Management practices is mixed and in fact, there is evidence to suggest none of the firms placed much evidence on several of the practices.

Proposition 3 - Internal vs. External Focus

As with the other propositions, rewording this proposition to reflect the terms used in the research helps to more effectively address the proposition. Further, given that the proposition contains several distinct facets, it is divided into three components to simplify analysis:

- Proposition 3a-Both quality-oriented and balanced firms emphasize People Management and Process Management (internal focus)

- Proposition $3 b$ - Quality-oriented firms' approach to customer interaction as well as to understanding competitors is primarily reactive or passive (internal focus); balanced firms take a more proactive approach to customer interaction and to their competitors (external focus).

- Proposition 3c-Quality-oriented firms view customers narrowly, i.e., 'direct' customers - the entities to whom they sell their products (internal focus); balanced firms view customers more broadly to include end users (whether or not they are direct customers), other stakeholders, their communities, and the environment (external, 'systems' focus).

Each 'sub-proposition' is examined separately followed by the overall conclusion related to the proposition. 
The results of the cross-case analysis again serve as the evidence to examine proposition 3a. Strong evidence in support of the proposition exists to the extent that the firms all place similar emphasis on the two themes listed - People Management and Process Management. Conversely, the proposition would be disconfirmed in the event that one category of firm placed greater emphasis on all the practices in People Management and/or Process Management.

The cross-case analysis suggests that both categories of firms emphasized People Management and Process Management, at least to some extent. While balanced firms placed greater emphasis on one practice in People Management ('employee satisfaction') the results are mixed for the other practice in this theme ('organization-wide training \& development') and for two practices in Process Management. And as stated previously, there were several practices in Process Management for which neither category of firm placed much emphasis.

As a result, there is moderate support for Proposition 3a indicating that both balanced and quality-oriented firms have an internal focus with respect to People Management and Process Management.

The cross-case analysis as reported above (and shown in Tables 11 and 12) is less helpful with respect to evaluating Proposition 3b. For this proposition, coding was conducted to 1) directly examine whether a firms' approach to customer interaction was reactive or proactive and 2) in general, how a firm talked about its 
competitors. For the latter point, comments about benchmarking were particularly relevant. For the former point, it was noted previously (and shown in Table 12) that 'proactive interaction with customers...' emerged as a slight revision to the results of the cross-case analysis on the Customer Focus practices of 'actively seeking customer inputs' and 'measuring customer satisfaction.' Additional evidence in support of this proposition includes statements from the balanced firms indicating these firms took the initiative to interact with their customers:

"our main customers, we try to get them out here once a year and let them tour the facility. No matter how many times customers come and visit, every time... a guy could be here four or five years in a row and say the 5th year he comes, 'I didn't know you had that!"”

Case firm $B_{1}$

"we really spend a lot of time making sure our website is updated, it's full, it's complete, so they can learn about any product and any process in the company. So we do... we use it a lot."

Case firm $\mathrm{B}_{1}$

"What that allowed us to do is basically... to be able to, if we saw sticks in the air type of thing we could stop by, measure it up..."

Case firm $B_{2}$ - 'sticks in the air' refers to the wall studs and trusses of a new home being built. Reference is with regards to proactively offering bids to customers.

"We contacted a couple, an architect and a couple design guys there in [city] and, hey we think this would be a good service for your customers."

Case firm $\mathrm{B}_{2}$

By contrast, in general, quality-oriented firms indicated more of a passive interaction with customers:

"You have to have a sound business model. And I think what that comes down to is matching the markets with your technological capacity."

Case firm $\mathrm{QO}_{1}$ - implication appears to be that the firm is focused on reacting to what customers (the markets) are demanding. 
“The R\&D comes in what the marketplace will take today that it didn't take a year or two years or three years or four years ago. Herein lies the difference and for us, that's the R\&D. Have we looked at a few new products? Sure."

Case firm $\mathrm{QO}_{1}$ - in particular, the 'what the marketplace will take' statement suggests a passive or reactive approach.

"Business is nearly entirely from word-of-mouth."

Case firm $\mathrm{QO}_{2}$

"they found us to be a little more responsive to call us say there's a problem and we'd get on it and take care of it."

Case firm $\mathrm{QO}_{2}$

With regards to benchmarking, this practice was addressed in the cross-case analysis and highlighted in Table 12 as a practice for which balanced firms placed greater emphasis than did quality-oriented firms. Therefore, the evidence supports Proposition $3 b$.

With respect to Proposition 3c, again, coding specific to this proposition was required. In particular, the coding examined 1) if firms discussed customers beyond the entities to whom they sold their products and 2) the extent to which companies discussed other stakeholders such as their communities and the environment. The fact that both categories of firms provided evidence of these activities indicates a lack of support for this proposition. Examples of statements from the websites and interviews include:

"... a dynamic company, that cares about its products, service, and community. [Company name] employees regularly participate in community events, and raise money for charities... [patented process] is efficient, uses clean electricity, and releases zero negative emissions."

Case firm $\mathrm{B}_{1}$ (from company website) 
"We thought it [proactive bid process] was good for the design guy because... And we thought it was good for the homeowners because... And we thought it was good for the contractors because... And we thought it was even good for our competitors because they wouldn't have to do design."

Case firm $\mathrm{B}_{2}-$ Broad focus on customers; justifications for the first three stakeholders are not shown to save space.

"Maybe a better example I can give you is SFI vs. FSC certification. We're both." Case firm $\mathrm{QO}_{1}$ - both these certifications are intended to ensure consumers that products were sourced sustainably from 'well-managed forests' - evidence of concern for the environment.

"The homeowner had made a comment when he dropped these off about it in his mind the beams were going to be the same as his walls on the house. So I said, no he's showing 10-foot plates and now they're going to be nine-foot plates. And I said you want your beams even with that nine-foot plate, and he said yeah. So I said, OK, I'll design it that way. Even though my design isn't going to match the plan it's what the homeowner wants."

Case firm $\mathrm{QO}_{2}$ - reference to focus on end 'user' (homeowner) vs. direct customer (contractor/builder)

In conclusion, there is evidence in support of two of the three sub-elements of

Proposition 3. Specifically, evidence indicates that both balanced and quality-oriented firms have an 'internal' focus as reflected by emphases on People Management and Process Management. Also, there is evidence that balanced firms take a more proactive approach to customers and competitors (benchmarking). However, the evidence suggests that both categories of firms see their firm in the broader systems context that includes direct and final customers, as well as their communities and the environment.

While the preceding propositions were those that were developed based on the review of the literature and prior to data collection, one additional proposition emerged during the data collection and analysis. As the interviews were being 
conducted, during interview transcription, and during coding, apparent differences in company culture emerged. While company culture was not explicitly examined in the research (aside from passing reference in a question on Leadership), it was difficult not to notice the differences while talking with interviewees at their place of work. The proposition may be stated as:

Proposition 4: Companies that are more proactive/strategic will achieve greater performance with respect to quality and innovation than firms that are more reactive/conservative.

Again, the proposition may be reworded to fit the terms used in the research:

- Proposition 4 (restated): Balanced firms will be more proactive/strategic while quality-oriented firms will be more reactive/conservative.

This proposition is deliberately vague with respect to the specific practices or areas (e.g., strategic planning, customer interaction, supplier relations, etc.) where a firm may be considered either proactive or reactive. The evidence will show that the differences exist in several areas and as such appear to be more overarching, i.e., 'cultural.'

Several of the quotes presented above provide evidence of the 'proactive vs. reactive' distinction. For example, for Proposition $3 \mathrm{~b}$ above, the evidence suggested that the balanced firms proactively interacted with customers whereas quality-oriented firms were more reactive in this regards. Other evidence in support of the proactive/strategic emphasis for balanced firms includes: 
"like I came across the [ultrasound technology]. And I did a lot of research on my own, just saying 'hey could we ever apply this to [our product line]?'... out of the 100 ideas that I bounce off people in the company, 90 of them don't go anywhere. Ten of them go far enough that I'll actually do something. And maybe only one or two will stick."

Case firm $B_{1}$ - exploration of new technology; willingness to experiment and take risk "we try a lot of... different things around here... There's nothing we won't consider." Case firm $\mathrm{B}_{1}-$ culture of experimentation

"one of the challenges we have is when you get a twisted beam... What we've done is we've found a manufacturer in Ontario Canada who had this particular horizontal saw that they used for logs... So we were able to use the [trade name] and we got them to adapt it for our beams."

Case firm $\mathrm{B}_{1}$ - working with equipment vendors to develop process innovations

"So I wanted to get a good showing on this commercial... so that's when we bought a new edgebander for that project. We saw houses were starting to slow down some. Well, if we get into this commercial..."

Case firm $\mathrm{B}_{2}-$ shift in business strategy to enter new market segment

"We talked to the two different vendors over in Seattle, hitting the whole area up there. And no one was doing a type two PVA glue..."

Case firm $\mathrm{B}_{2}$ - efforts to identify new suppliers for new product line

"So he [employee] spent the next year, probably 60 to 80 and even up to 90 hours a week developing the software program. And it worked pretty good. We still... to try and get it out on the market and sell the program we'd have to create a whole Help section but...So anyhow we developed the whole software too for doing this counter program."

Case firm $\mathrm{B}_{2}$ - efforts to develop software for product innovation

Evidence in support of the more reactive and conservative culture in quality-

oriented firms includes:

"when we were busy we were just buried, I mean bring in a complicated job we ain't got time to mess with it - just take it somewhere else... of course when things crashed now we're like OK, time to learn how to go out and sell."

Case firm $\mathrm{QO}_{2}$ - reactive approach to changes in the market

"...we've never really done a true assessment of what it actually costs us to do this and do that. They say there's a lot of tools in our software that we could be utilizing." Case firm $\mathrm{QO}_{2}$ - passive or conservative approach to process management 
"[How do you conduct R\&D?] We really don't. Outside of, like I say if there's stuff other companies are providing that our customers are asking about." Case firm $\mathrm{QO}_{2}-$ Reactive business strategy

In short, the initial propositions and the emergent proposition suggest the following general distinctions between the categories of firms:

- Balanced: These firms are proactive and strategic. They are amenable to risk and experimentation. As noted previously, the firms appear to view innovation as a means to achieve some other goal such as product quality, improved process efficiency, etc.

- Quality-oriented: These firms are reactive and conservative. They are riskaverse and focused on meeting present needs or 'catching up' to the competition. Innovation is viewed as an expense or simply as 'technology'; and "technology is wonderful when it's proven."

\section{Enfolding Literature}

As stated by Eisenhardt [86], this phase of the research is particularly concerned with testing the reliability and external validity of the findings. Greater generalizability is attained to the extent that findings concur with existing literature.

Given the focus of the research on relations between quality management and innovation, particular emphasis is given to the existing literature related to this topic. This literature was discussed in the review of the literature and summarized in Table 6. The approach here is to restate the key findings from each study described in Table 6 (as well as several others that are relevant to the topic of quality management as it relates to innovation) and to then compare and contrast the findings presented in the current research. 
The section concludes with a discussion of the context of the forest industry and how this industry's structure and current context may influence the findings. Attention is given as well to findings in the literature related to innovation in the forest industry that either serve to confirm or disconfirm the present findings.

\section{Literature Related to Relationships between Quality and Innovation}

Sitkin et al. were among the earliest authors to discuss possible linkages between quality management and innovation [24]. While the focus of their study is not strictly on the relationship to innovation, the connection between innovation and learning is alluded to by stating that firms must balance stability and reliability (i.e., control) with exploration and innovation (i.e., learning). These authors presented a contingency approach to implementing TQM by proposing that Total Quality Control (TQC) firms emphasize satisfying existing customers and exploiting existing skills and Total Quality Learning (TQL) firms emphasize scanning for new customers and exploring new skills and resources. Findings here confirm these authors' assertions in that both quality-oriented firms (which may be analogous to TQC firms) emphasized satisfying existing customers and only expressed interest in identifying new customers or markets to the extent that "when things crashed" (quoting case firm $\mathrm{QO}_{2}$ ) the firm then recognized it needed to find new customers. Similarly, firm $\mathrm{QO}_{1}$ talked about new product development in the context of 'matching the market' to their existing production capacity and distribution system. 
However, Sitkin et al. also stated that 'benchmarking' was a practice emphasized by TQC firms to the extent that it is geared to better understanding existing customer needs. Findings of the present research disagree with these findings in that the TQL (balanced) firms emphasized benchmarking whereas the TQC (quality-oriented) firms did not. This disagreement may well be in how the authors define the term and the specific focus of benchmarking. Rather than being focused on identifying industry best practices (as done by TQL/balanced firms), the authors are referring to identification of customer needs.

In discussing "Overcoming the TQM barrier to innovation", Samaha states that "Quality is doing things better; innovation is doing things differently" [31]. In particular, the author sees benchmarking as 'perilous' with respect to innovation in that benchmarking is a means to 'catch up' to the competition. Again this assertion disagrees with the present research in that both balanced firms emphasized benchmarking. The distinction may be in the specific focus of the benchmarking efforts - benchmarking firms within the industry may lead to this 'catch up' mentality, whereas benchmarking best-in-class outside one's industry may lead to greater innovation performance. In that the case firms were not asked explicit questions about whom they benchmark, it is not possible to hypothesize further.

Based on an extensive review of the quality and innovation literature, Prajogo and Sohal proposed several areas where TQM may have either a positive or negative impact on innovation performance [30]. Several of these arguments are presented 
below followed by a brief discussion of how the present research either agrees or disagrees. With regards to customer focus, the authors state:

- Positive - TQM will encourage organizations to be innovative because they have to seek a better way to meet and exceed customers' requirements.

o This statement is confirmed to the extent that the balanced case firms proactively sought to interact with their customers.

- Negative - TQM could lead organizations to be reactive in responding to customers' needs; may prevent organizations from exploring unserved needs and markets; and may prevent organizations from developing radical new products because of its inherent risk-avoidance philosophy.

0 All three of these assertions are confirmed by the quality-oriented case firms; evidence for this was presented above in the section on Shaping Propositions.

With respect to continuous improvement, Prajogo and Sohal state:

- Positive - TQM will encourage change, innovation, and creative thinking in how work is organized and conducted.

o This statement is confirmed with regards to the Leadership practice emphasized by balanced firms - "Senior managers actively encourage change and implement a culture of improvement, learning, and innovation..."

- Negative - the establishment of a regulatory standard could inhibit innovation because it reduces the ambiguity of a task that is necessitated to enforce innovation.

o Findings of the present research are 'mixed' on this point. In contradiction to this statement, in spite of a regulatory standard, one balanced firm sought to differentiate itself from competitors by developing a process innovation that would enable the firm to exceed the standard. And in concurrence with the statement, one qualityoriented firm stated that their quality efforts were simply geared to meeting the standard.

Lastly, with respect to continuous improvement, Prajogo and Sohal state:

- Positive - employee empowerment should make people feel they have a certain degree of autonomy, are less constrained by technical or rule-bound aspects, and self-efficacious in doing their work, which will make them innovative.

o This was confirmed by firm $\mathrm{B}_{1}$ by the comment, "We rely on our supervisors to tweak what's there..." Further, there was little evidence 
that the quality-oriented firms empowered their employees thus demonstrating that lack of empowerment may be correlated with lack of innovation performance.

- Negative - while conceptually empowerment and involvement are very much congruent with innovation, in practice, workers are usually "empowered and involved" to deal only with execution and small scale of improvement.

o This statement is disconfirmed by the present research in that case firm $\mathrm{B}_{1}$ limited empowerment to a worker's immediate sphere of influence. Regardless, the firm found that workers' ideas for improvement were not limited to 'small scale improvement.'

In short, Prajogo and Sohal's pro and con arguments for how TQM may impact innovation are mostly confirmed by the case firms. The fact that it is possible that both of their pro and con arguments for how TQM may impact innovation could be confirmed is testament to the fact that specifically how a company implements TQM can significantly influence the performance outcomes with respect to quality and/or innovation.

While the literature discussed above emphasized hypothesized relationships between quality management and innovation, several authors have also conducted research to test these relationships. For example, Lin and Lu explored a causal relationship between quality and innovation in examining product innovation in the automotive industry [80]. Results showed that "automakers actively engage in product innovation if they produce high-quality products", or more simply that "product quality determines product innovation." Both balanced case firms confirm this finding in that the firms developed product innovations as a means to improve product quality. For example, case firm $\mathrm{B}_{1}$ developed a new product line by 
guaranteeing certain aspects of product grade ${ }^{5}$ and similarly, case firm $\mathrm{B}_{2}$ developed a new countertop with a 10-year warranty (durability dimension of quality).

Bossink conducted a case study to examine the use of specific quality tools for an innovative homebuilding project in the Netherlands for which results suggest a supportive function of quality in the management of innovation [100]. Further, it is suggested that quality tools are used implicitly and sometimes explicitly to manage innovation, for example, benchmarking and customer satisfaction measurement are used in both quality as well as innovation management; a key finding was that customer satisfaction measurement contributed to the initiation of innovation. The finding with respect to benchmarking is confirmed in that benchmarking was identified as a practice emphasized by both balanced case firms but not by the qualityoriented firms. However, as stated previously, while none of the case firms explicitly measured customer satisfaction, the balanced firms took a more proactive approach to interacting with customers. And given that this interaction was often discussed in the context of discussions about customer feedback, perhaps 'proactive interaction with customers' may be conceived as a 'low-intensity form' of customer satisfaction measurement. And by contrast, one may speculate that 'reactive customer interaction' does not have the same impact on innovation to the extent that the relationship is driven by the customer rather than the firm. This may be an area where further research is warranted.

\footnotetext{
${ }^{5}$ Unfortunately, it is difficult to be more explicit here about the features without risking violation of confidentiality assured to respondents/interviewees.
} 
McAdam et al. examined the organizational progression from quality to innovation in 15 small (fewer than 100 employees) firms in Ireland [23]. The firms were evaluated based on quality and innovation award criteria and given a score for innovation and continuous improvement. The authors report that there were significant similarities between firms that got high scores on both quality and innovation. The authors state that Total Quality “...lays the foundation for a cultural environment that encourages innovation." It is difficult to determine if this finding is confirmed by the present research in that cause and effect are not clear. While there were apparent cultural differences between the balanced and quality-oriented case firms, it is not clear if Total Quality 'laid the foundation' for the culture that encouraged innovation in the firms or if the culture influenced how the firms implement quality management.

Singh and Smith explored “... whether TQM can be used as a vehicle for inculcating innovation" in Australian manufacturing firms [33]. The authors concluded there was insufficient evidence to link TQM and innovation and suggested "It could well be that the TQM constructs and innovation are related in a more complex way." Further, an unexpected result of the test for construct validity in the research was that the construct Product/ Process Management led to poor fit of the model; use of SPC in particular was eliminated from the model. These authors' findings are confirmed by the present research in that all four case firms used the TQM constructs with varying levels of emphasis, however the mix of practices and emphases that differ between the firms attaining innovation performance and those 
that do not is quite complex. See for example the discussion of Proposition 1 above related to the use of hard and soft factors of TQM. Further, the authors' findings with regards to Process Management are confirmed as well in that specific practices in this area received little emphasis by the case firms and the practices that did appear to differ were 'mixed', i.e., one balanced firm emphasized the practice whereas the other did not.

In addition to proposing pro and con arguments for how TQM might impact innovation, Prajogo and Sohal took the additional step of conducting research to study the nature of the relationship; firms studied include manufacturing and nonmanufacturing firms in Australia. In one of their first papers [25], results showed that TQM significantly and positively relates to quality performance as well as innovation performance. Further, they found significant correlations between product quality, product innovation, and process innovation. Similar to Lin and Lu's findings in the automotive industry, Prajogo and Sohal suggested the possibility of 'crossfertilization' between quality and innovation performance. Specifically, they found that process innovation may mediate the relationship between product quality and product innovation. The findings of the present research confirm this concept of 'cross-fertilization' in that, as stated previously, the balanced case firms pursued innovation as a means to achieve quality. For example, case firm $\mathrm{B}_{2}$ developed a product innovation to improve quality (durability); further, this product innovation required the firm to develop process innovations in order to be able to manufacture the new product. 
In another study, Prajogo and Sohal conducted a case study in an Australian automotive components manufacturer to examine the transition from TQM to 'Total Innovation Management' [82]. One key finding was that the transition in the case study firm resulted in customer relationship management shifting from reactive (responding to customer complaints) to being proactive. Further, the firm shifted its focus from direct customers to end users. While the finding of proactive customer relationship management is confirmed by the present research, the latter finding related to focus on end users is not. Case firm $\mathrm{QO}_{2}$ demonstrated significant investment in working with end users (homeowners in their case) even though the direct customers were contractors/homebuilders. A final conclusion of interest was reinforcement of the 'chain reaction' (what these authors termed 'cross-fertilization' in a previous article) between process innovation and product innovation; the case firm implemented automation (process innovation) to improve product quality and was then 'forced' to innovate new products that were suited to the technology. Again, this finding is confirmed by both balanced case firms in the present research - the firms pursued both product and process innovation as a means to improve quality performance.

And finally, with respect to research conducted by Prajogo and Sohal, these authors extended their research to study the impact of integration of TQM and the concept they described as Total Innovation Management (TIM) on performance in Australian manufacturing and service firms [35]. TIM is defined to include technology management and R\&D management. The authors highlight the 
importance of organizations developing capacities to simultaneously balance

exploitation (maximizing existing resources for efficiency and productivity) and exploration (maximization of capabilities to develop new skills and resources). The results contrasted with these authors' prior research on relationships with innovation TQM showed no significant relationship with innovation performance. However, there was a strong and positive correlation between TQM and TIM. Significant relations were also shown to exist between product quality and process innovation and between product innovation and process innovation. The authors state again that these findings suggest 'cross-fertilization' between these performance variables. Given the strong correlations between TQM and TIM, the authors proposed integrating TQM and TIM principles and offered two specific areas where this might be achieved:

- Customer focus - organizations should strive to balance efforts to meet current customer needs with R\&D focused on meeting unserved needs. Similarly, organizations should serve customer needs through product innovation that is technology push rather than market pull.

o The present research confirms these findings and the author's choice of verb 'balance' is particularly germane here. Both balanced case firms exhibited such efforts to proactively serve existing customers while at the same time, developing new products to exceed customer needs and attract new customers. Also, their technologies were 'pushed' in that both case firms described how they worked to educate customers (existing and new) about the benefits of their new products.

- Process management - organizations should strive to balance control and continuous improvement with technology management that is more focused on radical innovation.

o Unfortunately, the authors provide no further details regarding this point. Therefore, it is difficult to evaluate how findings of the present research may agree or disagree. Case firm $\mathrm{B}_{1}$ 's differing approaches to process management and product innovation may serve to confirm the point. Specifically, the interviewee described their approach to process management as quite rigorous whereas product innovation that, in the interviewee's words was more "loosey-goosey", indicating that at a 
minimum, the firm recognized the need to adapt the approach to the goal.

Lastly, with regards to the literature exploring relationships between TQM and quality and/or innovation, Hoang et al. also tested these relationships; however, these authors took the additional step of attempting to address the impacts of specific TQM practices on innovation [104]. The authors studied manufacturing and service firms in Vietnam. Study results showed that TQM has a strong and positive relationship with two measures of innovation - 1) level of newness and 2) number of new products and services developed. However, as there were significant problems with multicollinearity within the TQM constructs, the authors developed composite measures for several of the constructs including: Leadership \& People Management (including the constructs for Top Management Commitment, Employee Involvement, and Employee Empowerment) and Process \& Strategic Management (including the constructs for Process Management, Information \& Analysis, and Strategic Planning). Using the composite measures, results showed that Leadership \& People Management was significantly related to level of newness; only Education \& Training and Process \& Strategic Management had significant relationships with both measures of innovation. The authors concluded that not all TQM factors have an impact on innovation performance.

Findings of the present research confirm Hoang et al.'s findings in several ways. At a minimum, their finding that "not all TQM factors have an impact on 
innovation performance" validates the main thesis of the present research in attempting to identify those specific practices that do impact innovation performance. And with respect to the positive impact of Leadership \& People Management, the present research identified specific practices within these areas receiving greater emphasis by balanced case firms vs. quality-oriented firms. However, Hoang et al.'s findings with regards to the positive impacts on innovation of Education \& Training and Process \& Strategic Management are generally not confirmed by the present research. Results of the cross-case analysis were mixed with regards to 'organizationwide training \& development.' Further, while the practice of 'structured planning, long and short-term goals' was one for which there were apparent differences between balanced and quality-oriented firms, in general, the practices related to Process Management and Strategic Planning received little emphasis by any of the case firms.

Forest Industry - Structure, Current Context, and Innovation

The preceding section addressed 'enfolding the literature' from the viewpoint of relationships between quality and innovation without consideration of industry sector. The author is not aware of any research on this topic specific to the forest industry however the topic of innovation in the forest industry is receiving increasing attention from the research community in recent years. In fact, several university centers and research institutes have been created since 2005 with a focus on innovation in the forest industry. Examples in North America alone include:

- the Oregon Wood Innovation Center at Oregon State University, created in 2005 (for which the author serves as Director); 
- change in name, and research focus, of a Canadian forest industry research institute from Forintek Canada to FP Innovations in 2007;

- creation of the Center for Wood Innovation \& Sustainability at the Pennsylvania State University in 2009; and most recently,

- formation of the Wood Innovation Research Group at Virginia Polytechnic Institute \& State University in 2010.

Therefore, a more thorough examination of the findings of the present research must include some discussion of the structure and context of the forest industry and research related to innovation in the forest industry as well.

The forest industry includes a wide variety of firms that either use wood as their primary raw material or firms that provide manufacturing equipment to this industry. The general nature of all of these firms is that the markets for finished products are very closely linked to new home construction and/or remodeling. Case firms here were no exception; all four firms produced products for new home construction and remodeling; products included:

- $\mathrm{B}_{1}$ - large beams and timbers for high-end homes

- $\mathrm{B}_{2}$ - custom kitchen and bath cabinets

- $\mathrm{QO}_{1}$ - remanufactured products such as door and window trim, interior paneling and exterior siding

- $\mathrm{QO}_{2}$ - roof and floor trusses

Like most industry sectors, significant restructuring has occurred in the forest industry as a result of globalization. For example, prior to the early 1990s, much of the pine lumber used by U.S. wood window and door manufacturers came from ponderosa pine forests in the western U.S. [111]. Beginning in the mid-1990s, an 
increasing share of this lumber now comes from radiata pine plantations in the southern hemisphere, with New Zealand and Chile being the leading exporters [111].

As another example, the eastern U.S. was at one time a leader in furniture production. Hardwood lumber (e.g., oak, maple, walnut, cherry, etc.) for furniture was sourced from hardwood sawmills located in the eastern U.S. Today the majority of furniture sold in the U.S. is imported, and a large share comes from China and other Asian nations. For example, in 1992, 19 percent of furniture sold in the U.S. was imported; by 2008 that value had risen to 64 percent [112]. Further, the dollar value of Chinese furniture imports in the U.S. increased from $\$ 4.9$ billion in 2000 to $\$ 14.0$ billion in 2008; in the same time period, imports from Vietnam increased from $\$ 13.3$ million to $\$ 1.4$ billion [113]. Raw materials (lumber, plywood, etc.) for the furniture come from eastern U.S. hardwood sawmills as well as from other mills all around the globe [112].

As previously discussed, the case firms for the present research were small firms. None were 'global players' in the market with respect to product sales; all four case firms sold either locally or within North America. However two of the firms ( $\mathrm{B}_{2}$ and $\mathrm{QO}_{2}$ ) discussed sourcing their raw materials from global sources, e.g., hardwood plywood from China and pine lumber from Chile, respectively.

Of particular relevance to the present research is the impact of the ongoing global recession at the time the data were being collected. Industry analysts have 
stated that this was one of the worst recessions to impact the forest industry in decades [114]. Housing starts are often used as an indicator of demand for forest products. According to the U.S. Census Bureau, housing starts declined from a peak of 1.4 million in 2004 to a record low of 445,000 in 2009 [115]. Reports were widespread in 2008 and 2009 of impacts the decline in the housing market was having on the forest industry. For example, in April of 2009, Portland, Oregon-based Hampton Affiliates announced:

"Continuation of the global financial recession, the severe housing market decline, and the resulting impact on Hampton's businesses make it prudent for us to make these adjustments in our operating plans. Customer demand and lumber prices are at historically low levels and most forecasts predict it may be several years before normal housing markets return. Hampton's sawmills at Tillamook, Oregon, and Darrington, Washington, will drop to $60 \%$ of normal operating hours..." [116]

The segment of the forest industry that was the target population for this research was clearly impacted by the recession. While it is unknown how many of the surveys (298 out of 3305) were returned as undeliverable due to companies going out of business, in the efficient set (following cluster analysis) at least one of the three candidate balanced case firms had shifted its operations from manufacturing to wholesale between when they responded to the questionnaire and when they were contacted to request an interview. Thus, at the least, the recession likely impacted response rate as firms were struggling to remain in operation as well as the direct reduction in the pool of candidates for case studies. 
Further, all four case firms discussed the impacts the recession was having on their businesses. In fact, while no explicit attempts were made to examine case firms' performance with respect to profitability, all of the firms made statements related to their success in the present economy. For example:

“As residential slowed down and we've got this \$200,000 cabinet job and counter job, we think that will get our foot in the door to bigger commercial and so that's why we were willing, even though the slow down, to go ahead and get this edgebander." Case firm $\mathrm{B}_{2}$ - investment in new technology/equipment for new market

"I think our actual technology is probably behind our competitors. You know from the standpoint of degree of automation it probably is. The difference is that we're making money while our competitors aren't."

Case firm $\mathrm{QO}_{1}$ - justification for not investing in technology

Therefore, in some way, the recession may have served as a 'proxy' of sorts for profitability and validation of the efficacy each case firm's business strategy in that the firms were still operating at the time of the research whereas so many of their competitors had either curtailed operations (e.g., gone from three shifts to one) or gone out of business altogether. These comments also further serve to validate the categorization of the case firms - the balanced firms provided evidence of their strategy to innovate (or at least adopt innovations) to remain competitive whereas the quality-oriented firms discussed the fact that not investing in innovation (primarily process innovations) was a key factor in their success.

With the preceding as a brief overview of the forest industry and how the context at the time of the research may have impacted findings, attention is now turned 
to the literature exploring innovation in the forest industry. In particular, literature is examined with respect to how past findings relate to that of the present research.

As discussed previously, research has revealed little innovation, particularly product innovation, in forest products manufacturing. Hovgaard and Hansen state that, despite the fact that innovation has "long been accepted in the business literature as instrumental to company success, very little research has been done specific to the forest products industry" [52]. These authors conducted qualitative research to examine small forest products firms (one to 60 employees) in Alaska and Oregon to examine how these firms viewed innovation. Although the authors examined innovation in three categories (product, process, and business systems), the most common concept of innovation by study respondents was "having a unique product or process." The results of the present research confirm this finding to the extent that product and process innovation distinguished the balanced case firms from the qualityoriented firms whereas performance related to business systems innovation was similar between the two categories of firms.

Further, Hovgaard and Hansen identified six steps in the product development process practiced by respondents; however, respondents did not generally undertake consistent, structured processes for product development. This finding is also confirmed by the present research in that the balanced case firms, although involved in new product development (NPD), could not describe a structured process. Further, 
one case firm explicitly described the NPD process as "loosey-goosey" in contrast to the rigorous approach the firm used with respect to process innovation.

Crespell et al. examined innovativeness in the sawmilling sector of the North American forest industry [117]. The authors state that the forest industry has traditionally maintained a focus on a low-cost strategy concentrating on wood fiber recovery (i.e., yield). The present research confirms this statement in that all of the firms discussed the importance of fiber recovery. For example,

"...the software was working great, the CNC the nested base, minimal waste, maximum yield."

Case firm $\mathrm{B}_{2}$ - discussing computer numerically controlled (CNC) equipment using optimization techniques ('nesting') to maximize yield of cabinet parts from each panel (plywood or fiberboard).

"The mainstream products are not what you make money at; you make money on what you do with your downfall."

Case firm $\mathrm{QO}_{1}$ - 'downfall' is waste; implication is that firms must maximize yield by finding markets for, or producing products from, the waste.

These comments further solidify the distinction between balanced and qualityoriented case firms; while both firms focus on yield ('fiber recovery'), balanced firms do so via processing innovations to minimize waste and maximize yield, qualityoriented firms emphasize yield via finding markets for waste.

Crespell et al. surveyed sawmills regarding their practices related to innovativeness and found that respondents rated themselves more innovative with respect to manufacturing processes as compared to product and business systems 
innovations. These findings are also confirmed in the present research with respect to the balanced case firms focus on process innovations and the lack of business systems innovation as being a distinguishing factor. However, in contrast to these authors' findings, balanced case firms emphasized product innovation as well. This latter finding is likely influenced by the fact that Crespell et al. focused on sawmills whereas the present research focused on the forest industry more broadly (only one of which would be characterized as a sawmill, and certainly not a 'traditional' sawmill).

Peters et al. investigated characteristics of recent technology innovations in particleboard and composite materials [102]. The authors identified four major clusters of technology innovations - manufacturing, high technology, materials processing, and new products; equipment makers dominated innovation in all of these clusters. Further, "improved product quality" was the predominant source of economic benefits identified. Findings from the present research confirm these findings as well, in that balanced case firms discussed either purchasing (i.e., adopting) process innovations and/or working directly with equipment producers to refine the equipment to suit their specific needs. Further, the finding of "improved product quality" as the principal economic benefit is particularly relevant to the present research and further supported by the general literature on quality and innovation with respect to 'cross-fertilization' between innovation and product quality $[25,80]$. That is, innovation (both product and process) is often the means by which higher product quality is achieved, as was the case in both balanced case firms. 
Lastly, in a study of the Spanish wood products industry, Diaz-Balteiro et al. examined correlations between firm efficiency and innovation. The authors found little correlation between efficiency and innovation. Further, they report that there is a “...low firm priority towards R\&D as a means to achieve competitiveness and an innovation strategy followed by many Spanish firms based on the acquisition of embodied technology available in international markets" [81]. These findings may be confirmed to the extent that the balanced case firms are unique in their emphasis on innovation and therefore valid case candidates. Further the statement about 'embodied technology', referring to innovation via adopting/acquiring processing technology, is confirmed by the present research and by that of Peters et al. above in that firms in the forest industry rely on equipment vendors; balanced case firms here were no exception to this principle. However, the balanced case firms also worked closely with these vendors to adapt the equipment to their needs.

Conclusions that may be drawn from the 'enfolding the literature' phase of the analysis are that there is significant support in the literature for many of the findings of the present research. Such support is found in literature related to the forest industry in North America and other nations as well. Further, the findings are supported by existing literature related to other manufacturing sectors in nations around the globe. Hence, there is significant support for the generalizability of many of the findings beyond the context of the West Coast U.S. forest industry.

In particular, there is support in existing literature for the findings related to: 
- Proactive interaction with customers/actively seeking customer input;

- Encouraging change and implementing a culture of improvement;

- Giving employees opportunities to share in change;

- Viewing innovation as a 'means to an end'; and

- Benchmarking

Details on each of these points are addressed in the concluding section on Reaching Closure.

Findings in a few prior studies served to confirm the need for the present research. In particular, other researchers have discovered difficulties in quantifying the quality management-innovation relationship due to what are presumed to be complex relationships between the two. Hence, more in-depth comparison of specific practices as can be done via case research is needed to delve deeper into these relationships. One example of such a finding in the present research is related to Proposition 1 regarding the hard and soft factor distinction. As stated in the analysis of the proposition, such a distinction is likely too simple to capture the complex relationships between TQM practices and quality and innovation performance.

Prior research confirms the findings of this research on either the low emphasis placed on process management or simply the need for firms to balance their approach. Case firm $B_{1}$ 's rigorous approach to process management/process innovation contrasted with the firm's much less structured ("loosey-goosey") approach to product innovation may serve as a good case-in-point. At the same time, this suggestion 
contradicts recommendations by forest industry experts regarding the industry's need to use a structured approach to NPD.

Examination of the case firms in light of the forest industry structure and current context shows good correspondence - the case firms' markets are similar to those of the broad forest industry (home construction and remodeling), the firms are 'global' to the extent that two of the firms import raw materials from suppliers outside North America, and all firms were impacted by the recession. However on the latter point, the case firms' responses to the recession differed significantly. In fact, their responses serve to further validate the balanced/quality-oriented distinction and hence categorization of the firms. Balanced firms emphasized technology as one means to pursue new market opportunities beyond those most severely impacted by the recession whereas quality-oriented firms reported that their long-term strategy of avoiding investment in technology is what had allowed them to continue to be profitable.

\section{Reaching Closure}

As discussed in the chapter on Research Methods, this final stage of the research focuses on 1) when to stop iterating between data collection and theory development with the stop point generally being when it is determined that theoretical 'saturation' has been reached and 2) reporting results. The term 'saturation' indicates the point at which incremental improvement to the theory is minimal and therefore there is little point in collecting additional data. However, a modified approach was 
used here given that the 'stop point' was predetermined; the number of case firms (four) and interviewees per firm (one) were determined in advance. Therefore, 'saturation' was only achieved to the extent that additional cycles of within- and crosscase analysis revealed minimal additional insights.

With regards to reporting results, Yin recommends presenting results as responses to a series of open-ended questions [88]. Such an approach is well-suited to this research in that the title of this research can easily be stated in the form of an open-ended question:

What are the best practices in quality management for achieving quality and innovation performance in the forest products industry?

However, this question is perhaps too open-ended to be addressed in a practical manner. Addressing each of the research propositions (reworded to be stated as openended questions) is the approach taken here. Each question along with the answer is presented below.

1. What mix of 'hard' and 'soft' TQM factors should a firm use to achieve both quality and innovation performance?

While it appeared from the literature that this might be a reasonable approach to address the question, results from the research showed that this is not a useful distinction for determination of best practices. It is perhaps, overly simplistic for the complex relations being examined here. Case firms used a mix of all of the hard and soft factors. Therefore it is not whether a practice is analytical (hard) or human-centered (soft) that is important, but rather the 
specific nature of the practices themselves. Hence, we must look beyond this distinction.

2. How widespread do TQM practices need to be implemented within a firm in order to achieve both quality and innovation performance?

Note: this proposition was originally worded as 'wide vs. narrow' scope of implementation. This question was addressed not by looking at specific functional groups within a firm (in part due to the fact that the case firms were quite small) but rather from the perspective of fundamental principles of TQM - People Management, Process Management, and Customer Focus. It is assumed that practices within each fundamental principle will involve different areas and individuals within the firm, for example Human Resources for People Management, operations personnel for Process Management, and Sales \& Marketing for Customer Focus. In general, results showed that practices related to Customer Focus, and to a lesser extent People Management, were emphasized most by balanced firms. The evidence related to emphasis on Process Management practices is mixed and in fact, there is evidence to suggest none of the firms placed much emphasis on several of the practices. Of course, this is not to say firms should neglect Process Management, but rather that the areas of emphasis in seeking to adapt management practices should be those related to Customer Focus and People Management. Which specific practices should be emphasized and how is addressed in the succeeding propositions. 
3. What balance should firms seek with regards to "internal vs. external" focus with respect to:

a. People Management and Process Management (internal focus)

b. Interaction with customers (reactive $=$ internal focus, proactive $=$ external focus) and competitors (external)

c. How they view customers (direct customers = internal, final users and other stakeholders $=$ external)

As stated above, it is important that firms continue to maintain a focus on all three fundamental principles of TQM - including practices related to People Management and Process Management. While there was little evidence in the research for changes firms might make with respect to Process Management, one practice related to People Management that is worthy of emphasis is ensuring employees have opportunities to share in change and are encouraged to help the organization to implement change. This research as well as prior studies pointed to the importance of the TQM practice of employee empowerment and involvement with regards to innovation performance. And the present research indicated that this practice can even be effective in cases where empowerment and involvement are limited to workers' immediate sphere of influence.

Perhaps the most widely supported finding from the research is related to how firms interact with their customers. In this regard, the recommendation is to ensure that interaction with customers is proactive. Firms must strive to balance satisfying existing customers with seeking to identify new customers and explore unserved needs. Several authors have characterized this approach 
as balancing exploitation and exploration, where exploitation focuses on maximizing existing resources for efficiency and productivity (a key focus for the forest industry) and exploration focuses on maximizing capabilities to develop new skills and resources. While much of the research suggests that customer satisfaction measurement is a key factor, the present research indicates that simply taking a proactive rather than reactive or passive approach to customer interaction distinguishes balanced from quality-oriented firms.

Practices used by the two balanced case firms can be used to provide additional detail on 'proactive customer interaction.' These practices are discussed below in the conclusion to this section.

In addition, while some authors theorized that benchmarking may be 'perilous' to innovation due to inculcating a culture of playing 'catch up' to competitors, the key point here may be in how one defines benchmarking and in what the specific targets are for benchmarking. As defined here, the recommendation is to engage in an active competitive benchmarking program to measure performance against the 'best practice' in the industry.

With regards to how firms define their customers (as direct customers or more broadly), the evidence suggests that, as with the hard vs. soft factor distinction, this practice is not critical. 
4. What aspects of company culture may influence quality and innovation performance?

An emergent theme related to differences between balanced and quality-oriented case firms was related to organizational culture. Further the Leadership practice requiring that "senior managers actively encourage change and implement a culture of improvement, learning, and innovation in pursuit of 'excellence'" emerged as a practice emphasized more by balanced than quality-oriented firms. Many studies have pointed to the importance of top management commitment and the relationship to organizational culture. However, cause-and-effect is unclear here. Does culture influence how firms implement TQM? Or does the implementation of TQM positively influence the culture towards innovation performance? In general, the culture of balanced firms can be summarized as proactive and strategic; these firms are amenable to risk and experimentation.

Further, at the most fundamental level, how a firm views innovation may be a key factor related to organizational culture. Several authors discussed the concept of 'cross-fertilization' between quality and innovation. In particular, this concept is manifested in how firms may pursue product quality via product innovation. Further, process innovation may mediate the relationship by, for example, a process innovation may be required to enable a product innovation. Therefore, if senior managers in a firm view innovation as a 'means to an end', they may be likely to manage in such a way, even 
redirecting the company's strategy, such that the organization's culture reflects the shift.

Interview data and website information enable identification of more detail on specific areas of focus related to 'proactive customer interaction.' These areas can be summarized as focusing on: customer convenience/service and customer success.

With respect to customer convenience, as stated previously, both of the balanced firms had websites whereas neither quality-oriented firm had a website. While this fact alone may say little about the firms, it is their apparent views of the purpose or function of a website that helps shed light on their differences. Both quality-oriented firms stated that they did not have a website since they did not have the capacity to take on new business (at least prior to the current recession):

"We've always been local and had all the work we could handle with local. So why would we want a website?"

Case firm $\mathrm{QO}_{2}$

Hence, the quality-oriented firms appeared to view the purpose of a website as primarily focused on attracting new customers. Of course, the two balanced firms also viewed their websites as a means to attract new customers. However, this was not the sole purpose for the website. Balanced firms' websites also served as a place for existing customers to be able to download documents such as architectural drawings, see videos of the firm's processes, enable contact with company personnel (outside 
normal business hours), etc. While neither balanced firm provided for 'e-commerce' (i.e., allowing customers to order products on their websites), they both talked about adding such capability. In short, in addition to serving as a tool to acquire new business, the balanced firms also used their websites as a proactive means to allow their customers "24-7" access. For example, case firm $B_{1}$ stated:

"It [the website] is a really useful tool for us - after you have a conversation on the phone, have a look at our website. And we really spend a lot of time making sure our website is updated, it's full, it's complete, so they can learn about any product and any process in the company. So we do... we use it a lot." Case firm $\mathrm{B}_{1}$

Also within the area of 'customer convenience' is the practice of developing standardized product lines. Both balanced firms talked about developing standard product lines. Of course, such standardization is commonplace in that it can lead to streamlined production and lower costs; and viewed from the company's point of view, it is difficult to make a case that such practices are a service to the customer. However, both balanced firms discussed how such standardization makes it easier for their customers to specify and order products:

“...we also have some equipment that makes [product lines] and we've got a group of customers that rely on us for those and it's working with them getting a standardized list... a list of standard products that we sell and a standard cost so they don't have to keep coming back to us for quotes. They just have a price list and then also communicating that, just because we've got the standard list, we can do anything in that realm. So, it's certainly customer-driven."

Case firm $\mathrm{B}_{1}$

"On initial bids, [company] will provide customers with four different price-level bids in three different wood species. These packages (our Bronze, Silver, Gold, and Platinum Packages) bundle materials and options in various price-points, better showing relative pricing of popular alternatives." Case firm $\mathrm{B}_{2}$ - from company website 
Also within the area of 'proactive customer interaction' is the balanced firms' focus on their customers' success, in addition to their own success. Balanced firms developed new products with the explicit goal of helping their customers to increase profits, gain market share, solve challenges, etc. And in addition to these emphases for new product development, balanced firms emphasized customer service with a focus on helping their customers be successful. Examples of quotes to support this idea of 'focusing on customer success' include:

"we solicited a lot of opinion from our [customers] - will you be able to sell more wood? It's one thing to say this is a great feature and our customers are going to love it. But if there wasn't a strong link to being able to sell more wood, then we've just added costs..."

Case firm $\mathrm{B}_{1}$

"we spent a lot of time going through the communication and understanding what our customers were saying, that did it... were these changes going to actually allow them to compete better and gain more market share?"

Case firm $\mathrm{B}_{1}$

"we gave them [dentists - a new customer segment] a presentation earlier on the solid surface counter because it's nonporous, it won't support bacterial growth." Case firm $\mathrm{B}_{2}$ - discussion of product innovation to help reduce a costly customer challenge

"when we deliver cabinets, we deliver a counter right there. The contractor doesn't have the lead time, he's not wasting 2 to 3 weeks or, when we're busy, 4 weeks." Case firm $\mathrm{B}_{2}$ - helping customers shorten lead times

Before summarizing the principal research findings, there are two additional points to be addressed. The first is related to the issue of causality. Causality is implied in any discussions of 'best practices.' That is, it is implicit that implementation of the practices in a firm will cause improvement in performance. However, no such assurance can be made based on this research. The research is 
descriptive rather than prescriptive in that it merely examined practices in use by different firms and reported on apparent differences assuming that those practices receiving more emphasis from balanced firms are the 'best practices.' There are certainly innumerable practices in use by these firms beyond those described here that have led to their differences in performance. In fact, key among these differences appears to be the differences in strategy (discussed below). In the absence of a longitudinal experiment where performance is examined before-and-after implementation of practices in an organization, appeals must be made to the strength of the 'chain-of-evidence.' Such evidence includes the findings from within- and cross-case analysis combined with support from the literature. In that regard, one additional appeal is made to the literature as evidence that practices described here are in fact 'best practices' that are likely to lead to improved performance. The specific quality management practices explored here came from the Malcolm Baldrige National Quality Award (MBNQA) criteria. These criteria are widely recognized as leading practices for high-performing organizations. And in fact, the stated purposes for the criteria are [61]:

- "to help improve organizational performance practices, capabilities, and results

- to facilitate communication and sharing of information on best practices among U.S. organizations of all types

- to serve as a working tool for understanding and managing performance and for guiding organizational planning and opportunities for learning" 
Therefore, while no explicit claims of causality can be made, there is strong evidence from theory and practice to suggest that the best practices reported here are likely to contribute to improved quality and innovation performance.

The second point is related to the author's expectation going into this research. That expectation was that there would be essentially two kinds of firms -1 ) those that were successfully achieving innovation performance (in addition to quality) and 2) those that were striving to (i.e., 'wished they were') achieve innovation performance. However in hindsight this was a naïve assumption. It was quite apparent that it was no accident or oversight that resulted in the quality-oriented firms' lack of focus on innovation. Interviewees at the quality-oriented case firms had nearly 50 years of combined managerial experience. Both these gentlemen discussed how they had let many so-called 'opportunities' related to innovation pass them by. Further, both professed that their low-tech, and low-risk, approach was critical to their success. While their competitors (those still in business) were struggling to pay off debt, these firms stated that, times were hard, but they were profitable and they believed they would remain so.

In conclusion the primary research findings may be stated as follows:

The first step a firm should take is to ensure it includes innovation performance as part of its competitive strategy. In short, a firm must first want to pursue innovation. If this is the case, findings of this research, which are supported by a 
diverse cross-section of the literature, suggest that the following are best practices in quality management for achieving quality and innovation performance:

- Strive to ensure proactive interactions with customers

- Senior managers should actively encourage change and implement a culture of improvement, learning, and innovation in pursuit of 'excellence'; innovation must be seen as a means to some other goal such as improved product quality.

- Ensure employees have opportunities to share in, and are encouraged to, help the organization implement changes

- Engage in an active competitive benchmarking program to measure performance against the 'best practice' in the industry.

From the most pragmatic standpoint, if a manager were to pose the question, "What one thing can we do to adapt our quality management practices so that we achieve innovation performance as well?", the strongest evidence appears to be related to customer relationship management practices. Specifically, the evidence suggests that practices that emphasize proactive interaction with customers are a good place to focus. Table 14 presents several specific practices related to 'proactive customer interaction' based on the firms examined in this research. 
Table 14. Recommended Practices for Proactive Customer Interaction

\begin{tabular}{|c|c|}
\hline Focus Area & Specific Practices \\
\hline \multirow[t]{2}{*}{$\begin{array}{l}\text { Customer } \\
\text { Convenience }\end{array}$} & $\begin{array}{l}\text { Websites - create websites with a focus not only on acquiring new } \\
\text { customers but also as a service to existing customers. The website } \\
\text { should enable customers to contact company personnel, download } \\
\text { documents such as product drawings and specifications and price } \\
\text { lists for standard products. In short, the website emphasizes } \\
\text { customer convenience - making it easy for customers to do business } \\
\text { with the firm, obtain needed information when it is needed, etc. }\end{array}$ \\
\hline & $\begin{array}{l}\text { Standardized product lines }- \text { this practice is closely related to that } \\
\text { above. While standardizing product lines is generally associated } \\
\text { with being an internally-focused (vs. customer-focused) activity, } \\
\text { such efforts can also serve as a convenience to customers as well, } \\
\text { e.g., to make it easier for their customers to place orders on-line. }\end{array}$ \\
\hline \multirow[t]{2}{*}{$\begin{array}{l}\text { Customer } \\
\text { Success }\end{array}$} & $\begin{array}{l}\text { New product development - typical objectives of firms engaged in } \\
\text { product innovation are to increase profits, acquire new customers, } \\
\text { and increase market share. However, firms that sell business-to- } \\
\text { business may also develop new products with a focus on ensuring } \\
\text { their customers' success. For example, firms interviewed in this } \\
\text { research asked if their new product features would help their } \\
\text { customers sell more products, gain market share, or solve common } \\
\text { (and costly) problems. }\end{array}$ \\
\hline & $\begin{array}{l}\text { Customer service }- \text { services to customers such as delivering a } \\
\text { complete product package (e.g., a cabinet manufacturer delivering a } \\
\text { countertop at the same time as the cabinets) may help reduce } \\
\text { customers' lead time. }\end{array}$ \\
\hline
\end{tabular}




\section{Chapter 5. Contributions}

This chapter presents a summary of the contributions of this research to the bodies of knowledge related to methods for case firm selection, quality management, engineering management, and systems science. These contributions are followed by a discussion of contributions with respect to managerial practice. The chapter concludes with a summary of the strengths and limitations of the research as well as suggestions for future research.

\section{Contributions to Research}

The research has contributed to methods used for case firm selection. Specifically, to the author's knowledge, it is the first study to use DEA to aid in case firm selection. The literature related to case firm selection is sparse; and several experts have noted the challenges related to selecting case firms. For example, as stated in the literature review, Yin simply encourages researchers to, “...have defined a set of operational criteria whereby candidates will be deemed qualified to serve as cases. Then you should select randomly from the qualified candidates..." [88] And Eisenhardt recommends that 'theoretical sampling' (vs. random sampling) is preferred. She provides several examples of how other researchers have selected case firms such as selecting polar examples, i.e., "...one case of clearly successful firm performance and one unsuccessful case" [86]. Such an approach is challenging in situations where 'successful' and 'unsuccessful' are not clear. Lastly, as recently as 2008, Seawright and Gerring state that “...choosing good cases for extremely small samples is a challenging endeavor... the question of case selection has received relatively little 
attention from scholars..." These authors propose several quantitative methods for case selection [90]. However, common to all of the approaches (Seawright and Gerring's as well as Yin's and Eisenhardt's) is the need for quantitative data on the target audience.

While collection of such data (i.e., surveying the target audience) is not a novel contribution of the present research, nor perhaps is the use of cluster analysis to organize respondents into groups with similar responses, the author is not aware of any other research where the combination of DEA and cluster analysis has been used to aid in the selection of case firms. In that regard, DEA served both as a data reduction technique (in this case, from 204 case firm candidates to 26) as well as to identify a unique sector of the respondents. For the latter point, firms identified as efficient by DEA are those that maximize their outputs (quality and innovation performance) while at the same time using the minimum inputs (quality management practices). In short, the efficient firms demonstrate focus - emphasis on some practices, lack of emphasis on others. Given the primary research focus on identifying best practices, without DEA, there was greater risk of failing to identify differences in practices. That is, the potential for examining firms that took a 'shotgun approach' to quality management practices was greater and therefore the potential for studying case firms that were more similar than different with respect to quality management practices. 
In short, regardless of the technique used, the main objective is to somehow reduce the pool of candidate firms to those that demonstrate some diversity in their application of the practices while simultaneously achieving high performance with respect to the specified outputs. DEA is uniquely suited to such an objective. And from the author's knowledge of the forest industry, there is some evidence that the technique was successful in case firm selection. Specifically, one of the balanced case firms is quite well known in the forest industry as an innovative firm.

With respect to quality management, the research has contributed to the body of knowledge by exploring one of the apparent next steps in the field. In particular, the literature suggests that the progression in quality-related research has been to:

1. Define the multiple dimensions of quality;

2. Describe the specific principles and practices of quality management;

3. Develop and validate constructs and instruments to measure extent of implementation of the principles and practices; and

4. Use these instruments to explore correlations between quality management and performance.

Hence, this research went beyond exploring correlations and examined the potential contributions of specific quality management practices to both quality and innovation performance. Findings have confirmed prior researchers' suggestions that specifically how a company implements TQM will influence the type of performance achieved; further, as discussed below, the findings here agree with those of prior research in some areas and disagree in others with respect to which practices may lead to innovation performance. 
With regards to engineering management, the primary contribution to the body of knowledge of this research has been to provide new insights into how practices generally associated with innovation management may be integrated with quality management practices. In particular, because the target industry sector (the forest industry) is composed primarily of small firms, and not generally known for innovation performance, the emphasis of the research was on how these firms might adapt existing quality management practices. This is especially the case given that it is very unlikely that a small wood products firm would establish an R\&D department or any such entity focused on innovation. Hence, the question is really, how can small firms manage innovation? And the answer to that question assumed by the present research is - by adapting practices they already have in place related to quality management. The question is therefore, which practices and how? The research has confirmed and provided new insights into prior researchers' findings related to the importance of top management ensuring company strategy and culture are aligned with goals, as well as the correlations between customer focus, employee empowerment, and innovation performance. At the same time, the research has called into question other researchers' findings (or speculations), e.g., that benchmarking is 'perilous' to innovation performance. Other findings that conflict with prior research include those suggesting that 'education \& training', as well as 'process \& strategic management' are positively correlated with innovation performance; at least one balanced case firm placed little emphasis on employee training, and none of the firms emphasized strategic management or process management. 
This research has also confirmed prior researchers' findings regarding the 'cross-fertilization' of quality and innovation and has gone further to describe how such interactions are manifested in two case firms. Specifically, the research has provided some specific examples of how firms may pursue product innovation in an effort to improve product quality and then pursue a process innovation as well to enable the manufacture of the product. Further, the process innovation may in turn lead to additional product innovations that capitalize on the firm's investment in new processing capabilities.

With respect to systems science, the 'systems approach' is foundational to the main topic of this research - quality management. In fact, one author defines TQM as "... an integrated system of principles and procedures whose goal is to improve the quality of an organization's goods and services" [38]. The research explored companies' quality management practices via a questionnaire developed based on the criteria from the Malcolm Baldrige National Quality Award (MBNQA). As shown in Figure 1, the MBNQA conceives of quality management using a 'systems perspective.' As stated in the award criteria:

"The Criteria have continually progressed toward a comprehensive, integrated systems perspective of overall organizational performance management... A systems perspective includes your senior leaders' focus on strategic directions and on your customers... A systems perspective also includes using your measures, indicators, core competencies, and organizational knowledge to build your key strategies...Thus, a systems perspective means managing your whole organization, as well as its components, to achieve success." (emphasis added)

[61] 
In fact, one could argue that the practices described in criteria for the MBNQA extend well beyond quality management to general management. However again, the criteria are intended to examine organizations as systems - their management practices, the integration of these practices, and the firm's interaction with other entities. The research has been conducted with these principles in mind in that the firms' overall management practices (organization as an integrated system) and performance served in the selection of case firms. Then several research propositions examined the practices from the viewpoint of integration of practices within the organization (Proposition 2) as well as the firms' interactions with customers, competitors and other entities (Proposition 3).

The research contributed to the systems science body of knowledge by demonstrating how data envelopment analysis (DEA) may be used to examine organizations from a systems perspective. DEA allowed for simultaneous consideration of firms' inputs and outputs, i.e., examining the organizations' practices and performance holistically. Given that there were multiple inputs (quality management practices) and multiple outputs (quality and innovation - both of which were multi-dimensional), DEA enabled identification of case firms by considering the organization as a complex system. Most of the prior research has examined quality performance from the perspective of conformance to specifications, e.g., product defect rates; innovation performance has often been measured via metrics such as number of patents or new products released. Measuring quality and innovation too narrowly fails to account for the contingency approach by which firms with differing 
strategies, cultures, and contexts pursue the various dimensions of quality and innovation, i.e., how the organization as an integrated system achieves a complex array of performance outputs.

The findings also contribute to the systems science body of knowledge with respect to the dynamics inherent in complex systems. Specifically, the finding related to 'cross-fertilization' between quality and innovation suggests the possibility of multiple feedback loops and interactions between endogenous and exogenous factors. In fact, this finding is a contribution related to both systems science and engineering management, as discussed above. From a systems science perspective, this finding provides insights into how practices related to product development and process improvement, combined with feedback from customers and company personnel may interact. For example one could conceive of a causal loop diagram linking the inspiration for a product quality improvement (i.e., from customer feedback), to an innovation in product design that addresses this need, to a process innovation required to produce the new product, to additional new products that capitalize on the process innovation. The 'exogenous feedback loop' (customers) may result in modifications to the product design, whereas endogenous feedback (e.g., from operating personnel) may result in modifications to the process (i.e., continuous process improvement $-\mathrm{a}$ foundational principle of TQM).

Lastly, it is interesting to note that the key findings strongly reinforce the notion of organizations as complex systems. In particular, most if not all functional 
areas of a firm (e.g., sales, finance, production, management, etc.) are impacted by the findings. The findings relate to a broad array of elements internal and external to the firm - how leaders work to influence company culture, the degree to which employees are empowered, and how the firm interacts with external audiences such as customers and competitors.

\section{Contributions to Practice}

The research questions proposed and addressed here were developed based on the author's experience providing technical assistance to wood products manufacturers in the area of quality management. Further, the author's role as director of the Oregon Wood Innovation Center (OWIC) at Oregon State University is to provide technical assistance, training, and publications that help foster innovation in the forest industry. As such, the focus on contributing to managerial practice has existed from the onset of the project. In fact, the key findings were disseminated to the industry via short 'research briefs' on the OWIC website and professional society presentations prior to the formal completion of the dissertation. And there are plans to present the findings via educational publications and workshops as part of the author's work assignment.

The primary contributions to practice are to provide managers with recommendations for adapting current quality management systems to have greater impact on both quality and innovation performance. These recommendations are supported by theory and based on practices used by actual companies. The use of case studies has enabled the accumulation of 'real-world' information on companies' 
practices and as such, should be very relevant and practical for industry managers. The author's experience working with industry audiences is that busy managers need practical advice, 'step-by-step instructions', and place high emphasis on practices grounded in real-world experience. While much of the findings aren't readily transferable as simple step-by-step instructions, they do provide guidance to managers to know where to focus their efforts. Further, the findings suggest opportunities for further case research exploring some of the practices (e.g., 'proactive customer interaction') to provide additional details and examples on what specific actions companies can take.

Last but not least, given the critical importance of quality and innovation in today's competitive business environment, the results of this research will fill an important niche in the current management practices in these areas.

\section{Strengths and Limitations}

A key strength of this research is related to the timeliness and relevance of the topic. While there is significant research exploring connections between quality management and quality performance or general business performance, the research on connections to innovation performance is in its infancy. In fact, during the time when this research was being conducted, the American Society for Quality (ASQ - the leading professional society for quality professionals) titled its 2007 World Conference on Quality Improvement "Fueling Innovation through People and Quality.” Furthermore, a new international journal was established on the topic; in 
2009, the first edition of the International Journal of Quality and Innovation was

published. And as further evidence of the timeliness of the topic, the majority of the research that has empirically tested relationships between quality and innovation has been published in the last 10 years (which is ironically, when the author began this research). This research has helped to further our knowledge of not simply whether or not quality and innovation are related, but on which of the many quality management practices may be related to innovation performance.

While many of the strengths and limitations of the research have been addressed previously, the information is briefly summarized here. Strengths of the research include:

- The topic is timely as evidenced by the increasing attention given in the literature to studies exploring the relationships between quality management and innovation performance. And as stated above, the creation of a journal specifically focused on the topic International Journal of Quality and Innovation) provides additional justification for the timeliness of the subject matter.

- The research takes a broad, systems-based view of quality management practices. Much of the prior research on the topic continues to view quality management primarily from the standpoint of continuous improvement.

- Quality management, quality performance, and innovation performance are all addressed via multiple dimensions. This approach is well-grounded in the literature - experts have stated consistently that quality and innovation are inherently multidimensional. Even so, much prior research has perceived of the constructs narrowly such as defect rates as the proxy for quality and number of new products released for innovation.

- The proposed approach is based on a comprehensive review of the literature on quality management and measurement of performance.

- The establishment of best practices is the natural next step to be taken in the field. Several researchers have documented positive correlations between 
quality management and quality and innovation performance. However, there has been very little work focused on describing the specific practices that lead to superior performance.

- The combined use of quantitative analysis (DEA followed by cluster analysis) combined with qualitative analysis capitalizes on the strengths of both forms of research. DEA and cluster analysis aid in the selection of case firms and the quantitative approach overcomes issues related to researcher bias in case firm selection. This combined with the case study method adds breadth and depth to the findings and ensures the findings are grounded in actual practice.

- The use of DEA to aid in case firm selection is not only a unique contribution of the research, but is also aligned well with the subject matter of TQM. DEA is a valuable tool used in benchmarking. Given that benchmarking is a key quality management (and innovation) practice, it seems natural to use DEA to identify best practices within TQM.

Limitations of the research include:

- Descriptive vs. prescriptive - as discussed in the Results chapter, the research is descriptive in nature and thus no claims can be made that the best practices presented here will 'cause' improved performance. However, the strongest appeal that can be made is to the fact that the practices were derived from the Malcolm Baldrige National Quality Award criteria and as such are wellestablished in theory and in practice by high-performing organizations.

- Low response rate to the questionnaire - like many other research projects involving surveys, this project suffered from the continuing decline in response rate. Declining response rates are a recognized challenge in the research literature. Further compounding this issue however was the ongoing global recession at the time of data collection and the particular impact on the target industry. A few survey respondents returned uncompleted surveys and included a letter saying they were no longer in business due to the economy; many other surveys were returned as undeliverable (reason unknown). And as one potential interviewee stated in declining to be interviewed, "You couldn't have picked a worse time to do research in this industry; we're scrambling just to keep the doors open."

- Focus on single industry sector and region - limitations to generalizability to a broad manufacturing sector are acknowledged given the focus on a single industry sector, and even a limited region of the U.S. (West Coast). Further, there was lack of adequate representation from some of the sub-sectors (e.g., composites and secondary manufacturers). However, comparison of results to 
existing literature served to confirm many of the findings thus providing some assurance of generalizability.

- Small firms - all of the case firms had 75 or fewer employees. While these small firms are quite representative of the target industry, it is likely that the findings may not be applicable to larger firms.

- Narrow definition of 'performance' - there were no explicit steps taken to ensure the firms were actually successful in terms of profitability. This was by design in that the focus was on quality and innovation performance, not financial performance. However, the reported 'best practices' are not directly linked to profitability. Although, as previously stated, all of the firms discussed how challenging it was to stay in business and cited reasons they felt they were currently remaining competitive (e.g., focus on new markets, choosing not to invest in technology, etc.)

- Subjective data - with any survey data, there is the risk of respondents not reporting things accurately. Even with the interviews, respondents may tell you what they think you want to hear, i.e., give the 'textbook response' to a question on employee empowerment, for example. Hence, there can be challenges with internal validity. Steps taken to overcome this limitation include using DEA and use of multiple sources (triangulation) in the cases. DEA served to filter out those respondents that circled "all 5's" (high emphasis/performance on everything) in that such firms were not deemed efficient. And the interviews served to validate the questionnaire responses which in some cases, were not validated (e.g., no evidence found to support a respondent's high score on a certain practice).

Finally, with regards to DEA, an additional limitation of the research is related to the relatively non-traditional approach to the use of DEA. Rather than more conventional cost inputs and product outputs, ‘extent of TQM implementation' served as the inputs and perceptual measures of quality and innovation performance as the outputs. The author was only able to identify such an approach for using DEA by two other researchers - Forker et al. [38, 84] and Yoo [85]. Therefore, despite being 'nontraditional' with respect to DEA, there is support in the literature for such an approach. 
However, the most appropriate model for assessing the impact of TQM on performance is to consider actual TQM inputs. Ideally, these inputs would involve objective quantities such as 'dollars spent on the TQM program.' Of course, such data are not available as TQM is not a 'line-item expense' but rather is intended to be integrated throughout the operations of an organization. The literature review demonstrates that numerous researchers have come to the conclusion that subjective measures of TQM principles and practices are the only effective means to measure extent of TQM implementation [46, 50, 60, 67, 70].

\section{Suggestions for Future Research}

This research has revealed several topics where additional research is recommended based on either key findings of the present research and/or the limitations discussed above. Each topic is presented below.

As is always the case for descriptive research, additional studies should be conducted to validate the findings presented here. In particular, the research would involve a longitudinal study of a case firm (or firms) that are currently identified as quality-oriented, but which desire to improve their innovation performance. The study would measure their performance before and after implementation of several of the best practices reported here.

One of the key findings of the research is related to the importance of proactive customer interaction. While the case firms provided some details and examples of 
how to implement this practice, more research is needed to validate this finding and to provide greater depth. For example, validation may occur by comparing customer relationship management practices between innovative and non-innovative firms. Questions that may be addressed include, what is the nature of the proactive interaction, how do different companies acquire information from customers and how and to whom is it communicated within the firm? In fact, such research is currently underway in that at the time of this writing, the author has a graduate student exploring precisely these questions.

One finding that was not presented above, but for which further research is suggested, is with regards to how the firms defined quality. Based on Garvin's five definitions of quality [55], there were indications that the quality-oriented firms viewed quality based on either the product-based (e.g., some measurable product attribute such as $\mathrm{QO}_{2}$ 's emphasis on using the highest lumber grade) or user-based definition (such as $\mathrm{QO}_{1}$ 's statement that quality is perceived by the customer). However, both balanced firms appeared to use the value-based definition of quality; interestingly, the value-based definition is essentially one that balances excellence at a fair price (the customer view) with conformance to specifications at a reasonable cost (the firm view). Additional research may reveal the extent to which a company's conception of quality correlates to their focus on innovation.

In addition, given that many equate TQM with process management and continuous improvement, or even more narrowly as synonymous with the use of 
statistical process control (SPC), one surprising finding of the research is in the low emphasis all the case firms placed on these practices, and in particular the lack of use of tools such as SPC. This finding begs the question - "how exactly are these firms focused on product quality if not through such methods?" Additional research may shed light on this question.

Another potential area of additional research is related to financial performance of the firms. A study similar to this one could be conducted that also takes into account financial performance of the firms to ensure that the 'best practices' are in fact in some way also related to firm profitability.

Experts in innovation in the forest industry have recommended that firms focus more on product and business systems innovation, and less on process innovation [101]. Therefore, the findings regarding similarities in practices related to business systems innovation between the quality-oriented and balanced case firms was unexpected. Based on the interviews, it seems there may be some confusion on what specifically qualifies as a business systems innovation; interviewees generally needed little additional explanation to discuss product or process innovation, however most interviewees required clarification to understand what was meant by the questions related to business systems innovation. Also, it could be that the questionnaire items related to business systems innovation were too generic and thus did not differentiate between firms. Further research specifically focused on this form of innovation may help to confirm or disconfirm the findings of the present research. 
One final area where additional research is recommended is with regards to the finding related to 'cross-fertilization' between quality and innovation. Such research might focus on developing a system dynamics model showing the interrelationships between product quality, process innovation, and product innovation, while also taking into account internal (to the firm) influences such as related to process management/improvement and external influences such as customer feedback. 


\section{References}

[1] E. L. Grant and R. S. Leavenworth, Statistical Quality Control, Sixth ed. NY: McGraw-Hill, 1988.

[2] J. R. Evans and W. M. Lindsay, The Management and Control of Quality, Fifth ed. Cincinnati: South-Western Publishing, 2002.

[3] "Quality programs show shoddy results," in Wall Street Journal, ed. New York, 1992.

[4] E. E. Adam Jr., "Alternative quality improvement practices and organization performance," Journal of Operations Management, vol. 12, pp. 27-44, October 1994.

[5] O. Harari, "Ten reasons TQM doesn't work," Management Review, vol. 86, pp. 38-44, January 1997.

[6] K. McManus, "Is quality dead?," IIE Solutions, vol. 31, pp. 32-35, July 1999.

[7] U. Nwabueze, "An industry betrayed: The case of total quality management in manufacturing," The TQM Magazine, vol. 13, pp. 400-408, 2001.

[8] P. M. Senge, "Quality management: Current state of the practice," presented at the American Quality Congress, 1993.

[9] M. Hammer and J. Champy, Reengineering the Corporation: A Manifesto for Business Revolution. New York, NY: HarperCollins, 2001.

[10] H. J. Harrington, "Performance improvement: the rise and fall of reengineering," The TQM Magazine, vol. 10, pp. 69-71, 1998. 
[11] H. D. Allender, "Is reengineering compatible with total quality management?," Industrial Engineering, vol. 26, pp. 41-44, 1994.

[12] Y. F. Jarrar and E. M. Aspinwall, "Integrating total quality management and business process re-engineering: is it enough?," Total Quality Management \& Business Excellence, vol. 10, pp. 584-593, 1999.

[13] K. R. Bhote, The Ultimate Six Sigma: Beyond Quality Excellence to Total Business Excellence. New York: AMACOM - American Management Association, 2002.

[14] P. Pande and L. Holpp, What is Six Sigma? New York: McGraw-Hill, 2002.

[15] B. Klefsjö, H. Wiklund, and R. L. Edgeman, "Six sigma seen as a methodology for total quality management," Measuring Business Excellence, vol. 5, pp. 31$35,2001$.

[16] S. L. Ahire and D. Y. Golhar, "Quality management in large vs. small firms: An emperical investigation," Journal of Small Business Management, pp. 1-13, April 1996.

[17] S. L. Ahire, M. A. Waller, and D. Golhar, "Quality management in TQM versus non-TQM firms: An empirical investigation," International Journal of Quality \& Reliability Management, vol. 13, pp. 8-27, 1996.

[18] H. M. Beheshti and J. G. Lollar, "An empirical study of US SMEs using TQM," TQM \& Business Excellence, vol. 14, pp. 839-847, October 2003.

[19] G. S. Easton and S. L. Jarrell, "The effects of total quality management on corporate performance: An empirical investigation," Journal of Business, vol. 71, pp. 253-307, 1998. 
[20] D. I. Prajogo and A. Brown, "The relationship between TQM practices and quality performance and the role of formal TQM programs: An Australian empirical study," Quality Management Journal, vol. 11, pp. 31-42, 2004.

[21] C. B. Fotopoulos and E. L. Psomas, "The impact of "soft" and "hard" TQM elements on quality management results," International Journal of Quality \& Reliability Management, vol. 26, pp. 150-163, 2009.

[22] T. Kekale and J. Kekale, "A mismatch of cultures: A pitfall of implementing a total quality approach," International Journal of Quality \& Reliability Management, vol. 12, pp. 210-220, 1995.

[23] R. McAdam, G. Armstrong, and B. Kelly, "Investigation of the relationship between total quality and innovation: A research study involving small organisations," European Journal of Innovation Management, vol. 1, pp. 139147, 1998.

[24] S. B. Sitkin, K. M. Sutcliffe, and R. G. Schroeder, "Distinguishing control from learning in Total Quality Management: A contingency perspective," Academy of Management Review, vol. 19, pp. 537-564, 1994.

[25] D. I. Prajogo and A. S. Sohal, "The relationship between TQM practices, quality performance, and innovation performance: An empirical examination," International Journal of Quality \& Reliability Management, vol. 20, pp. 901918, 2003.

[26] T. Hill, Manufacturing Strategy - The Strategic Management of the Manufacturing Function. London: Macmillan Press, 1985. 
[27] R. G. Cooper, "Overhauling the new product process," Industrial Marketing Management, vol. 25, pp. 465-482, 1996.

[28] R. E. Cole and T. Matsumiya, "Too much of a good thing? Quality as an impediment to innovation," California Management Review, vol. 50, pp. 7793, Fall 2007.

[29] R. E. Cole and T. Matsumiya, "When the pursuit of quality risks innovation," The TQM Journal, vol. 20, pp. 130-142, 2008.

[30] D. I. Prajogo and A. S. Sohal, "TQM and innovation: A literature review and research framework," Technovation, vol. 21, pp. 539-558, 2001.

[31] H. E. Samaha, "Overcoming the TQM barrier to innovation," HR Magazine, vol. 41, pp. 145-149, June 1996.

[32] R. Sethi and A. Sethi, "Can quality-oriented firms develop innovative new products?," Journal of Product Innovation Management, vol. 26, pp. 206-221, 2009.

[33] P. J. Singh and A. J. R. Smith, "Relationship between TQM and innovation: An empirical study," Journal of Manufacturing Technology Management, vol. 15, pp. 394-401, 2004.

[34] G. K. Kanji, "Can Total Quality Management help innovation?," Total Quality Management, vol. 7, pp. 3-9, 1996.

[35] D. I. Prajogo and A. S. Sohal, "The integration of TQM and technology/ R\&D management in determining quality and innovation performance," Omega: The International Journal of Management Science, vol. 34, pp. 296-312, 2006. 
[36] M. Demirbag, E. Tatoglu, M. Tekinkus, et al., "An analysis of the relationship between TQM implementation and organizational performance: Evidence from Turkish SMEs," Journal of Manufacturing Technology Management, vol. 17, pp. 829-847, 2006.

[37] J. Feng, D. I. Prajogo, K. C. Tan, et al., "The impact of TQM practices on performance: A comparative study between Australian and Singaporean organizations," European Journal of Innovation Management, vol. 9, pp. 269278, 2006.

[38] L. B. Forker, D. Mendez, and J. C. Hershauer, "Total quality management in the supply chain: What is its impact on performance?," International Journal of Production Research, vol. 35, pp. 1681-1701, 1997.

[39] L. B. Forker, S. K. Vickery, and C. L. M. Droge, "The contribution of quality to business performance," International Journal of Operations \& Production Management, vol. 16, pp. 44-62, 1996.

[40] K. B. Hendricks and V. R. Singhal, "Does implementing an effective TQM program actually improve operating performance? Empirical evidence from firms that have won quality awards," Management Science, vol. 43, pp. 12581274, September 1997.

[41] K. B. Hendricks and V. R. Singhal, "The long-run stock price performance of firms with effective TQM programs," Management Science, vol. 47, pp. 359368, March 2001. 
[42] H. Kaynak, "Measuring organisational effectiveness and business performance in firms implementing total quality management," International Journal of Manufacturing Technology \& Management, vol. 8, pp. 355-381, 2006.

[43] D. I. Prajogo, "The comparative analysis of TQM practices and quality performance between manufacturing and service firms," International Journal of Service Industry Management, vol. 16, pp. 217-228, 2005.

[44] D. I. Prajogo and A. Brown, "Approaches to adopting quality in SMEs and the impact on quality management practices and performance," Total Quality Management, vol. 17, pp. 555-566, June 2006.

[45] D. I. Prajogo and A. S. Sohal, "The relationship between organization strategy, total quality management (TQM), and organization performance - the mediating role of TQM," European Journal of Operational Research, vol. 168, pp. $35-50,2006$.

[46] D. Samson and M. Terziovski, "The relationship between total quality management practices and operational performance," Journal of Operations Management, vol. 17, pp. 393-409, 1999.

[47] R. L. Shrivastava, R. P. Mohanty, and R. R. Lakhe, "Linkages between Total Quality Management and organisational performance: An empirical study for Indian industry," Production Planning and Control, vol. 17, pp. 13-30, January 2006.

[48] W. G. Lewis, K. F. Pun, and T. R. M. Lalla, "Exploring soft versus hard factors for TQM implementation in small and medium-sized enterprises," 
International Journal of Productivity and Performance Management, vol. 55, pp. 539-554, 2006.

[49] W. G. Lewis, K. F. Pun, and T. R. M. Lalla, "Empirical investigation of the hard and soft criteria of TQM in ISO 9001 certified small and medium-sized enterprises," International Journal of Quality \& Reliability Management, vol. 23, pp. 964-985, 2006.

[50] S. L. Ahire, D. Golhar, and M. A. Waller, "Development and validation of TQM implementation constructs," Decision Sciences, vol. 27, pp. 23-56, Winter 1996.

[51] P. G. Benson, J. V. Saraph, and R. G. Schroeder, "The effects of organizational context on quality management: An empirical investigation," Management Science, vol. 37, pp. 1107-1124, September 1991.

[52] A. Hovgaard and E. Hansen, "Innovativeness in the forest products industry," Forest Products Journal, vol. 54, pp. 26-33, January 2004.

[53] T. R. Anderson and G. P. Sharp, "A new measure of baseball batters using DEA," Annals of Operations Research, vol. 73, pp. 141-155, 1997.

[54] Anonymous. (2006, October 11). Quality Glossary. Available: http://www.asq.org/glossary/q.html

[55] D. A. Garvin, "What does "product quality" really mean?," Sloan Management Review, pp. 25-43, 1984.

[56] W. A. Shewhart, Economic Control of Quality of Manufactured Product, 50th Anniversary Commemorative Reissue (ASQC 1980) ed. Milwaukee, WI: American Society for Quality Control, 1931. 
[57] D. A. Garvin, "Quality on the line," Harvard Business Review, pp. 64-75, September-October 1983.

[58] NBC, "If Japan Can, Why Can't We?," in NBC White Paper, ed: CC-M, 1980.

[59] H. H. Goldman, "The origins and development of quality initiatives in American business," The TQM Magazine, vol. 17, pp. 217-225, 2005.

[60] B. B. Flynn, R. G. Schroeder, and S. Sakakibara, "A framework for quality management research and an associated measurement instrument," Journal of Operations Management, vol. 11, pp. 339-366, 1994.

[61] Anonymous, "Criteria for Performance Excellence, Malcolm Baldrige National Quality Program," National Institute of Standards and Technology2009.

[62] S. Black and L. J. Porter, "An empirical model for total quality management," Total Quality Management, vol. 6, pp. 149-164, 1995.

[63] S. L. Ahire, R. Landeros, and D. Y. Golhar, "Total quality management: A literature review and an agenda for future research," Production and Operations Management, vol. 4, pp. 277-306, Summer 1995.

[64] A. Wilkinson, "The other side of quality: 'soft' issues and the human resource dimension," Total Quality Management, vol. 3, pp. 323-329, May 1992.

[65] D. Dow, D. Samson, and S. Ford, "Exploding the myth: Do all quality management practices contribute to superior quality performance?," Production and Operations Management, vol. 8, pp. 1-27, Spring 1999.

[66] Anonymous, "Criteria for Performance Excellence, Malcolm Baldrige National Quality Program," National Institute of Standards and Technology2006. 
[67] J. V. Saraph, P. G. Benson, and R. G. Schroeder, "An instrument for measuring the critical factors of quality management," Decision Sciences, vol. 20, pp. 810-829, 1989.

[68] J. F. Hair Jr., W. C. Black, B. J. Babin, et al., Multivariate Data Analysis, 6th ed. Upper Saddle River, NJ: Pearson Prentice Hall, 2006.

[69] J. C. Nunnally, Psychometric Theory. New York, NY: McGraw-Hill, 1967.

[70] P. J. Singh and A. Smith, "An empirically validated quality management measurement instrument," Benchmarking: An International Journal, vol. 13, pp. 493-522, 2006.

[71] J. Motwani, "Critical factors and performance measures of TQM," The TQM Magazine, vol. 13, pp. 292-300, 2001.

[72] J. R. Cooper, "A multidimensional approach to the adoption of innovation," Management Decision, vol. 36, pp. 493-502, 1998.

[73] F. Betz, Managing Technological Innovation: Competitive Advantage from Change. New York: John Wiley \& Sons, Inc., 1998.

[74] F. Damanpour, "Organizational complexity and innovation: Developing and testing multiple contingency models," Management Science, vol. 42, pp. 693$716,1996$.

[75] E. M. Rogers, Diffusion of Innovations, Fourth ed. New York: The Free Press, 1995.

[76] J.-A. Johannessen, B. Olsen, and G. T. Lumpkin, "Innovation as newness: What is new, how new and new to whom?," European Journal of Innovation Management, vol. 4, pp. 20-31, 2001. 
[77] J. M. Utterback, Mastering the Dynamics of Innovation: How Companies Can Seize Opportunities in the Face of Technological Change. Boston, MA: Harvard Business School Press, 1994.

[78] J. M. Utterback and W. J. Abernathy, "A dynamic model of process and product innovation," Omega: The International Journal of Management Science, vol. 3, pp. 639-656, 1975.

[79] F. Damanpour, "Organizational innovation: A meta-analysis of effects of determinants and moderators," Academy of Management Journal, vol. 34, pp. 555-590, 1991.

[80] L.-H. Lin and I.-Y. Lu, "Product quality as a determinant of product innovation: An empirical analysis of the global automotive industry," Total Quality Management, vol. 17, pp. 141-147, March 2006.

[81] L. Diaz-Balteiro, A. C. Herruzo, M. Martinez, et al., "An analysis of productive efficiency and innovation activity using DEA: An application to Spain's wood-based industry," Forest Policy and Economics, vol. 8, pp. 762773, 2006.

[82] D. I. Prajogo and A. S. Sohal, "Transitioning from total quality managment to total innovation management: An Australian case," International Journal of Quality \& Reliability Management, vol. 21, pp. 861-875, 2004.

[83] A. Charnes, W. W. Cooper, and E. Rhodes, "Measuring the efficiency of decision making units," European Journal of Operational Research, vol. 2, pp. 429-444, 1978. 
[84] L. B. Forker and D. Mendez, "An analytical method for benchmarking best peer suppliers," International Journal of Operations \& Production Management, vol. 21, pp. 195-209, 2001.

[85] H. Yoo, "A study on the efficiency evaluation of total quality management activities in Korean companies," Total Quality Management, vol. 14, pp. 119$128,2003$.

[86] K. M. Eisenhardt, "Building theories from case study research," Academy of Management Review, vol. 14, pp. 532-550, 1989.

[87] R. K. Yin, "The case study crisis: Some answers," Administrative Science Quarterly, vol. 26, pp. 58-65, 1981.

[88] R. K. Yin, Case Study Research: Design and Methods, Second ed. Thousand Oaks, CA: Sage Publications Inc., 1994.

[89] B. G. Glaser and A. L. Strauss, The Discovery of Grounded Theory; Strategies for Qualitative Research. Chicago, IL: Aldine Publishing Company, 1967.

[90] J. Seawright and J. Gerring, "Case selection techniques in case study research: A menu of qualitative and quantitative options," Political Research Quarterly, vol. 61, pp. 294-308, 2008.

[91] R. Campbell and C. A. Ahrens, "Innovative community services for rape victims: An application of multiple case study methodology," American Journal of Community Psychology, vol. 26, pp. 537-571, 1998.

[92] H. J. Harrington, "The productivity and quality connection," IEEE Journal on Selected Areas in Communication, vol. SAC-4, pp. 1009-1014, October 1986. 
[93] B. B. Flynn, R. Schroeder, and S. Sakakibara, "Determinants of quality performance in high- and low-quality plants," Quality Management Journal, vol. 2, pp. 8-25, Winter 1995.

[94] A. Schuler and U. Buehlmann, "Identifying Future Competitive Business Strategies for the U.S. Residential Wood Furniture Industry: Benchmarking and Paradigm Shifts," USDA Forest Service, Northeastern Research Station, Newtown Square, PA, General Technical Report GTR-NE-304, March 2003.

[95] A. Schuler, R. Taylor, and P. Araman, "Competitiveness of U.S. wood furniture manufacturers: Lessons learned from the softwood moulding industry," Forest Products Journal, vol. 51, pp. 14-20, July/ August 2001.

[96] M. Anderson and A. S. Sohal, "A study of the relationship between quality management practices and performance in small businesses," International Journal of Quality \& Reliability Management, vol. 16, pp. 859-877, 1999.

[97] S. Mathiyalakan and C. Chung, "A DEA approach for evaluating quality circles," Benchmarking for Quality Management \& Technology, vol. 3, pp. 5970, 1996.

[98] K. P. Triantis and R. McNelis, "The measurement and empirical evaluation of quality productivity for a manufacturing process: A data envelopment analysis (DEA) approach," in Flexible Automation and Intelligent Manufacturing, Stuttgart, Germany, 1995, pp. 1134-1145.

[99] Y. K. Suk, "Measuring the impact of total quality management (TQM) on efficiency using data envelopment analysis (DEA) in the hospital industry: The 
case of the east south central region of the United States," Ph.D., School of Business Administration, University of Mississippi, University, 1998.

[100] B. A. G. Bossink, "The strategic function of quality in the management of innovation," Total Quality Management, vol. 13, pp. 195-205, 2002.

[101] E. Hansen, S. Korhonen, E. Rametsteiner, et al., "Current state-of-knowledge: Innovation research in the global forest sector," Journal of Forest Products Business Research, vol. 3, p. 32, 2006.

[102] J. S. Peters, D. T. Damery, and P. Clouston, "A decade of innovation in particleboard and composite materials: A content analysis of Washington State University’s International Particleboard/ Composite Materials Symposium proceedings," Journal of Forest Products Business Research, vol. 3, pp. 1-16, 2006.

[103] M. J. Benner and M. L. Tushman, "Exploitation, exploration, and process management: The productivity dilemma revisited," Academy of Management Review, vol. 28, pp. 238-256, 2003.

[104] D. T. Hoang, B. Igel, and T. Laosirihongthong, "The impact of total quality management on innovation: Findings from a developing country," International Journal of Quality \& Reliability Management, vol. 23, pp. 1092$1117,2006$.

[105] D. I. Prajogo and A. S. Sohal, "The multidimensionality of TQM practices in determining quality and innovation performance - an empirical examination," Technovation, vol. 24, pp. 443-453, 2004. 
[106] D. I. Prajogo and P. Ahmed, K., "The relationships between quality, innovation and business performance: An empirical study," International Journal of Business Performance Management, vol. 9, pp. 380-405, 2007.

[107] J. S. Armstrong and T. S. Overton, "Estimating nonresponse bias in mail surveys," Journal of Marketing Research, vol. 14, pp. 396-402, 1977.

[108] R. D. Banker, A. Charnes, and W. W. Cooper, "Some models for estimating technical and scale inefficiencies in data envelopment analysis," Management Science, vol. 30, pp. 1078-1092, 1984.

[109] H. Scheel. (2000). Efficiency Measurement System (Version 1.3.0). Available: http://www.holger-scheel.de/ems/

[110] R. D. Banker, A. Charnes, W. W. Cooper, et al., "An introduction to data envelopment analysis with some of its models and their uses," in Research in Governmental and Nonprofit Accounting. vol. 5, J. L. Chan and J. Patton, Eds., ed Greenwich, CT: JAI Press, 1989, pp. 125-163.

[111] S. Sherrill. (2009, August 16th). Radiata Pine imports from New Zealand threatened. Available: http://www.risiinfo.com/blogs/Radiata-Pine-importsfrom-New-Zealand-threatened.html?source=rss

[112] U. Buehlmann and A. Schuler, "The U.S. household furniture industry: Status and opportunities," Forest Products Journal, vol. 59, pp. 20-28, 2009.

[113] Anonymous. (2010, August 16th). Furniture and Related Products: Customs Value by Customs Value for ALL Countries. Available: http://ita.doc.gov/td/ocg/imp337.htm 
[114] B. Jacklet. (2010, August 16). Hard times for the big boys. Available: http://www.oregonbusiness.com/ben/3746-hard-times-for-the-big-boys

[115] Anonymous. (2010, August 16). New Privately Owned Housing Units Started in the United States, by Intent and Design. Available: http://www.census.gov/const/startsusintenta.pdf

[116] S. Zika. (2009, August 16). Available: http://www.hamptonaffiliates.com/ArDisplay.aspx?ID=94\&SecID=47

[117] P. Crespell, C. Knowles, and E. Hansen, "Innovativeness in the North American softwood samilling industry," Forest Science, vol. 52, pp. 568-578, 2006. 
Appendix A - Quality Constructs and Measurement Instruments 
Appendix A1 - Saraph et al.

Questions used for Measuring the Critical Factors of Quality Management [67]

Factor 1: Role of divisional top management and quality policy

1. Extent to which the top division executive (responsible for division profit and loss) assumes responsibility for quality performance.

2. Acceptance of responsibility for quality by major department heads within the division.

3. Degree to which divisional top management (top divisional executive and major department heads) is evaluated for quality performance.

4. Extent to which the division top management supports long-term quality improvement process.

5. Degree of participation by major department heads in the quality improvement process.

6. Extent to which the divisional top management has objectives for quality performance.

7. Specificity of quality goals within the division.

8. Comprehensiveness of the goal-setting process for quality within the division.

9. Extent to which quality goals and policy are understood within the division.

10. Importance attached to quality by the divisional top management in relation to cost and schedule objectives.

11. Amount of review of quality issues in divisional top management meetings.

12. Degree to which the divisional top management considers quality improvement as a way to increase profits.

13. Degree of comprehensiveness of the quality plan within the division.

Factor 2: Role of the quality department

1. Visibility of the quality department.

2. Quality department's access to divisional top management.

3. Autonomy of the quality department.

4. Amount of coordination between the quality department and other departments.

5. Effectiveness of the quality department in improving quality.

Factor 3: Training

1. Specific work-skills training (technical and vocational) given to hourly employees throughout the division.

2. Quality-related training given to hourly employees throughout the division.

3. Quality-related training given to managers and supervisors throughout the division.

4. Training in the 'total quality concept' (i.e., philosophy of company-wide responsibility for quality) throughout the division. 
5. Training in the basic statistical techniques (such as histograms and control charts) in the division as a whole.

6. Training in advanced statistical techniques (such as design of experiments and regression analysis) in the division as a whole.

7. Commitment of the divisional top management to employee training.

8. Availability of resources for employee training in the division.

Factor 4: Product/ service design

1. Thoroughness of new product/ service design reviews before the product/ service is produced and marketed.

2. Coordination among affected departments in the product/ service development process.

3. Quality of new products/ services emphasized in relation to cost or schedule objectives.

4. Clarity of product/ service specifications and procedures.

5. Extent to which implementation/ producibility is considered in the product/ service design process.

6. Quality emphasis by sales, customer service, marketing, and PR personnel.

Factor 5: Supplier quality management (supplier of goods and/or services)

1. Extent to which suppliers are selected based on quality rather than prices or schedule.

2. Thoroughness of the supplier rating system.

3. Reliance on reasonably few dependable suppliers.

4. Amount of education of supplier by division.

5. Technical assistance provided to the suppliers.

6. Involvement of the supplier in the product development process.

7. Clarity of specifications provided to suppliers.

Factor 6: Process management/ operating procedures

1. Use of acceptance sampling to accept/ reject lots of batches or work.

2. Amount of preventative (sic) equipment maintenance.

3. Extension to which inspection, review, or checking of work is automated.

4. Amount of incoming inspection, review, or checking.

5. Amount if in-process inspection, review, or checking.

6. Amount of final inspection, review, or checking of work.

7. Stability of production schedule/ work distribution.

8. Degree of automation of the process.

9. Extent to which process design is 'fool-proof' and minimizes the chance of employee errors.

10. Clarity of work or process instructions given to employees.

Factor 7: Quality data and reporting

1. Availability of cost of quality data in the division.

2. Availability of quality data (error rates, defect rates, scrap, defects, etc.) 
3. Timeliness of the quality data.

4. Extent to which quality data (cost of quality, defects, errors, scrap, etc.) are used as tools to manage quality.

5. Extent to which quality data are available to hourly employees.

6. Extent to which quality data are available to managers and supervisors.

7. Extent to which quality data are used to evaluate supervisor and managerial performance.

8. Extent to which quality data, control charts, etc. are displayed at employee work stations.

Factor 8: Employee relations

1. Extent to which quality circle or employee involvement type programs are implemented in the division.

2. Effectiveness of quality circle or employee involvement type programs in the division.

3. Extent to which employees are held responsible for error-free output.

4. Amount of feedback provided to employees on their quality performance.

5. Degree of participation in quality decisions by hourly/ nonsupervisory employees.

6. Extent to which quality awareness building among employees is ongoing.

7. Extent to which employees are recognized for superior quality performance

8. Effectiveness of supervisors in solving problems/ issues. 
Appendix A2 - Flynn et al.

Questions used for Measuring the Critical Factors of Quality Management [60]

(Note: The authors intentionally reverse the wording of some of the questions to keep survey respondents alert)

\section{Dimension I: Top Management Support}

Quality Leadership

1. All major department heads within our plant accept their responsibility for quality.

2. Plant management provides personal leadership for quality products and quality improvement.

3. The top priority is evaluating plant management in quality performance.

4. All major department heads within our plant work towards encouraging justin-time production.

5. Our top management strongly encourages employee involvement in the production process.

Quality Improvement Rewards

1. Workers are rewarded for quality improvement.

2. Supervisors are rewarded for quality improvement.

3. If I improve quality, management will reward me.

4. We pay a group incentive for quality improvement ideas.

5. Our plant has an annual bonus system based on plant productivity.

6. Nonfinancial incentives, such as jackets, coffee cups, etc. are used to reward quality improvement.

\section{Dimension II: Quality Information}

\section{Process Control}

1. Processes in our plant are designed to be 'fool proof.'

2. A large percent of the equipment or processes on the shop floor are currently under statistical quality control.

3. We make extensive use of statistical techniques to reduce variance in processes.

Feedback

1. Charts showing defect rates are posted on the shop floor.

2. Charts showing schedule compliance are posted on the shop floor.

3. Charts plotting the frequency of machine breakdowns are posted on the shop floor.

4. I am never told whether I am doing a good job.

5. Information on quality performance is readily available to employees.

6. My manager never comments about the quality of my work. 


\section{Dimension III: Process Management}

\section{Cleanliness and Organization}

1. Our plant emphasizes putting all tools and fixtures in their place.

2. We take pride in keeping our plant neat and clean.

3. Out plant is kept clean at all times.

4. I often have trouble finding the tools I need.

5. Our plant is disorganized and dirty.

\section{Dimension IV: Product Design}

New Product Quality

1. New product designs are thoroughly reviewed before the product is produced and sold.

2. Customer requirements are thoroughly analyzed in the new product design process.

3. Reducing the cost of new products is a more important priority than new product quality.

4. Schedule concerns are more important than quality in the new product development process.

Interfunctional Design Process

1. Direct labor employees are involved to a great extent (on teams or consulted) before introducing new products or making product changes.

2. Manufacturing engineers are involved to a great extent before the introduction of new products.

3. There is little involvement of manufacturing and quality people in the early design of products, before they reach the plant.

4. We work in teams, with members from a variety of areas (marketing, manufacturing, etc.) to introduce new products.

\section{Dimension V: Workforce Management}

\section{Selection for Teamwork Potential}

1. We use ability to work in a team as a criterion in employee selection.

2. We use problem solving ability as a criterion in selecting employees.

3. We use work values and ethics as a criterion in employee selection.

\section{Teamwork}

1. Our plant is organized into permanent production teams.

2. During problem solving sessions, we make an effort to get all team members' opinions and ideas before making a decision.

3. Our plant forms teams to solve problems.

4. In the past three years, many problems have been solved through small group sessions. 


\section{Dimension VI: Supplier Involvement}

Supplier Relationship

1. We strive to establish long-term relationships with suppliers.

2. Our suppliers are actively involved in our new product development process.

3. Quality is our number one criterion in selecting suppliers.

4. We rely on a small number of high quality suppliers.

\section{Dimension VII: Customer Involvement}

\section{Customer Interaction}

1. We frequently are in close contact with our customers.

2. Our customers seldom visit our plant.

3. Our customers give us feedback on quality and delivery performance. 
Appendix A3 - Ahire et al.

Questions used for Integrated Quality Management Constructs [50]

1. Top Management Commitment

- Top-level managers view quality as being more important than cost.

- Top-level managers view quality as being more important than meeting production schedules.

- Our performance evaluation by the top-level management depends heavily on quality.

- Top-level managers allocate adequate resources toward efforts to improve quality.

- We have clear quality goals identified by top-level managers.

- At company-wide meetings top-level managers often discuss the importance of quality.

2. Customer Focus

- Manufacturing managers are aware of the results of customer satisfaction surveys.

- A summary of customer complaints is given to manufacturing managers regularly.

- To achieve greater customer satisfaction, our company actively seeks ways to improve our primary product.

- Our company has been customer focused for the past two years.

3. Supplier Quality Management (Answer with respect to a critical component of the primary product)

- Quality is a more important criterion than price in selecting suppliers of the major component.

- Our supplier rating system considers the supplier's engineering capability.

- Our supplier rating system considers the supplier's financial stability.

- Our supplier rating system considers the supplier's delivery performance.

- We provide technical assistance to our suppliers of this component.

- We are more interested in developing a long-term relationship with these suppliers than reducing prices.

4. Design Quality Management

- Our design engineers are required to have some shop floor experience.

- Our design engineers are required to have some marketing experience.

- We use Taguchi methods extensively.

- We use error prevention techniques such as Shingo in designing the manufacturing process. 
- Quality Function Deployment (QFD) is used in the design of our primary product.

- There are engineers from other functional departments on a design team.

\section{Benchmarking}

- We are engaged in extensive benchmarking of competitors' products that are similar to our primary product.

- Our benchmarking activities have reduced costs.

- We have engaged in extensive benchmarking of other companies' business processes in other industries.

- Benchmarking has helped improve our product.

- We will definitely continue benchmarking.

6. SPC Usage

- $\mathrm{SPC}$ is used extensively in our plant.

- SPC has been effective in improving the quality of our primary product.

- We will continue to use SPC in the manufacture of our primary product.

- Production workers are well-trained in SPC.

7. Internal Quality Information Usage

- Scrap rates of our primary product are readily available.

- Rework rates of our primary product are readily available.

- Cost of quality data concerning our primary product is readily available.

- Quality information is displayed at most of the work stations.

- Progress toward quality-related goals is displayed in our plant.

- Information about defects is conveyed to the appropriate workstations.

8. Employee Empowerment

- Our line workers inspect the quality of their own work; inspection is not the responsibility of an inspector.

- Line workers are encouraged to fix problems they find.

- Line workers are given the resources necessary to correct quality problems they find.

- Line workers have technical assistance available to them to help them solve quality problems.

- A problem solving network is available to line workers in solving quality related problems.

9. Employee Involvement

- Cross-functional teams are often used. 
- All employee suggestions are evaluated.

- Most employee suggestions are implemented.

10. Employee Training

- Resources are available for employee quality training in our plant.

- There is almost always some kind of employee quality training going on in our plant.

- Plant managers are often involved in quality training.

- Most employees in our plant are trained to use quality problem solving techniques such as cause and effect diagrams.

- Most employees in our plant do not view each new quality seminar or training program as "just another fad."

11. Product Quality (Answer on a scale of 1 to 7 , where: $1=$ worst in industry, $4=$ average, $7=$ best in industry)

- The performance of our primary product is...

- The reliability of our primary product is...

- The conformance to engineering specifications of our primary product is...

- The durability of our primary product is...

12. Supplier Performance (Answer with respect to suppliers of the critical component of the primary product)

- Suppliers send us shipments of this component that conform to specifications.

- Most quality problems in the past have easily been resolved with our suppliers.

- Our suppliers are always eager to resolve quality problems.

- The critical component is durable.

- The critical component's performance is excellent.

- The critical component is reliable. 
Appendix A4 - Singh and Smith

Questions used for measurement of Quality Management [70]

1. Top Management Leadership

T1: Quality manager ensures quality system is continually improved

T2: Quality system regularly reviewed by management

T3: Internal quality audits verify effectiveness of quality system

T4: Statistical thinking reflected in policies/ processes/ reporting system

T5: CEO plays key role in creating values

T6: Values converted into practical policies and plans

T7: Customers contribute to development of values

T8: Suppliers had input into developing values

T9: Employees contribute to development of values

T10: Culture that CEO is creating is consistent with values

T11: Employees are responsible/ exercise leadership

T12: Employees know their roles and goals

T13: Changes to systems enable improvements

T14: Top management committed to quality

T15: Organization encourages participation of all stakeholders

T16: Top management accepts responsibility for quality

T17: There are sufficient personnel to manage quality-related activities

T18: Quality regarded as most important competitive priority

T19: Reward/ remuneration of parties based on quality of output

T20: Top management generates consensus on future direction

T21: Top management encourages long-term strategic thinking

2. Customers

$\mathrm{C} 1$ : Misunderstandings about customer orders are rare

$\mathrm{C} 2$ : All contracts are systematically reviewed

C3: Changes to contracts lead to lots of confusion

C4: Customers access appropriate persons to resolve complaints

C5: There are systematic processes for handling complaints

C6: Customer feedback improves customer relations, etc.

C7: Customers contribute to development of values

C8: Organization measures customer satisfaction

C9: Customers are encouraged to provide feedback

C10: Organization is aware of customer requirements

C11: Customers help design new products/ processes

C12: Processes/ activities increase customer satisfaction

C13: Customer satisfaction is a measure of quality

3. Employees

E1: Everyone is aware how quality policy affect his job

E2: Employees are responsible/ exercise leadership

E3: Employees know their roles and goals 
E4: Employees are fully trained for the work they perform

E5: HR plans integrated with overall plans/ values

E6: Individual employee development and motivation promoted

E7: Employees find their work very fulfilling

E8: Managing performance of employees improved flexibility/ responsiveness

E9: Recognition/ reward processes achieve goals

E10: Employees provided with feedback

E11: Employees aware of changes to performance measurement

E12: Employees freely communicate with others

E13: Communication system is effective

E14: Processes/ structures are in place to achieve OH\&S obligations

E15: Organization has "open" culture

E16: Employees work in teams

E17: Employees effect change to achieve objectives

E18: Employees have role in formulating plans

E19: Employees continuously improve work output

4. Suppliers

S1: Misunderstandings about orders placed with suppliers are rare

S2: All subcontractors suited to tasks they perform

S3: Materials from all customers/ suppliers treated same

S4: Quality of supplied products/ services are assessed

S5: Suppliers receive information to improve quality/ responsiveness

S6: Gains from cooperation with suppliers shared with them

S7: Quality is the main criterion for choosing suppliers

S8: Organization seeks assurance of quality from suppliers

S9: Long-term stable relationships with suppliers is sought

S10: Suppliers involved in development of new products

5. Information and Communication Systems

IC1: Quality manual cover all requirements for quality

IC2: Obsolete documents do not cause confusion with new versions

IC3: Possible to establish details of finished products

IC4: Possible to identify inspection status of materials

IC5: Quality manual is updated when processes change

IC6: Data collected is able to measure performance

IC7: Data is reliable and valid

IC8: Data collection promotes "management by facts"

IC9: Key data enhances understanding of issues

IC10: Statistical thinking reflected in policies/ processes/ reporting system

IC11: Data on quality is always timely

IC12: Data on quality widely shared

IC13: Employees provided with feedback

IC14: Data/ documents on quality readily available 


\section{Processes}

P1: Before starting job, plans for quality are produced

P2: Disciplined design process had led to improvements

P3: Products are checked against orders before delivery

P4: Products that cannot be tested are continuously monitored

P5: Equipment to test/ inspect is available

P6: Everyone is aware of what happens to products that fail inspections

P7: Reviews of all aspects are carried out

P8: If reviews indicate problems, actions are taken

P9: If problems occur, actions are taken

P10: Handling/ storage/ delivery methods minimize quality problems

P11: Products/ processes are inspected/ tested

P12: Innovation processes/ products/ services have been commercialized

P13: R\&D develop world-class techniques/ technologies

P14: Organization supports culture of creativity and innovation

P15: There is strong emphasis on internal customer/ supplier relationships

P16: Employees continuously improve work output

P17: Quality Assurance processes ensure customer requirements are met

P18: Strong emphasis is given on quality in design

P19: SPC techniques are used

P20: Physical work environment is safe for employees

P21: Employees have "zero-defects" mentality

7. Wider Community

W1: Organization included community responsibilities into policies

W2: Organization developed plans to manage risks to community

W3: Experience gained through best practice shared with community

8. Competitors

CP1: Organization benchmarks itself

CP2: There is keen competition in local and foreign markets

CP3: A few large competitors dominate the industry

9. Business Conditions (how is the organization currently being affected by the following business environmental factors)

$\mathrm{BC} 1$ : The costs of business inputs (e.g., labor, material, overheads)

$\mathrm{BC} 2$ : The availability of suitably qualified staff

$\mathrm{BC} 3$ : The industrial relations environment

BC4: Competition in local and foreign markets

BC5: The margins in the industry

BC6: The competitive structure of the industry

BC7: Customers' loyalty

$\mathrm{BC} 8$ : The rules and regulations that govern the industry

BC9: Ecological considerations in this industry

$\mathrm{BC} 10$ : The rate of introduction of new products and services 
$\mathrm{BC} 11$ : The rate of innovation of new operational processes

$\mathrm{BC} 12$ : The rate of change in taste and preference of customers 
Appendix A5 - Samson and Terziovski

Questions used for measurement of effectiveness of TQM implementation [46]

\section{A.1.1 Leadership}

Le1 Senior managers actively encourage change and implement a culture of trust, involvement and commitment in moving towards 'Best Practice' Le2 There is a high degree of unity of purpose throughout our site, and we have eliminated barriers between individuals and/ or departments Le3 'Champion(s) of change' are effectively used to drive 'Best Practice' at this site

Le4 At this site we proactively pursue continuous improvement rather than reacting to crisis 'fire-fighting'

Le5 Ideas from production operators are actively used in assisting management Le6 Environmental ('green') protection issues are proactively managed at this site

\section{A.1.2 People Management}

Pe1 The concept of the 'internal customer' (i.e., the next person or process down the line and including all employees) is well understood at this site $\mathrm{Pe} 2 \mathrm{We}$ have an organization-wide training and development process, including career path planning, for all our employees

Pe3 Our site has effective 'top-down' and 'bottom-up' communication processes

Pe4 Employee satisfaction is formally and regularly measured

Pe5 Our Occupational Health and Safety practices are excellent

Pe6 Employee flexibility, multi-skilling and training are actively used to support improved performance

Pe7 All employees believe that quality is their responsibility

\section{A.1.3 Customer focus}

Cfl We know our external customers' current and future requirements (both in terms of volume and product characteristics)

Cf2 These customer requirements are effectively disseminated and understood throughout the workforce

Cf3 In designing new products and services we use the requirements of domestic customers

Cf4 We have an effective process for resolving external customers' complaints Cf5 Customer complaints are used as a method to initiate improvements in our current processes

Cf6 We systematically and regularly measure external customer satisfaction

\section{A.1.4 Planning}

P11 We have a mission statement which has been communicated throughout the company and is supported by our employees 
P12 We have a comprehensive and structured planning process which regularly sets and reviews short and long-term goals

P13 Our plans focus on achievement of 'Best Practice'

P14 When we develop our plans, policies, and objectives we always incorporate customer requirements, supplier capabilities, and needs of other stakeholders, including the community

P15 We have a written statement of strategy covering all manufacturing operations which is clearly articulated and agreed to by our Senior Managers P16 Our site's manufacturing operations are effectively aligned with the central business mission

\section{A.1.5 Process management}

Qp1 Our suppliers work closely with us in product development

Qp2 We work closely with our suppliers to improve each others' processes Qp3 Our suppliers have an effective system for measuring the quality of the materials they send to us

Qp4 We have well established methods to measure the quality of our products and services

Qp5 We have site-wide standardized and documented operating procedures

\section{A.1.6 Information and analysis}

At this site we have undertaken benchmarking in the following areas:

Please circle as many numbers as are appropriate Yes $\quad$ No

(a) bm21

(a) bm22

Relative Cost Position

1

Operating Processes

(a) bm23

(a)bm24

Technology

Quality Procedures

12

(a)bm25

Customer Service

12

12

12

Estimate the total number of business days your site invests per year in reviewing the following information relating to other firms in your industry, where $1=$ None; $2=$ Less than three business days; 3 = Between three and five business days; 4 = Between six and twenty business days.

Please circle one number against each factor

$\mathrm{Bm} 5 \mathrm{c}$ Other firms' product quality and procedures $\begin{array}{lllll}1 & 2 & 3 & 4 & 5\end{array}$

Bm5d Other firms' human resource practices and policies

$\begin{array}{lllll}1 & 2 & 3 & 4 & 5\end{array}$

Bm5h Other firms' processes in bringing new products to market $1 \begin{array}{lllll}1 & 2 & 3 & 4 & 5\end{array}$

\section{A.1.7 Organizational performance}

Please indicate (by writing a single number, ranging from one through to five, in the vacant end column) your site's current performance level for EACH of the listed attributes. 


\begin{tabular}{|c|c|c|c|c|c|}
\hline $\begin{array}{l}\text { Dependent Variable } \\
\text { (performance outcom }\end{array}$ & 1 & 2 & 3 & 4 & 5 \\
\hline Po7a Customer satisfaction & $\begin{array}{l}\text { Sometimes } \\
\text { meets } \\
\text { expectations }\end{array}$ & $\begin{array}{l}\text { Generally } \\
\text { meet } \\
\text { expectations }\end{array}$ & $\begin{array}{l}\text { Consistently } \\
\text { meet } \\
\text { expectations }\end{array}$ & $\begin{array}{l}\text { Always } \\
\text { meet } \\
\text { expectations }\end{array}$ & $\begin{array}{l}\text { Expectations } \\
\text { exceeded } \\
\text { delighted } \\
\text { customers }\end{array}$ \\
\hline Po7c Employee Morale & Very low & low & satisfactory & high & very high \\
\hline Po7d Productivity & decreasing & static & $\begin{array}{l}\text { moderate } \\
\text { improvement }\end{array}$ & $\begin{array}{l}\text { Consistently } \\
\text { improving }\end{array}$ & $\begin{array}{l}\text { Major and } \\
\text { significant } \\
\text { gains }\end{array}$ \\
\hline $\begin{array}{l}\text { Po8a Defects as a } \% \\
\text { Of production volume }\end{array}$ & $<0.1 \%$ & $0.1-0.49 \%$ & $0.5-1.99 \%$ & $2.0-5.0 \%$ & $>5.0 \%$ \\
\hline $\begin{array}{l}\text { Po } 8 b \text { Warranty claims } \\
\text { Cost as a } \% \text { of total sales }\end{array}$ & $<0.1 \%$ & $0.1-0.99 \%$ & $0.5-1.49 \%$ & $1.5-3.0 \%$ & $>3.0 \%$ \\
\hline $\begin{array}{l}\text { Po8c Cost of quality } \\
\text { (error, scrap, rework and } \\
\text { inspection) as a \% of total } \\
\text { sales }\end{array}$ & $<1.0 \%$ & $1.0-4.9 \%$ & $5.0-9.9 \%$ & $10.0-15.0 \%$ & $>15.0 \%$ \\
\hline $\begin{array}{l}\text { Delivery in full on time } \\
\text { to our customer }\end{array}$ & $<50 \%$ & $50-80 \%$ & $81-90 \%$ & $91-96 \%$ & $97-100 \%$ \\
\hline
\end{tabular}


Appendix A6 - Final Measurement Instrument Used

\section{Exploring the Quality-I nnovation Connection Questionnaire}

The Oregon Wood Innovation Center is conducting a study to identify companies that are successfully achieving innovation goals through the use of quality tools and techniques. Our goal is to identify specific quality management practices that lead to improved innovation performance. We thank you in advance for your assistance with this effort.

You may return this survey in the enclosed postage-paid envelope or by fax at (541) 737-3385. If you prefer to complete the questionnaire on-line, please see http://owic.oregonstate.edu/quality/survey.php

Questions? Please contact Scott Leavengood, (541) 737-4212 or Scott.Leavengood@oregonstate.edu.

Your job title: Number of years with the organization:

\begin{tabular}{|c|c|c|c|c|}
\hline A. Leadership & $\begin{array}{l}1=\text { strongly } \\
\text { disagree }\end{array}$ & & & $\begin{array}{r}\text { strongly } \\
\text { agree }\end{array}$ \\
\hline $\begin{array}{l}\text { 1. Senior executives share similar beliefs about the future } \\
\text { direction of this organization. }\end{array}$ & 12 & 3 & 4 & 5 \\
\hline $\begin{array}{l}\text { 2. Senior managers actively encourage change and implement a } \\
\text { culture of improvement, learning, and innovation in pursuit of } \\
\text { 'excellence.' }\end{array}$ & 12 & 3 & 4 & 5 \\
\hline $\begin{array}{l}\text { 3. Employees have the opportunity to share in and are } \\
\text { encouraged to help the organization implement changes. }\end{array}$ & 12 & 3 & 4 & 5 \\
\hline $\begin{array}{l}\text { 4. There is a high degree of unity of purpose in our company, and } \\
\text { we have eliminated barriers between individuals and/or } \\
\text { departments. }\end{array}$ & 12 & J & 4 & 5 \\
\hline
\end{tabular}

\begin{tabular}{|c|c|c|c|}
\hline B. People Management & $\begin{array}{l}1 \text { = strongly } \\
\text { disagree }\end{array}$ & & $\begin{array}{r}5=\text { strongly } \\
\text { agree }\end{array}$ \\
\hline $\begin{array}{l}\text { 5. We have an organization-wide training and development } \\
\text { process, including career path planning, for all our employees }\end{array}$ & 12 & 3 & 45 \\
\hline $\begin{array}{l}\text { 6. Our company has maintained both 'top-down' and 'bottom-up' } \\
\text { communication processes. }\end{array}$ & 12 & 3 & 45 \\
\hline 7. Employee satisfaction is formally and regularly measured. & 12 & 3 & 45 \\
\hline 8. Employee flexibility, multi-skilling and training are actively & 12 & 3 & 45 \\
\hline
\end{tabular}




\begin{tabular}{|l|llllll|}
\hline used to support performance improvement. & & & & & & \\
\hline $\begin{array}{l}\text { 9. We always maintain a work environment that contributes to } \\
\text { the health, safety and well-being of all employees. }\end{array}$ & & 1 & 2 & 3 & 4 & 5 \\
\hline
\end{tabular}

\begin{tabular}{|c|c|c|c|c|}
\hline C. Customer Focus & $\begin{array}{l}1 \text { = strongly } \\
\text { disagree }\end{array}$ & & & rongly \\
\hline $\begin{array}{l}\text { 10. We actively and regularly seek customer inputs to identify } \\
\text { their needs and expectations. }\end{array}$ & 12 & 3 & 4 & 5 \\
\hline $\begin{array}{l}\text { 11. Customer needs and expectations are effectively } \\
\text { disseminated and understood throughout the workforce. }\end{array}$ & 12 & 3 & 4 & 5 \\
\hline 12. We involve customers in our product design processes. & 12 & 3 & 4 & 5 \\
\hline $\begin{array}{l}\text { 13. We always maintain a close relationship with our customers } \\
\text { and provide them an easy channel for communicating with us. }\end{array}$ & 12 & & 4 & 5 \\
\hline $\begin{array}{l}\text { 14. We have an effective process for resolving customers' } \\
\text { complaints. }\end{array}$ & 12 & 3 & 4 & 5 \\
\hline $\begin{array}{l}\text { 15. We systematically and regularly measure customer } \\
\text { satisfaction. }\end{array}$ & 12 & 3 & 4 & 5 \\
\hline D. Strategic Planning & $\begin{array}{l}1 \text { = strongly } \\
\text { disagree }\end{array}$ & & & $\begin{array}{r}\text { strongly } \\
\text { agree }\end{array}$ \\
\hline $\begin{array}{l}\text { 16. We have a mission statement which has been communicated } \\
\text { throughout the company and is supported by our employees. }\end{array}$ & 12 & & 4 & 5 \\
\hline $\begin{array}{l}\text { 17. We have a comprehensive and structured planning process } \\
\text { which regularly sets and reviews short and long-term goals. }\end{array}$ & 12 & 3 & 4 & 5 \\
\hline $\begin{array}{l}\text { 18. When we develop our plans, policies and objectives we } \\
\text { always incorporate the needs of all stakeholders, including the } \\
\text { community. }\end{array}$ & 12 & J & + & 5 \\
\hline $\begin{array}{l}\text { 19. We have a written statement of strategy covering all business } \\
\text { operations which is articulated and agreed to by our Senior } \\
\text { Managers. }\end{array}$ & 12 & $J$ & 4 & 5 \\
\hline
\end{tabular}

\section{E. Process Management}

\begin{tabular}{|l|r|}
\hline $\begin{array}{l}1=\text { strongly } \\
\text { disagree }\end{array}$ & $\begin{array}{r}5=\text { strongly } \\
\text { agree }\end{array}$ \\
\hline
\end{tabular}

20. The concept of the 'internal customer' (i.e., the next process down the line) is well understood in our company.

$\begin{array}{lllll}1 & 2 & 3 & 4 & 5\end{array}$

21. We design processes in our plant to be 'fool-proof' (preventive-oriented).

22. We have clear, standardized and documented process instructions which are well understood by our employees.

23. We make extensive use of statistical techniques (e.g., SPC) to improve the processes and to reduce variation.

24. We strive to establish long-term relationships with suppliers.

$\begin{array}{lllll}1 & 2 & 3 & 4 & 5\end{array}$

$\begin{array}{lllll}1 & 2 & 3 & 4 & 5\end{array}$

$\begin{array}{lllll}1 & 2 & 3 & 4 & 5\end{array}$

$\begin{array}{lllll}1 & 2 & 3 & 4 & 5\end{array}$


25. We use a supplier rating system to select our suppliers and monitor their performance.

F. Information \& Analysis

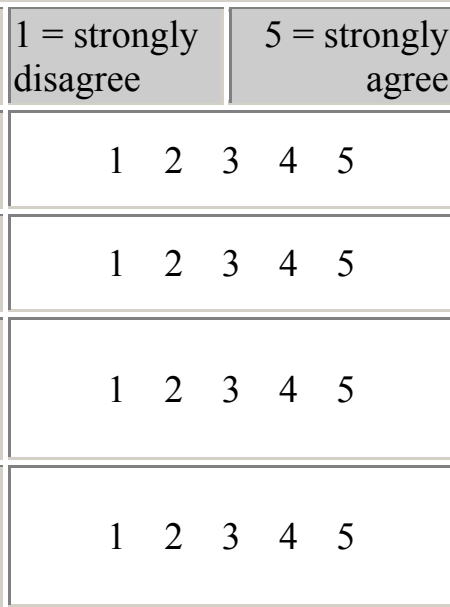

26. Our company has an effective performance measurement system to track overall organization performance.

27. Up-to-date data and information of company performance are always readily available for those who need them.

28. Senior management regularly meet to review company performance and use the information as a basis for decisionmaking.

29. We are engaged in an active competitive benchmarking program to measure our performance against the 'best practice' in the industry.

\begin{tabular}{|c|c|c|c|}
\hline G. Quality & $\begin{array}{l}1=\text { worst in } \\
\text { industry }\end{array}$ & & $\begin{array}{r}5=\text { best in } \\
\text { industry }\end{array}$ \\
\hline 30. The performance of our products. & 12 & 3 & 45 \\
\hline 31. Conformance to specifications of our products. & 12 & 3 & 45 \\
\hline 32. Reliability of our products. & 12 & 3 & 45 \\
\hline 33. Durability of our products. & 12 & 3 & 45 \\
\hline
\end{tabular}

\begin{tabular}{|l}
\hline H. Product Innovation \\
\begin{tabular}{|l} 
34. The level of newness (novelty) of our firm's new products. \\
35. The use of the latest technological innovations in our new \\
products.
\end{tabular} \\
\hline
\end{tabular}

36. The speed of our new product development.

37. The number of new products our firm has introduced to the market.

\begin{tabular}{|l|r|}
\hline $\begin{array}{l}1=\text { worst in } \\
\text { industry }\end{array}$ & $\begin{array}{r}5=\text { best in } \\
\text { industry }\end{array}$ \\
\hline
\end{tabular}

38. The number of our new products that is first-to-market (early market entrants).

\begin{tabular}{|c|c|c|c|}
\hline I. Process Innovation & $\begin{array}{l}1=\text { worst in } \\
\text { industry }\end{array}$ & & $\begin{array}{l}5=\text { best in } \\
\text { industry }\end{array}$ \\
\hline 39. The technological competitiveness of our company. & 12 & 3 & 45 \\
\hline $\begin{array}{l}\text { 40. The speed with which we adopt the latest technological } \\
\text { innovations in our processes. }\end{array}$ & 12 & 3 & 5 \\
\hline $\begin{array}{l}\text { 41. The level of newness (state-of-the-art) of the technology } \\
\text { used in our processes. }\end{array}$ & 12 & 5 & 5 \\
\hline
\end{tabular}


42. The rate of change in our processes, techniques and technology.

\begin{tabular}{|c|c|c|c|}
\hline J. Business Systems Innovation & $\begin{array}{l}1=\text { worst in } \\
\text { industry }\end{array}$ & & $\begin{array}{l}5=\text { best in } \\
\text { industry }\end{array}$ \\
\hline 43. Our ability to penetrate new markets. & 12 & 3 & 45 \\
\hline 44. Our ability to obtain new sources of supply. & 12 & 3 & 45 \\
\hline 45. Our success in seeking new ways of organizing our business. & 12 & 3 & 45 \\
\hline
\end{tabular}

Thank you for your time! 


\section{Appendix A7 - Cover Letter for Questionnaire}

Dear $<$ Salutation $><$ Last Name $>$ :

My name is Scott Leavengood and I am the director of the Oregon Wood Innovation Center at Oregon State University. I am also a PhD student at Portland State University. For my dissertation, I am beginning a study to identify best quality management practices for attaining quality and innovation performance and would like to invite your participation.

You are being asked to take part because you are a manager in a wood product manufacturing company or a firm that is a supplier to the wood products industry. I am interested in your perception of quality management practices and business performance in your company. The information I collect will help us to better understand how specific quality management practices are related to performance with respect to both quality and innovation. If you decide to participate, you will be asked to complete the enclosed survey. It should take approximately 30 minutes to complete the survey. I may also contact you to conduct an in-person interview to gain more in-depth understanding of your quality management practices.

You, and your company, may benefit from the results of this study through a better understanding of best practices in quality management that lead to improved quality and innovation performance.

Any information that is obtained in connection with this study and that can be linked to you or identify you will be kept confidential. Your identity and company name do not appear on the survey; I will record responses only by the ID number in the upper right corner of the enclosed survey. A key that links your company and the ID number will be stored in a password-protected file. Published results of the study will not list you or your company name, but will identify your company only by the ID number.

Participation in either the survey or the follow-up interviews is entirely voluntary, however you must be over 18 years old to participate. Your decision to participate or not will not affect your relationship with the researcher or with the Oregon Wood Innovation Center or Portland State University in any way. If you decide not to take part in the study, you may choose to withdraw at any time without penalty. Please keep a copy of this letter for your records.

If you have concerns or problems with your participation in this study or your rights as a research subject, please contact the Human Subjects Research Review Committee, Office of Research and Sponsored Projects, 111 Cramer Hall, Portland State University, (503) 725-4288/ 1-877-480-4400. If you have questions about the study itself, contact Scott Leavengood at the address and number in the letterhead.

Thank you for your time.

Sincerely,

Scott Leavengood

Director, Oregon Wood Innovation Center, Oregon State University

and

Ph.D. candidate, Systems Science/ Engineering and Technology Management, Portland State

University 
Appendix A8 - Case Study Interview Questions

\section{Leadership}

1. Describe a significant change your company has recently experienced - for example, a shift to serve a new market, use of e-commerce, launch of a new product line, implementation of lean manufacturing, etc.

a. What was the role of senior managers in the change process?

b. What was the role of employees?

2. In what way do senior managers work to create a culture conducive to innovation?

\section{People Management}

3. How do you assess employee satisfaction?

a. Describe the process (e.g., questionnaires, focus groups, interviews, etc.)

b. How often do you assess it?

c. How do you use the information obtained?

4. Regarding employee training \& development:

a. How are new employees trained?

b. How do you identify training needs?

c. How do you organize/offer training courses?

d. Do you practice cross-training (multi-skilling)? If yes, how is this done?

5. Describe the modes of communication in your company - How do managers

communicate to employees and vice-versa?

\section{Customer Focus}

6. How do you obtain input/feedback from customers?

a. How is the input communicated within the company?

7. How do you involve customers in the design process?

8. How do you measure customer satisfaction?

a. Describe the process - who is responsible for collecting the information?

How is it done - questionnaires, interviews, etc?

b. How often is it measured?

c. How do you use the information?

\section{Strategic Planning}

9. Do you have mission and vision statements? If so, how are these communicated and to whom?

10. What sort of high-level planning do you do?

a. Who is involved?

b. How often is it done?

c. Do you compare results to plan?

\section{Process Management}

11. Is the concept of the 'internal customer' (next process down the line) something you emphasize?
a. If so, how? 
12. Which of the following 'quality tools' do you use?:

Pareto analysis, Statistical process control (SPC), Checksheets, Cause-andeffect diagrams, Histograms, Design of experiments (or other statistical tools like ANOVA), Simulation, Operations research (linear programming), Flowcharting or value stream mapping, Kaizen events, Others?

13. In what areas of the company are these tools used? (e.g., sales, manufacturing, administration, etc.)

\section{Information \& Analysis}

14. What are your key measures for assessing overall company performance?

a. How and to whom are these communicated?

15. Do you benchmark other companies (either in your industry or another industry)? If so,

a. Describe one example of how you did this and the results.

\section{Quality}

16. How do you define quality for your product?

a. How do you measure quality?

\section{Product Innovation}

17. How do you conduct research and development (R\&D)?

18. List some examples of new products you've developed in the last 3-5 years.

19. What types of technological innovations have you developed or adopted related to your products in the last 3-5 years (for example, new fasteners, finishes, adhesives, etc.)

a. Do you look to such innovations for competitive advantage? (or are they simply required for the industry)

20. [Scenario] - One of your customers presents you with an idea for a new product. How would you go about evaluating the opportunity?

a. What are the barriers to developing new products more rapidly?

\section{Process Innovation}

21. With respect to the technology/machinery used to produce your product:

a. How would you describe the rate of change in the technology in your industry - slow, stable, or rapidly changing?

b. Would you describe the technology you use as "state-of-the art" (among the latest technology available) or "perhaps not the latest, but still efficient and effective?"

c. How would you compare your company with competitors (with respect to processing technology)?

d. Do you actively investigate new technologies as they become available?

(e.g., computerized routers, scanning systems/optimizers, robotics/automated assembly systems, etc.) Why or why not? What is the role of equipment vendors in this process? 


\section{Business Systems Innovation}

22. What about new or innovative ways for managing your business:

a. Do you have any examples of new ways you have developed or adopted to manage your business?

b. Has the way you market your products changed in a significant way in the past 3-5 years? How about the way you distribute/deliver products?

c. Do you have a company website? If so, do you use it to sell products (ecommerce)? Do you use it as a way to obtain input from customers \& suppliers?

d. Have you sought new sources of supply recently? If so, what prompted you to do so? How did you find the new supplier? 
Appendix B - Innovation Constructs and Measurement Instruments 
Appendix B1- Johannessen et al.

Questions used for measurement of innovation [76]

Has your company made changes during the last three years that were perceived to be new for the company, within the following areas? (Please circle one number in each row)

\begin{tabular}{lccccc}
\hline & To no extent & $\begin{array}{c}\text { To a little } \\
\text { extent }\end{array}$ & $\begin{array}{c}\text { To some } \\
\text { extent }\end{array}$ & $\begin{array}{c}\text { To a great } \\
\text { extent }\end{array}$ & $\begin{array}{c}\text { To a very } \\
\text { great extent }\end{array}$ \\
\hline $\begin{array}{l}\text { New products } \\
\text { New services }\end{array}$ & 1 & 2 & 3 & 4 & 5 \\
$\begin{array}{l}\text { New methods } \\
\text { of production }\end{array}$ & 1 & 2 & 3 & 4 & 5 \\
$\begin{array}{l}\text { Opening new } \\
\text { markets }\end{array}$ & 1 & 2 & 3 & 4 & 5 \\
$\begin{array}{l}\text { New sources } \\
\text { of supply }\end{array}$ & 1 & 2 & 3 & 4 & 5 \\
$\begin{array}{l}\text { New ways of } \\
\text { organizing }\end{array}$ & 1 & 2 & 3 & 4 & 5 \\
\hline
\end{tabular}

Note: The instrument could be repeated with 'new to the industry' (vs. company) to address the issue of radical vs. incremental forms of innovation. 
Appendix C - Graphical Representation of Efficiency

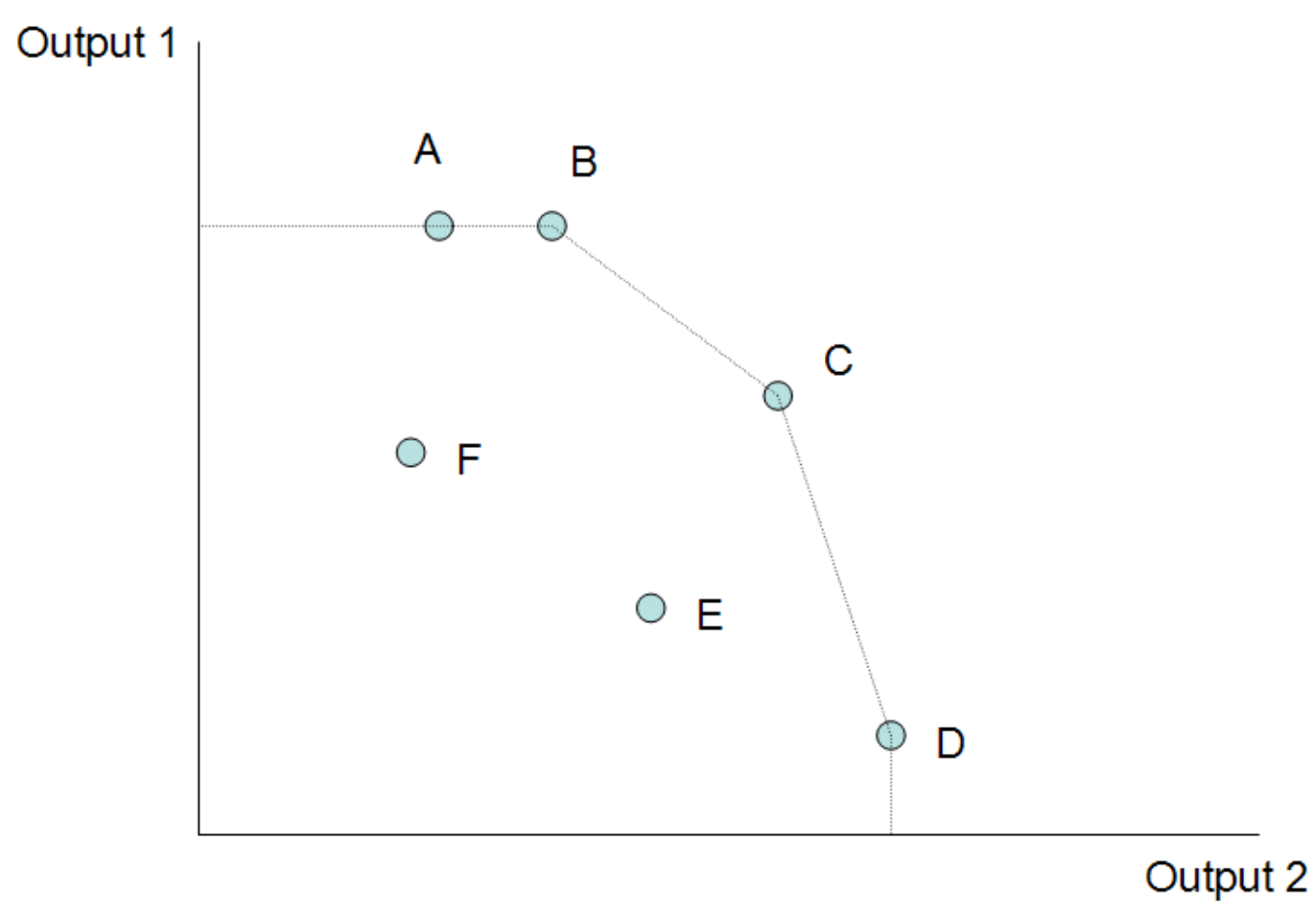

DMUs A, B, C, and D are all efficient. DMUs E and F are inefficient. However, DMU A is weakly efficient compared to B because A could increase its output of Output 2 while maintaining the same level of Output 1. 
Appendix D - Results of Test for Non-response Bias

Tested first 30 responses vs. last 30 responses for all questions in each category

Leadership

\begin{tabular}{|l|l|l|l|}
\hline Question & Mean (sd) & Std. Error mean & Sig. (2-tailed) \\
\hline A1 - first 30 & $4.03(1.129)$ & 0.206 & 0.710 \\
A1 - last 30 & $4.13(0.937)$ & 0.171 & \\
\hline A2 - first 30 & $4.07(0.868)$ & 0.159 & 0.807 \\
A2 - last 30 & $4.00(1.203)$ & 0.220 & \\
\hline A3 - first 30 & $3.83(1.117)$ & 0.204 & 0.899 \\
A3 - last 30 & $3.87(0.900)$ & 0.164 & \\
\hline A4- first 30 & $3.67(0.884)$ & 0.161 & 1.00 \\
A4 - last 30 & $3.67(1.093)$ & 0.200 & \\
\hline
\end{tabular}

People Management

\begin{tabular}{|l|l|l|l|}
\hline Question & Mean (sd) & Std. Error mean & Sig. (2-tailed) \\
\hline B5 - first 30 & $2.66(0.974)$ & 0.181 & 0.598 \\
B5 - last 30 & $2.50(1.253)$ & 0.229 & \\
\hline B6 - first 30 & $3.83(1.020)$ & 0.186 & 0.126 \\
B6 - last 30 & $3.41(1.053)$ & 0.195 & \\
\hline B7 - first 30 & $2.77(1.305)$ & 0.238 & 0.698 \\
B7 - last 30 & $2.90(1.348)$ & 0.246 & \\
\hline B8 - first 30 & $3.47(1.137)$ & 0.208 & 0.392 \\
B8 - last 30 & $3.70(0.952)$ & 0.174 & \\
\hline B9 - first 30 & $4.13(1.106)$ & 0.202 & 0.727 \\
B9 - last 30 & $4.23(1.104)$ & 0.202 & \\
\hline
\end{tabular}

\section{Customer Focus}

\begin{tabular}{|l|l|l|l|}
\hline Question & Mean $(\mathrm{sd})$ & Std. Error mean & Sig. (2-tailed) \\
\hline C10 - first 30 & $3.83(0.950)$ & 0.173 & 0.889 \\
C10 - last 30 & $3.87(0.900)$ & 0.164 & \\
\hline C11 - first 30 & $3.70(0.837)$ & 0.153 & 0.761 \\
C11 - last 30 & $3.63(0.850)$ & 0.155 & \\
\hline C12 - first 30 & $3.55(1.183)$ & 0.220 & 0.779 \\
C12 - last 30 & $3.63(1.033)$ & 0.189 & \\
\hline C13 - first 30 & $4.27(0.785)$ & 0.143 & 0.171 \\
C13 - last 30 & $3.97(0.890)$ & 0.162 & \\
\hline C14 - first 30 & $4.00(1.050)$ & 0.192 & 0.893 \\
C14 - last 30 & $3.97(0.850)$ & 0.155 & \\
\hline C15 - first 30 & $2.90(1.242)$ & 0.227 & 0.526 \\
C15 - last 30 & $3.10(1.205)$ & 0.224 & \\
\hline
\end{tabular}


Strategic Planning

\begin{tabular}{|l|l|l|l|}
\hline Question & Mean (sd) & Std. Error mean & Sig. (2-tailed) \\
\hline D16 - first 30 & $3.03(1.377)$ & 0.251 & 0.923 \\
D16 - last 30 & $3.07(1.285)$ & 0.235 & \\
\hline D17 - first 30 & $3.10(1.155)$ & 0.211 & 0.418 \\
D17 - last 30 & $2.83(1.367)$ & 0.250 & \\
\hline D18 - first 30 & $3.03(1.017)$ & 0.189 & 0.249 \\
D18 - last 30 & $2.70(1.179)$ & 0.215 & \\
\hline D19 - first 30 & $2.76(1.154)$ & 0.214 & 0.105 \\
D19 - last 30 & $2.27(1.143)$ & 0.209 & \\
\hline
\end{tabular}

\section{Process Management}

\begin{tabular}{|l|l|l|l|}
\hline Question & Mean (sd) & Std. Error mean & Sig. (2-tailed) \\
\hline E20 - first 30 & $3.17(1.234)$ & 0.225 & 0.918 \\
E20 - last 30 & $3.20(1.270)$ & 0.232 & \\
\hline E21 - first 30 & $3.52(0.911)$ & 0.169 & 0.086 \\
E21 - last 30 & $3.10(0.923)$ & 0.168 & \\
\hline E22 - first 30 & $3.40(1.070)$ & 0.195 & 0.149 \\
E22 - last 30 & $3.00(1.050)$ & 0.192 & \\
\hline E23 - first 30 & $2.37(1.098)$ & 0.200 & 0.307 \\
E23 - last 30 & $2.67(1.155)$ & 0.211 & \\
\hline E24 - first 30 & $4.33(0.959)$ & 0.175 & 0.885 \\
E24 - last 30 & $4.37(0.809)$ & 0.148 & \\
\hline E25 - first 30 & $2.70(1.393)$ & 0.254 & 0.685 \\
E25 - last 30 & $2.57(1.223)$ & 0.223 & \\
\hline
\end{tabular}

Information \& Analysis

\begin{tabular}{|l|l|l|l|}
\hline Question & Mean $(\mathrm{sd})$ & Std. Error mean & Sig. (2-tailed) \\
\hline F26 - first 30 & $2.90(1.235)$ & 0.229 & 0.313 \\
F26 - last 30 & $3.23(1.305)$ & 0.238 & \\
\hline F27 - first 30 & $3.23(1.135)$ & 0.207 & 0.840 \\
F27 - last 30 & $3.30(1.393)$ & 0.254 & \\
\hline F28 - first 30 & $3.43(1.194)$ & 0.218 & 0.916 \\
F28 - last 30 & $3.40(1.248)$ & 0.228 & \\
\hline F29 - first 30 & $2.79(1.177)$ & 0.218 & 0.723 \\
F29 - last 30 & $2.09(1.125)$ & 0.205 & \\
\hline
\end{tabular}

\section{Quality}

\begin{tabular}{|l|l|l|l|}
\hline Question & Mean (sd) & Std. Error mean & Sig. (2-tailed) \\
\hline G30 - first 30 & $4.47(0.681)$ & 0.124 & 1.000 \\
G30 - last 30 & $4.47(0.507)$ & 0.093 & \\
\hline G31 - first 30 & $4.55(0.506)$ & 0.094 & 0.154 \\
G31 - last 30 & $4.30(0.794)$ & 0.145 & \\
\hline G32 - first 30 & $4.43(0.568)$ & 0.104 & 0.920 \\
G32 - last 30 & $4.45(0.572)$ & 0.106 & \\
\hline G33 - first 30 & $4.53(0.629)$ & 0.115 & 0.487 \\
G33 - last 30 & $4.41(0.682)$ & 0.127 & \\
\hline
\end{tabular}


Product Innovation

\begin{tabular}{|l|l|l|l|}
\hline Question & Mean (sd) & Std. Error mean & Sig. (2-tailed) \\
\hline H34 - first 30 & $3.59(1.018)$ & 0.189 & 0.652 \\
H34 - last 30 & $3.48(0.688)$ & 0.128 & \\
\hline H35 - first 30 & $3.27(1.202)$ & 0.219 & 0.441 \\
H35 - last 30 & $3.48(0.911)$ & 0.169 & \\
\hline H36 - first 30 & $3.10(0.976)$ & 0.181 & 0.233 \\
H36 - last 30 & $3.41(0.983)$ & 0.182 & \\
\hline H37 - first 30 & $3.21(0.861)$ & 0.160 & 0.648 \\
H37 - last 30 & $3.32(1.020)$ & 0.193 & \\
\hline H38 - first 30 & $2.96(0.980)$ & 0.189 & 0.993 \\
H38 - last 30 & $2.97(1.052)$ & 0.195 & \\
\hline
\end{tabular}

Process Innovation

\begin{tabular}{|l|l|l|l|}
\hline Question & Mean (sd) & Std. Error mean & Sig. (2-tailed) \\
\hline I39 - first 30 & $3.30(1.055)$ & 0.193 & 0.392 \\
I39 - last 30 & $3.53(1.042)$ & 0.190 & \\
\hline I40 - first 30 & $3.00(1.017)$ & 0.186 & 0.807 \\
I40 - last 30 & $3.07(1.081)$ & 0.197 & \\
\hline I41 - first 30 & $3.00(1.017)$ & 0.186 & 0.560 \\
I41 - last 30 & $3.17(1.177)$ & 0.215 & \\
\hline I42 - first 30 & $3.13(0.860)$ & 0.157 & 0.480 \\
I42 - last 30 & $3.30(0.952)$ & 0.174 & \\
\hline
\end{tabular}

Business Systems Innovation

\begin{tabular}{|l|l|l|l|}
\hline Question & Mean (sd) & Std. Error mean & Sig. (2-tailed) \\
\hline J43 - first 30 & $3.10(0.759)$ & 0.139 & 0.733 \\
J43 - last 30 & $3.17(0.747)$ & 0.136 & \\
\hline J44 - first 30 & $4.00(0.643)$ & 0.117 & $0.014^{*}$ \\
J44 - last 30 & $3.43(1.040)$ & 0.190 & \\
\hline J45 - first 30 & $3.43(0.774)$ & 0.141 & 0.371 \\
J45 - last 30 & $3.23(0.935)$ & 0.171 & \\
\hline
\end{tabular}

*J44 (Ability to obtain new sources of supply) - non-respondents may be lower on this point 
Appendix E - DEA Results

\begin{tabular}{|c|c|c|c|c|c|c|c|c|c|c|c|c|}
\hline DMU & 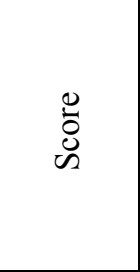 & 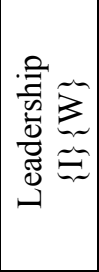 & 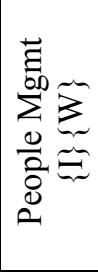 & 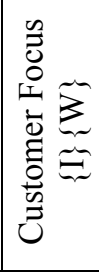 & 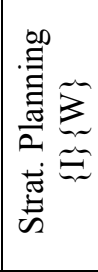 & 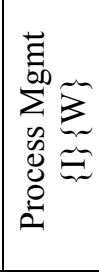 & 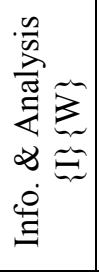 & 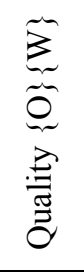 & 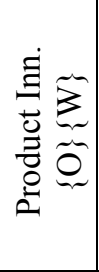 & 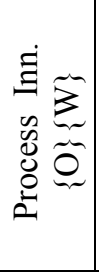 & 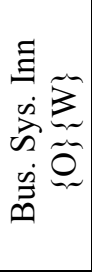 & 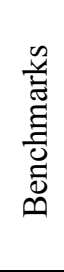 \\
\hline D2740 & $46.05 \%$ & 0.46 & 0 & 0 & 0 & 0 & 0 & 0 & 0.07 & 0.14 & 0 & 151 \\
\hline D712 & $63.49 \%$ & 0 & 0.63 & 0 & 0 & 0 & 0 & 0.16 & 0 & 0 & 0.1 & 30 \\
\hline D526 & $65.60 \%$ & 0.16 & 0 & 0 & 0.38 & 0 & 0 & 0 & 0.15 & 0.04 & 0.1 & 67 \\
\hline D1485 & $66.40 \%$ & 0 & 0 & 0 & 0 & 0.66 & 0 & 0.21 & 0 & 0.06 & 0 & 22 \\
\hline D143 & $69.89 \%$ & 0.08 & 0 & 0 & 0 & 0.32 & 0.1 & 0 & 0 & 0 & 0.3 & 98 \\
\hline D1508 & $75.49 \%$ & 0 & 0 & 0.37 & 0.39 & 0 & 0 & 0 & 0.38 & 0 & 0 & 8 \\
\hline D947 & $75.88 \%$ & 0 & 0 & 0 & 0.18 & 0.38 & 0 & 0.09 & 0.2 & 0 & 0 & 90 \\
\hline D519 & $77.25 \%$ & 0 & 0 & 0 & 0.16 & 0 & 0.57 & 0 & 0.13 & 0.02 & 0.18 & 3 \\
\hline D1467 & $79.70 \%$ & 0 & 0 & 0 & 0.12 & 0.22 & 0.19 & 0.2 & 0 & 0.03 & 0 & 47 \\
\hline D305 & $82.95 \%$ & 0 & 0 & 0.08 & 0.18 & 0.15 & 0 & 0 & 0 & 0.2 & 0 & 58 \\
\hline D1006 & $83.36 \%$ & 0 & 0 & 0 & 0.26 & 0 & 0.29 & 0 & 0 & 0.16 & 0.04 & 4 \\
\hline D1833 & $84.64 \%$ & 0 & 0 & 0 & 0.21 & 0.12 & 0.09 & 0 & 0.13 & 0.07 & 0 & 17 \\
\hline D1261 & $85.80 \%$ & 0 & 0.31 & 0.17 & 0.15 & 0 & 0 & 0 & 0 & 0 & 0.33 & 20 \\
\hline D1235 & $86.44 \%$ & 0 & 0 & 0 & 0.05 & 0.24 & 0.11 & 0 & 0.06 & 0.07 & 0.1 & 62 \\
\hline D1343 & $87.72 \%$ & 0 & 0 & 0 & 0.88 & 0 & 0 & 0.14 & 0.09 & 0 & 0 & 1 \\
\hline D385 & $88.04 \%$ & 0 & 0 & 0.01 & 0.53 & 0.01 & 0.17 & 0.17 & 0 & 0 & 0.04 & 16 \\
\hline D2113 & $90.97 \%$ & 0 & 0.13 & 0 & 0.12 & 0.25 & 0 & 0.05 & 0.05 & 0.05 & 0.14 & 84 \\
\hline D243 & $91.56 \%$ & 0 & 0 & 0 & 0 & 0.39 & 0.11 & 0.12 & 0.08 & 0.02 & 0.02 & 1 \\
\hline D1543 & $92.42 \%$ & 0.17 & 0 & 0 & 0.22 & 0.34 & 0 & 0.31 & 0.03 & 0 & 0 & 10 \\
\hline D1431 & $92.71 \%$ & 0 & 0.08 & 0 & 0 & 0.35 & 0 & 0 & 0.11 & 0 & 0.15 & 46 \\
\hline D471 & $93.10 \%$ & 0 & 0.27 & 0 & \begin{tabular}{|l|}
0.17 \\
\end{tabular} & 0 & 0.02 & 0 & 0 & 0 & 0.21 & 2 \\
\hline D958 & $93.61 \%$ & 0 & 0.15 & 0 & 0 & 0 & 0.44 & 0.16 & 0.05 & 0 & 0 & 1 \\
\hline D54 & $96.32 \%$ & 0 & 0 & 0.04 & 0.05 & 0.31 & 0.04 & 0.12 & 0.04 & 0.07 & 0 & 8 \\
\hline D940 & $98.00 \%$ & 0 & 0 & 0 & 0.25 & 0.31 & 0 & 0.01 & 0.07 & 0 & 0.22 & 0 \\
\hline D2967 & $98.29 \%$ & 0 & 0 & 0 & 0.54 & 0.18 & 0 & 0.18 & 0.02 & 0.03 & 0 & 2 \\
\hline D758 & $98.43 \%$ & 0 & 0 & 0 & \begin{tabular}{|l|}
0.98 \\
\end{tabular} & 0 & 0 & 0 & 0.05 & 0.22 & 0 & 0 \\
\hline
\end{tabular}

Note:

DMU: "Decision making unit", i.e., individual companies

Score: Output-oriented super-efficiency score; values of $100 \%$ or less are considered efficient. $(\mathrm{I})(\mathrm{W})$ : optimal weighted inputs (vx) from Equations 1 and 2.

$(\mathrm{O})(\mathrm{W})$ : optimal weighted outputs ( $\mu \mathrm{y})$ from Equations 1 and 2.

Benchmarks: number of times this company was used in setting performance targets for the other firms in the DEA set. 